\title{
$\mu$-CONSTANT MONODROMY GROUPS AND TORELLI RESULTS FOR THE QUADRANGLE SINGULARITIES AND THE BIMODAL SERIES
}

\author{
FALKO GAUSS AND CLAUS HERTLING \\ To the memory of Egbert Brieskorn
}

\begin{abstract}
This paper is a sequel to [He11] and GH17. In [He11] a notion of marking of isolated hypersurface singularities was defined, and a moduli space $M_{\mu}^{\text {mar }}$ for marked singularities in one $\mu$-homotopy class of isolated hypersurface singularities was established. It is an analogue of a Teichmüller space. It comes together with a $\mu$-constant monodromy group $G^{\text {mar }} \subset G_{\mathbb{Z}}$. Here $G_{\mathbb{Z}}$ is the group of automorphisms of a Milnor lattice which respect the Seifert form. It was conjectured that $M_{\mu}^{m a r}$ is connected. This is equivalent to $G^{\text {mar }}=G_{\mathbb{Z}}$. Also Torelli type conjectures were formulated. In He11] and [GH17] $M_{\mu}^{\text {mar }}, G_{\mathbb{Z}}$ and $G^{\text {mar }}$ were determined and all conjectures were proved for the simple, the unimodal and the exceptional bimodal singularities. In this paper the quadrangle singularities and the bimodal series are treated. The Torelli type conjectures are true. But the conjecture $G^{\text {mar }}=G_{\mathbb{Z}}$ and $M_{\mu}^{m a r}$ connected does not hold for certain subseries of the bimodal series.
\end{abstract}

\section{Contents}

1. Introduction

2. $\mathbb{Z}$-lattices with unimodal bilinear form and monodromy 9

3. Some Fuchsian groups 18

4. Review on the topology of singularities [26]

5. The group $G_{\mathbb{Z}}$ for the bimodal series singularities 30

6. The group $G_{\mathbb{Z}}$ for the quadrangle singularities 66

7. Gauss-Manin connection and Brieskorn lattice 71

Date: October 10, 2017.

2000 Mathematics Subject Classification. 32S15, 32S40, 14D22, 58K70.

Key words and phrases. $\mu$-constant monodromy group, marked singularity, moduli space, Torelli type problem, quadrangle singularities, bimodal series.

This work was supported by the DFG grant He2287/4-1 (SISYPH). Moreover the first author gratefully acknowledges support from the JSPS Postdoctoral Fellowship for Research in Japan ID No. PE17702. 
8. Review on marked singularities, their moduli spaces, $\mu$-constant monodromy groups and Torelli conjectures

9. Period maps and Torelli results for the bimodal series and $G_{\mathbb{Z}} \supsetneqq G^{\text {mar }}$ for the subseries

10. Period maps and Torelli results for the quadrangle singularities

References

\section{INTRODUCTION}

We dedicate this paper to the memory of Egbert Brieskorn. It has its roots in work which the second author, Claus Hertling, had done as a student of Brieskorn in Bonn in the early 90'ies.

1.1. Reminiscences of the second author. Prof. Dr. Egbert Brieskorn accepted me as a diploma student in the spring of 1989 . On March 10 and 13, 1989, he gave two full days (Friday + Monday) of lectures for his new diploma students (including me) and doctoral students. I still have his handwritten manuscript of 52 pages. There he introduced us to isolated hypersurface singularities. He talked about the Jacobi algebra, the universal unfolding with its discriminant, the Milnor fibration, its monodromy, local systems and integrable connections and systems of regular singular linear differential equations in general, his own work on the Gauss-Manin connection and especially the Brieskorn lattice, and the mixed Hodge structure which it induces. He strongly recommended to read [AGV88, SSaM89] and SS85. He proposed to me to work on the moduli of singularities using the GaussManin connection.

I followed his advice in my diploma thesis and my doctoral thesis and beyond the doctoral thesis. The subject developed into a long-going project of mine, which I took up again and again. The present paper is in some sense a final step of it.

In the doctoral thesis [He93, I formulated the global Torelli type conjecture that an isolated hypersurface singularity is determined up to right equivalence by its Brieskorn lattice together with the Milnor lattice and the Seifert form (conjecture 1.1 (b) reformulates this conjecture). I proved it in the doctoral thesis for all unimodal singularities, the exceptional bimodal singularities, the bimodal quadrangle singularities, and the bimodal series $E_{3, p}$.

For the other seven bimodal series, I made in the spring 1993, some months after finishing the doctoral thesis, long calculations (120 pages) 
which led to a proof of this Torelli type conjecture for all series except the three bimodal subseries $S_{10, r}^{\sharp}, S_{1,10 r}, Z_{1,14 r}$. At that time I thought that I would never review and publish these results. The paper He95] recapitulated the main results of the doctoral thesis and of these calculations for the eight bimodal series, but it did not at all give all details (only 2.5 pages are devoted to the bimodal series).

Later I constructed a classifying space $D_{B L}$ for Brieskorn lattices [He99] and a moduli space $M_{\mu}\left(f_{0}\right)$ of the right equivalence classes of all singularities in the $\mu$-homotopy class of a reference singularity $f_{0}$ [He02]. More recently, in [He11, I defined the notion of a marked singularity, I constructed a classifying space $M_{\mu}^{\text {mar }}\left(f_{0}\right)$ for marked singularities, and I formulated a Torelli type conjecture for marked singualarities, which is stronger than the Torelli type conjecture in the doctoral thesis for unmarked singularities.

The three papers [He11, GH17 and the present paper prove the Torelli conjecture for marked singularities for all singularities with modality 0,1 and 2. The present paper deals with the bimodal quadrangle singularities and the eight bimodal series. It comprises the calculations from the spring 1993 and adds a lot more arguments and calculations, which are necessary for the marked version.

It is satisfying, that the Torelli type conjectures hold for all singularities with modality 0,1 and 2. For each family, the interplay between the variations of the Brieskorn lattices and the automorphism group of the Milnor lattice with Seifert form is fascinating and takes the best possible shape. I believe that Brieskorn would have liked these positive results and the many techniques used for their proofs. I thank him for proposing to me in March 1989 to work on the moduli of singularities using the Gauss-Manin connection. It was a good advice.

1.2. Notions, conjectures and results. In this paper, a singularity is a holomorphic function germ $f:\left(\mathbb{C}^{n+1} \rightarrow(\mathbb{C}, 0)\right.$ with an isolated singularity at 0 . Then its Milnor lattice $M l(f) \cong \mathbb{Z}^{\mu}$ is the $\mathbb{Z}$-lattice $H_{n}\left(f^{-1}(\tau), \mathbb{Z}\right)$ for some small $\tau \in \mathbb{R}_{>0}$ for a suitable representative of $f$. Its Seifert form is called $L: M l(f) \times M l(f) \rightarrow \mathbb{Z}$. Its monodromy is called $M_{h}: M l(f) \rightarrow M l(f)$. The automorphism group of the Milnor lattice with the Seifert form is $G_{\mathbb{Z}}(f):=\operatorname{Aut}(M l(f), L)$. It will play a predominant role in this paper.

This paper is a sequel to [He11] and GH17]. In [He11, a strongly marked singularity $(f, \rho)$ and a marked singularity $(f, \pm \rho)$ are defined. Here one has to fix first a reference singularity $f_{0}$. Then $f$ is in the $\mu$-homotopy class of $f_{0}$, i.e. a $\mu$-constant family of singularities exists which contains $f_{0}$ and $f$. And $\rho:(M l(f), L(f)) \rightarrow\left(M l\left(f_{0}\right), L\left(f_{0}\right)\right)$ is 
a chosen isomorphism. Two singularities $f_{1}$ and $f_{2}$ are right equivalent if a coordinate change $\varphi$ with $f_{1}=f_{2} \circ \varphi$ exists. Two strongly marked singularities $\left(f_{1}, \rho_{1}\right)$ and $\left(f_{2}, \rho_{2}\right)$ are right equivalent if a coordinate change $\varphi$ with $f_{1}=f_{2} \circ \varphi$ and $\rho_{1}=\rho_{2} \circ(\varphi)_{\text {hom }}$ exists, where $(\varphi)_{\text {hom }}$ : $M l\left(f_{1}\right) \rightarrow M l\left(f_{2}\right)$ is the induced isomorphism.

In [He02 a moduli space $M_{\mu}\left(f_{0}\right)$ for the right equivalence classes of all singularities in the $\mu$-homotopy class of a reference singularity $f_{0}$ was constructed as an analytic geometric quotient. In [He11], this construction was enhanced to the construction of moduli spaces $M_{\mu}^{\text {mar }}\left(f_{0}\right)$ and $M_{\mu}^{\text {smar }}\left(f_{0}\right)$ of marked and strongly marked singularities. Here $M_{\mu}^{\text {smar }}\left(f_{0}\right)$ is Hausdorff and an analytic space only if assumption 8.1) or assumption 8.2 holds.

\author{
Assumption (8.1): $\quad$ Any singularity in the $\mu$-homotopy \\ class of $f_{0}$ has multiplicity $\geq 3$. \\ Assumption (8.2): Any singularity in the $\mu$-homotopy \\ class of $f_{0}$ has multiplicity 2 .
}

We expect that one of them holds for any $\mu$-homotopy class of singularities. This would be an implication of the Zariski multiplicity conjecture. But that is not proved in general.

But $M_{\mu}^{\text {mar }}\left(f_{0}\right)$ is fine, independently of these assumptions. Locally it is isomorphic to the $\mu$-constant stratum $S_{\mu}(f)$ of a singularity in the base space of a universal unfolding of that singularity. The group $G_{\mathbb{Z}}\left(f_{0}\right)$ acts properly discontinuously on $M_{\mu}^{\text {mar }}\left(f_{0}\right)$. The quotient is $M_{\mu}^{\text {mar }}\left(f_{0}\right) / G_{\mathbb{Z}} \cong M_{\mu}\left(f_{0}\right)$. Therefore that space is locally isomorphic to the quotient of $S_{\mu}(f)$ by a finite group. $M_{\mu}^{\text {mar }}\left(f_{0}\right)$ can be considered as a Teichmüller space for singularities, in analogy to the Teichmüller spaces for closed complex curves. It can also be considered as a global $\mu$-constant stratum, simultaneously for all singularities in one $\mu$-homotopy class.

The papers [He11], GH17] and this paper determine $M_{\mu}^{\operatorname{mar}}\left(f_{0}\right)$ for all singularities with modality 0,1 and 2 . The second column of the following table (1.1) gives their isomorphism classes. 


\begin{tabular}{lll} 
Singularity family & $M_{\mu}^{\text {mar }}\left(f_{0}\right)$ & $D_{B L}\left(f_{0}\right)$ \\
\hline ADE-singularities & point & point \\
simple elliptic sing. & $\mathbb{H}$ & $\mathbb{H}$ \\
hyperbolic sing. & $\mathbb{C}$ & $\mathbb{C}$ \\
exc. unimodal sing. & $\mathbb{C}$ & $\mathbb{C}$ \\
exc. bimodal sing. & $\mathbb{C}^{2}$ & $\mathbb{C}^{2}$ \\
quadrangle sing. & $(\mathbb{H}-($ a discrete set) $\times \mathbb{C}$ & $\mathbb{H} \times \mathbb{C}$ \\
the 8 series, for $m \not p$ & $\mathbb{C} * \times \mathbb{C}$ & $\mathbb{C} N_{B L}$ \\
the 8 subseries with $m \mid p$ & $\infty$ many copies of $\mathbb{C}^{*} \times \mathbb{C} \mathbb{H} \times \mathbb{C}^{N_{B L}}$
\end{tabular}

Here the eight series and the respective numbers $m$ are given in the following table $(1.2)$. Here $p \in \mathbb{Z}_{\geq 1}$.

$$
\begin{array}{lllllllll}
\text { series } & W_{1, p}^{\sharp} & S_{1, p}^{\sharp} & U_{1, p} & E_{3, p} & Z_{1, p} & Q_{2, p} & W_{1, p} & S_{1, p} \\
m & 12 & 10 & 9 & 18 & 14 & 12 & 12 & 10
\end{array}
$$

One sees that $M_{\mu}^{\text {mar }}\left(f_{0}\right)$ is simply connected for all singularities with modality 0 and 1 and for the exceptional bimodal singularities. For the quadrangle singularities and the series with $m \not p$, it is connected, but not simply connected. And for the subseries with $m \mid p$, it is not even connected, but has infinitely many components. This last result is a counterexample to conjecture 3.2 (a) in [He11, which said that $M_{\mu}^{\text {mar }}\left(f_{0}\right)$ should be connected.

In [He11], also two subgroups $G^{\text {smar }}\left(f_{0}\right)$ and $G^{\text {mar }}\left(f_{0}\right)$ of $G_{\mathbb{Z}}\left(f_{0}\right)$ were defined. $G^{\text {smar }}\left(f_{0}\right)$ was defined as the subgroup which is generated by the transversal monodromies of all $\mu$-constant families which contain $f_{0}$. Then $G^{\text {mar }}\left(f_{0}\right)$ is the group generated by $G^{\text {smar }}\left(f_{0}\right)$ and - id. A rough way to talk about this description is to say that the elements of $G^{\text {smar }}\left(f_{0}\right)$ are of geometric origin. $G^{\text {mar }}\left(f_{0}\right)$ can also be characterized as the subgroup of $G_{\mathbb{Z}}$ which maps the component $\left(M_{\mu}^{\text {mar }}\right)^{0}$ of $M_{\mu}^{\text {mar }}\left(f_{0}\right)$, which contains $\left[\left(f_{0}, \pm \mathrm{id}\right)\right]$, to itself. This last characterization gives

$$
G_{\mathbb{Z}}\left(f_{0}\right) / G^{\text {mar }}\left(f_{0}\right) \stackrel{1: 1}{\longleftrightarrow}\left\{\text { components of } M_{\mu}^{\text {mar }}\left(f_{0}\right)\right\} \text {. }
$$

In view of this, $M_{\mu}^{\text {mar }}\left(f_{0}\right)$ is connected if and only if $G_{\mathbb{Z}}\left(f_{0}\right)=G^{\text {mar }}\left(f_{0}\right)$. By table (1.1), this holds for all singularities with modality 0,1 or 2 except the eight subseries with $m \mid p$. Obviously, it is important to control $G_{\mathbb{Z}}\left(f_{0}\right)$. This was the major task in [He11 and GH17 for the singularities considered there, and it takes approximately half of this paper for the singularities considered here, the bimodal series and the quadrangle singularities. The rough outcome in all cases is that the pair $\left(M l\left(f_{0}\right), L\right)$ is surprisingly rigid and that $G_{\mathbb{Z}}\left(f_{0}\right)$ is surprisingly small. The next table (1.4) gives more information on $G_{\mathbb{Z}}\left(f_{0}\right)$ for all 
singularities with modality 0,1 and 2 . Here $M_{h} \in G_{\mathbb{Z}}$ is the classical monodromy. It commutes with all elements of $G_{\mathbb{Z}}$. The only families in table (1.4) where $\left\{ \pm M_{h}^{k} \mid k \in \mathbb{Z}\right\}$ is not finite, are the hyperbolic singularities $T_{p q r}$.

\begin{tabular}{ll} 
Singularity family & $G_{\mathbb{Z}}\left(f_{0}\right) /\left\{ \pm M_{h}^{k} \mid k \in \mathbb{Z}\right\}$ \\
\hline ADE-singularities & id $\}$ or $S_{2}$ or $S_{3}$ \\
simple elliptic sing. & a finite extension of $S L(2, \mathbb{Z})$ \\
hyperbolic sing. & a finite group \\
exc. unimodal sing. & \{id $\}$ or $S_{2}$ or $S_{3}$ \\
exc. bimodal sing. & id $\}$ or $S_{2}$ or $S_{3}$ \\
quadrangle sing. & a triangle group \\
the 8 series, for $m \backslash p$ & a cyclic finite group \\
the 8 subseries with $m \mid p$ & an infinite Fuchsian group
\end{tabular}

In the case of the eight subseries with $m \mid p, G^{\text {mar }}\left(f_{0}\right)$ is the finite subgroup of the infinite group $G_{\mathbb{Z}}\left(f_{0}\right)$ such that $G^{\text {mar }}\left(f_{0}\right) /\left\{ \pm M_{h}^{k} \mid k \in\right.$ $\mathbb{Z}\}$ is the finite cyclic group which is generated by one elliptic element.

If the $\mu$-homotopy class of $f_{0}$ contains at least one singularity with multiplicity two, then - id $\in G^{\text {smar }}\left(f_{0}\right)$ and $G^{\text {smar }}\left(f_{0}\right)=G^{\text {mar }}\left(f_{0}\right)$. Conjecture 3.2 (b) in [He11] complements this. It claims that - id $\notin$ $G^{\text {smar }}\left(f_{0}\right)$ if assumption (8.1) holds. This is true for all singularities with modality 0,1 and 2 . For the bimodal series and the quadrangle singularities, it is proved in this paper.

In [He99] the second author defined a classifying space $D_{B L}$ for Brieskorn like lattices (i.e. for objects which are sufficiently similar to the Brieskorn lattice $H_{0}^{\prime \prime}\left(f_{0}\right)$, see section 7 before theorem 7.11 for details). The group $G_{\mathbb{Z}}\left(f_{0}\right)$ acts properly discontinuously on it. The elements of $D_{B L}\left(f_{0}\right)$ are marked Brieskorn like lattices, and the elements of $D_{B L}\left(f_{0}\right) / G_{\mathbb{Z}}\left(f_{0}\right)$ are isomorphism classes of Brieskorn like lattices. One obtains a holomorphic period map

$$
B L: M_{\mu}^{\operatorname{mar}}\left(f_{0}\right) \rightarrow D_{B L}\left(f_{0}\right) .
$$

By [He02, Theorem 12.8] it is $G_{\mathbb{Z}}\left(f_{0}\right)$-equivariant, and it is an immersion (this fact is an infinitesimal Torelli type result). Now the following Torelli type conjectures are natural. Part (a) is for marked singularities. Part (b) recasts the Torelli type conjecture in [He93]. Part (a) implies part (b).

Conjecture 1.1. (a) [He11, Conjecture 5.3] The map BL is injective.

(b) $\left[\right.$ He93, Kap. 2 d)] The map $B L / G_{\mathbb{Z}}\left(f_{0}\right): M_{\mu}\left(f_{0}\right) \rightarrow$ $D_{B L}\left(f_{0}\right) / G_{\mathbb{Z}}\left(f_{0}\right)$ is injective. 
Theorem 1.2. (He93] He11] GH17] and the theorems 9.1 and 10.1 in this paper) Both Torelli type conjectures are true for all singularities with modality 0, 1 and 2.

The proofs have in almost all cases two parts:

(1) A good control of an (often multivalued) period map $T \rightarrow$ $D_{B L}\left(f_{0}\right)$, where $T$ is the parameter space of a well chosen family of normal forms.

(2) A good control of $G_{\mathbb{Z}}(f)$ and its action on $M_{\mu}^{\text {mar }}\left(f_{0}\right)$ and $D_{B L}\left(f_{0}\right)$.

In all cases, (1) is less work than (2). For the ADE-singularities, (1) is empty as there $T$ is a point, but (2) is not.

Part (b) of conjecture 1.1 was proved in He93 for the unimodal and bimodal singularities except seven of the eight series. For the seven series, the second author had unpublished calculations shortly after He93. But for technical reasons, part (b) stayed open for the subseries $S_{1,10 r}^{\sharp}, S_{1,10 r}, Z_{1,14 r}$. [He93] and these unpublished calculations give (1) and a part of (2).

In view of these old results, the major point in [He11, GH17] and in this paper is (2). But also some refinement of (1) is needed in the case of the singularities in this paper. The refinement is used for a better control of the transversal monodromy of the family of normal forms.

Finally, the conjecture $G_{\mathbb{Z}}\left(f_{0}\right)=G^{\text {mar }}\left(f_{0}\right)$ is probably wrong in general as it is wrong for the subseries with $m \mid p$. But for all singularities with modality 0, 1 and 2 except the eight series, the Torelli result for marked singularities and (1.3) require $G_{\mathbb{Z}}\left(f_{0}\right)=G^{\text {mar }}\left(f_{0}\right)$ to be true, as $B L$ is an immersion and there $\operatorname{dim} M_{\mu}^{\operatorname{mar}}\left(f_{0}\right)=\operatorname{modality}\left(f_{0}\right)=$ $\operatorname{dim} D_{B L}\left(f_{0}\right)$. And there $G_{\mathbb{Z}}\left(f_{0}\right)=G^{\text {mar }}\left(f_{0}\right)$ holds indeed. For the eight series, $\operatorname{dim} D_{B L}\left(f_{0}\right)>\operatorname{dim} M_{\mu}^{\text {mar }}\left(f_{0}\right)$, so there is enough space in $D_{B L}$ for infinitely many copies of $\left(M_{\mu}^{\text {mar }}\left(f_{0}\right)\right)^{0}$.

Open questions are now how to control the subgroup $G^{\text {mar }}\left(f_{0}\right) \subset$ $G_{\mathbb{Z}}\left(f_{0}\right)$ in general, and how to attack the Torelli conjectures in greater generality. For the second question, we plan to thicken $M_{\mu}^{\text {mar }}\left(f_{0}\right)$ to a $\mu$-dimensional $F$-manifold $M^{\text {mar }}\left(f_{0}\right)$ which is locally at each point of $M_{\mu}^{\text {mar }}\left(f_{0}\right)$ the base space of a universal unfolding. Then we will try to embed the Torelli type conjecture for $M_{\mu}^{m a r}$ into a family of Torelli type conjectures for all the $\mu$-homotopy strata of multigerms of singularities in $M^{\text {mar }}\left(f_{0}\right)$. We hope that this global point of view and the different geometry there with Stokes structures will give us new techniques. But this is a hope for the future. 
1.3. Structure of the paper. Section 2 is a collection of techniques which are useful to control the automorphisms of a pair $(\Lambda, L)$ or a pair $\left(\Lambda, M_{h}\right)$ where $\Lambda$ is a $\mathbb{Z}$-lattice, $L$ is a unimodular bilinear form and $M_{h}$ is an automorphism of finite order. We define Orlik blocks and study their automorphisms (lemma 2.8 will be very useful), and we cite classical algebraic facts on unit roots $\zeta$ and the rings $\mathbb{Z}[\zeta]$. All this is needed for the control of $G_{\mathbb{Z}}\left(f_{0}\right)$ in the sections 5 and 6 .

Section 3 discusses infinite Fuchsian groups which arise as subgroups of groups $G L(2, \mathbb{Z}[\zeta])$ with $\zeta$ a unit root. They are in fact arithmetic Fuchsian groups. But our treatment is essentially self-contained. Solutions of Pell equations with coefficients in $\mathbb{Z}[\zeta]$ play a role. For the quadrangle singularities, we need a precise analysis of some of these groups. They are certain triangle groups.

Section 4 recalls some classical notions and facts around singularities: Milnor fibration, Milnor lattice $M l(f)$, monodromy $M_{h}$, Seifert form $L$, Coxeter-Dynkin diagram, Stokes matrix, Thom-Sebastiani type results, suspension, polarized mixed Hodge structure on $H_{\mathbb{C}}^{\infty}$, its polarizing form.

Section 5 is long. It studies $G_{\mathbb{Z}}\left(f_{0}\right)$ for the eight bimodal series. Theorem 5.1 states the results. We start with a distinguished basis of the Milnor lattice with Coxeter-Dynkin diagram in Eb81. We calculate the monodromy $M_{h}$ and find 2 or 3 (3 only for $Z_{1, p}$ ) Orlik blocks whose direct sum is of index 1 or 2 in $M l\left(f_{0}\right)$. Then $G_{\mathbb{Z}}\left(f_{0}\right)$ is studied using these Orlik blocks and their rigidity and the results from the sections 2 and 3. A lot of calculations are needed, the different series behave differently. The singularities in the families $Q_{2, p}, W_{1,6 s-3}, S_{1,10}$ need special care.

Section 6 gives similar results for $G_{\mathbb{Z}}\left(f_{0}\right)$ for the quadrangle singularities. Theorem 6.1 states the results. Many, but not all, calculations and arguments in section 5 are also valid in section 6. Therefore this section is much shorter.

Section 7 gives a rather complete account on the Gauss-Manin connection and the Brieskorn lattice $H_{0}^{\prime \prime}(f)$ of a singularity $f$. It does not rewrite the proofs in Br70 and other papers, but it cites almost all known results. A highlight is the treatment of the bilinear forms. The polarizing form of the polarized mixed Hodge structure is connected with the restriction of K. Saito's higher residue pairings to $H_{0}^{\prime \prime}(f)$ and with Pham's intersection form for Lefschetz thimbles. We need the Fourier-Laplace transform $F L\left(H_{0}^{\prime \prime}(f)\right)$ for a Thom-Sebastiani formula for Brieskorn lattices. We need this in the special case of a suspension $f\left(z_{0}, \ldots, z_{n}\right)+z_{n+1}^{2}$ because we want to treat the suspensions in a more conceptual way than in [He93] He11] GH17]. 
Section 8 reviews the notions and results from [He11, the (strongly) marked singularities and their moduli spaces $M_{\mu}^{\text {smar }}\left(f_{0}\right)$ and $M_{\mu}^{\text {mar }}\left(f_{0}\right)$, the $\mu$-constant monodromy groups $G^{\text {smar }}\left(f_{0}\right)$ and $G^{\text {mar }}\left(f_{0}\right)$, and the Torelli conjectures. Corollary 8.14 is an application of the ThomSebastiani result for $F L\left(H_{0}^{\prime \prime}(f)\right)$ in section 7 and states that the marked Torelli conjecture for $f_{0}$ is equivalent to the marked Torelli conjecture for $f_{0}\left(z_{0}, \ldots, z_{n}\right)+\sum_{j=n+1}^{m} z_{j}^{2}$ for any fixed $m \geq n+1$. This allows us to consider in the sections 9 and 10 only the surface singularities.

Section 9 proves the marked Torelli conjecture for the bimodal series (theorem 9.1). It establishes the good control (1) of the multivalued period map $T \rightarrow D_{B L}\left(f_{0}\right)$ where $T=\mathbb{C}^{*} \times \mathbb{C}$ is the parameter space of normal forms in AGV85. Theorem 5.1 provides crucial information on $G_{\mathbb{Z}}\left(f_{0}\right)$.

Section 10 proves the marked Torelli conjecture for the quadrangle singularities (theorem 10.1). It starts with a careful own choice of normal forms with parameter space $T=(\mathbb{C}-\{0,1\}) \times \mathbb{C}$. It establishes the good control (1) of the multivalued period map $T \rightarrow D_{B L}\left(f_{0}\right)$. Theorem 6.1 provides crucial information on $G_{\mathbb{Z}}\left(f_{0}\right)$.

\section{2. $\mathbb{Z}$-LATTICES WITH UNIMODAL BILINEAR FORM AND MONODROMY}

This section provides tools for the study of the Milnor lattices with Seifert form and monodromy for the bimodal series and the quadrangle singularities, in the sections 5 and 6 . These lattices turn out to be quite rigid and to have rather few automorphisms. This is important for the global Torelli results in the sections 9 and 10 . This section puts together elementary, but nontrivial observations about $\mathbb{Z}$-lattices with a unimodal bilinear form and an (induced) monodromy.

Let $\Lambda$ be a $\mathbb{Z}$-lattice of rank $\mu \in \mathbb{Z}_{\geq 1}$, i.e. a free $\mathbb{Z}$-module of rank $\mu$. Let $L: \Lambda \times \Lambda \rightarrow \mathbb{Z}$ be a unimodal bilinear form, i.e. for any basis $\delta_{1}, \ldots, \delta_{\mu}$ we have $\operatorname{det}\left(L\left(\delta_{i}, \delta_{j}\right)_{i, j=1, \ldots, \mu}\right)= \pm 1$. We do not suppose that $L$ is symmetric or antisymmetric. Let $M_{h}: \Lambda \rightarrow \Lambda$ be the automorphism which is uniquely determined by

$$
L\left(M_{h}(a), b\right)=-L(b, a) \quad \text { for } a, b \in \Lambda .
$$

We call $L$ the Seifert form and $M_{h}$ the monodromy. 2.6 implies

$$
L\left(M_{h}(a), M_{h}(b)\right)=L(a, b),
$$

i.e. $L$ is $M_{h}$-invariant. We make the assumption that

$$
M_{h} \text { is finite, }
$$

i.e. $M_{h}$ is semisimple and its eigenvalues are unit roots. Then the characteristic polynomial $p_{\Lambda}$ of $M_{h}$ is a product of cyclotomic polynomials. 
Notations 2.1. (a) For any subring $R \subset \mathbb{C}$ denote $\Lambda_{R}:=\Lambda \otimes_{\mathbb{Z}} R$. For any monodromy invariant subspace $V \subset \Lambda_{\mathbb{C}}$ denote by $E(V) \subset S^{1}$ the set of eigenvalues of $M_{h}$ on $V$ and by $p_{V}$ its characteristic polynomial. For $\lambda \in E(V)$ denote $V_{\lambda}:=\operatorname{ker}\left(M_{h}-\lambda\right.$ id $\left.: V \rightarrow V\right) \subset V$. For any monodromy invariant sublattice $\Lambda^{(1)} \subset \Lambda$ write $E\left(\Lambda^{(1)}\right):=E\left(\Lambda_{\mathbb{C}}^{(1)}\right)$ and $p_{\Lambda^{(1)}}:=p_{\Lambda_{\mathbb{C}}^{(1)}}$ and $\Lambda_{\lambda}^{(1)}:=\left(\Lambda_{\mathbb{C}}^{(1)}\right)_{\lambda}$. For any product $p \in \mathbb{Z}[t]$ of cyclotomic polynomials with $p \mid p_{\Lambda^{(1)}}$ denote

$$
\Lambda_{\mathbb{C}, p}^{(1)}:=\bigoplus_{\lambda: p(\lambda)=0} \Lambda_{\lambda}^{(1)} \quad \text { and } \quad \Lambda_{p}^{(1)}:=\Lambda_{\mathbb{C}, p}^{(1)} \cap \Lambda^{(1)} .
$$

Then $\Lambda_{p}^{(1)}$ is a primitive and monodromy invariant sublattice of $\Lambda^{(1)}$.

(b) Recall that a sublattice $\Lambda^{(1)}$ of $\Lambda$ is primitive (in $\Lambda$ ) if and only if $\Lambda / \Lambda^{(1)}$ has no torsion and that for any sublattice $\Lambda^{(2)} \subset \Lambda$ there is a unique primitive sublattice $\Lambda^{(3)}$ with $\Lambda_{\mathbb{Q}}^{(3)}=\Lambda_{\mathbb{Q}}^{(2)}$, that it is $\Lambda^{(3)}=$ $\Lambda_{\mathbb{Q}}^{(2)} \cap \Lambda$ and that $\left[\Lambda^{(3)}: \Lambda^{(2)}\right]<\infty$.

(c) For $n \in \mathbb{Z}_{\geq 1}$, the cyclotomic polynomial $\Phi_{n}$ is

$$
\Phi_{n}=\prod_{\lambda: \operatorname{ord}(\lambda)=n}(t-\lambda) .
$$

It is unitary and in $\mathbb{Z}[t]$ and irreducible in $\mathbb{Z}[t]$ and $\mathbb{Q}[t]$.

(d) We define the square root on $S^{1}-\{-1\}$ by $\sqrt{e^{2 \pi i \alpha}}:=e^{\pi i \alpha}$ for $\alpha \in]-\frac{1}{2}, \frac{1}{2}[$.

Lemma 2.2. (a) Let $\lambda \in E(\Lambda)-\{1\}$. Then the sesquilinear (=linear $\times$ semilinear) form $h_{\lambda}: \Lambda_{\lambda} \times \Lambda_{\lambda} \rightarrow \mathbb{C}$ with

$$
h_{\lambda}(a, b):=\sqrt{-\lambda} \cdot L(a, \bar{b})
$$

is hermitian, i.e. $h_{\lambda}(b, a)=\overline{h_{\lambda}(a, b)}$. Especially, $\sqrt{-\lambda} \cdot L(a, \bar{a}) \in \mathbb{R}$. Together, these forms define a hermitian form $h:=\bigoplus_{\lambda \in E(\Lambda)-\{1\}} h_{\lambda}$.

(b) Let $V \subset \Lambda_{\mathbb{C}}$ be a monodromy invariant subspace with $1 \notin E(V)$. The following two properties are equivalent.

( $\alpha)\left.h\right|_{V}$ is positive definite.

( $\beta)$ The hermitian form on $V$ defined by $(a, b) \mapsto L(a, \bar{b})+L(\bar{b}, a)$ is positive definite.

Proof: (a) For $a, b \in V_{\lambda}$

$$
\begin{aligned}
\sqrt{-\lambda} \cdot L(b, \bar{a}) & =-\sqrt{-\lambda} \cdot L\left(M_{h}(\bar{a}), b\right)=-\sqrt{-\lambda} \cdot \bar{\lambda} \cdot L(\bar{a}, b) \\
& =\sqrt{-\bar{\lambda}} \cdot \overline{L(a, \bar{b})}=\overline{\sqrt{-\lambda} \cdot L(a, \bar{b})} .
\end{aligned}
$$


(b) Consider some $\lambda \in E(V)$. Observe $\sqrt{-\lambda}+\sqrt{-\bar{\lambda}}>0$ and for $a, b \in V$

$$
\begin{aligned}
L(a, \bar{b})+L(\bar{b}, a) & =L(a, \bar{b})+\overline{L(b, \bar{a})} \\
& =\sqrt{-\bar{\lambda}} \cdot h_{\lambda}(a, b)+\overline{\sqrt{-\bar{\lambda}} \cdot h_{\lambda}(b, a)} \\
& =(\sqrt{-\bar{\lambda}}+\sqrt{-\lambda}) \cdot h_{\lambda}(a, b) .
\end{aligned}
$$

Remarks 2.3. (i) The surface singularities considered in this paper do not have 1 as an eigenvalue of their monodromy. Therefore we do not treat it here.

(ii) Part (b) of lemma 2.2 connects to the polarization of the polarized Hodge structure of these surface singularities and rewrites it in different ways. $(\beta)$ is the classical way, with $-L-L^{t}$ on $\Lambda_{\mathbb{R}}$ as intersection form and $L+L^{t}$ as polarizing form. And $(\alpha)$ is the way used in the sections 3. 5 and 6 .

In 1972 Orlik formulated the beautiful conjecture 2.5 below on the integral monodromy of quasihomogeneous singularities Or72. It is known to be true for the quasihomogeneous curve singularities [MW86] and for the quasihomogeneous singularities with modality $\leq 2$ [He95]. But it is open for most other quasihomogeneous singularities.

A key observation for the treatment of the Milnor lattices of the bimodal series singularities and the quadrangle singularities is that they all have a structure close to Orlik's conjecture. The following definition gives the ingredients.

Definition 2.4. Let $\left(\Lambda, L, M_{h}\right)$ be as above. An Orlik block is a primitive and monodromy invariant sublattice $\Lambda^{(1)} \subset \Lambda$ with $\Lambda^{(1)} \supsetneqq\{0\}$ and with a cyclic generator, i.e. a lattice vector $e^{(1)} \in \Lambda^{(1)}$ with

$$
\Lambda^{(1)}=\bigoplus_{j=0}^{\operatorname{deg} p_{\Lambda^{(1)}}-1} \mathbb{Z} \cdot M_{h}^{j}\left(e^{(1)}\right)
$$

Conjecture 2.5. Or72, conjecture 3.1] Let $\left(\Lambda, M_{h}\right)$ be the Milnor lattice with monodromy of a quasihomogeneous singularity. Let $k:=$ $\max \left(\operatorname{dim} \Lambda_{\lambda} \mid \lambda \in E(\Lambda)\right)$. Then a decomposition $\Lambda=\bigoplus_{j=1}^{k} \Lambda^{(k)}$ into Orlik blocks $\Lambda^{(1)}, \ldots, \Lambda^{(k)}$ with $p_{\Lambda^{(j+1)}} \mid p_{\Lambda^{(j)}}$ for $0 \leq j<k$ exists.

Remarks 2.6. (i) A cyclic monodromy module has only one Jordan block for each eigenvalue. In this paper $M_{h}$ is semisimple. Therefore in an Orlik block, each eigenvalue has multiplicity one. 
(ii) In Orlik's conjecture 2.5, the polynomials $p_{\Lambda^{(1)}}, \ldots, p_{\Lambda^{(k)}}$ are unique. They are

$$
p_{\Lambda^{(j)}}=\prod_{\lambda \in E(\Lambda): \operatorname{dim} \Lambda_{\lambda} \geq j}(t-\lambda) \quad \text { for } j=1, \ldots, k .
$$

(iii) In the sections 5 and 6 , we will work most often with two Orlik blocks $\Lambda^{(1)}$ and $\Lambda^{(2)}$ such that $\Lambda^{(1)}+\Lambda^{(2)}=\Lambda^{(1)} \oplus \Lambda^{(2)}$ and that it is either equal to $\Lambda$ or has index 2 in $\Lambda$ and such that $L\left(\Lambda^{(1)}, \Lambda^{(2)}\right)=$ $L\left(\Lambda^{(2)}, \Lambda^{(1)}\right)=0$.

(iv) In all cases in section 5 with $\left[\Lambda: \Lambda^{(1)} \oplus \Lambda^{(2)}\right]=2$ except $S_{1,10}$, we will show

$$
\operatorname{Aut}(\Lambda, L)=\operatorname{Aut}\left(\Lambda^{(1)} \oplus \Lambda^{(2)}, L\right) .
$$

In many of these cases, there is an element $\gamma_{5} \in \Lambda_{\Phi_{2}}^{(1)}-\{0\}$ which is mapped by any element $g$ of $\operatorname{Aut}(\Lambda, L) \cup \operatorname{Aut}\left(\Lambda^{(1)} \oplus \Lambda^{(2)}, L\right)$ to $\pm \gamma_{5}$ and such that

$$
\Lambda^{(1)} \oplus \Lambda^{(2)}=\left\{a \in \Lambda \mid L\left(a, \gamma_{5}\right) \in 2 \mathbb{Z}\right\} .
$$

Then any $g \in \operatorname{Aut}(\Lambda, L)$ maps $\Lambda^{(1)} \oplus \Lambda^{(2)}$ to itself, so $\operatorname{Aut}(\Lambda, L) \subset$ $\operatorname{Aut}\left(\Lambda^{(1)} \oplus \Lambda^{(2)}, L\right)$.

If this inclusion $\subset$ holds, the following argument shows that $\operatorname{Aut}(\Lambda, L)$ is either equal to or a subgroup of index 2 in $\operatorname{Aut}\left(\Lambda^{(1)} \oplus\right.$ $\left.\Lambda^{(2)}, L\right)$. Unfortunately it looks hard to exclude the second case. Therefore in section 5 we show the equality 2.13) in a different (and more laborious) way.

Let $\Lambda^{(0)} \subset \Lambda_{\mathbb{Q}}$ be the unique lattice such that

$$
L: \Lambda^{(0)} \times\left(\Lambda^{(1)} \oplus \Lambda^{(2)}\right) \rightarrow \mathbb{Z}
$$

is unimodal. Then $\Lambda^{(0)} \supset \Lambda \supset \Lambda^{(1)} \oplus \Lambda^{(2)}$ and $\left[\Lambda^{(0)}: \Lambda\right]=2$ and $\operatorname{Aut}\left(\Lambda^{(1)} \oplus \Lambda^{(2)}, L\right)=\operatorname{Aut}\left(\Lambda^{(0)}, L\right)$.

1st case, $\Lambda^{(0)} /\left(\Lambda^{(1)} \oplus \Lambda^{(2)}\right) \cong \mathbb{Z} / 4 \mathbb{Z}$. Then $\Lambda$ is the unique lattice between $\Lambda^{(0)}$ and $\Lambda^{(1)} \oplus \Lambda^{(2)}$ with $\left[\Lambda^{(0)}: \Lambda\right]=2$. Then any $g \in \operatorname{Aut}\left(\Lambda^{(1)} \oplus \Lambda^{(2)}, L\right)$ respects $\Lambda$, so 2.13 holds.

2nd case, $\Lambda^{(0)} /\left(\Lambda^{(1)} \oplus \Lambda^{(2)}\right) \cong \mathbb{Z} / 2 \mathbb{Z} \times \mathbb{Z} / 2 \mathbb{Z}$. Then there are three lattices between $\Lambda^{(0)}$ and $\Lambda^{(1)} \oplus \Lambda^{(2)}$ with index 2 in $\Lambda^{(0)}$, one for each subgroup of index 2 in $\mathbb{Z} / 2 \mathbb{Z} \times \mathbb{Z} / 2 \mathbb{Z}$. One of them is $\Lambda$. Another one is $\left\{a \in \Lambda^{(0)} \mid L\left(a, \gamma_{5}\right) \in 2 \mathbb{Z}\right\}$. No element of $\operatorname{Aut}\left(\Lambda^{(0)}, L\right)$ maps $\Lambda$ to this lattice. But it looks hard to exclude the possibility that half of the elements of $\operatorname{Aut}\left(\Lambda^{(0)}, L\right)$ map $\Lambda$ to the third lattice between $\Lambda^{(0)}$ and $\Lambda^{(1)} \oplus \Lambda^{(2)}$. 
(v) If $\Lambda^{(1)} \subset \Lambda$ is an Orlik block with cyclic generator $e^{(1)}$ and if $p_{\Lambda^{(1)}}=p_{1} \cdot p_{2}$ with $\operatorname{deg} p_{1} \geq 1$ and $\operatorname{deg} p_{2} \geq 1$, then the sublattice $\Lambda^{(2)}:=\Lambda_{p_{1}}^{(1)}$ is also an Orlik block, and a cyclic generator is

$$
e^{(2)}:=p_{2}\left(M_{h}\right)\left(e^{(1)}\right)
$$

(vi) If $\Lambda^{(1)} \subset \Lambda$ is an Orlik block with generator $e^{(1)}$ and $\lambda \in E\left(\Lambda^{(1)}\right)$ is an eigenvalue of the monodromy on $\Lambda^{(1)}$, then an eigenvector is

$$
v\left(e^{(1)}, \lambda\right):=\frac{p_{\Lambda^{(1)}}}{t-\lambda}\left(M_{h}\right)\left(e^{(1)}\right) .
$$

And then

$$
\begin{aligned}
& L\left(v\left(e^{(1)}, \lambda\right), v\left(e^{(1)}, \bar{\lambda}\right)\right) \\
= & L\left(v\left(e^{(1)}, \lambda\right), \frac{p_{\Lambda^{(1)}}}{t-\bar{\lambda}}\left(M_{h}\right)\left(e^{(1)}\right)\right) \\
= & L\left(\frac{p_{\Lambda^{(1)}}}{t-\bar{\lambda}}\left(M_{h}^{-1}\right) v\left(e^{(1)}, \lambda\right), e^{(1)}\right) \\
= & \frac{p_{\Lambda^{(1)}}}{t-\bar{\lambda}}(\bar{\lambda}) \cdot L\left(v\left(e^{(1)}, \lambda\right), e^{(1)}\right) \\
= & \frac{p_{\Lambda^{(1)}}}{t-\bar{\lambda}}(\bar{\lambda}) \cdot L\left(\frac{p_{\Lambda^{(1)}}}{t-\lambda}\left(M_{h}\right)\left(e^{(1)}\right), e^{(1)}\right) .
\end{aligned}
$$

This calculation will be useful in section 5 .

The following two lemmata concern automorphisms of sums of Orlik blocks (lemma 2.7) or of a single Orlik block (lemma 2.8). They will be useful tools in order to show the rigidity of the Milnor lattices in the sections 5 and 6 .

Lemma 2.7. Let $\left(\Lambda, M_{h}\right)$ be as above (we will not need $L$ here, only $\left.M_{h}\right)$. Let $\Lambda^{(1)}, \ldots, \Lambda^{(k)} \subset \Lambda$ be Orlik blocks with cyclic generators $e^{(1)}, \ldots, e^{(k)}$ and with

$$
\Lambda^{(1)}+\ldots+\Lambda^{(k)}=\Lambda^{(1)} \oplus \ldots \oplus \Lambda^{(k)} .
$$

Consider an element $g \in \operatorname{Aut}\left(\Lambda^{(1)} \oplus \ldots \oplus \Lambda^{(k)}, M_{h}\right)$. Then there are unique polynomials $p_{i j} \in \mathbb{Z}[t]_{<\operatorname{rank} \Lambda^{(j)}}$ for $i, j=1, \ldots, k$ with

$$
g\left(e^{(j)}\right)=\sum_{i=1}^{k} p_{i j}\left(M_{h}\right)\left(e^{(i)}\right) .
$$

Suppose now that $p_{0} \in \mathbb{Z}[t]$ divides $\operatorname{gcd}\left(p_{\Lambda^{(1)}}, \ldots, p_{\Lambda^{(k)}}\right)$ and that

$$
g=\text { id on } \Lambda_{p_{\Lambda}(j) / p_{0}}^{(j)} \quad \text { for any } j
$$


so that $g$ acts nontrivial only on $\left(\Lambda^{(1)} \oplus \ldots \oplus \Lambda^{(k)}\right)_{p_{0}}$. Then

$$
p_{i j}=\delta_{i j}+\frac{p_{\Lambda^{(i)}}}{p_{0}} \cdot q_{i j}
$$

for suitable polynomials $q_{i j} \in \mathbb{Z}[t]_{<\operatorname{deg} p_{0}}$.

Suppose furthermore that a unit root $\xi$ satisfies $p_{0}(\xi)=0$. Then $g$ with respect to the eigenvectors $v\left(e^{(1)}, \xi\right) \in \Lambda_{\xi}^{(1)}, \ldots, v\left(e^{(k)}, \xi\right) \in \Lambda_{\xi}^{(k)}$ (defined in (2.16) ) is given by

$$
g\left(v\left(e^{(j)}, \xi\right)\right)=\sum_{i=1}^{k}\left(\delta_{i j}+\frac{p_{\Lambda^{(j)}}}{p_{0}} \cdot q_{i j}\right)(\xi) \cdot v\left(e^{(i)}, \xi\right)
$$

Proof: Only the part after 2.18) is nontrivial. Suppose that $p_{0}$ and $g$ are as stated above. By assumption

$$
\begin{aligned}
g\left(e^{(j)}\right)-e^{(j)} & \in\left(\Lambda^{(1)} \oplus \ldots \oplus \Lambda^{(k)}\right)_{p_{0}} \\
& \subset \bigoplus_{i=1}^{k} \Lambda_{\mathbb{C}, p_{0}}^{(i)}=\bigoplus_{i=1}^{k} \frac{p_{\Lambda^{(i)}}}{p_{0}}\left(M_{h}\right)\left(\Lambda_{\mathbb{C}}^{(i)}\right) .
\end{aligned}
$$

Thus $p_{i j}-\delta_{i j} \in \frac{p_{\Lambda^{(i)}}}{p_{0}} \cdot \mathbb{C}[t]$, thus $p_{i j}-\delta_{i j} \in \frac{p_{\Lambda^{(i)}}}{p_{0}} \cdot \mathbb{Z}[t]_{<\operatorname{deg} p_{0}}$.

The following calculation proves (2.21).

$$
\begin{aligned}
g\left(v\left(e^{(j)}, \xi\right)\right) & =g\left(\frac{p_{\Lambda^{(j)}}}{t-\xi}\left(M_{h}\right)\left(e^{(j)}\right)\right)=\frac{p_{\Lambda^{(j)}}}{t-\xi}\left(M_{h}\right)\left(g\left(e^{(j)}\right)\right) \\
& =\frac{p_{\Lambda^{(j)}}}{t-\xi}\left(M_{h}\right)\left(\sum_{i=1}^{k}\left(\delta_{i j}+\frac{p_{\Lambda^{(i)}}}{p_{0}} \cdot q_{i j}\right)\left(M_{h}\right)\left(e^{(i)}\right)\right) \\
& =\sum_{i=1}^{k}\left(\left(\delta_{i j}+\frac{p_{\Lambda^{(i)}}}{p_{0}} \cdot q_{i j}\right) \cdot \frac{p_{\Lambda^{(j)}}}{t-\xi}\right)\left(M_{h}\right)\left(e^{(i)}\right) \\
& =\sum_{i=1}^{k}\left(\delta_{i j}+\frac{p_{\Lambda^{(j)}}}{p_{0}} \cdot q_{i j}\right)\left(M_{h}\right)\left(v\left(e^{(i)}, \xi\right)\right) \\
& =\sum_{i=1}^{k}\left(\delta_{i j}+\frac{p_{\Lambda^{(j)}}}{p_{0}} \cdot q_{i j}\right)(\xi) \cdot v\left(e^{(i)}, \xi\right) .
\end{aligned}
$$

The following lemma is cited from [He11, lemma 8.2], but it goes back to arguments in [He98, ch. 6].

Lemma 2.8. Let $\left(\Lambda, L, M_{h}\right)$ be as above. Suppose that $\Lambda$ is a single Orlik block. 
We make the following nontrivial assumption on the set

$$
\text { Ord }:=\left\{\operatorname{ord} \lambda \mid \lambda \text { eigenvalue of } M_{h}\right\} \subset \mathbb{Z}_{\geq_{1}}
$$

of orders of the eigenvalues of the monodromy $M_{h}$ : There exist four sequences $\left(m_{i}\right)_{i=1, \ldots, \mid \text { Ord } \mid},(j(i))_{i=2, \ldots, \mid \text { Ord } \mid},\left(p_{i}\right)_{i=2, \ldots, \mid \text { Ord } \mid},\left(k_{i}\right)_{i=2, \ldots, \mid \text { Ord } \mid}$ of numbers in $\mathbb{Z}_{\geq 1}$ and two numbers $i_{1}, i_{2} \in \mathbb{Z}_{\geq 1}$ with $i_{1} \leq i_{2} \leq \mid$ Ord $\mid$ and with the properties:

$$
\begin{aligned}
& \text { Ord }=\left\{m_{1}, \ldots, m_{\mid \text {Ord } \mid}\right\}, \\
& p_{i} \text { is a prime number, } p_{i}=2 \text { for } i_{1}+1 \leq i \leq i_{2}, p_{i} \geq 3 \text { else, } \\
& j(i)=i-1 \text { for } i_{1}+1 \leq i \leq i_{2}, j(i)<i \text { else, } \\
& m_{i}=m_{j(i)} / p_{i}^{k_{i}} .
\end{aligned}
$$

Then

$$
\operatorname{Aut}\left(\Lambda, L, M_{h}\right)=\left\{ \pm M_{h}^{k} \mid k \in \mathbb{Z}\right\} .
$$

We will need some basic facts for the unit $\operatorname{roots} \zeta=e^{2 \pi i / m}$ with $m \in\{10,12,14,18\}$. The following theorem 2.9 collects some facts for general unit roots. Theorem 2.10 cites two classical results on orders in algebraic number fields. Lemma 2.11 puts together some specific properties for the unit roots of the orders $m \in\{10,12,14,18\}$.

Theorem 2.9. Fix $m \in \mathbb{Z}_{\geq 3}$ and define $\zeta:=e^{2 \pi i / m}, p_{1}:=\zeta+\bar{\zeta}$.

(a)

$$
\begin{aligned}
\operatorname{Eiw}(\zeta) & :=\left\{ \pm \zeta^{k} \mid k \in \mathbb{Z}\right\} \\
& =\{\text { unit roots in } \mathbb{Q}(\zeta)\}=\{\text { unit roots in } \mathbb{Z}[\zeta]\} \\
& =\{a \in \mathbb{Z}[\zeta]|| a \mid=1\} .
\end{aligned}
$$

(b) $\mathbb{Z}[\zeta]$ is the ring of algebraic integers of $\mathbb{Q}(\zeta)$.

(c) $\mathbb{Z}\left[p_{1}\right]$ is the ring of algebraic integers of $\mathbb{Q}\left(p_{1}\right)$. And $\mathbb{Q}\left(p_{1}\right)$ is the maximal real subfield of $\mathbb{Q}(\zeta)$.

(d) $\mathbb{Q}(\zeta)$ has class field number 1 and thus $\mathbb{Z}[\zeta]$ is a principal ideal domain if and only if $m \in A_{1} \cup A_{2} \cup A_{3}$ where

$$
\begin{aligned}
& A_{1}=\{1,3,5, \ldots, 21\} \cup\{25,27,33,35,45\}, \\
& A_{2}=\left\{2 n \mid n \in A_{1}\right\}, \\
& A_{3}=\left\{4 n \mid n \in A_{4}\right\}, \quad A_{4}=\{1,2,3, \ldots, 12\} \cup\{15,21\} .
\end{aligned}
$$

(e) If $\mathbb{Q}(\zeta)$ has class field number 1 , then $\mathbb{Q}\left(p_{1}\right)$ has class field number 1 and thus $\mathbb{Z}\left[p_{1}\right]$ is a principal ideal domain.

(f) $\zeta-1 \in(\mathbb{Z}[\zeta])^{*}$ if $m \notin\left\{p^{k} \mid p\right.$ a prime number, $\left.k \in \mathbb{Z}_{\geq 1}\right\}$.

$\zeta+1 \in(\mathbb{Z}[\zeta])^{*}$ if $m \notin\left\{2 \cdot p^{k} \mid p\right.$ a prime number, $\left.k \in \mathbb{Z}_{\geq 1}\right\}$.

Proof: (a) [Wa97] lemma 1.6 and exercise 2.3. (b) [Wa97] theorem 2.6. (c) Wa97 proposition 2.16. (d) [Wa97] theorem 11.1. (e) Wa97] theorem 4.10. (f) [Wa97] proposition 2.8. 
Theorem 2.10. Let $K$ be an algebraic number field of degree $n=s+2 t$ over $\mathbb{Q}$ with s real embeddings $\sigma_{j}: K \rightarrow \mathbb{R}, j=1, \ldots, s$, and $2 t$ complex embeddings $\sigma_{j}: K \rightarrow \mathbb{C}, j=s+1, \ldots, n$, with $\sigma_{s+t+j}=\overline{\sigma_{s+j}}$ for $j=1, \ldots, t$.

(a) BS66, Ch. 2, 3.1 Theorem 1] Define $\underline{\sigma}:=\left(\sigma_{1}, \ldots, \sigma_{s+t}\right): K \rightarrow$ $\mathbb{R}^{s} \times \mathbb{C}^{t} \cong \mathbb{R}^{n}$. Any $\mathbb{Q}$-basis of $K$ maps to an $\mathbb{R}$-basis of $\mathbb{R}^{n}$. Thus the image under $\underline{\sigma}$ of any order $\mathcal{O} \subset K$ is a lattice of rank $n$ in $\mathbb{R}^{n}$.

(b) (Dirichlet's unit theorem, [BS66, Ch. 2, 4.3 Theorem 5]) Let $\mathcal{O} \subset K$ be an order. One can choose $r=s+t-1$ units $a_{1}, \ldots, a_{r} \in \mathcal{O}^{*}$ such that any unit has a unique representation $\xi \cdot a_{1}^{k_{1}} \cdot \ldots \cdot a_{r}^{k_{r}}$ with $k_{1}, \ldots, k_{r} \in \mathbb{Z}$ and $\xi$ a root of 1 in $\mathcal{O}$.

Of course, $n=\varphi(m)=2 t$ in the case $\mathcal{O}=\mathbb{Z}[\zeta] \subset K=\mathbb{Q}(\zeta)$, and $n=\frac{\varphi(m)}{2}$ in the case $\mathcal{O}=\mathbb{Z}\left[p_{1}\right] \subset K=\mathbb{Q}\left[p_{1}\right]$, where $\zeta=e^{2 \pi i / m}$ and $p_{1}=\zeta+\bar{\zeta}$.

The unit roots of orders $m \in\{10,12,14,18\}$ are most important in this paper. The next lemma collects specific properties of $\mathbb{Z}[\zeta]$ for these orders.

Lemma 2.11. Fix $m \in\{10,12,14,18\}$ and define $\zeta=e^{2 \pi i / m}$ and $p_{1}=\zeta+\bar{\zeta}$.

$\mathbb{Z}[\zeta]$ and $\mathbb{Z}\left[p_{1}\right]$ are principal ideal domains (by theorem $2.9(d)+(e)$ ).

(a) $m=10: \Phi_{10}(t)=t^{4}-t^{3}+t^{2}-t+1$,

$$
\begin{aligned}
\mathbb{Z}[\zeta]^{*}= & \operatorname{Eiw}(\zeta) \cdot \mathbb{Z}\left[p_{1}\right]^{*} \supset\{\zeta-1\}, \\
\mathbb{Z}\left[p_{1}\right]^{*}= & \{ \pm 1\} \times\left\{p_{1}^{k} \mid k \in \mathbb{Z}\right\} \supset\left\{p_{1}-2, p_{1}-1, p_{1}, p_{1}+1\right\}, \\
p_{1}= & \frac{\sqrt{5}+1}{2}>0, \quad p_{3}:=\zeta^{3}+\bar{\zeta}^{3}=\frac{-\sqrt{5}+1}{2}<0, \\
\operatorname{Gal}\left(\mathbb{Q}\left(p_{1}\right): \mathbb{Q}\right)= & \{\operatorname{id}, \varphi\}, \quad \varphi: p_{1} \mapsto p_{3} \mapsto p_{1}, \\
\left(x-p_{1}\right)\left(x-p_{3}\right)= & x^{2}-x-1, \quad p_{1}+p_{3}=1, p_{1} p_{3}=-1, p_{1}^{2}=p_{1}+1 . \\
(b) m=12: \Phi_{12}(t) & =t^{4}-t^{2}+1, \\
\mathbb{Z}[\zeta]^{*} & =\operatorname{Eiw}(\zeta) \cdot \mathbb{Z}\left[p_{1}\right]^{*} \cup(\zeta+1) \cdot \operatorname{Eiw}(\zeta) \cdot \mathbb{Z}\left[p_{1}\right]^{*} \\
& =\operatorname{Eiw}(\zeta) \cdot\left\{(\zeta+1)^{k} \mid k \in \mathbb{Z}\right\} \supset\{\zeta-1, \zeta+1\}, \\
\mathbb{Z}\left[p_{1}\right]^{*} & =\{ \pm 1\} \times\left\{p_{1}^{k} \mid k \in \mathbb{Z}\right\} \supset\left\{p_{1}-2, p_{1}+2\right\}, \\
p_{1} & =\sqrt{3}>0, \quad p_{5}:=\zeta^{5}+\bar{\zeta}^{5}=-\sqrt{3}<0, \\
\operatorname{Gal}\left(\mathbb{Q}\left(p_{1}\right): \mathbb{Q}\right) & =\{\operatorname{id}, \varphi\}, \quad \varphi: p_{1} \mapsto p_{5} \mapsto p_{1}, \\
\left(x-p_{1}\right)\left(x-p_{5}\right) & =x^{2}-3, \quad p_{1}+p_{5}=0, p_{1} p_{5}=-3, p_{1}^{2}=3 .
\end{aligned}
$$




$$
\begin{aligned}
\text { (c) } m=14: \Phi_{14}(t)=t^{6} & -t^{5}+t^{4}-t^{3}+t^{2}-t+1, \\
\mathbb{Z}[\zeta]^{*} & =\operatorname{Eiw}(\zeta) \cdot \mathbb{Z}\left[p_{1}\right]^{*} \supset\{\zeta-1\}, \\
\mathbb{Z}\left[p_{1}\right]^{*} & =\{ \pm 1\} \times\left\{p_{1}^{k_{1}} p_{3}^{k_{3}} \mid k_{1}, k_{3} \in \mathbb{Z}\right\} \\
& \supset\left\{p_{1}-2, p_{1}-1, p_{1}, p_{1}+1\right\}, \\
p_{1} & >0, p_{3}:=\zeta^{3}+\bar{\zeta}^{3}>0, p_{5}:=\zeta^{5}+\bar{\zeta}^{5}<0, \\
\operatorname{Gal}\left(\mathbb{Q}\left(p_{1}\right): \mathbb{Q}\right) & =\left\{\mathrm{id}, \varphi, \varphi^{2}\right\}, \quad \varphi: p_{1} \mapsto p_{3} \mapsto p_{5} \mapsto p_{1}, \\
p_{1} p_{3} p_{5} & =-1, \quad p_{1} p_{3}=p_{1}-1, \quad p_{1}^{2}=-p_{5}+2 . \\
\left(x-p_{1}\right)\left(x-p_{3}\right)\left(x-p_{5}\right) & =x^{3}-x^{2}-2 x+1, \quad p_{1}+p_{3}+p_{5}=1, \\
(d) m=18: \Phi_{18}(t)=t^{6} & -t^{3}+1, \\
\mathbb{Z}[\zeta]^{*} & =\operatorname{Eiw}(\zeta) \cdot \mathbb{Z}\left[p_{1}\right]^{*} \supset\{\zeta-1\}, \\
\mathbb{Z}\left[p_{1}\right]^{*} & =\{ \pm 1\} \times\left\{p_{1}^{k_{1}} p_{5}^{k_{5}} \mid k_{1}, k_{5} \in \mathbb{Z}\right\} \\
& \supset\left\{p_{1}-2, p_{1}, p_{1}+1\right\}, \\
p_{1} & >0, p_{5}:=\zeta^{5}+\bar{\zeta}^{5}<0, p_{7}:=\zeta^{7}+\bar{\zeta}^{7}<0, \\
& \left\{\operatorname{id}, \varphi, \varphi^{2}\right\}, \quad \varphi: p_{1} \mapsto p_{5} \mapsto p_{7} \mapsto p_{1}, \\
\operatorname{Gal}\left(\mathbb{Q}\left(p_{1}\right): \mathbb{Q}\right) & =x^{3}-3 x-1, \quad p_{1}+p_{5}+p_{7}=0, \\
p_{1} p_{5} p_{7} & =1, \quad p_{1} p_{5}=-p_{5}-1, \quad p_{1}^{2}=-p_{7}+2 .
\end{aligned}
$$

Proof: That the index $\left[\mathbb{Z}[\zeta]^{*}: \operatorname{Eiw}(\zeta) \cdot \mathbb{Z}\left[p_{1}\right]^{*}\right]$ is 1 for $m \in$ $\{10,14,18\}$ and 2 for $m=12$, follows from [Wa97, theorem 4.12 and corollary 4.13]. That $\mathbb{Z}\left[p_{1}\right]^{*}$ is as stated, follows for $m \in\{10,14,18\}$ from [Wa97, theorem 8.2 and lemma 8.1 (a)]. For $m=12$ [Wa97, §8.1] is not so useful, but there the proof of $\mathbb{Z}\left[p_{1}\right]^{*}=\{ \pm 1\} \cdot\left\{p_{1}^{k} \mid k \in \mathbb{Z}\right\}$ is easy. Everything else is elementary.

Part (b) of the following lemma applies with $\Lambda=M l(f)$ and $\Lambda^{(1)}=$ $\widetilde{B}_{1} \oplus B_{2}$ (see the theorems 5.1 and 6.1 to most of the Milnor lattices in the sections 5 and 6 . We will need (2.24).

Lemma 2.12. (a) Let $p=\prod_{i \in I} \Phi_{m_{i}}$ be a product of cyclotomic polynomials. Then $p(1) \equiv 1(2)$ if and only if all $m_{i} \in \mathbb{Z}_{\geq 1}-\left\{2^{k} \mid k \in \mathbb{Z}_{\geq 0}\right\}$.

(b) Let $\left(\Lambda, L, M_{h}\right)$ be as above (we will not need $L$ here, only $M_{h}$ ). Let $\Lambda^{(1)} \subset \Lambda$ be an $M_{h}$-invariant sublattice with $\left[\Lambda: \Lambda^{(1)}\right]=2$. Write

$$
\begin{aligned}
p_{\Lambda}= & p_{1} \cdot p_{2} \quad \text { with } p_{j}=\prod_{m \in J_{j}} \Phi_{m} \\
\text { and } \quad & J_{1} \subset \mathbb{Z}_{\geq 1}-\left\{2^{k} \mid k \in \mathbb{Z}_{\geq 0}\right\}, \quad J_{1} \subset\left\{2^{k} \mid k \in \mathbb{Z}_{\geq 0}\right\} .
\end{aligned}
$$


Then $J_{2} \neq \emptyset, p_{2} \neq 1$, and

$$
\begin{aligned}
\Lambda_{p} & =\Lambda_{p}^{(1)} \quad \text { for any } p \text { with } p \mid p_{1}, \\
{\left[\Lambda_{p}: \Lambda_{p}^{(1)}\right] } & =2 \quad \text { for any } p \text { with } p_{2} \mid p .
\end{aligned}
$$

Proof: (a) Observe $\Phi_{2^{k}}(t)=t^{2^{k-1}}+1$ for $k \geq 1$ and

$$
t^{2^{k} \cdot q}-1=\left(t^{2^{k}}-1\right)\left(t^{2^{k}(q-1)}+t^{2^{k}(q-2)}+\ldots+t^{2^{k}}+1\right) .
$$

For odd $q>1$, the second factor has at $t=1$ the odd value $q$. Therefore $\Phi_{m}(1) \equiv 1(2)$ for any $m$ with $2^{k}|m| 2^{k} \cdot q$ and $2^{k} \neq m$ with $q$ odd.

(b) For an arbitrary element $\gamma \in \Lambda-\Lambda^{(1)}$,

$$
\Lambda-\Lambda^{(1)}=\gamma+\Lambda^{(1)} \text {. }
$$

This set is $M_{h}$-invariant because $\Lambda^{(1)}$ is $M_{h}$-invariant. Thus for any $k \in \mathbb{Z}_{\geq 1} M_{h}^{k}(\gamma) \in \Lambda-\Lambda^{(1)}$. By part (a) $p_{1}(1) \equiv 1(2)$. Thus $p_{1}\left(M_{h}\right)(\gamma) \in$ $\Lambda-\Lambda^{(1)}$ and

$$
p_{1}\left(M_{h}\right)\left(\Lambda-\Lambda^{(1)}\right) \subset \Lambda-\Lambda^{(1)} .
$$

On the other hand

$$
\begin{aligned}
p_{1}\left(M_{h}\right)\left(\Lambda_{p_{1}}\right) & =\{0\} \subset \Lambda^{(1)}, \text { thus } \Lambda_{p_{1}} \subset \Lambda^{(1)}, \text { thus } 2.24 . \\
p_{1}\left(M_{h}\right)(\Lambda) & \subset \Lambda_{p_{2}}, \text { thus } \Lambda_{p_{2}} \cap\left(\Lambda-\Lambda^{(1)}\right) \neq \emptyset, \text { thus } 2.25 .
\end{aligned}
$$

\section{SOME Fuchsian GROUPS}

Notations 3.1. For any $m \in \mathbb{Z}_{\geq 3}$ define $\zeta:=e^{2 \pi i / m}$ and $p_{1}:=\zeta+\bar{\zeta}$. The letter $\xi$ will denote in this section a primitive $m$-th unit root. An element of $\mathbb{Q}(\zeta)$ will be written as $a$ or $a(\zeta)$. Then $a(\xi)$ is the image $\varphi(a)$ for $\varphi \in \operatorname{Gal}(\mathbb{Q}(\zeta): \mathbb{Q})$ with $\varphi(\zeta)=\xi$.

Any element $A=\left(\begin{array}{ll}a & b \\ c & d\end{array}\right) \in G L(2, \mathbb{C})$ acts on $\mathbb{P}^{1} \mathbb{C}$ by the linear transformation $z \mapsto \frac{a z+b}{c z+d}$, which is an automorphism of $\mathbb{P}^{1} \mathbb{C}$. The limit set $L(\Gamma) \subset \mathbb{P}^{1} \mathbb{C}$ of a subgroup $\Gamma \subset G L(2, \mathbb{C})$ is [Le64, III 1B]

$$
\begin{aligned}
L(\Gamma)= & \left\{z \in \mathbb{P}^{1} \mathbb{C} \mid \exists z_{0} \in \mathbb{P}^{1} \mathbb{C} \text { and } \exists\right. \text { a sequence of different } \\
& \text { elements } \left.\gamma_{i} \in \Gamma \text { with } \gamma_{i}\left(z_{0}\right) \rightarrow z\right\} .
\end{aligned}
$$

A subgroup $\Gamma \subset G L(2, \mathbb{C})$ and the induced subgroup of $P G L(2, \mathbb{C})$ are called Fuchsian if $\Gamma$ maps a certain circle $C \subset \mathbb{P}^{1} \mathbb{C}$ to itself and $L(\Gamma) \subset$ $C$. By a theorem of Poincaré [Le64, III 3I], a subgroup $\Gamma \subset G L(2, \mathbb{C})$ is Fuchsian if it maps a certain circle $C \subset \mathbb{P}^{1} \mathbb{C}$ to itself and is discrete in $G L(2, \mathbb{C})$. 
In the sections 5 and 6 we will encounter Fuchsian groups which arise in the following way.

Theorem 3.2. Let $m \in \mathbb{Z}_{\geq 3}, \zeta:=e^{2 \pi i / m}, p_{1}:=\zeta+\bar{\zeta}$, and $w=w(\zeta) \in$ $\mathbb{Q}(\zeta)$ with

$$
\begin{array}{ll}
w(\zeta)>0 & \text { (thus } \left.w(\zeta)=w(\bar{\zeta}) \in \mathbb{Q}\left(p_{1}\right)\right), \\
w(\xi)<0 & \text { for any primitive } m \text {-th unit root } \xi \notin\{\zeta, \bar{\zeta}\} .
\end{array}
$$

Then the matrix group

$$
\Gamma:=\left\{A \in G L(2, \mathbb{Z}[\zeta]) \mid\left(\begin{array}{cc}
-1 & 0 \\
0 & w
\end{array}\right)=A^{t}\left(\begin{array}{cc}
-1 & 0 \\
0 & w
\end{array}\right) \bar{A}\right\}
$$

is an infinite Fuchsian group. It preserves the circle

$$
C=\left\{\left.z \in \mathbb{C}|| z\right|^{2}=w\right\} .
$$

The map

$$
\begin{array}{r}
\left\{(a, c, \delta) \in \mathbb{Z}[\zeta]^{2} \times\left.\operatorname{Eiw}(\zeta)|| a\right|^{2}-1=w \cdot|c|^{2}\right\} \rightarrow \Gamma \\
(a, c, \delta) \mapsto A:=\left(\begin{array}{cc}
a & w \cdot \bar{c} \cdot \delta \\
c & \bar{a} \cdot \delta
\end{array}\right)
\end{array}
$$

is a bijection (here $\operatorname{Eiw}(\zeta)=\left\{ \pm \zeta^{k} \mid k \in \mathbb{Z}\right\}$, see theorem 2.9 (a)).

Proof: The matrix $\left(\begin{array}{cc}-1 & 0 \\ 0 & w\end{array}\right)$ defines an indefinite hermitian form on $\mathbb{C}^{2}$. The isotropic lines are $\mathbb{C} \cdot\left(\begin{array}{l}z \\ 1\end{array}\right)$ with $z \in C$. Therefore any matrix $A \in \Gamma$ maps $C$ to itself.

The matrix equation which defines $\Gamma$ can be spelled out as follows,

$$
\begin{aligned}
\left(\begin{array}{cc}
-1 & 0 \\
0 & w
\end{array}\right) & =\left(\begin{array}{ll}
a & c \\
b & d
\end{array}\right)\left(\begin{array}{cc}
-1 & 0 \\
0 & w
\end{array}\right)\left(\begin{array}{ll}
\bar{a} & \bar{b} \\
\bar{c} & \bar{d}
\end{array}\right) \\
& =\left(\begin{array}{ll}
-a \bar{a}+w c \bar{c} & -a \bar{b}+w c \bar{d} \\
-\bar{a} b+w \bar{c} d & -b \bar{b}+w \bar{d}
\end{array}\right) .
\end{aligned}
$$

The determinant $\delta=\operatorname{det} A=a d-b c$ is in $\mathbb{Z}[\zeta]$ and has absolute value 1 , so it is in $\operatorname{Eiw}(\zeta)$ by theorem 2.9 (a). The equations above give

$$
\begin{aligned}
\bar{a} \delta & =\bar{a}(a d-b c)=(w c \bar{c}+1) d-(w \bar{c} d) c=d, \\
w \bar{c} \delta & =w \bar{c}(a d-b c)=(\bar{a} b) a-(a \bar{a}-1) b=b .
\end{aligned}
$$

This yields the bijection (3.5).

The defining equation

$$
|a(\zeta)|^{2}-1=w(\zeta) \cdot|c(\zeta)|^{2}
$$


for the pairs $(a(\zeta), c(\zeta)) \in \mathbb{Z}[\zeta]^{2}$ on the left hand side of $(3.5)$ is in the case $(a, c) \in \mathbb{Z}\left[p_{1}\right]^{2}$ and $w(\zeta) \in \mathbb{Z}\left[p_{1}\right]$ a Pell equation. We obtain the inequalities

$$
\begin{aligned}
0 & \leq|c(\zeta)|^{2}=w(\zeta)^{-1}\left(|a(\zeta)|^{2}-1\right) \\
|a(\zeta)| & \geq 1
\end{aligned}
$$

and

$$
\begin{aligned}
0 & \leq|c(\xi)|^{2}=(-w(\xi))^{-1}\left(1-|a(\xi)|^{2}\right)<(-w(\xi))^{-1} \\
|a(\xi)| & \leq 1 \text { for any primitive } m \text {-th unit root } \xi \notin\{\zeta, \bar{\zeta}\}
\end{aligned}
$$

$\Gamma$ maps $C$ to itself. Therefore by Poincaré's theorem, it is a Fuchsian group if it is a discrete matrix group. This holds if the set

$$
P_{1}:=\left\{a \in \mathbb{Z}[\zeta] \mid \exists c \in \mathbb{Z}[\zeta] \text { with }|a|^{2}-1=w \cdot|c|^{2}\right\}
$$

intersects each compact set $K \subset \mathbb{C}$ in a finite set.

The embedding $\underline{\sigma}: \mathbb{Q}(\zeta) \rightarrow \mathbb{R}^{\varphi(n)}$ from theorem 2.10 (a) maps $\mathbb{Z}[\zeta]$ to a lattice in $\mathbb{R}^{\varphi(n)}$. Because of $(3.10)$, it maps $P_{1} \cap K$ to a subset of

$$
\underline{\sigma}(\mathbb{Z}[\zeta]) \cap\left(K \times\{z \in \mathbb{C}|| z \mid \leq 1\}^{\varphi(n) / 2-1}\right) .
$$

This is a finite set. Therefore $\Gamma$ is a Fuchsian group.

The next lemma shows that the set $P_{1}$ and the group $\Gamma$ contain infinitely many elements.

Lemma 3.3. Let $m \in \mathbb{Z}_{\geq 3}, \zeta, p_{1}$ and $w \in \mathbb{Q}\left(p_{1}\right)$ be as in theorem 3.2. Then the set

$$
P_{2}:=\left\{(a, c) \in \mathbb{Z}\left[p_{1}\right] \mid a^{2}-1=w \cdot c^{2}\right\}
$$

contains infinitely many pairs. If $w \in \mathbb{Z}\left[p_{1}\right]$, then $P_{2}$ contains pairs $(a, c)$ with $w \mid(a-1)$.

Proof: If $\widetilde{w}=w \cdot u^{2}$ for some $u \in \mathbb{Z}\left[p_{1}\right]-\{0\}$ then a pair $(a, \widetilde{c}) \in$ $\mathbb{Z}\left[p_{1}\right]^{2}$ with $a^{2}-1=\widetilde{w} \cdot \widetilde{c}^{2}$ induces a pair $(a, c)=(a, \widetilde{c} \cdot u)$ in $P_{2}$. Therefore we can suppose $w \in \mathbb{Z}\left[p_{1}\right]$.

We will now construct infinitely many units in $\mathbb{Z}\left[\sqrt{w}, p_{1}\right]^{*}-\mathbb{Z}\left[p_{1}\right]^{*}$ and from them infinitely many pairs $(a, c)$ in $P_{2}$.

The algebraic number field $\mathbb{Q}\left(\sqrt{w}, p_{1}\right)$ has degree $\varphi(m)$ over $\mathbb{Q}$ and two real embeddings and $\varphi(m)-2$ complex embeddings, because of (3.1) and (3.2). By Dirichlet's unit theorem (theorem 2.10 (b)), the unit group $\mathbb{Z}\left[\sqrt{w}, p_{1}\right]^{*}$ of the order $\mathbb{Z}\left[\sqrt{w}, p_{1}\right]$ in $\mathbb{Q}\left(\sqrt{w}, p_{1}\right)$ contains a free abelian group of rank $2+\frac{\varphi(m)-2}{2}-1=\frac{\varphi(m)}{2}$.

The unit group $\mathbb{Z}\left[p_{1}\right]^{*}$ contains only a free abelian group of rank $\frac{\varphi(m)}{2}-1$. Therefore infinitely many units $a_{1}+\sqrt{w} c_{1} \in \mathbb{Z}\left[\sqrt{w}, p_{1}\right]^{*}$ with 
$a_{1} \neq 0$ and $c_{1} \neq 0$ exist. Then also $a_{1}-\sqrt{w} c_{1}$,

$$
\begin{aligned}
\left(a_{1}+\sqrt{w} c_{1}\right)^{2} & =\left(a_{1}^{2}+w c_{1}^{2}\right)+\sqrt{w}\left(2 a_{1} c_{1}\right)=: a_{2}+\sqrt{w} c_{2}, \\
\text { and } h & :=\left(a_{1}+\sqrt{w} c_{1}\right)\left(a_{1}-\sqrt{w} c_{1}\right)=a_{1}^{2}-w c_{1}^{2}
\end{aligned}
$$

are units, $h$ being in $\mathbb{Z}\left[p_{1}\right]^{*}$. Then

$$
\left(a_{3}, c_{3}\right):=\left(\frac{a_{2}}{h}, \frac{c_{2}}{h}\right) \in P_{2}
$$

because

$$
\begin{aligned}
a_{3}^{2}-w c_{3}^{2} & =h^{-2}\left(a_{2}^{2}-w c_{2}^{2}\right)=h^{-2}\left(a_{2}+\sqrt{w} c_{2}\right)\left(a_{2}-\sqrt{w} c_{2}\right) \\
& =h^{-2}\left(a_{1}+\sqrt{w} c_{1}\right)^{2}\left(a_{1}-\sqrt{w} c_{1}\right)^{2}=1 .
\end{aligned}
$$

Only finitely many units $a_{1}+\sqrt{w} c_{1}$ can give the same pair $\left(a_{3}, c_{3}\right)$. Therefore there are infinitely many pairs $\left(a_{3}, c_{3}\right)$ in $P_{2}$.

For the last statement, suppose that $\left(a_{4}, c_{4}\right) \in P_{2}$ with $c_{4} \neq 0$. Then the pair $\left(a_{5}, c_{5}\right):=\left(a_{4}^{2}+w c_{4}^{2}, 2 a_{4} c_{4}\right)$ is also in $P_{2}$,

$$
\begin{aligned}
a_{5}^{2}-w c_{5}^{2} & =\left(a_{5}+\sqrt{w} c_{5}\right)\left(a_{5}-\sqrt{w} c_{5}\right) \\
& =\left(a_{4}+\sqrt{w} c_{4}\right)^{2}\left(a_{4}-\sqrt{w} c_{4}\right)^{2}=\left(a_{4}-\sqrt{w} c_{4}\right)^{2}=1 .
\end{aligned}
$$

And it satisfies $w \mid\left(a_{5}-1\right)$ because of

$$
a_{5}-1=a_{4}^{2}+w c_{4}^{2}-1=2 w c_{4}^{2} .
$$

Remarks 3.4. (i) The equation $a^{2}-1=w c^{2}$ is for $w \in \mathbb{Z}\left[p_{1}\right]$ a Pell equation. A generalization of lemma 3.3 is theorem 3 in [Sch06].

(ii) The notion of an arithmetic Fuchsian group is defined in [Sh71, ch 9.2]. The group $\Gamma$ in theorem 3.2 is in fact an arithmetic Fuchsian group. This would follow immediately from [Ta75, theorem 2], if it were clear a priori that $\Gamma$ is a Fuchsian group of the first kind, i.e. a Fuchsian group with limit set $L(\Gamma)=C$. It follows with some work from a comparison of the data in theorem 3.2 with the data in Sh71, ch. 9.2].

(iii) The five triangle groups below in theorem 3.6 are arithmetic triangle groups. They are in the list in [Ta77, theorem 3] of all 85 arithmetic triangle groups.

(iv) Theorem 3.2 and lemma 3.3 will be used in the steps 2 and 4 in the proof of theorem 5.1 on the groups $G_{\mathbb{Z}}$ for the bimodal series.

Remarks 3.5. (i) The triangle groups below in theorem 3.6 will arise in theorem 6.1 as quotients of the groups $G_{\mathbb{Z}}$ for the quadrangle singularities. 
(ii) There the first six of the eight elements $w(\zeta)$ in table 5.72 in the case $r=0$ will be used. So here $W_{1,0}$ and $S_{1,0}$ are seen as 0 -th members of the series $W_{1, p}^{\sharp}$ and $S_{1, p}^{\sharp}$, not the series $W_{1, p}$ and $S_{1, p}$.

(iii) Using the notations and formulas from lemma 2.11, the first six of the eight elements $w(\zeta)$ in table $(5.72)$ in the case $r=0$ can be written as follows. In the case $U_{1,0}$ we change from $m=9$ to $m=18$, so below $\zeta=e^{2 \pi i / 18}$ for $E_{3,0}$ and $U_{1,0}$.

$$
\begin{aligned}
W_{1,0} \quad: \quad w(\zeta)=\frac{6}{\left(2-p_{1}\right) p_{1}}=\frac{1}{\left(2-p_{1}\right)\left(2+p_{1}\right)} \cdot 2 p_{1}\left(p_{1}+2\right) . \\
S_{1,0} \quad: \quad w(\zeta)=\frac{-2}{\left(-p_{3}\right)\left(-p_{3}-1\right)}=1 \cdot 2 p_{1}^{3} . \\
U_{1,0} \quad: \quad w(\zeta)=\frac{-3}{\left(2+p_{7}\right)\left(1-p_{1}\right)}=1 \cdot p_{1}\left(p_{1}+2\right) . \\
E_{3,0} \quad: \quad w(\zeta)=\frac{3\left(2-p_{1}\right)}{\left(p_{1}+2\right)\left(p_{1}-1\right)}=\left(2-p_{1}\right)^{2} \cdot p_{1}\left(p_{1}+2\right) . \\
Z_{1,0} \quad: \quad w(\zeta)=\frac{1}{-p_{5}}=1 \cdot\left(-p_{5}\right)^{-1}=1 \cdot\left(p_{1}-1\right) . \\
Q_{2,0} \quad: \quad w(\zeta)=\frac{2-p_{1}}{p_{1}+1}=\left(2-p_{1}\right) \cdot \frac{1}{p_{1}+1}
\end{aligned}
$$

(iv) In theorem 3.2 one can replace $w$ by $\widetilde{w}:=w \cdot u \bar{u}$ for any $u \in \mathbb{Z}[\zeta]^{*}$. The group $\Gamma$ for $w$ and the group $\widetilde{\Gamma}$ for $\widetilde{w}$ are isomorphic, and the triples in (3.5) are related by

$$
(\widetilde{a}, \widetilde{c}, \widetilde{\delta})=\left(a, c \cdot u^{-1}, \delta\right)
$$

We can choose $u$ such that $\widetilde{w}$ is simpler to work with than $w$. In the products for $w$ in (iii), the left terms are of the form $u \bar{u}$ for a suitable unit $u \in \mathbb{Z}[\zeta]^{*}$. The right terms are $\widetilde{w}$. We will work with the terms $\widetilde{w}$ in theorem 3.6 .

Theorem 3.6. The image in $P G L(2, \mathbb{C})$ of the group $\Gamma$ in theorem 3.2 for the following values of $m$ and $w$

$$
\begin{array}{l|l|l|l|l|l} 
& W_{1,0} & S_{1,0} & E_{3,0} \& U_{1,0} & Z_{1,0} & Q_{2,0} \\
m & 12 & 10 & 18 & 14 & 12 \\
w & 2 p_{1}\left(p_{1}+2\right) & 2 p_{1}^{3} & p_{1}\left(p_{1}+2\right) & \left(-p_{5}\right)^{-1} & \left(p_{1}+1\right)^{-1}
\end{array}
$$

is a Schwarzian triangle group of the following type:

$$
\begin{array}{l|l|l|l|l}
W_{1,0} & S_{1,0} & E_{3,0} \& U_{1,0} & Z_{1,0} & Q_{2,0} \\
(2,12,12) & (2,10,10) & (2,3,18) & (2,3,14) & (2,3,12)
\end{array}
$$


Proof: The proof has three steps. In step 1, we will present two matrices $A_{1}$ and $A_{2}$ in $\Gamma$ whose images in $P G L(2, \mathbb{C})$ are elliptic and generate in each case a Schwarzian triangle group of the claimed type. We will prove this. In step 2, we will show that no matrix in $\Gamma$ is closer to $A_{1}$ than $A_{2}$. This will be used in step 3 to prove that the images in $P G L(2, \mathbb{C})$ of $A_{1}$ and $A_{2}$ generate the image of $\Gamma$ in $P G L(2, \mathbb{C})$. The steps 1 and 3 together give theorem 3.6 .

Step 1: One checks easily with 3.5 that the following matrices $A_{1}$ and $A_{2}$ are in $\Gamma$.

$$
A_{1}=\left(\begin{array}{ll}
\zeta & 0 \\
0 & 1
\end{array}\right) \quad \text { for all } 5 \text { cases. }
$$

$$
\begin{aligned}
& W_{1,0}: \quad A_{2}=\left(\begin{array}{cc}
p_{1}+2 & -2 p_{1}\left(p_{1}+2\right) \\
1 & -\left(p_{1}+2\right)
\end{array}\right), \quad \operatorname{det} A_{2}=-1, \\
& S_{1,0}: \quad A_{2}=\left(\begin{array}{cc}
(\zeta+1) p_{1} & -2 p_{1}^{3} \zeta \\
1 & -(\zeta+1) p_{1}
\end{array}\right), \quad \operatorname{det} A_{2}=-\zeta \text {, } \\
& E_{3,0} \& U_{1,0}: \quad A_{2}=\left(\begin{array}{cc}
p_{1}+1 & -p_{1}\left(p_{1}+2\right) \\
1 & -\left(p_{1}+1\right)
\end{array}\right), \quad \operatorname{det} A_{2}=-1 \text {, } \\
& Z_{1,0}: \quad A_{2}=p_{1}\left(1-\zeta^{3}\right) \cdot\left(\begin{array}{cc}
1 & -\left(-p_{5}\right)^{-1} \\
1 & -1
\end{array}\right), \quad \operatorname{det} A_{2}=\zeta^{3}, \\
& Q_{2,0}: \quad A_{2}=\left(\begin{array}{cc}
\zeta+1 & -\zeta \\
p_{1}+1 & -(\zeta+1)
\end{array}\right), \quad \operatorname{det} A_{2}=-\zeta .
\end{aligned}
$$

A matrix $A \in G L(2, \mathbb{C})$ is elliptic if its eigenvalues $\lambda_{1}$ and $\lambda_{2}$ satisfy $\frac{\lambda_{2}}{\lambda_{1}} \in S^{1}$. Let $\left(\begin{array}{c}z_{j} \\ 1\end{array}\right)$ be an eigenvector with eigenvalue $\lambda_{j}$ for $j=1,2$ (possibly $z_{1}=0$ and $z_{2}=\infty$ ). Then the linear transformation of $A$ is a rotation around the fixed point $z_{1}$ with angle $\alpha(A)=\arg \frac{\lambda_{2}}{\lambda_{1}}$. For $A \in \Gamma$ elliptic we number the eigenvalues $\lambda_{1}, \lambda_{2}$ such that $\left|z_{1}\right|<\left|z_{2}\right|$, so then $\left|z_{1}\right|^{2}<w$ and $z_{1}$ is in the interior of the circle $C$. One sees in all 5 cases

$$
\begin{array}{r}
\lambda_{1}\left(A_{1}\right)=1, \quad \lambda_{2}\left(A_{1}\right)=\zeta, \quad \alpha\left(A_{1}\right)=\frac{2 \pi}{m}, \\
\operatorname{tr}\left(A_{2}\right)=0, \quad \alpha\left(A_{2}\right)=\pi .
\end{array}
$$


The following table lists for the product $A_{1} A_{2}$ the eigenvalues $\lambda_{1}, \lambda_{2}$ and the angle $\alpha_{1}\left(A_{3}\right)$.

$\begin{array}{llll} & \lambda_{1} & \lambda_{2} & \alpha \\ W_{1,0} & \zeta^{4} & \zeta^{3} & \frac{-2 \pi}{12} \\ S_{1,0} & \zeta^{4} & \zeta^{3} & \frac{-2 \pi}{10} \\ E_{3,0} \& U_{1,0} & \zeta^{8} & \zeta^{2} & \frac{-2 \pi}{3} \\ Z_{1,0} & e^{2 \pi i / 6} \zeta^{2} & e^{-2 \pi i / 6} \zeta^{2} & \frac{-2 \pi}{3} \\ Q_{2,0} & \zeta^{6} & \zeta^{2} & \frac{-2 \pi}{3}\end{array}$

Therefore the images of $A_{1}$ and $A_{2}$ in $P G L(2, \mathbb{C})$ generate a Schwarzian triangle group of the type in table (3.15) [Le64, VII 1G].

Step 2: Write $A_{2}=\left(\begin{array}{ll}a_{2} & b_{2} \\ c_{2} & d_{2}\end{array}\right)$ and write $A=\left(\begin{array}{ll}a & b \\ c & d\end{array}\right)$ for any $A \in \Gamma$.

Claim 1: Any $A \in \Gamma$ with $c \neq 0$ satisfies $|a| \geq\left|a_{2}\right|$.

The proof consists in making the proof of theorem 3.2 more constructive.

First we look for candidates $f \in \mathbb{Z}\left[p_{1}\right]$ of $|a|^{2}$ which are compatible with the inequalities (3.9) and (3.10) and which satisfy $f<\left|a_{2}\right|$. Then we will show that these candidates are not compatible with the equality $|a|^{2}=1+w \cdot|c|^{2}$.

Denote by $\underline{\sigma}^{\mathbb{R}}=\left(\sigma_{1}^{\mathbb{R}}, \ldots, \sigma_{\varphi(m) / 2}^{\mathbb{R}}\right): \mathbb{Q}\left(p_{1}\right) \rightarrow \mathbb{R}^{\varphi(m) / 2}$ the tuple of the embeddings $\sigma_{j}^{\mathbb{R}}: \mathbb{Q}\left(p_{1}\right) \rightarrow \mathbb{R}$. Then $\underline{\sigma}^{\mathbb{R}}\left(\mathbb{Z}\left[p_{1}\right]\right)$ is a $\mathbb{Z}$-lattice in $\mathbb{R}^{\varphi(m) / 2}$. The candidates are the numbers $f=f\left(p_{1}\right)$ in $\mathbb{Z}\left[p_{1}\right]$ with

$$
\left.\underline{\sigma}^{\mathbb{R}}(f) \in\right] 1,\left|a_{2}\right|^{2}[\times] 0,1\left[^{\varphi(m) / 2-1} .\right.
$$

This follows from the inequalities 3.9 and 3.10 . With sufficient numerical precision of the numbers $p_{j}$ in lemma 2.11, it is easy to find these candidates. They are as follows.

$$
\begin{array}{rlll}
W_{1,0} & : & f\left(p_{1}\right)=\alpha \cdot 1+\beta \cdot p_{1}, & (\alpha, \beta) \in\{(2,1),(4,2),(6,3)\} . \\
S_{1,0} & : & f\left(p_{1}\right)=\alpha \cdot 1+\beta \cdot p_{1}, & (\alpha, \beta) \in\{(2,2),(2,3)\} . \\
E_{3,0} & \& & U_{1,0}: \emptyset . \\
Z_{1,0} & : & \emptyset . \\
Q_{2,0} & : & \emptyset .
\end{array}
$$

All these candidates will be excluded with the help of the condition

$$
\operatorname{Norm}\left(|a|^{2}-1\right)=\operatorname{Norm}\left(w \cdot|c|^{2}\right)=\operatorname{Norm}(w) \cdot \operatorname{Norm}\left(|b|^{2}\right) .
$$

Here the norm is the norm in $\mathbb{Q}\left(p_{1}\right)$ and $\mathbb{Z}\left[p_{1}\right]$ with values in $\mathbb{Q}$ respectively $\mathbb{Z}$. 
The case $W_{1,0}: \operatorname{Norm}(w)=-12, \quad \operatorname{Norm}\left(1+p_{1}\right)=-2, \quad \operatorname{Norm}(3+$ $\left.2 p_{1}\right)=-3, \quad \operatorname{Norm}\left(5+3 p_{1}\right)=-2$.

The case $S_{1,0}: \operatorname{Norm}(w)=-4, \quad \operatorname{Norm}\left(1+2 p_{1}\right)=-1, \quad \operatorname{Norm}(1+$ $\left.3 p_{1}\right)=-5$.

Step 3: It is sufficient to show the following claim 2.

Claim 2: For any matrix $A_{3} \in \Gamma$ with $c_{3} \neq 0$, a number $k \in \mathbb{Z}$ exists such that the product

$$
A_{4}:=A_{3} \cdot A_{1}^{-k} A_{2} A_{1}^{k}=\left(\begin{array}{cc}
a_{3} & b_{3} \\
c_{3} & d_{3}
\end{array}\right)\left(\begin{array}{cc}
a_{2} & \zeta^{-k} b_{2} \\
\zeta^{k} c_{2} & d_{2}
\end{array}\right)
$$

satisfies

$$
\left|c_{4}\right|<\left|c_{3}\right|, \quad \text { here } c_{4}=c_{3} a_{2}+\zeta^{k} d_{3} c_{2}
$$

We can choose $k \in \mathbb{Z}$ such that

$$
\beta:=\left|\arg \left(c_{3} a_{2}\right)-\arg \left(-\zeta^{k} d_{3} c_{2}\right)\right| \leq \frac{\pi}{m} .
$$

Observe

$$
\frac{\left|\zeta^{k} d_{3} c_{2}\right|^{2}}{\left|c_{3} a_{2}\right|^{2}}=\frac{\left|a_{3}\right|^{2} \frac{\left|a_{2}\right|^{2}-1}{w(\zeta)}}{\frac{\left|a_{3}\right|^{2}-1}{w(\zeta)}\left|a_{2}\right|^{2}}=\frac{1-\left|a_{2}\right|^{-2}}{1-\left|a_{3}\right|^{-2}} .
$$

The trivial inequality $1-\left|a_{3}\right|^{-2}<1$ and the inequality $\left|a_{3}\right| \geq\left|a_{2}\right|$ from step 2 give the inequalities

$$
\left(1-\left|a_{2}\right|^{-2}\right)\left|c_{3} a_{2}\right|^{2}<\left|\zeta^{k} d_{3} c_{2}\right|^{2} \leq\left|c_{3} a_{2}\right|^{2} .
$$

Observe also

$$
\sqrt{1-\left|a_{2}\right|^{-2}}<\cos \frac{\pi}{m}
$$

Therefore

$$
\begin{aligned}
\left|c_{4}\right| & =\left|c_{3} a_{2}\right|^{2}(\sin \beta)^{2}+\left(\left|c_{3} a_{2}\right| \cos \beta-\left|d_{3} c_{2}\right|\right)^{2} \\
& <\left|c_{3} a_{2}\right|^{2}\left(\sin \frac{\pi}{m}\right)^{2}+\left(1-\sqrt{1-\left|a_{2}\right|^{-2}}\right)^{2} \cdot\left|c_{3} a_{2}\right|^{2} \\
& \left.=\left|c_{3}\right|^{2} \cdot\left|a_{2}\right|^{2}\left(\left(\sin \frac{\pi}{m}\right)^{2}+\left(1-\sqrt{1-\left|a_{2}\right|^{-2}}\right)\right)^{2}\right) \\
& \stackrel{(*)}{<}\left|c_{3}\right|^{2} .
\end{aligned}
$$

$\stackrel{(*)}{<}$ follows in all 5 cases by an explicit calculation. 


\section{REVIEW ON THE TOPOLOGY OF SINGULARITIES}

In this section, we recall some classical facts about the topology of singularities, and we fix some notations.

An isolated hypersurface singularity (short: singularity) is a holomorphic function germ $f:\left(\mathbb{C}^{n+1}, 0\right) \rightarrow(\mathbb{C}, 0)$ with an isolated singularity at 0 . Its Jacobi ideal is

$$
J(f):=\left(\frac{\partial f}{\partial x_{0}}, \ldots, \frac{\partial f}{\partial x_{n}}\right) \subset \mathcal{O}_{\mathbb{C}^{n+1}, 0}
$$

Its Jacobi algebra is $\mathcal{O}_{\mathbb{C}^{n+1}, 0} / J(f)$. Its Milnor number $\mu:=$ $\operatorname{dim} \mathcal{O}_{\mathbb{C}^{n+1}, 0} / J(f)$ is finite. For the following notions and facts compare [AGV88, and [Eb07. A good representative of $f$ has to be defined with some care [Mi68] AGV88] Eb07]. It is $f: X \rightarrow \Delta$ with $\Delta=\{\tau \in \mathbb{C}|| \tau \mid<\delta\}$ a small disk around 0 and $X=\left\{x \in \mathbb{C}^{n+1}|| x \mid<\right.$ $\varepsilon\} \cap f^{-1}(\Delta)$ for some sufficiently small $\varepsilon>0$ (first choose $\varepsilon$, then $\delta$ ). Then $f: X^{\prime} \rightarrow \Delta^{\prime}$ with $X^{\prime}=X-f^{-1}(0)$ and $\Delta^{\prime}=\Delta-\{0\}$ is a locally trivial $C^{\infty}$-fibration, the Milnor fibration. Each fiber has the homotopy type of a bouquet of $\mu n$-spheres [Mi68.

Therefore the (reduced for $n=0$ ) middle homology groups are $H_{n}^{(r e d)}\left(f^{-1}(\tau), \mathbb{Z}\right) \cong \mathbb{Z}^{\mu}$ for $\tau \in \Delta^{\prime}$. Each comes equipped with an intersection form $I$, which is a datum of one fiber, a monodromy $M_{h}$ and a Seifert form $L$, which come from the Milnor fibration, see AGV88, I.2.3] for their definitions. $M_{h}$ is a quasiunipotent automorphism, $I$ and $L$ are bilinear forms with values in $\mathbb{Z}, I$ is $(-1)^{n}$-symmetric, and $L$ is unimodular. $L$ determines $M_{h}$ and $I$ because of the formulas AGV88, I.2.3]

$$
\begin{aligned}
L\left(M_{h} a, b\right) & =(-1)^{n+1} L(b, a) \\
I(a, b) & =-L(a, b)+(-1)^{n+1} L(b, a)=L((M-\mathrm{id}) a, b)
\end{aligned}
$$

(4.2) tells especially that $\operatorname{ker}\left(M_{h}-\mathrm{id}\right)$ is the radical of $I$ and that $L$ is $(-1)^{n+1}$-symmetric on this radical. The semisimple part of $M_{h}$ is called $M_{s}$, the unipotent part $M_{u}$, the nilpotent part $N=\log M_{u}$.

The Milnor lattices $H_{n}\left(f^{-1}(\tau), \mathbb{Z}\right)$ for all Milnor fibrations $f: X^{\prime} \rightarrow$ $\Delta^{\prime}$ and then all $\tau \in \mathbb{R}_{>0} \cap T^{\prime}$ are canonically isomorphic, and the isomorphisms respect $M_{h}, I$ and $L$. This follows from Lemma 2.2 in [LR73]. These lattices are identified and called Milnor lattice $M l(f)$.

The group $G_{\mathbb{Z}}$ is

$$
G_{\mathbb{Z}}=G_{\mathbb{Z}}(f):=\operatorname{Aut}(M l(f), L)=\operatorname{Aut}\left(M l(f), M_{h}, I, L\right),
$$


the second equality is true because $L$ determines $M_{h}$ and $I$. A good control of this group for the bimodal series and the quadrangle singularities will be crucial in this paper. It is the task of the sections 5 and 6.

The Milnor lattice comes equipped with a set $\mathcal{B}$ of distinguished bases, certain tuples $\underline{\delta}=\left(\delta_{1}, \ldots, \delta_{\mu}\right)$ of $\mathbb{Z}$-bases of the Milnor lattice. Each one is defined with a generic deformation of $f$ which has $\mu A_{1}$ singularities which have all different critical values. One chooses a distinguished system of paths in $\Delta$ from the critical values to $\delta \in \partial \Delta$ and pushes vanishing cycles along these paths to $H_{n}\left(f^{-1}(\delta), \mathbb{Z}\right)=M l(f)$. See [AGV88] or [Eb07] for details. In all cases except the simple singularities, the set $\mathcal{B}$ is infinite. Each distinguished basis determines the monodromy by the formula

$$
M_{h}=s_{\delta_{1}} \circ \ldots \circ s_{\delta_{\mu}}
$$

where

$$
\begin{aligned}
s_{\delta} & : \quad M l(f) \rightarrow M l(f), \\
s_{\delta}(b) & :=\quad b-(-1)^{n(n+1) / 2} \cdot I(\delta, b) \cdot \delta,
\end{aligned}
$$

is the Picard-Lefschetz transformation of a vanishing cycle $\delta$, a reflection for even $n$ and a symplectic transvection for odd $n$.

The matrix of the Seifert form with respect to a distinguished basis is lower triangular with $(-1)^{(n+1)(n+2) / 2}$ on the diagonal. This motivates two definitions, the normalized Seifert form

$$
L^{\text {hnor }}:=(-1)^{(n+1)(n+2) / 2} \cdot L,
$$

and the Stokes matrix $S$ of the distinguished basis with

$$
S:=(-1)^{(n+1)(n+2) / 2} \cdot L\left(\underline{\delta}^{t}, \underline{\delta}\right)^{t}=L^{h n o r}\left(\underline{\delta}^{t}, \underline{\delta}\right)^{t} .
$$

$S$ is an upper triangular matrix in $G L(\mu, \mathbb{Z})$ with 1 's on the diagonal.

The Coxeter-Dynkin diagram of a distinguished basis encodes $S$ in a geometric way. It has $\mu$ vertices which are numbered from 1 to $\mu$. Between two vertices $i$ and $j$ with $i<j$ one draws

$$
\begin{array}{ll}
\text { no edge } & \text { if } S_{i j}=0, \\
\left|S_{i j}\right| \text { edges } & \text { if } S_{i j}<0, \\
S_{i j} \text { dotted edges } & \text { if } S_{i j}>0
\end{array}
$$

Coxeter-Dynkin diagrams for the 8 bimodal series will be given in section 5, following Eb81.

A result of Thom and Sebastiani compares the Milnor lattices and monodromies of the singularities $f=f\left(x_{0}, \ldots, x_{n}\right), g=g\left(y_{0}, \ldots, y_{m}\right)$ and $f+g=f\left(x_{0}, \ldots, x_{n}\right)+g\left(x_{n+1}, \ldots, x_{m+n+1}\right)$. There are extensions by Deligne for the Seifert form and by Gabrielov for distinguished bases. 
All results are in AGV88, I.2.7]. They are restated here. There is a canonical isomorphism

$$
\begin{aligned}
\Phi: M l(f+g) & \cong M l(f) \otimes M l(g), \\
\text { with } M_{h}(f+g) & \cong M_{h}(f) \otimes M_{h}(g) \\
\text { and } L^{\text {hnor }}(f+g) & \cong L^{\text {hnor }}(f) \otimes L^{\text {hnor }}(g) .
\end{aligned}
$$

If $\underline{\delta}=\left(\delta_{1}, \ldots, \delta_{\mu(f)}\right)$ and $\underline{\gamma}=\left(\gamma_{1}, \ldots, \gamma_{\mu(g)}\right)$ are distinguished bases of $f$ and $g$ with Stokes matrices $S(f)$ and $S(g)$, then

$\Phi^{-1}\left(\delta_{1} \otimes \gamma_{1}, \ldots, \delta_{1} \otimes \gamma_{\mu(g)}, \delta_{2} \otimes \gamma_{1}, \ldots, \delta_{2} \otimes \gamma_{\mu(g)}, \ldots, \delta_{\mu(f)} \otimes \gamma_{1}, \ldots, \delta_{\mu(f)} \otimes \gamma_{\mu(g)}\right)$

is a distinguished basis of $M l(f+g)$, that means, one takes the vanishing cycles $\Phi^{-1}\left(\delta_{i} \otimes \gamma_{j}\right)$ in the lexicographic order. Then by (4.7) and (4.10), the matrix

$$
S(f+g)=S(f) \otimes S(g)
$$

(where the tensor product is defined so that it fits to the lexicographic order) is the Stokes matrix of this distinguished basis.

In the special case $g=x_{n+1}^{2}$, the function germ $f+g=$ $f\left(x_{0}, \ldots, x_{n}\right)+x_{n+1}^{2} \in \mathcal{O}_{\mathbb{C}^{n+2}, 0}$ is called stabilization or suspension of $f$. As there are only two isomorphisms $\operatorname{Ml}\left(x_{n+1}^{2}\right) \rightarrow \mathbb{Z}$, and they differ by a sign, there are two equally canonical isomorphisms $M l(f) \rightarrow M l\left(f+x_{n+1}^{2}\right)$, and they differ just by a sign. Therefore automorphisms and bilinear forms on $M l(f)$ can be identified with automorphisms and bilinear forms on $M l\left(f+x_{n+1}^{2}\right)$. In this sense AGV88, I.2.7]

$$
\begin{aligned}
L^{\text {hnor }}\left(f+x_{n+1}^{2}\right) & =L^{\text {hnor }}(f), \\
M\left(f+x_{n+1}^{2}\right) & =-M(f), \\
G_{\mathbb{Z}}\left(f+x_{n+1}^{2}\right) & =G_{\mathbb{Z}}(f) .
\end{aligned}
$$

The image in $M l\left(f+x_{n+1}^{2}\right)$ of a distinguished basis in $M l(f)$ under either of the both isomorphisms $M l(f) \rightarrow M l\left(f+x_{n+1}^{2}\right)$ is again a distinguished basis, and it has the same Stokes matrix.

Denote by $H_{\mathbb{C}}^{\infty}$ the $\mu$-dimensional vector space of global flat multivalued sections in the flat cohomology bundle $\bigcup_{\tau \in \Delta^{\prime}} H^{n}\left(f^{-1}(\tau), \mathbb{C}\right)$ (reduced cohomology for $n=0)$. It comes equipped with a $\mathbb{Z}$-lattice $H_{\mathbb{Z}}^{\infty}$, a real subspace $H_{\mathbb{R}}^{\infty}$, a monodromy which is also denoted by $M_{h}$, and the dual $L^{\text {nor }}$ of the normalized Seifert form $L^{\text {hnor }}$. It is a unimodular form on $H_{\mathbb{Z}}^{\infty}$, and the analogue of 4.1,

$$
L^{\text {nor }}\left(M_{h} a, b\right)=(-1)^{n+1} L^{n o r}(b, a) \quad \text { for } a, b \in H_{\mathbb{Z}}^{\infty},
$$

holds. 
We apply the notations 2.1 (a) to $M l(f)$ and to $H_{\mathbb{Z}}^{\infty}$ and extend them slightly:

$$
\begin{aligned}
M l(f)_{\lambda} & :=\operatorname{ker}\left(M_{h}-\lambda \mathrm{id}\right)^{\mu}: M l(f)_{\mathbb{C}} \rightarrow M l(f)_{\mathbb{C}} \\
M l(f)_{\neq 1} & :=\bigoplus_{\lambda \neq 1} M l(f)_{\lambda}, \quad M l(f)_{\neq-1}:=\bigoplus_{\lambda \neq-1} M l(f)_{\lambda}, \\
M l(f)_{p} & :=\bigoplus_{\lambda: p(\lambda)=0} M l(f)_{\lambda}, \quad M l(f)_{p, \mathbb{Z}}:=M l(f)_{p} \cap M l(f) .
\end{aligned}
$$

$H_{\lambda}^{\infty}, H_{\neq 1}^{\infty}, H_{\neq-1}^{\infty}, H_{p}^{\infty}$ and $H_{p, \mathbb{Z}}^{\infty}$ are defined analogously.

There are a natural Hodge filtration $F_{S t}^{\bullet}$ on $H_{\mathbb{C}}^{\infty}$ and a weight filtration $W_{\bullet}$ on $H_{\mathbb{Q}}^{\infty}$ such that $\left(H_{\neq 1}^{\infty}, H_{\neq 1, \mathbb{Z}}^{\infty}, F_{S t}^{\bullet}, W_{\bullet},-N, S\right)$ is a polarized mixed Hodge structure of weight $n$ and $\left(H_{1}^{\infty}, H_{1, \mathbb{Z}}^{\infty}, F_{S t}^{\bullet}, W_{\bullet},-N, S\right)$ is a polarized mixed Hodge structure of weight $n+1$ [He02, Theorem $10.30]$.

In the case of a singularity with semisimple monodromy, so $N=0$, the weight filtrations become trivial, and the polarized mixed Hodge structures are polarized pure Hodge structures. This holds for all bimodal singularities. Therefore we do not care here about the weight filtration. We will define the Hodge filtration using the Brieskorn lattice in theorem 7.7 (following Varchenko, Scherk\&Steenbrink and M. Saito).

The pure Hodge structure of weight $n$ on $H_{\neq 1}^{\infty}$ for any singularity with semisimple monodromy has the following properties. The Hodge filtration is $M_{s}$-invariant and satisfies

$$
H_{\lambda}^{\infty}=\bigoplus_{p \in \mathbb{Z}} H_{\lambda}^{p, n-p} \text { for } \lambda \neq 1
$$

where $H_{\lambda}^{p, n-p}:=\left(F^{p} \cap \overline{F^{n-p}}\right) H_{\lambda}^{\infty}, \quad\left(\Rightarrow H_{\bar{\lambda}}^{n-p, p}=\overline{H_{\lambda}^{p, n-p}},\right)$

equivalently $H_{\lambda}^{\infty}=F^{p} H_{\lambda}^{\infty} \oplus \overline{F^{n+1-p} H_{\lambda}^{\infty}}$.

The polarizing form carries an isotropy and a positivity condition,

$$
\begin{aligned}
& S\left(H_{\lambda}^{p, n-p}, H_{\bar{\lambda}}^{q, n-q}\right)=0 \quad \text { if } p+q \neq 0, \\
& i^{p-(n-p)} \cdot S(a, \bar{a})>0 \quad \text { for } a \in H_{\lambda}^{p, n-p}-\{0\} .
\end{aligned}
$$

The pure Hodge structure of weight $n+1$ on $H_{1}^{\infty}$ has analogous properties, with $n$ replaced by $n+1$.

The polarizing form $S: H_{\mathbb{Q}}^{\infty} \times H_{\mathbb{Q}}^{\infty} \rightarrow \mathbb{Q}$ is defined by [He02, 10.6].

$$
S(a, b):=-L^{n o r}(a, \nu b)
$$


where $\nu: H_{\mathbb{Q}}^{\infty} \rightarrow H_{\mathbb{Q}}^{\infty}$ is the $M_{h}$-invariant automorphism

$$
\nu:= \begin{cases}\frac{1}{M_{h}-\mathrm{id}} & \text { on } H_{\neq 1}^{\infty}, \\ \frac{-N}{M_{h}-\mathrm{id}} & \text { on } H_{1}^{\infty},\end{cases}
$$

$S$ is nondegenerate and $M_{h}$-invariant. It is $(-1)^{n}$-symmetric on $H_{\neq 1}^{\infty}$ and $(-1)^{n+1}$-symmetric on $H_{1}^{\infty}$. The restriction to $H_{\neq 1}^{\infty}$ is $(-1)^{n(n+1) / 2}$. $I^{\vee}$, where $I^{\vee}$ on $H_{\neq 1}^{\infty}$ is dual to $I$ (which is nondegenerate on $M l(f)_{\neq 1}$ ).

\section{The Group $G_{\mathbb{Z}}$ FOR The BIMOdal SERIES SINGUlarities}

The normal forms from [AGV85, §13] for the eight bimodal series will be listed below in section 9. The following table gives their names, the Milnor numbers, certain polynomials $b_{1}, b_{2}$ or, in the case of the series $Z_{1, p}$, polynomials $b_{1}, b_{2}, b_{3}$ such that $b_{1} b_{2}$ respectively $b_{1} b_{2} b_{3}$ is the characteristic polynomial of the surface singularities, and two important numbers $m$ and $r_{I}$. In the series $p \in \mathbb{Z}_{\geq 1}$.

\begin{tabular}{lllllll} 
series & $\mu$ & $b_{1}$ & $b_{2}$ & $b_{3}$ & $m$ & $r_{I}$ \\
\hline$W_{1, p}^{\sharp}$ & $15+p$ & $\Phi_{12}$ & $\left(t^{12+p}-1\right) / \Phi_{1}$ & - & 12 & 1 \\
$S_{1, p}^{\sharp}$ & $14+p$ & $\Phi_{10} \Phi_{2}$ & $\left(t^{10+p}-1\right) / \Phi_{1}$ & - & 10 & 1 \\
$U_{1, p}$ & $14+p$ & $\Phi_{9}$ & $\left(t^{9+p}-1\right) / \Phi_{1}$ & - & 9 & 1 \\
$E_{3, p}$ & $16+p$ & $\Phi_{18} \Phi_{2}$ & $t^{9+p}+1$ & - & 18 & 2 \\
$Z_{1, p}$ & $15+p$ & $\Phi_{14} \Phi_{2}$ & $t^{7+p}+1$ & $\Phi_{2}$ & 14 & 2 \\
$Q_{2, p}$ & $14+p$ & $\Phi_{12} \Phi_{4} \Phi_{3}$ & $t^{6+p}+1$ & - & 12 & 2 \\
$W_{1, p}$ & $15+p$ & $\Phi_{12} \Phi_{6} \Phi_{3} \Phi_{2}$ & $t^{6+p}+1$ & - & 12 & 2 \\
$S_{1, p}$ & $14+p$ & $\Phi_{10} \Phi_{5} \Phi_{2}$ & $t^{5+p}+1$ & - & 10 & 2
\end{tabular}

The following theorem on the group $G_{\mathbb{Z}}$ will be proved in two steps. Directly after the theorem, the arguments and properties which hold for all eight series will be given. Then in eight subsections, one for each series, the corresponding objects will be made explicit and some specific details will be given. For each series, denote $\zeta:=e^{2 \pi i / m} \in S^{1} \subset \mathbb{C}$.

Theorem 5.1. For any surface singularity $f$ in any of the eight bimodal series, the following holds.

(a) (See definition 2.3 for the notion Orlik block) For all series except $Z_{1, p}$, there are Orlik blocks $B_{1}, B_{2} \subset M l(f)$, and for the series $Z_{1, p}$, there are Orlik blocks $B_{1}, B_{2}, B_{3} \subset M l(f)$ with the following properties. The characteristic polynomial $p_{B_{j}}$ of the monodromy on $B_{j}$ is $b_{j}$. The sum $\sum_{j \geq 1} B_{j}$ is a direct sum $\bigoplus_{j \geq 1} B_{j}$, and it is a sublattice of $M l(f)$ of full rank $\mu$ and of index $r_{I}$. Define

$$
\widetilde{B}_{1}:= \begin{cases}B_{1} & \text { for all series except } Z_{1, p} \\ B_{1} \oplus B_{3} & \text { for the series } Z_{1, p} .\end{cases}
$$


Then

$$
\begin{aligned}
L\left(\widetilde{B}_{1}, B_{2}\right) & =0=L\left(B_{2}, \widetilde{B}_{1}\right) \quad \text { for all series, } \\
G_{\mathbb{Z}} & =\operatorname{Aut}\left(\bigoplus_{j \geq 1} B_{j}, L\right) \quad \text { for all series except } S_{1,10}
\end{aligned}
$$

In the case $S_{1,10}$, a substitute for (5.4) is

$g \in G_{\mathbb{Z}}$ with $g\left(\left(B_{1}\right)_{\Phi_{10}}\right)=\left(B_{1}\right)_{\Phi_{10}} \Rightarrow g\left(B_{j}\right)=B_{j}$ for $j=1,2$.

(b) $\Phi_{m} \backslash b_{2} \Longleftrightarrow m \backslash$ p. In that case

$$
G_{\mathbb{Z}}=\left\{\left( \pm\left. M_{h}^{k_{1}}\right|_{\widetilde{B}_{1}}\right) \times\left( \pm\left. M_{h}^{k_{2}}\right|_{B_{2}}\right) \mid k_{1}, k_{2} \in \mathbb{Z}\right\} .
$$

(c) In the case of the subseries with $m \mid p$, the eigenspace $\operatorname{Ml}(f)_{\zeta} \subset$ $M l(f)_{\mathbb{C}}$ is 2-dimensional. The hermitian form $h_{\zeta}$ on it from lemma 2.2 (a) with $h_{\zeta}(a, b):=\sqrt{-\zeta} \cdot L(a, \bar{b})$ for $a, b \in M l(f)_{\zeta}$ is nondegenerate and indefinite, so $\mathbb{P}\left(M l(f)_{\zeta}\right) \cong \mathbb{P}^{1}$ contains a half-plane

$$
\mathcal{H}_{\zeta}:=\left\{\mathbb{C} \cdot a \mid a \in M l(f)_{\zeta} \text { with } h_{\zeta}(a, a)<0\right\} \subset \mathbb{P}\left(M l(f)_{\zeta}\right) .
$$

Therefore the group $\operatorname{Aut}\left(M l(f)_{\zeta}, h_{\zeta}\right) / S^{1} \cdot \mathrm{id}$ is isomorphic to $P S L_{2}(\mathbb{R})$. The homomorphism

$$
\Psi: G_{\mathbb{Z}} \rightarrow \operatorname{Aut}\left(M l(f)_{\zeta}, h_{\zeta}\right) / S^{1} \cdot \mathrm{id},\left.\quad g \mapsto g\right|_{M l(f)_{\zeta}} \bmod S^{1} \cdot \mathrm{id},
$$

is well-defined. $\Psi\left(G_{\mathbb{Z}}\right)$ is an infinite Fuchsian group acting on the halfplane $\mathcal{H}_{\zeta}$. And

$$
\operatorname{ker} \Psi=\left\{ \pm M_{h}^{k} \mid k \in \mathbb{Z}\right\} .
$$

Proof: Here we explain the common arguments of the proof, which hold for all eight series. We will announce definitions and properties of several objects. In the following eight subsections, one for each series, the objects will be defined, and their properties will be shown.

(a) For each of the eight series of surface singularities, a distinguished basis $e_{1}, \ldots, e_{\mu}$ with the Coxeter-Dynkin diagram in the corresponding figure will be given in the subsections 5.1 to 5.8 . The distinguished basis is the one in [Eb81, Tabelle $6 \&$ Abb. 16], with a small change in the cases $W_{1,1}$ and $S_{1,1}$. They are exceptional in [Eb81. With the actions of the braids $\alpha_{1}, \ldots, \alpha_{\mu-1}$ (see [Eb07, 5.7] for these braids and their actions) and a sign change, we arrive at a new numbering of the same unnumbered diagram, such that $W_{1,1}$ and $S_{1,1}$ are no longer exceptional (i.e. the top vertex has the number $p+q+r+3$ in the notation of [Eb81, Abb. 16] even for $W_{1,1}$ and $\left.S_{1,1}\right)$. We thank Wolfgang Ebeling for the explanation how to arrive at this numbering. 
Recall that for a surface singularity (then $n=2$ ) the reflection along a vanishing cycle $\delta$ is

$$
s_{\delta}(b)=b+I(\delta, b) \cdot \delta \quad \text { for any } b \in M l(f) .
$$

The Coxeter-Dynkin diagram has between the vertices $i$ and $j$ with $i<j$ no edge if $S_{i j}=0,\left|S_{i j}\right|$ edges if $S_{i j}<0$ and $S_{i j}$ dotted edges if $S_{i j}>0$. Here for $i<j$

$$
\begin{array}{lll}
I\left(e_{i}, e_{j}\right)=I\left(e_{j}, e_{i}\right)=-S_{i j}, & I\left(e_{i}, e_{i}\right)=-2 \\
L\left(e_{i}, e_{j}\right)=0, & L\left(e_{j}, e_{i}\right)=S_{i j}, & L\left(e_{i}, e_{i}\right)=1
\end{array}
$$

The monodromy can be calculated fairly efficiently by hand (one should write down some intermediate steps) with the formula

$$
M_{h}=s_{e_{1}} \circ \ldots \circ s_{e_{\mu}} .
$$

The cyclic sublattices $B_{j} \subset M l(f)$ are chosen by choosing the generating lattice vectors $\beta_{j}$ with

$$
B_{j}:=\sum_{i \geq 0} \mathbb{Z} \cdot M_{h}^{i}\left(\beta_{j}\right)
$$

The following table gives them.

\begin{tabular}{llll} 
series & $\beta_{1}$ & $\beta_{2}$ & $\beta_{3}$ \\
\hline$W_{1, p}^{\sharp}$ & $e_{3}$ & $e_{8}$ & - \\
$S_{1, p}^{\sharp}$ & $e_{8}$ & $e_{9}$ & - \\
$U_{1, p}$ & $e_{8}$ & $e_{10}-$ \\
$E_{3, p}$ & $e_{3}$ & $e_{10}-$ \\
$Z_{1, p}$ & $e_{8}$ & $e_{11}$ & $e_{3}-e_{4}-e_{9}$ \\
$Q_{2, p}$ & $e_{8}$ & $e_{11}-$ \\
$W_{1, p}$ & $e_{3}+e_{9}+e_{11}$ & $e_{16}-$ \\
$S_{1, p}$ & $-e_{8}+e_{13}$ & $e_{15}-$
\end{tabular}

We will write down the action of the powers of the monodromy,

$$
\beta_{j} \mapsto M_{h}\left(\beta_{j}\right) \mapsto M_{h}^{2}\left(\beta_{j}\right) \mapsto \ldots \mapsto M_{h}^{\operatorname{deg} b_{j}}\left(\beta_{j}\right),
$$

in the subsections. Verifying $b_{j}\left(M_{h}\right)\left(\beta_{j}\right)=0$ will show that the characteristic polynomial of $M_{h}$ on $B_{j}$ is $b_{j}$. We will also write down nice generators of $B_{j}$. This will show that $B_{j}$ is a primitive sublattice of $M l(f)$, that $\sum_{j \geq 1} B_{j}=\bigoplus_{j \geq 1} B_{j}$ is a direct sum and that it is a sublattice of full rank and of index $r_{I}$ in $M l(f)$. In all cases except $W_{1, p}$ and $S_{1, p}$, the index $r_{I}$ is obvious from the nice generators, in the two cases $W_{1, p}$ and $S_{1, p}$, it requires the calculation of a determinant.

The left and right $L$-orthogonality of $\widetilde{B}_{1}$ and $B_{2}$ in $(5.3)$ will be proved now. $e_{\mu}$ is a cyclic generator for $B_{2}$ in all eight series. The 
nice generators for $\widetilde{B}_{1}$ show $\widetilde{B}_{1} \subset \bigoplus_{j=1}^{\mu-2} \mathbb{Z} \cdot e_{j}$ for all cases except $W_{1,1}$ and $S_{1,1}$. This and $L\left(e_{i}, e_{\mu}\right)=0$ for $i<\mu$ show $L\left(\widetilde{B}_{1}, e_{\mu}\right)=0$, thus $L\left(\widetilde{B}_{1}, B_{2}\right)=0$. From the CDD one sees easily $L\left(e_{\mu}, e_{i}\right)=0$ for $i \leq \mu-2$ for all cases except $W_{1,1}$ and $S_{1,1}$, thus $L\left(e_{\mu}, \widetilde{B}_{1}\right)=0$ and $L\left(B_{2}, \widetilde{B}_{1}\right)=0$. For the cases $W_{1,1}$ and $S_{1,1}, L\left(B_{1}, e_{\mu}\right)=0=L\left(e_{\mu}, B_{1}\right)$ and thus $L\left(B_{1}, B_{2}\right)=0=L\left(B_{2}, B_{1}\right)$ hold also.

(5.5) for $S_{1,10}$ will be shown in subsection 5.8. With respect to part (a), it rests to show (5.4). It is trivial for the 3 series with $r_{I}=1$. It will be shown in subsection 5.6 for the series $Q_{2, p}$ and in subsection 5.7 for the subseries $W_{1,6 s-3}\left(s \in \mathbb{Z}_{\geq 1}\right)$ of the series $W_{1, p}$. For all other series, it will be shown below. It requires a study of smaller Orlik blocks. $\Phi_{2} \mid b_{1}$ holds in the series $S_{1, p}^{\sharp}, E_{3, p}, Z_{1, p}, W_{1, p}$ and $S_{1, p}$. In these cases define (see 2.16) for the notion $v\left(\beta_{1},-1\right)$ )

$$
\gamma_{1}:=v\left(\beta_{1},-1\right):=\frac{b_{1}}{\Phi_{2}}\left(M_{h}\right)\left(\beta_{1}\right)
$$

and calculate $L\left(\gamma_{1}, \gamma_{1}\right)$ using (2.17): $L\left(\gamma_{1}, \gamma_{1}\right)=\frac{b_{1}}{\Phi_{2}}(-1) \cdot L\left(\gamma_{1}, \beta_{1}\right)$.

$$
\begin{array}{lll}
\text { series } & \gamma_{1} & L\left(\gamma_{1}, \gamma_{1}\right) \\
\hline S_{1, p}^{\sharp} & \Phi_{10}\left(M_{h}\right)\left(e_{8}\right)=2 e_{1}+e_{2}-e_{4}-e_{5}-e_{6}+e_{8} & 5 \\
E_{3, p} & \Phi_{18}\left(M_{h}\right)\left(e_{3}\right)=-e_{2}+2 e_{3}+e_{6}-e_{7}+e_{9} & 6 \\
Z_{1, p} & \Phi_{14}\left(M_{h}\right)\left(e_{8}\right) & \\
& =e_{2}+e_{3}-3 e_{4}-e_{6}+e_{7}-3 e_{9}-e_{10} & 21 \\
W_{1, p} & \left(\Phi_{12} \Phi_{6} \Phi_{3}\right)\left(M_{h}\right)\left(e_{3}+e_{9}+e_{11}\right) & \\
& =e_{4}-e_{5}+e_{9}+e_{11}-e_{13}-e_{15} & 6 \\
S_{1, p} & \left(\Phi_{10} \Phi_{5}\right)\left(M_{h}\right)\left(-e_{8}+e_{13}\right) & \\
& =-2 e_{1}+e_{7}-e_{8}-e_{9}-e_{11}-e_{12}-e_{14} & 10
\end{array}
$$

In the case of the series $Z_{1, p}$, define $\gamma_{3}:=\beta_{3}$ and calculate

$$
L\left(\gamma_{3}, \gamma_{3}\right)=3, \quad L\left(\gamma_{1}, \gamma_{3}\right)=L\left(\gamma_{3}, \gamma_{1}\right)=7 .
$$

$\Phi_{2} \mid b_{2}$ holds in certain subseries of the series $S_{1, p}^{\sharp}, E_{3, p}, Z_{1, p}, W_{1, p}$ and $S_{1, p}$. In these cases define

$$
\gamma_{2}:=v\left(\beta_{2},-1\right):=\frac{b_{2}}{\Phi_{2}}\left(M_{h}\right)\left(\beta_{2}\right)
$$

and calculate $L\left(\gamma_{2}, \gamma_{2}\right)$ using (2.17): $L\left(\gamma_{2}, \gamma_{2}\right)=\frac{b_{2}}{\Phi_{2}}(-1) \cdot L\left(\gamma_{2}, \beta_{2}\right)$. 


\begin{tabular}{lll} 
series & Condition for $\Phi_{2} \mid b_{2}$ & $L\left(\gamma_{2}, \gamma_{2}\right)$ \\
\hline$S_{1, p}^{\sharp}$ & $p \equiv 0(2)$ & $5+\frac{p}{2}$ \\
$E_{3, p}$ & $p \equiv 0(2)$ & $18+2 p$ \\
$Z_{1, p}$ & $p \equiv 0(2)$ & $14+2 p$ \\
$W_{1, p}$ & $p \equiv 1(2)$ & $12+2 p$ \\
$S_{1, p}$ & $p \equiv 0(2)$ & $10+2 p$
\end{tabular}

In table (5.20), the first line for $S_{1, p}^{\sharp}$ is the case $p \equiv 0(4)$, the second line is the case $p \equiv 2(4)$.

$$
\begin{array}{ll}
\text { series } & \gamma_{2} \\
\hline S_{1, p}^{\sharp} & -e_{2}+e_{4}+e_{5}+e_{6}-e_{7}+\sum_{j=1}^{2+p / 4}\left(e_{7+2 j}+e_{10+\frac{p}{2}+2 j}\right) \\
& -e_{4}+e_{5}+\sum_{j=1}^{(6+p) / 4}\left(-e_{8+2 j}+e_{11+\frac{p}{2}+2 j}\right) \\
E_{3, p} & -e_{2}+2 e_{5}+e_{6}-e_{7}+e_{9}+2 \sum_{j=1}^{4+p / 2} e_{8+2 j} \\
Z_{1, p} & -e_{2}+2 e_{5}+e_{6}-e_{7}+e_{10}+2 \sum_{j=1}^{3+p / 2} e_{9+2 j} \\
W_{1, p} & -2 e_{3}+e_{4}+e_{5}+e_{9}+e_{11}+e_{13}+e_{15}+2 \sum_{j=1}^{(1+p) / 2} e_{14+2 j} \\
S_{1, p} & 2\left(-e_{1}-e_{2}+e_{4}+e_{5}+e_{6}\right)-e_{7}-e_{8} \\
\quad & \quad+e_{9}+e_{11}+e_{12}+e_{14}-2 \sum_{j=1}^{p / 2} e_{14+2 j}
\end{array}
$$

In the subseries of $E_{3, p}, W_{1, p}$ and $S_{1, p}$ with $\Phi_{2} \mid b_{2}$, one sees

$$
\widetilde{\gamma}_{2}:=\frac{1}{2}\left(\gamma_{1}+\gamma_{2}\right) \stackrel{!}{\epsilon} M l(f) .
$$

In the subseries of $Z_{1, p}$ with $\Phi_{2} \mid b_{2}$, one sees

$$
\widetilde{\gamma}_{2}:=\frac{1}{2}\left(\gamma_{1}+\gamma_{2}-3 \gamma_{3}\right) \stackrel{!}{\epsilon} M l(f) .
$$

Together with $\left[M l(f): B_{1} \oplus B_{2}\right]=2$ for these subseries, this shows

$$
\begin{aligned}
& M l(f)_{\Phi_{2}}=\mathbb{Z} \gamma_{1} \oplus \mathbb{Z} \widetilde{\gamma}_{2} \quad \text { for } E_{3,2 q}, W_{1,2 q-1}, S_{1,2 q}, \\
& M l(f)_{\Phi_{2}}=\mathbb{Z}\left(\gamma_{1}-2 \gamma_{3}\right) \oplus \mathbb{Z} \widetilde{\gamma}_{2} \oplus \mathbb{Z} \gamma_{3} \quad \text { for } Z_{1,2 q} .
\end{aligned}
$$

For $S_{1,2 q}^{\sharp}, M l(f)=B_{1} \oplus B_{2}$ gives $M l(f)_{\Phi_{2}}=\mathbb{Z} \gamma_{1} \oplus \mathbb{Z} \gamma_{2}$. The matrices of $L$ for these bases of $M l(f)_{\Phi_{2}}$ in these cases are

$$
\begin{array}{cc}
S_{1,2 q}^{\sharp} & E_{3,2 q} \\
\left(\begin{array}{cc}
5 & 0 \\
0 & 5+q
\end{array}\right) & \left(\begin{array}{cc}
6 & 3 \\
3 & 6+q
\end{array}\right) \\
W_{1,2 q-1} & \left(\begin{array}{ccc}
5 & 2 & 1 \\
2 & 5+q & -1 \\
1 & -1 & 3
\end{array}\right) \\
\left(\begin{array}{cc}
6 & 3 \\
3 & 4+q
\end{array}\right) & \left(\begin{array}{cc}
10 & 5 \\
5 & 5+q
\end{array}\right)
\end{array}
$$


These matrices are positive definite. The corresponding quadratic forms $\left(x_{1} x_{2}\right)$ (matrix) $\left(\begin{array}{l}x_{1} \\ x_{2}\end{array}\right)$ respectively $\left(x_{1} x_{2} x_{3}\right)$ (matrix) $\left(\begin{array}{l}x_{1} \\ x_{2} \\ x_{3}\end{array}\right)$ are

$$
\begin{array}{rc}
5 x_{1}^{2}+(5+q) x_{2}^{2} & \text { for } S_{1,2 q}^{\sharp} \\
3 x_{1}^{2}+3\left(x_{1}+x_{2}\right)^{2}+(3+q) x_{2}^{2} & \text { for } E_{3,2 q} \\
\left(2 x_{1}+x_{2}\right)^{2}+\left(x_{1}+x_{3}\right)^{2} & \\
+\left(x_{2}-x_{3}\right)^{2}+(3+q) x_{2}^{2}+x_{3}^{2} & \text { for } Z_{1,2 q} \\
3 x_{1}^{2}+3\left(x_{1}+x_{2}\right)^{2}+(1+q) x_{2}^{2} & \text { for } W_{1,2 q-1} \\
5 x_{1}^{2}+5\left(x_{1}+x_{2}\right)^{2}+q x_{2}^{2} & \text { for } S_{1,2 q}
\end{array}
$$

This shows

$$
\left\{a \in M l(f)_{\Phi_{2}} \mid L(a, a)=L\left(\gamma_{1}, \gamma_{1}\right)\right\}=\left\{ \pm \gamma_{1}\right\} .
$$

for $W_{1,2 q-1}$ with $q \neq 2$, for $S_{1,2 q}$ with $q \neq 5$, and for all $S_{1,2 q}^{\sharp}$ and $E_{3,2 q}$. It shows for $Z_{1,2 q}$

$$
\begin{aligned}
& \left\{a \in M l(f)_{\Phi_{2}} \mid L(a, a)=3\right\}=\left\{ \pm \gamma_{3}\right\} \\
& \left\{a \in M l(f)_{\Phi_{2}} \mid L(a, a)=5\right\}=\left\{ \pm\left(\gamma_{1}-2 \gamma_{3}\right)\right\}
\end{aligned}
$$

All this implies

$$
\begin{aligned}
\operatorname{Aut}\left(M l(f)_{\Phi_{2}}, L\right)= & \left\{ \pm\left.\mathrm{id}\right|_{\mathbb{Z} \gamma_{1}}\right\} \times\left\{ \pm\left.\mathrm{id}\right|_{\mathbb{Z} \gamma_{2}}\right\} \quad \text { for } S_{1,2 q}^{\sharp}, \\
& \text { for } E_{3,2 q}, \text { for } S_{1,2 q} \text { with } q \neq 5, \\
& \text { and for } W_{1,2 q-1} \text { with } q \neq 2, \\
\operatorname{Aut}\left(M l(f)_{\Phi_{2}}, L\right)= & \left\{ \pm\left.\mathrm{id}\right|_{\mathbb{Z} \gamma_{1} \oplus \mathbb{Z} \gamma_{3}}\right\} \times\left\{ \pm\left.\mathrm{id}\right|_{\mathbb{Z} \gamma_{2}}\right\} \text { for } Z_{1,2 q} .
\end{aligned}
$$

In the cases $S_{1,2 q-1}^{\sharp}, E_{3,2 q-1}, Z_{1,2 q-1}, W_{1,2 q}$ and $S_{1,2 q-1}$ with $\Phi_{2} \backslash b_{2}$,

$$
M l(f)_{\Phi_{2}}=\left(\widetilde{B}_{1}\right)_{\Phi_{2}} \text { and } \operatorname{Aut}\left(M l(f)_{\Phi_{2}}, L\right)=\{ \pm \mathrm{id}\} .
$$

Define

$$
\gamma_{4}:= \begin{cases}\gamma_{1} & \text { for } E_{3, p}, W_{1, p}, S_{1, p} \\ \gamma_{1}-3 \gamma_{3} & \text { for } Z_{1, p} .\end{cases}
$$

Then for $E_{3, p}, W_{1, p}$ with $p \neq 3, S_{1, p}$ with $p \neq 10, Z_{1, p}$

$$
g\left(\gamma_{4}\right)= \pm \gamma_{4} \quad \text { for } g \in G_{\mathbb{Z}}
$$

and for $E_{3, p}, W_{1, p}$ (including $\left.p=3\right), S_{1, p}$ (including $\left.p=10\right), Z_{1, p}$

$$
\widetilde{B}_{1} \oplus B_{2}=\left\{a \in M l(f) \mid L\left(a, \gamma_{4}\right) \equiv 0(2)\right\} .
$$

Here $\subset$ 5.35 follows from $L\left(B_{2}, \gamma_{4}\right)=0$ and $L\left(\beta_{1}, \gamma_{4}\right) \equiv 0(2)$ and in the case of $Z_{1, p} L\left(\beta_{3}, \gamma_{4}\right)=4$. Now $=$ in (5.35) follows from 
$L\left(M l(f), \gamma_{4}\right)=\mathbb{Z}$ and $\left[M l(f): \widetilde{B}_{1} \oplus B_{2}\right]=2$. Together (5.34) and (5.35) show that any $g \in G_{\mathbb{Z}}$ respects $\widetilde{B}_{1} \oplus B_{2}$, so

$$
G_{\mathbb{Z}} \subset \operatorname{Aut}\left(\widetilde{B}_{1} \oplus B_{2}, L\right)
$$

for $E_{3, p}, W_{1, p}$ with $p \neq 3, S_{1, p}$ with $p \neq 10$ and $Z_{1, p}$. We claim that (5.34) and thus (5.36) hold also for $W_{1,3}$. That will be proved in the subsection 5.7 .

It rests to show $\operatorname{Aut}\left(\widetilde{B}_{1} \oplus B_{2}, L\right) \subset G_{\mathbb{Z}}$ for the series $E_{3, p}, Z_{1, p}, W_{1, p}$, $S_{1, p}$. We will extend the definition of $\widetilde{\gamma}_{2}$ in such a way to the cases with $\Phi_{2} \backslash b_{2}$ that $\left(\widetilde{B}_{1} \oplus B_{2}\right)+\mathbb{Z} \cdot \widetilde{\gamma}_{2}=M l(f)$. And we will show $g\left(\widetilde{\gamma}_{2}\right) \in M l(f)$ for any $g \in \operatorname{Aut}\left(\widetilde{B}_{1} \oplus B_{2}, L\right)$. This implies $\operatorname{Aut}\left(\widetilde{B}_{1} \oplus B_{2}, L\right) \subset G_{\mathbb{Z}}$. The proof of $g\left(\widetilde{\gamma}_{2}\right) \in M l(f)$ requires a better control of $\operatorname{Aut}\left(\widetilde{B}_{1} \oplus B_{2}, L\right)$.

Consider all eight series and define

$$
b_{4}:=\frac{\operatorname{gcd}\left(b_{1}, b_{2}\right)}{\operatorname{gcd}\left(b_{1}, b_{2}, \Phi_{m}\right)}=\operatorname{gcd}\left(\frac{b_{1}}{\Phi_{m}}, b_{2}\right) \in \mathbb{Z}[t]
$$

Then

$$
b_{4}= \begin{cases}1 & \text { for } W_{1, p}^{\sharp}, S_{1,2 q-1}^{\sharp}, U_{1, p}, E_{3,2 q-1}, Z_{1,2 q-1}, \\ & Q_{2, p} \text { with } p \not \equiv 0(4), W_{1,2 q}, S_{1,2 q-1}, \\ \Phi_{2} & \text { for } S_{1,2 q}^{\sharp}, E_{3,2 q}, Z_{1,2 q}, W_{1,2 q-1} \text { with } q \neq \equiv 2(3), S_{1,2 q}, \\ \Phi_{4} & \text { for } Q_{2,4 s}, \\ \Phi_{6} \Phi_{2} & \text { for } W_{1,6 s-3} .\end{cases}
$$

We claim that in all cases except $S_{1,10}$, any $g \in G_{\mathbb{Z}} \cup \operatorname{Aut}\left(\widetilde{B}_{1} \oplus B_{2}, L\right)$ maps $\left(\widetilde{B}_{1}\right)_{b_{4}}$ to $\left(\widetilde{B}_{1}\right)_{b_{4}}$ and $\left(B_{2}\right)_{b_{4}}$ to $\left(B_{2}\right)_{b_{4}}$. In the cases with $b_{4}=1$ this is an empty statement as then $\left(\widetilde{B}_{1}\right)_{b_{4}}=\{0\}=\left(B_{2}\right)_{b_{4}}$. In the cases $Q_{2, p}$ with $p \equiv 0(4)$ and $W_{1,6 s-3}$, this will be shown in the subsections 5.6 and 5.7. In all other cases $b_{4}=\Phi_{2}$ and $\left(B_{2}\right)_{b_{4}}=\mathbb{Z} \cdot \gamma_{2}$ and

$$
\left(\widetilde{B}_{1}\right)_{b_{4}}= \begin{cases}\mathbb{Z} \cdot\left(\gamma_{1}-2 \gamma_{3}\right) \oplus \mathbb{Z} \cdot \gamma_{3} & \text { for } Z_{1,2 q} \\ \mathbb{Z} \cdot \gamma_{1} & \text { else. }\end{cases}
$$

Because $\left.\left(\widetilde{B}_{1} \oplus B_{2}\right)_{\Phi_{2}} \subset M l(f)_{\Phi_{2}},(5.27)-5.29\right)$ hold also with $\left(\widetilde{B}_{1} \oplus\right.$ $\left.B_{2}\right)_{\Phi_{2}}$ instead of $M l(f)_{\Phi_{2}}$. They characterize $\left(\widetilde{B}_{1}\right)_{\Phi_{2}}$ within $M l(f)_{\Phi_{2}}$ and within $\left(\widetilde{B}_{1} \oplus B_{2}\right)_{\Phi_{2}}$. Thus any $g \in G_{\mathbb{Z}} \cup \operatorname{Aut}\left(\widetilde{B}_{1} \oplus B_{2}, L\right)$ maps $\left(\widetilde{B}_{1}\right)_{\Phi_{2}}$ to itself, and then it maps also the $L$-orthogonal sublattice $\left(B_{2}\right)_{\Phi_{2}}$ to itself. 
For all eight series except $S_{1,10}$, this implies the following. For any $g \in G_{\mathbb{Z}} \cup \operatorname{Aut}\left(\widetilde{B}_{1} \oplus B_{2}, L\right)$

$$
\left.\begin{array}{cc}
g: \widetilde{B}_{1} \rightarrow \widetilde{B}_{1} \text { and } B_{2} \rightarrow B_{2} & \text { if } m \not p, \\
g:\left(\widetilde{B}_{1}\right)_{b_{1} / \Phi_{m}} \rightarrow\left(\widetilde{B}_{1}\right)_{b_{1} / \Phi_{m}} \\
g:\left(B_{2}\right)_{b_{2} / \Phi_{m}} \rightarrow\left(B_{2}\right)_{b_{2} / \Phi_{m}}
\end{array}\right\} \quad \begin{array}{r}
\text { if } m \mid p \text { and the } \\
\text { type is not } S_{1,10} .
\end{array}
$$

Now we want to apply lemma 2.8 to these Orlik blocks. One checks easily that all hypotheses are satisfied. Therefore

$$
\begin{aligned}
& \operatorname{Aut}\left(\widetilde{B}_{1} \oplus B_{2}, L\right) \\
= & \left\{ \pm\left. M_{h}^{k}\right|_{\widetilde{B}_{1}} \mid k \in \mathbb{Z}\right\} \times\left\{ \pm\left. M_{h}^{k}\right|_{B_{2}} \mid k \in \mathbb{Z}\right\} \quad \text { if } m \not p,
\end{aligned}
$$

and if $m \mid p$ and the type is not $S_{1,10}$, then $\operatorname{Aut}\left(\widetilde{B}_{1} \oplus B_{2}, L\right)$ projects to a subgroup of

$$
\begin{aligned}
& \operatorname{Aut}\left(\left(\widetilde{B}_{1}\right)_{b_{1} / \Phi_{m}}, L\right) \times \operatorname{Aut}\left(\left(B_{2}\right)_{b_{2} / \Phi_{m}}, L\right) \\
= & \left\{ \pm\left. M_{h}^{k}\right|_{\left(\widetilde{B}_{1}\right)_{b_{1} / \Phi_{m}}} \mid k \in \mathbb{Z}\right\} \times\left\{ \pm\left. M_{h}^{k}\right|_{\left(B_{2}\right)_{b_{2} / \Phi_{m}}} \mid k \in \mathbb{Z}\right\} .
\end{aligned}
$$

The group $\operatorname{Aut}\left(\widetilde{B}_{1} \oplus B_{2}, L\right)$ for $m \not p$ is generated by $M_{h},-\mathrm{id},\left.M_{h}\right|_{\widetilde{B}_{1}} \times$ id $\left.\right|_{B_{2}}$ and $\left(-\left.\mathrm{id}\right|_{\widetilde{B}_{1}}\right) \times\left.\mathrm{id}\right|_{B_{2}}$, and analogously for the group in (5.43) if $m \mid p$.

Now we extend the definition of $\gamma_{2}$. For $E_{3,2 q-1}, Z_{1,2 q-1}$ and $S_{1,2 q-1}$ define it as follows:

$$
\begin{aligned}
\gamma_{2} & :=e_{2}-e_{6}+e_{7}+e_{9} \text { for } E_{3,2 q-1}, \\
\gamma_{2} & :=e_{2}-e_{6}+e_{7}+e_{10} \text { for } Z_{1,2 q-1}, \\
\gamma_{2} & :=2\left(-e_{1}-e_{2}+\sum_{j \in\{4,5,6\}} e_{j}\right)-e_{7}-e_{8}+\sum_{j \in\{9,11,12,14\}} e_{j} \text { for } S_{1,2 q-1} .
\end{aligned}
$$

(5.105), 5.110) and (5.162) show $\gamma_{2} \in B_{2}$. For $W_{1,2 q}$ (so $p=2 q$ ) define

$$
\begin{aligned}
\gamma_{2} & :=\left(t^{p}(t+1) \Phi_{12}+\sum_{j=0}^{p-1} t^{j}\right)\left(M_{h}\right)\left(e_{16}\right) \\
& =\left(t^{p}\left(1+t-t^{2}-t^{3}+t^{4}+t^{5}\right)+\sum_{j=0}^{p-1} t^{j}\right)\left(M_{h}\right)\left(e_{16}\right) \\
& =-2 e_{2}+2 e_{6}-2 e_{7}+e_{4}+e_{5}+e_{9}-e_{11}+e_{13}-e_{15} .
\end{aligned}
$$

Observe that in the case $12 \mid p, \Phi_{12}$ divides $\sum_{j=0}^{p-1} t^{j}$ so that then $\gamma_{2} \in$ $\Phi_{12}\left(M_{h}\right)\left(B_{2}\right)=\left(B_{2}\right)_{b_{2} / \Phi_{12}}$. In all four cases $\frac{1}{2}\left(\gamma_{4}+\gamma_{2}\right) \in M l(f)$. 
Now for the series $E_{3, p}, Z_{1, p}, W_{1, p}$ and $S_{1, p}$

$$
\begin{aligned}
& \gamma_{4} \in\left(B_{1}\right)_{\Phi_{2}}, \quad \begin{cases}\gamma_{2} \in B_{2} & \text { if } m \backslash p \\
\gamma_{2} \in\left(B_{2}\right)_{b_{2} / \Phi_{m}} & \text { if } m \mid p,\end{cases} \\
& \widetilde{\gamma}_{2}:=\frac{1}{2}\left(\gamma_{4}+\gamma_{2}\right) \stackrel{!}{\in} M l(f), \\
& M l(f)=\left(\widetilde{B}_{1} \oplus B_{2}\right)+\mathbb{Z} \widetilde{\gamma}_{2}, \\
& \left(\left.M_{h}\right|_{\widetilde{B}_{1}} \times\left.\mathrm{id}\right|_{B_{2}}\right)\left(\widetilde{\gamma}_{2}\right)=\left(\left(-\left.\mathrm{id}\right|_{\widetilde{B}_{1}}\right) \times\left.\mathrm{id}\right|_{B_{2}}\right)\left(\widetilde{\gamma}_{2}\right) \\
& =\frac{1}{2}\left(-\gamma_{4}+\gamma_{2}\right)=-\gamma_{4}+\widetilde{\gamma}_{2} \in M l(f) .
\end{aligned}
$$

Therefore any $g \in \operatorname{Aut}\left(\widetilde{B}_{1} \oplus B_{2}, L\right)$ maps $\widetilde{\gamma}_{2}$ to an element of $M l(f)$. Thus it maps $M l(f)$ to $M l(f)$, thus $g \in G_{\mathbb{Z}}$. This finishes the proof of (5.4) and of part (a) for all series except $Q_{2, p}$ and $W_{1,6 s-3}$ and $S_{1,10}$. For $Q_{2, p}$ and $W_{1,6 s-3}$ and $S_{1,10}$ see the subsections 5.6, 5.7 and 5.8.

(b) This follows immediately from (5.4) and (5.42). The subsections 5.6 and 5.7 establish (5.4) and (5.42) also for the series $Q_{2, p}$ and $W_{1,6 s-3}$.

(c) Now we consider the eight subseries with $m \mid p$. Write $p=m \cdot r$ with $r \in \mathbb{Z}_{\geq 1}$. Recall $\zeta=e^{2 \pi i / m}$, and recall that $\mathbb{Z}[\zeta]$ is a principal ideal domain (lemma 2.11). In the following, $\xi$ will be any primitive $m$-th unit root.

Formula 2.24 in lemma 2.12 (b) applies with $\Lambda=M l(f), \Lambda^{(1)}=$ $\widetilde{B}_{1} \oplus B_{2}, p=\Phi_{m}$, and gives

$$
M l(f)_{\Phi_{m}}=\left(\widetilde{B}_{1} \oplus B_{2}\right)_{\Phi_{m}}=\left(B_{1} \oplus B_{2}\right)_{\Phi_{m}}=\left(B_{1}\right)_{\Phi_{m}} \oplus\left(B_{2}\right)_{\Phi_{m}}
$$

Therefore the space

$$
M l(f)_{\xi, \mathbb{Z}[\zeta]}:=M l(f)_{\xi} \cap M l(f)_{\mathbb{Z}[\zeta]}
$$

is a free $\mathbb{Z}[\zeta]$-module of rank 2 with basis $v_{1, \xi}, v_{2, \xi}$ with

$$
v_{j, \xi}:=v\left(\beta_{j}, \xi\right)=\frac{b_{j}}{t-\xi}\left(M_{h}\right)\left(\beta_{j}\right) \quad \text { for } j=1,2
$$

(see 2.16 for the notion $v\left(\beta_{j}, \xi\right)$ ). Observe $v_{j, \bar{\xi}}=\overline{v_{j, \xi}}$.

The proof of part (c) will consist of four steps. Step 1 calculates the values of the hermitian form $h_{\xi}$ from lemma 2.2 on a suitable $\mathbb{Z}[\zeta]$-basis

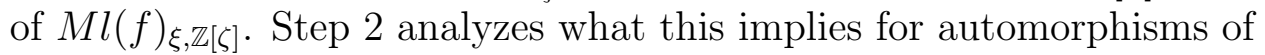
the pair $\left(M l(f)_{\xi, \mathbb{Z}[\zeta]}, L\right)$ and thus gives a first approximation to $\Psi\left(G_{\mathbb{Z}}\right)$. Step 3 uses (5.5) for $S_{1,10}$ and (5.41) for all other singularities and the Orlik block structure of the blocks $B_{j}$ to control the action of $g \in G_{\mathbb{Z}}$ on all eigenspaces simultaneously. It will prove (5.9). Step 4 combines the steps 2 and 3 with results from section 3 and shows that $\Psi\left(G_{\mathbb{Z}}\right)$ is an infinite Fuchsian group. 
Step 1: The form

$$
h_{\xi}: M l(f)_{\xi} \times M l(f)_{\xi} \rightarrow \mathbb{C}, \quad(a, b) \mapsto \sqrt{-\xi} \cdot L(a, \bar{b})
$$

from lemma 2.2 is hermitian. In this step it will be calculated with

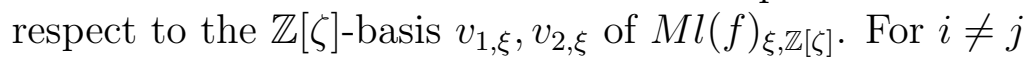

$$
h_{\xi}\left(v_{i, \xi}, v_{j, \xi}\right)=\sqrt{-\xi} \cdot L\left(v_{i, \xi}, v_{j, \bar{\xi}}\right)=0
$$

because of (5.3). $L\left(v_{j, \xi}, v_{j, \bar{\xi}}\right)$ will be calculated with (2.17),

$$
L\left(v_{j, \xi}, v_{j, \bar{\xi}}\right)=\frac{b_{j}}{t-\bar{\xi}}(\bar{\xi}) \cdot L\left(\frac{b_{j}}{t-\xi}\left(M_{h}\right)\left(\beta_{j}\right), \beta_{j}\right),
$$

first for $j=2$, then for $j=1$.

One calculates for all eight subseries:

\begin{tabular}{c|c|c|c|c|c|c|}
$k$ & 0 & 1 & 2 & $\cdots$ & $\operatorname{deg} b_{2}-1$ & $\operatorname{deg} b_{2}$ \\
\hline$L\left(M_{h}^{k}\left(\beta_{2}\right), \beta_{2}\right)$ & 1 & -1 & 0 & $\cdots$ & 0 & 0 if $r_{I}=1,-1$ if $r_{I} \geq 2$
\end{tabular}

For the three subseries with $r_{I}=1$ ( so $W_{1,12 r}^{\sharp}, S_{1,10 r}^{\sharp}, U_{1,9 r}$ )

$$
\begin{gathered}
\frac{b_{2}}{t-\xi}=\frac{t^{m+p}-1}{(t-\xi) \cdot \Phi_{1}}=\Phi_{1}^{-1} \cdot \sum_{j=0}^{m+p-1} \xi^{m+p-1-j} \cdot t^{j} \\
\frac{b_{2}}{t-\bar{\xi}}(\bar{\xi})=(\bar{\xi}-1)^{-1} \cdot(m+p) \cdot \xi=m(1+r)(\bar{\xi}-1)^{-1} \cdot \xi \\
L\left(\frac{b_{2}}{t-\xi}\left(M_{h}\right)\left(\beta_{2}\right), \beta_{2}\right)=(\xi-1)^{-1} \cdot \bar{\xi} \cdot(1-\bar{\xi})=\bar{\xi}^{2} \\
h_{\xi}\left(v_{2, \xi}, v_{2, \xi}\right)=m(1+r) \cdot(1-\xi)^{-1} \cdot \sqrt{-\xi}>0
\end{gathered}
$$

For the five subseries with $r_{I}=2$

$$
\begin{gathered}
\frac{b_{2}}{t-\xi}=\frac{t^{m / 2+p}+1}{t-\xi}=\sum_{j=0}^{m / 2+p-1} \xi^{m / 2+p-1-j} \cdot t^{j}, \\
\frac{b_{2}}{t-\bar{\xi}}(\bar{\xi})=\left(\frac{m}{2}+p\right)(-\xi)=\frac{m}{2}(1+2 r)(-\xi), \\
L\left(\frac{b_{2}}{t-\xi}\left(M_{h}\right)\left(\beta_{2}\right), \beta_{2}\right)=-\bar{\xi}(1-\bar{\xi}), \\
h_{\xi}\left(v_{2, \xi}, v_{2, \xi}\right)=\frac{m}{2}(1+2 r) \cdot(1-\bar{\xi}) \cdot \sqrt{-\xi}>0
\end{gathered}
$$


Now we turn to $h_{\xi}\left(v_{1, \xi}, v_{1, \xi}\right)$. One calculates for all eight series

\begin{tabular}{c|c|c|c|c|c|c|c|c|c|c|c|c|}
$k$ & 0 & 1 & 2 & 3 & 4 & 5 & 6 & 7 & 8 & 9 & 10 & 11 \\
$L\left(M_{h}^{k}\left(\beta_{1}\right), \beta_{1}\right)$ & & & & & & & & & & & & \\
\hline for $W_{1, p}^{\sharp}$ & 1 & -1 & 1 & 0 & 0 & 1 & & & & & & \\
for $S_{1, p}^{\sharp}$ & 1 & -1 & 0 & 1 & 0 & & & & & & & \\
for $U_{1, p}$ & 1 & -1 & 0 & 0 & 1 & 0 & -1 & 0 & 0 & & & \\
for $E_{3, p}$ & 1 & -1 & 1 & 0 & 1 & 0 & 1 & 0 & 1 & & & \\
for $Z_{1, p}$ & 1 & -1 & 0 & 0 & 1 & 0 & 0 & & & & & \\
for $Q_{2, p}$ & 1 & -1 & 0 & 1 & 0 & 0 & 0 & 0 & 0 & 0 & -1 & 0 \\
for $W_{1, p}$ & 3 & -3 & 2 & -1 & 0 & 1 & -1 & 1 & -1 & 0 & 1 & -2 \\
for $S_{1, p}$ & 2 & -2 & 0 & 1 & 0 & -1 & 1 & 0 & -1 & 0 & &
\end{tabular}

and

for $W_{1, p}^{\sharp} \quad \frac{b_{1}}{t-\xi}=\frac{\Phi_{12}}{t-\xi}=t^{3}+\xi t^{2}+\left(\xi^{2}-1\right) t+\left(\xi^{3}-\xi\right)$, for $S_{1, p}^{\sharp} \quad \frac{b_{1}}{t-\xi}=\frac{\Phi_{10} \Phi_{2}}{t-\xi}=\frac{t^{5}+1}{t-\xi}=t^{4}+\xi t^{3}+\xi^{2} t^{2}+\xi^{3} t+\xi^{4}$,

for $U_{1, p} \quad \frac{b_{1}}{t-\xi}=\frac{\Phi_{9}}{t-\xi}=\frac{t^{6}+t^{3}+1}{t-\xi}$

$$
=t^{5}+\xi t^{4}+\xi^{2} t^{3}+\left(\xi^{3}+1\right) t^{2}+\left(\xi^{4}+\xi\right) t+\left(\xi^{5}+\xi^{2}\right),
$$

for $E_{3, p} \quad \frac{b_{1}}{t-\xi}=\frac{\Phi_{18} \Phi_{2}}{t-\xi}=\frac{t^{7}+t^{6}-t^{4}-t^{3}+t+1}{t-\xi}=t^{6}+(\xi+1) t^{5}$

$$
+\left(\xi^{2}+\xi\right) t^{4}+\left(\xi^{6}+\xi^{2}\right) t^{3}+\left(\xi^{7}+\xi^{6}\right) t^{2}+\left(\xi^{8}+\xi^{7}\right) t+\xi^{8},
$$

for $Z_{1, p} \quad \frac{b_{1}}{t-\xi}=\frac{t^{7}+1}{t-\xi}=t^{6}+\xi t^{5}+\xi^{2} t^{4}+\xi^{3} t^{3}+\xi^{4} t^{2}+\xi^{5} t+\xi^{6}$,

for $Q_{2, p} \quad \frac{b_{1}}{t-\xi}=\frac{\Phi_{12} \Phi_{4} \Phi_{3}}{t-\xi}=\frac{t^{8}+t^{7}+t^{6}+t^{2}+t+1}{t-\xi}$

$$
\begin{aligned}
& =t^{7}+(\xi+1) t^{6}+\left(\xi^{2}+\xi+1\right) t^{5}+\left(\xi^{3}+\xi^{2}+\xi\right) t^{4} \\
& +\left(\xi^{4}+\xi^{3}+\xi^{2}\right) t^{3}+\left(\xi^{5}+\xi^{4}+\xi^{3}\right) t^{2}+\left(\xi^{5}+\xi^{4}\right) t+\xi^{5},
\end{aligned}
$$




$$
\begin{aligned}
\text { for } W_{1, p} \quad & \frac{b_{1}}{t-\xi}=\frac{\Phi_{12} \Phi_{6} \Phi_{3} \Phi_{2}}{t-\xi}=\frac{t^{9}+t^{8}+t^{5}+t^{4}+t+1}{t-\xi} \\
& =t^{8}+(\xi+1) t^{7}+\left(\xi^{2}+\xi\right) t^{6}+\left(\xi^{3}+\xi^{2}\right) t^{5}+\left(\xi^{3}+\xi^{2}\right) t^{4} \\
& +\left(\xi^{3}+\xi^{2}\right) t^{3}+\left(\xi^{4}+\xi^{3}\right) t^{2}+\left(\xi^{5}+\xi^{4}\right) t+\xi^{5}, \\
\text { for } S_{1, p} \quad & \frac{b_{1}}{t-\xi}=\frac{\Phi_{10} \Phi_{5} \Phi_{2}}{t-\xi}=\frac{\sum_{j=0}^{9} t^{j}}{t-\xi} \\
& =t^{8}+(\xi+1) t^{7}+\left(\xi^{2}+\xi+1\right) t^{6}+\left(\xi^{3}+\xi^{2}+\xi+1\right) t^{5} \\
& +\left(\xi^{4}+\xi^{3}+\xi^{2}+\xi+1\right) t^{4}+\left(\xi^{4}+\xi^{3}+\xi^{2}+\xi\right) t^{3} \\
& +\left(\xi^{4}+\xi^{3}+\xi^{2}\right) t^{2}+\left(\xi^{4}+\xi^{3}\right) t+\xi^{4} .
\end{aligned}
$$

This table and this list give the following values.

\begin{tabular}{c|c|c} 
& $\frac{b_{1}}{t-\bar{\xi}}(\bar{\xi})$ & $L\left(\frac{b_{1}}{t-\xi}\left(M_{h}\right)\left(\beta_{1}\right), \beta_{1}\right)$ \\
\hline$W_{1, p}^{\sharp}$ & $4 \bar{\xi}^{3}-2 \bar{\xi}=-2(\xi+\bar{\xi}) \xi^{2}$ & $\xi^{3}(1-\xi)$ \\
$S_{1, p}^{\sharp}$ & $5 \bar{\xi}^{4}=-5 \xi$ & $-\xi\left(\xi^{2}+\bar{\xi}^{2}-1\right)$ \\
$U_{1, p}$ & $6 \bar{\xi}^{5}+3 \bar{\xi}^{2}=3 \xi\left(\xi^{3}-1\right)$ & $-\xi^{6}\left(\xi^{2}+\bar{\xi}^{2}\right)$ \\
$E_{3, p}$ & $3\left(\bar{\xi}^{6}+\bar{\xi}^{5}+\bar{\xi}^{9}+\bar{\xi}^{8}\right)=-3(\xi+1)\left(\xi^{3}+1\right)$ & $\xi^{2}(\xi+\bar{\xi})\left(\xi^{2}+\bar{\xi}^{2}\right)$ \\
$Z_{1, p}$ & $7 \bar{\xi}^{6}=-7 \xi$ & $\xi^{2}\left(\xi^{4}+\bar{\xi}^{4}+1\right)$ \\
$Q_{2, p}$ & $6\left(\bar{\xi}^{7}+\bar{\xi}^{6}+\bar{\xi}^{5}\right)=-6(\xi+\bar{\xi}+1)$ & $\xi^{2}(\xi+1)=(1-\xi)^{-1}$ \\
$W_{1, p}$ & $4\left(\bar{\xi}^{8}+\bar{\xi}^{7}+\bar{\xi}^{6}+\bar{\xi}^{5}\right)=4 \bar{\xi}^{7}(1+\xi)(\xi+\bar{\xi})$ & $\xi^{3}(\xi-1)(\bar{\xi}-1)$ \\
$S_{1, p}$ & $5\left(\bar{\xi}^{8}+\bar{\xi}^{7}+\bar{\xi}^{6}+\bar{\xi}^{5}+\bar{\xi}^{4}\right)$ & $-1+\xi+\xi^{2}-2 \xi^{3}+\xi^{4}$
\end{tabular}

With $h_{\xi}\left(v_{1, \xi}, v_{1, \xi}\right)=\sqrt{-\xi} \cdot L\left(v_{1, \xi}, v_{1, \bar{\xi}}\right)$ and (5.54) and the information on the rings $\mathbb{Z}[\zeta]$ in lemma 2.11 , we obtain the following values.

\begin{tabular}{c|r} 
& $h_{\xi}\left(v_{1, \xi}, v_{1, \xi}\right)$ \\
\hline$W_{1, p}^{\sharp}$ & $(-2)(\xi+\bar{\xi}) \cdot(1-\bar{\xi}) \sqrt{-\xi}$ \\
$S_{1, p}^{\sharp}$ & $5\left(\xi^{2}+\bar{\xi}^{2}\right)\left(\xi^{2}+\bar{\xi}^{2}-1\right) \cdot(1-\xi)^{-1} \sqrt{-\xi}$ \\
$U_{1, p}$ & $3\left(\xi^{4}+\bar{\xi}^{4}+1\right) \cdot(1-\bar{\xi}) \sqrt{-\xi}$ \\
$E_{3, p}$ & $(-3)(1+\xi)(1+\bar{\xi})(\xi+\bar{\xi}-1) \cdot(1-\xi)^{-1} \sqrt{-\xi}$ \\
$Z_{1, p}$ & $(-7)\left(\xi^{2}+\bar{\xi}^{2}\right) \cdot(1-\bar{\xi}) \sqrt{-\xi}$ \\
$Q_{2, p}$ & $(-6)(\xi+\bar{\xi}+1) \cdot(1-\xi)^{-1} \sqrt{-\xi}$ \\
$W_{1, p}$ & $(-4)(\xi+\bar{\xi}) \cdot(1-\bar{\xi}) \sqrt{-\xi}$ \\
$S_{1, p}$ & $(-10)\left(\xi^{2}+\bar{\xi}^{2}\right) \cdot(1-\bar{\xi}) \sqrt{-\xi}$
\end{tabular}

Here observe that as in $(5.58)$ and $(5.62)(1-\bar{\xi}) \sqrt{-\xi}>0$ and $(1-$ $\xi)^{-1} \sqrt{-\xi}>0$. In each of the eight cases we find

$$
\begin{array}{ll}
h_{\xi}\left(v_{1, \xi}, v_{1, \xi}\right)>0 & \text { for } \xi \notin\{\zeta, \bar{\zeta}\}, \\
h_{\xi}\left(v_{1, \xi}, v_{1, \xi}\right)<0 & \text { for } \xi \in\{\zeta, \bar{\zeta}\},
\end{array}
$$


and

$$
L\left(v_{1, \xi}, \beta_{1}\right)=L\left(\frac{b_{1}}{t-\xi}\left(M_{h}\right)\left(\beta_{1}\right), \beta_{1}\right) \in \mathbb{Z}[\zeta]^{*} .
$$

Step 2: Define for each of the eight series

$$
b_{5}:=\frac{b_{1}}{\Phi_{m}} \in \mathbb{Z}[t] \quad \text { unitary. }
$$

Then

\begin{tabular}{c|c|c|c|c|c|c|c|c} 
series & $W_{1, p}^{\sharp}$ & $S_{1, p}^{\sharp}$ & $U_{1, p}$ & $E_{3, p}$ & $Z_{1, p}$ & $Q_{2, p}$ & $W_{1, p}$ & $S_{1, p}$ \\
\hline$b_{5}$ & 1 & $\Phi_{2}$ & 1 & $\Phi_{2}$ & $\Phi_{2}$ & $\Phi_{4} \Phi_{3}$ & $\Phi_{6} \Phi_{3} \Phi_{2}$ & $\Phi_{5} \Phi_{2}$
\end{tabular}

and

$$
b_{5}(\xi) / b_{5}(\bar{\xi}) \in\left\{ \pm \xi^{k} \mid k \in \mathbb{Z}\right\} .
$$

Define for each of the eight subseries with $m \mid p$

$$
b_{6}:=\frac{b_{2}}{\Phi_{m}} \in \mathbb{Z}[t] \quad \text { unitary }
$$

and

$$
\begin{aligned}
w(\xi) & :=-\frac{h_{\xi}\left(v_{2, \xi}, v_{2, \xi}\right)}{h_{\xi}\left(v_{1, \xi}, v_{1, \xi}\right)}=-\frac{\frac{b_{2}}{t-\bar{\xi}}(\bar{\xi}) \cdot L\left(v_{2, \xi}, \beta_{2}\right)}{\frac{b_{1}}{t-\bar{\xi}}(\bar{\xi}) \cdot L\left(v_{1, \xi}, \beta_{1}\right)} \\
& =-\frac{b_{6}}{b_{5}}(\bar{\xi}) \cdot \frac{L\left(v_{2, \xi}, \beta_{2}\right)}{L\left(v_{1, \xi}, \beta_{1}\right)}
\end{aligned}
$$

Then

$$
b_{5}(\bar{\xi}) w(\xi)=b_{6}(\bar{\xi}) \cdot \frac{L\left(v_{2, \xi}, \beta_{2}\right)}{L\left(v_{1, \xi}, \beta_{1}\right)} \in \mathbb{Z}[\zeta]
$$

It is in $\mathbb{Z}[\zeta]$ because of 5.66 . The following table lists $w(\xi)$.

$$
\begin{array}{c|l} 
& w(\xi) \\
\hline W_{1, p}^{\sharp} & (1+r)(+6)[(1-\xi)(1-\bar{\xi})(\xi+\bar{\xi})]^{-1} \\
S_{1, p}^{\sharp} & (1+r)(-2)\left[\left(\xi^{2}+\bar{\xi}^{2}\right)\left(\xi^{2}+\bar{\xi}^{2}-1\right)\right]^{-1} \\
U_{1, p} & (1+r)(-3)\left[(1-\xi)(1-\bar{\xi})\left(\xi^{4}+\bar{\xi}^{4}+1\right)\right]^{-1} \\
E_{3, p} & (1+2 r)(+3)(1-\xi)(1-\bar{\xi})[(1+\xi)(1+\bar{\xi})(\xi+\bar{\xi}-1)]^{-1}(5.72) \\
Z_{1, p} & (1+2 r)(+1)\left[\xi^{2}+\bar{\xi}^{2}\right]^{-1} \\
Q_{2, p} & (1+2 r)(+1)(1-\bar{\xi})(1-\bar{\xi})[\xi+\bar{\xi}+1]^{-1} \\
W_{1, p} & (1+2 r)\left(+\frac{3}{2}\right)[\xi+\bar{\xi}]^{-1} \\
S_{1, p} & (1+2 r)\left(+\frac{1}{2}\right)\left[\xi^{2}+\bar{\xi}^{2}\right]^{-1}
\end{array}
$$


The inequalities (5.58) (5.62) (5.64) (5.65) give

$$
w(\xi)\left\{\begin{aligned}
<0 & \text { for } \xi \notin\{\zeta, \bar{\zeta}\} \\
>0 & \text { for } \xi \in\{\zeta, \bar{\zeta}\}
\end{aligned}\right.
$$

Using the $\mathbb{Z}[\zeta]$-basis $v_{1, \xi}, v_{2, \xi}$ of $M l(f)_{\xi, \mathbb{Z}[\zeta]}$, the automorphism group $\operatorname{Aut}\left(M l\left((f)_{\xi, \mathbb{Z}[\zeta]}, h_{\xi}\right)\right.$ can be identified with the matrix group

$$
\begin{aligned}
\{A(\xi) \in G L(2, \mathbb{Z}[\zeta]) & \mid \\
\left(\begin{array}{cc}
-1 & 0 \\
0 & w(\xi)
\end{array}\right) & \left.=A(\xi)^{t} \cdot\left(\begin{array}{cc}
-1 & 0 \\
0 & w(\xi)
\end{array}\right) \cdot \overline{A(\xi)}\right\} .
\end{aligned}
$$

The isomorphism is $A(\xi) \mapsto g$ with

$$
g\left(v_{1, \xi}, v_{2, \xi}\right)=\left(v_{1, \xi}, v_{2, \xi}\right) \cdot A(\xi) .
$$

The inequalities (5.73) and theorem 3.2 tell that the matrix group in the case of $\xi=\zeta$ projects to an infinite Fuchsian group. Additionally, 3.2 tells that the elements of the matrix group for any $\xi$ can be represented by triples $(a(\xi), c(\xi), \delta(\xi)) \in \mathbb{Z}[\zeta]^{2} \times\left\{ \pm \zeta^{k} \mid k \in \mathbb{Z}\right\}$ with

$$
a(\xi) a(\bar{\xi})-1=w(\xi) \cdot c(\xi) c(\bar{\xi}),
$$

where

$$
A(\xi)=\left(\begin{array}{cc}
a(\xi) & w(\xi) \cdot c(\bar{\xi}) \cdot \delta(\xi) \\
c(\xi) & a(\bar{\xi}) \cdot \delta(\xi)
\end{array}\right)
$$

This gives a first approximation of $\Psi\left(G_{\mathbb{Z}}\right)$. It took into account only the eigenspace $M l(f)_{\xi, \mathbb{Z}[\zeta]}$ and the pairing $h_{\xi}$ which $L$ and complex conjugation induce on it.

Step 3: Now (5.9) will be shown. We will use that the $B_{j}$ are Orlik blocks and lemma 2.8 and $(5.5)$ for $S_{1,10}$ and (5.43) for all other singularities.

Let $g \in \operatorname{ker} \Psi \subset G_{\mathbb{Z}}$, i.e. $\left.g\right|_{M l(f)_{\zeta}} \in \mathbb{C}^{*} \cdot$ id. Then $\left.g\right|_{M l(f)_{\xi}} \in \mathbb{C}^{*} \cdot$ id for all $\xi$ with $\Phi_{m}(\xi)=0$, and

$$
g\left(\left(B_{j}\right)_{\Phi_{m}}\right)=\left(B_{j}\right)_{\Phi_{m}} \quad \text { for } j=1,2 .
$$

Now $g\left(B_{j}\right)=B_{j}$ for $j=1,2$ follows in the case $S_{1,10}$ from $(5.5)$. For all other singularities $g\left(B_{j}\right)=B_{j}$ for $j=1,2$ follows with (5.43) (and (5.32) for $B_{3}$ in the case $Z_{1,14 r}$ ).

We want to apply lemma 2.8 to the Orlik blocks $B_{1}$ and $B_{2}$. One checks easily that all hypotheses are satisfied. In the case $Z_{1,14 r} B_{3}$ is glued to $B_{1}$ by (5.32). Therefore in all cases

$$
g=\left.\left(\varepsilon_{1} \cdot M_{h}^{k_{1}}\right)\right|_{B_{1}} \times\left.\left(\varepsilon_{2} \cdot M_{h}^{k_{2}}\right)\right|_{B_{2}}
$$


for some $\varepsilon_{1}, \varepsilon_{2} \in\{ \pm 1\}$ and $k_{1}, k_{2} \in \mathbb{Z}$. Now consider

$$
\tilde{g}:=\varepsilon_{2} \cdot M_{h}^{-k_{2}} \circ g
$$

It satisfies

$$
\begin{array}{r}
\left.\widetilde{g}\right|_{B_{1}}=\left.\varepsilon_{1} \varepsilon_{2} \cdot M_{h}^{k_{1}-k_{2}}\right|_{B_{1}},\left.\quad \widetilde{g}\right|_{B_{2}}=\mathrm{id},\left.\quad \widetilde{g}\right|_{M l(f)_{\xi}} \in \mathbb{C}^{*} \cdot \mathrm{id}, \\
\text { thus }\left.\tilde{g}\right|_{M l(f)_{\xi}}=\mathrm{id},\left.\quad \widetilde{g}\right|_{M l(f)_{\Phi_{m}}}=\mathrm{id} .
\end{array}
$$

Comparison with table (5.1) shows

$$
\begin{aligned}
& \widetilde{g}=\text { id for the first } 5 \text { series in }(5.1) \\
& \widetilde{g}=\text { id or } \widetilde{g}=-M_{h}^{\frac{m}{2}(1+2 r)} \quad \text { for the last } 3 \text { series in } 5.1 \text {. }
\end{aligned}
$$

In any case, $\widetilde{g}$ and $g$ are in $\left\{ \pm M_{h}^{k} \mid k \in \mathbb{Z}\right\}$, and thus ker $\Psi=\left\{ \pm M_{h}^{k} \mid k \in\right.$ $\mathbb{Z}\}$.

Step 4: By step 2, $\Psi\left(G_{\mathbb{Z}}\right)$ is a subgroup of an infinite Fuchsian group and therefore itself a Fuchsian group. It rests to show that it is an infinite group. By step 3, the kernel of $\Psi: G_{\mathbb{Z}} \rightarrow \Psi\left(G_{\mathbb{Z}}\right)$ is $\left\{ \pm M_{h}^{k} \mid k \in \mathbb{Z}\right\}$, so it is finite. Therefore it rests to show that $G_{\mathbb{Z}}$ is infinite. We will see that the subgroup of elements $g \in G_{\mathbb{Z}}$ with

$$
\begin{aligned}
g & =\text { id on any eigenspace } M l(f)_{\lambda} \text { with } \Phi_{m}(\lambda) \neq 0, \\
\text { i.e. } g & =\text { id on }\left(\widetilde{B}_{1}\right)_{b_{5}} \text { and on }\left(B_{2}\right)_{b_{6}} .
\end{aligned}
$$

is infinite.

Consider an element $g \in G_{\mathbb{Z}}$ with (5.82). For all singularities except $S_{1,10}$ (5.4) holds. For $S_{1,10}$ (5.82) implies $g\left(\gamma_{4}\right)= \pm \gamma_{4}$, and then (5.36) gives $g \in \operatorname{Aut}\left(B_{1} \oplus B_{2}, L\right)$. In the case of the series $Z_{1,14 r}$, the element $g$ maps $B_{1} \oplus B_{2}$ to itself because $\left(B_{1} \oplus B_{2}\right)_{\mathbb{C}}$ contains $\operatorname{ker} \Phi_{m}\left(M_{h}\right)$. In any case, lemma 2.7 applies with $k=2, \Lambda^{(1)}=B_{1}, \Lambda^{(2)}=B_{2}, e^{(1)}=$ $\beta_{1}, e^{(2)}=\beta_{2}, p_{0}=\Phi_{m}$. By 2.20 there are unique polynomials $p_{i j} \in$ $\mathbb{Z}[t]_{<\operatorname{deg} b_{i}}$ for $i=1,2$ with

$$
g\left(\beta_{j}\right)=p_{1 j}\left(M_{h}\right)\left(\beta_{1}\right)+p_{2 j}\left(M_{h}\right)\left(\beta_{2}\right)
$$

and

$$
\begin{array}{ll}
p_{11}=1+b_{5} \cdot q_{11}, & p_{12}=b_{5} \cdot q_{12}, \\
p_{21}=b_{6} \cdot q_{21}, & p_{22}=1+b_{6} \cdot q_{22}
\end{array}
$$

for suitable polynomials $q_{i j} \in \mathbb{Z}[t]_{<\varphi(m)}$.

$g$ restricts to an automorphism of the pair $\left.\left(B_{1} \oplus B_{2}\right)_{\Phi_{m}}, L\right)$. By (2.21), the matrix $A(\xi)$ from 5.75 in step 2 takes the form

$$
A(\xi)=\left(\begin{array}{cc}
1+b_{5}(\xi) q_{11}(\xi) & b_{6}(\xi) q_{12}(\xi) \\
b_{5}(\xi) q_{21}(\xi) & 1+b_{6}(\xi) q_{22}(\xi)
\end{array}\right) .
$$

By step 2, this matrix $A(\xi)$ satisfies (5.76) and (5.77). 
Vice versa, any polynomials $q_{i j} \in \mathbb{Z}[t]_{<\varphi(m)}$ for $i=1,2$ such that the matrix in (5.85) satisfies (5.76) and (5.77), give rise via (5.84) and (5.83) to an element $g \in G_{\mathbb{Z}}$ with (5.82).

We have to prove existence of infinitely many polynomials $q_{i j} \in$ $\mathbb{Z}[t]_{<\varphi(m)}$ such that the matrix in (5.85) satisfies $(5.76)$ and $(5.77)$ and that $q_{12}(\xi) \neq 0$ and $q_{21}(\xi) \neq 0$. We start by defining

$$
w_{0}(\xi):=w(\xi) b_{5}(\xi) b_{5}(\bar{\xi}) \in \mathbb{Z}[\zeta] \cap \mathbb{R}
$$

and asking for infinitely many solutions $a(\xi), f(\xi) \in \mathbb{Z}[\zeta] \cap \mathbb{R}$ of the Pell equation

$$
a(\xi)^{2}-1=w_{0}(\xi) \cdot f(\xi)^{2}
$$

with the additional condition

$$
w_{0}(\xi) \quad \mid a(\xi)-1
$$

Such solutions exist due to lemma 3.3. They give rise to the elements

$$
\begin{aligned}
q_{11}(\xi) & :=\frac{a(\xi)-1}{b_{5}(\xi)}, \quad q_{12}(\xi):=f(\xi) \cdot \frac{w(\xi) b_{5}(\bar{\xi})}{b_{6}(\xi)} \\
q_{21}(\xi) & :=f(\xi), \quad q_{22}(\xi):=\frac{a(\xi)-1}{b_{6}(\xi)}
\end{aligned}
$$

Here observe

$$
b_{6}(\xi)\left|w(\xi) b_{5}(\bar{\xi})\right| w_{0}(\xi) \mid a(\xi)-1
$$

see (5.71), (5.68) and (5.66). These elements come from unique polynomials $q_{i j} \in \mathbb{Z}[t]_{<\varphi(m)}$. These polynomials satisfy all desired properties.

5.1. The series $W_{1, p}^{\sharp}$. Here we only describe the case when $p=2 q$ is even. But one can easily obtain the odd case $p=2 q-1$ from that via replacing each $e_{\alpha+q}$ by $e_{\alpha-1+q}$ in the following lists. The monodromy acts on the distinguished basis $e_{1}, \ldots, e_{\mu}$ with the CDD in figure 1 as 


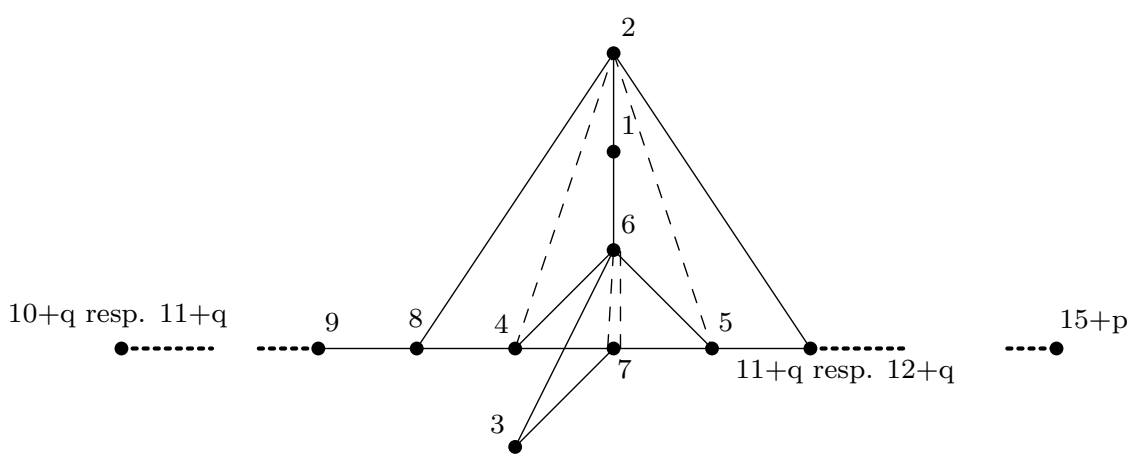

Figure 1. The CDD of a distinguished basis $e_{1}, \ldots, e_{\mu}$ for $W_{1,2 q-1}^{\sharp}$ resp. $W_{1,2 q}^{\sharp}$ from [Eb81, Tabelle 6 \& Abb. 16]

follows:

$$
\begin{aligned}
e_{1} & \mapsto-e_{1}-e_{2}+e_{3}+e_{4}+e_{5}+e_{6}, \\
e_{2} & \mapsto e_{1}+e_{2}+e_{8}+e_{12+q}, \\
e_{3} & \mapsto-e_{1}-e_{3}-e_{6}+e_{7}, \\
e_{4} & \mapsto e_{2}-e_{6}+e_{7}+e_{8}, \\
e_{5} & \mapsto e_{2}-e_{6}+e_{7}+e_{12+q}, \\
e_{6} & \mapsto e_{1}-2 e_{2}+e_{3}+e_{4}+e_{5}+3 e_{6}-2 e_{7}, \\
e_{7} & \mapsto-2 e_{2}+e_{3}+e_{4}+e_{5}+2 e_{6}-e_{7}, \\
e_{7+i} & \mapsto e_{8+i} \quad \text { for } 1 \leq i \leq 3+q, \\
e_{11+q} & \mapsto-e_{4}-e_{8}-e_{9}-\ldots-e_{11+q}, \\
e_{11+q+i} & \mapsto e_{12+q+i} \quad \text { for } 1 \leq i \leq 3+q, \\
e_{15+p} & \mapsto-e_{5}-e_{12+q}-\ldots-e_{15+p} .
\end{aligned}
$$


By table $(5.13)$ the generators of the Orlik blocks $B_{1}$ and $B_{2}$ are $\beta_{1}:=e_{3}$ and $\beta_{2}:=e_{8}$. The monodromy acts on them as follows:

$$
\begin{aligned}
e_{3} & \mapsto-e_{1}-e_{3}-e_{6}+e_{7} \mapsto e_{1}+e_{2}-e_{4}-e_{5}-e_{6} \\
& \mapsto-e_{1} \mapsto e_{1}+e_{2}-e_{3}-e_{4}-e_{5}-e_{6}, \\
e_{8} & \mapsto e_{9} \mapsto \ldots \mapsto e_{11+q} \mapsto-e_{4}-e_{8}-e_{9}-\ldots-e_{11+q} \\
& \mapsto-e_{2}+e_{4}+e_{6}-e_{7} \mapsto-e_{12+q} \mapsto-e_{13+q} \mapsto \ldots \mapsto-e_{15+p} \\
& \mapsto e_{5}+e_{12+q}+\ldots+e_{15+p} \mapsto e_{2}-e_{5}-e_{6}+e_{7} \mapsto e_{8} .
\end{aligned}
$$

Thus the characteristic polynomial of $M_{h}$ on $B_{j}$ is $b_{j}$, and the blocks are

$$
\begin{aligned}
& B_{1}=\left\langle e_{3}, e_{1}, e_{6}-e_{7}, e_{2}-e_{4}-e_{5}-e_{6}\right\rangle, \\
& B_{2}=\left\langle e_{8}, e_{9}, \ldots, e_{15+p} ; e_{4}, e_{5},-e_{2}+e_{6}-e_{7}\right\rangle .
\end{aligned}
$$

This shows that $B_{1}$ and $B_{2}$ are primitive sublattices with $B_{1}+B_{2}=$ $B_{1} \oplus B_{2}=M l(f)$, i.e. $r_{I}=1$.

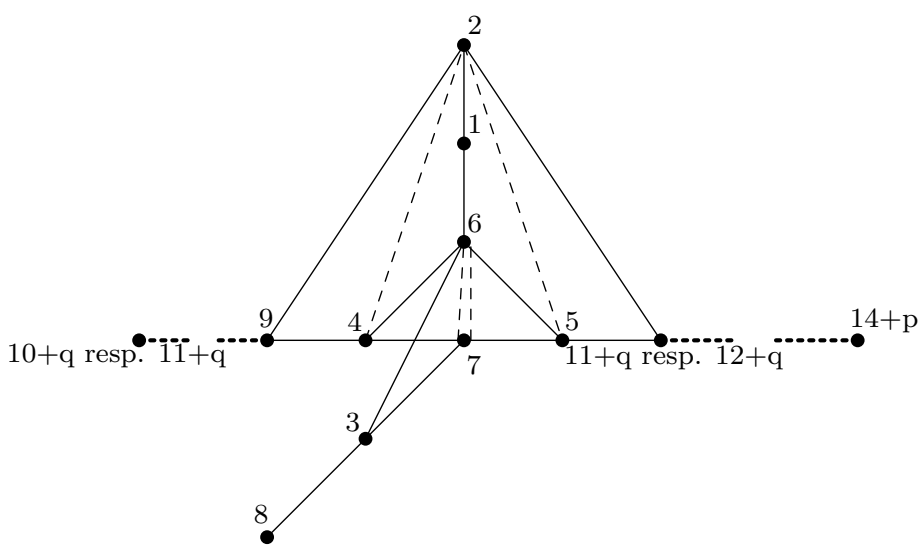

Figure 2. The CDD of a distinguished basis $e_{1}, \ldots, e_{\mu}$ for $S_{1,2 q-1}^{\sharp}$ resp. $S_{1,2 q}^{\sharp}$ from [Eb81, Tabelle $6 \&$ Abb. 16]

5.2. The series $S_{1, p}^{\sharp}$. Again we only describe the case when $p=2 q$ is even. But one can easily obtain the odd case $p=2 q-1$ from that via replacing each $e_{\alpha+q}$ by $e_{\alpha-1+q}$ in the following lists. The monodromy acts on the distinguished basis $e_{1}, \ldots, e_{\mu}$ with the CDD in figure 2 as 
follows:

$$
\begin{aligned}
e_{1} & \mapsto-e_{1}-e_{2}+e_{3}+e_{4}+e_{5}+e_{6}, \\
e_{2} & \mapsto e_{1}+e_{2}+e_{9}+e_{12+q}, \\
e_{3} & \mapsto-e_{1}-e_{6}+e_{7}+e_{8}, \\
e_{4} & \mapsto e_{2}-e_{6}+e_{7}+e_{9}, \\
e_{5} & \mapsto e_{2}-e_{6}+e_{7}+e_{12+q}, \\
e_{6} & \mapsto e_{1}-2 e_{2}+e_{3}+e_{4}+e_{5}+3 e_{6}-2 e_{7}, \\
e_{7} & \mapsto-2 e_{2}+e_{3}+e_{4}+e_{5}+2 e_{6}-e_{7}, \\
e_{8} & \mapsto-e_{3}-e_{8}, \\
e_{8+i} & \mapsto e_{9+i} \quad \text { for } 1 \leq i \leq 2+q, \\
e_{11+q} & \mapsto-e_{4}-e_{9}-e_{10}-\ldots-e_{11+q}, \\
e_{11+q+i} & \mapsto e_{12+q+i} \quad \text { for } 1 \leq i \leq 2+q, \\
e_{14+p} & \mapsto-e_{5}-e_{12+q}-e_{13+q}-\ldots-e_{14+p} .
\end{aligned}
$$

By table $(5.13)$ the generators of the Orlik blocks $B_{1}$ and $B_{2}$ are $\beta_{1}:=e_{8}$ and $\beta_{2}:=e_{9}$. The monodromy acts on them as follows:

$$
\begin{aligned}
e_{8} & \mapsto-e_{3}-e_{8} \mapsto e_{1}+e_{3}+e_{6}-e_{7} \\
& \mapsto-e_{1}-e_{2}+e_{3}+e_{4}+e_{5}+e_{6}+e_{8} \\
& \mapsto-e_{3}-e_{6}+e_{7} \mapsto-e_{8} \\
e_{9} & \mapsto e_{10} \mapsto \ldots \mapsto e_{11+q} \mapsto-e_{4}-e_{9}-e_{10}-\ldots-e_{11+q} \\
& \mapsto-e_{2}+e_{4}+e_{6}-e_{7} \mapsto-e_{12+q} \mapsto-e_{13+q} \mapsto \ldots \mapsto-e_{14+p} \\
& \mapsto e_{5}+e_{12+q}+\ldots+e_{14+p} \mapsto e_{2}-e_{5}-e_{6}+e_{7} \mapsto e_{9}
\end{aligned}
$$

Thus the characteristic polynomial of $M_{h}$ on $B_{j}$ is $b_{j}$, and the blocks are

$$
\begin{aligned}
& B_{1}=\left\langle e_{8}, e_{3}, e_{6}-e_{7}, e_{1},-e_{2}+e_{4}+e_{5}+e_{6}\right\rangle \\
& B_{2}=\left\langle e_{9}, e_{10}, \ldots, e_{14+p} ; e_{4}, e_{5},-e_{2}+e_{6}-e_{7}\right\rangle
\end{aligned}
$$

This shows that $B_{1}$ and $B_{2}$ are primitive sublattices with $B_{1}+B_{2}=$ $B_{1} \oplus B_{2}=M l(f)$ and $r_{I}=1$.

5.3. The series $U_{1, p}$. Here (and in all series except $W_{1, p}^{\sharp}$ and $S_{1, p}^{\sharp}$ ) the list of the monodromy action on the distinguished basis $e_{1}, \ldots, e_{\mu}$ with the CDD in figure 3 includes both cases $p=2 q$ and $p=2 q-1$. It 


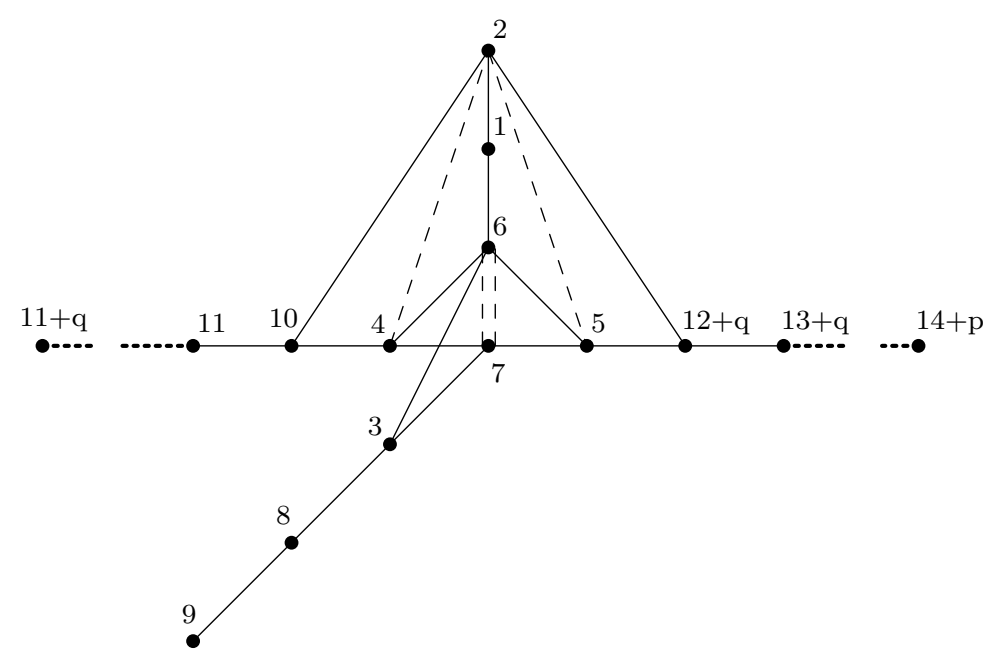

Figure 3. The CDD of a distinguished basis $e_{1}, \ldots, e_{\mu}$ for $U_{1, p}$ from [Eb81, Tabelle 6 \& Abb. 16]

looks as follows:

$$
\begin{aligned}
e_{1} & \mapsto-e_{1}-e_{2}+e_{3}+e_{4}+e_{5}+e_{6}, \\
e_{2} & \mapsto e_{1}+e_{2}+e_{10}+e_{12+q}, \\
e_{3} & \mapsto-e_{1}-e_{6}+e_{7}+e_{8}, \\
e_{4} & \mapsto e_{2}-e_{6}+e_{7}+e_{10}, \\
e_{5} & \mapsto e_{2}-e_{6}+e_{7}+e_{12+q}, \\
e_{6} & \mapsto e_{1}-2 e_{2}+e_{3}+e_{4}+e_{5}+3 e_{6}-2 e_{7}, \\
e_{7} & \mapsto-2 e_{1}+e_{3}+e_{4}+e_{5}+2 e_{6}-e_{7}, \\
e_{8} & \mapsto e_{9}, \\
e_{9} & \mapsto-e_{3}-e_{8}-e_{9}, \\
e_{9+i} & \mapsto e_{10+i} \quad \text { for } 1 \leq i \leq 1+q, \\
e_{11+q} & \mapsto-e_{4}-e_{10}-e_{11}-\ldots-e_{11+q}, \\
e_{11+q+i} & \mapsto e_{12+q+i} \quad \text { for } 1 \leq i \leq 2+p-q, \\
e_{14+p} & \mapsto-e_{5}-e_{12+q}-e_{13+q}-\ldots-e_{14+p} .
\end{aligned}
$$


By table (5.13) the generators of the Orlik blocks $B_{1}$ and $B_{2}$ are $\beta_{1}:=e_{8}$ and $\beta_{2}:=e_{10}$. The monodromy acts on them as follows:

$$
\begin{aligned}
e_{8} & \mapsto e_{9} \mapsto-e_{3}-e_{8}-e_{9} \mapsto e_{1}+e_{3}+e_{6}-e_{7} \\
& \mapsto-e_{1}-e_{2}+e_{3}+e_{4}+e_{5}+e_{6}+e_{8} \\
& \mapsto-e_{6}+e_{7}+e_{8}+e_{9} \mapsto-e_{1}-e_{3}-e_{6}+e_{7}-e_{8} \\
e_{10} & \mapsto e_{11} \mapsto \ldots \mapsto e_{11+q} \mapsto-e_{4}-e_{10}-e_{11}-\ldots-e_{11+q} \\
& \mapsto-e_{2}+e_{4}+e_{6}-e_{7} \mapsto-e_{12+q} \mapsto-e_{13+q} \mapsto \ldots \mapsto-e_{14+p} \\
& \mapsto e_{5}+e_{12+q}+\ldots+e_{14+p} \mapsto e_{2}-e_{5}-e_{6}+e_{7} \mapsto e_{10} .
\end{aligned}
$$

Thus the characteristic polynomial of $M_{h}$ on $B_{j}$ is $b_{j}$, and the blocks are

$$
\begin{aligned}
& B_{1}=\left\langle e_{1}, e_{3}, e_{8}, e_{9}, e_{6}-e_{7},-e_{2}+e_{4}+e_{5}+e_{6}\right\rangle, \\
& B_{2}=\left\langle e_{10}, e_{11}, \ldots, e_{14+p} ; e_{4}, e_{5},-e_{2}+e_{6}-e_{7}\right\rangle .
\end{aligned}
$$

Again $B_{1}$ and $B_{2}$ are primitive sublattices with $B_{1}+B_{2}=B_{1} \oplus B_{2}=$ $M l(f)$ and $r_{I}=1$.

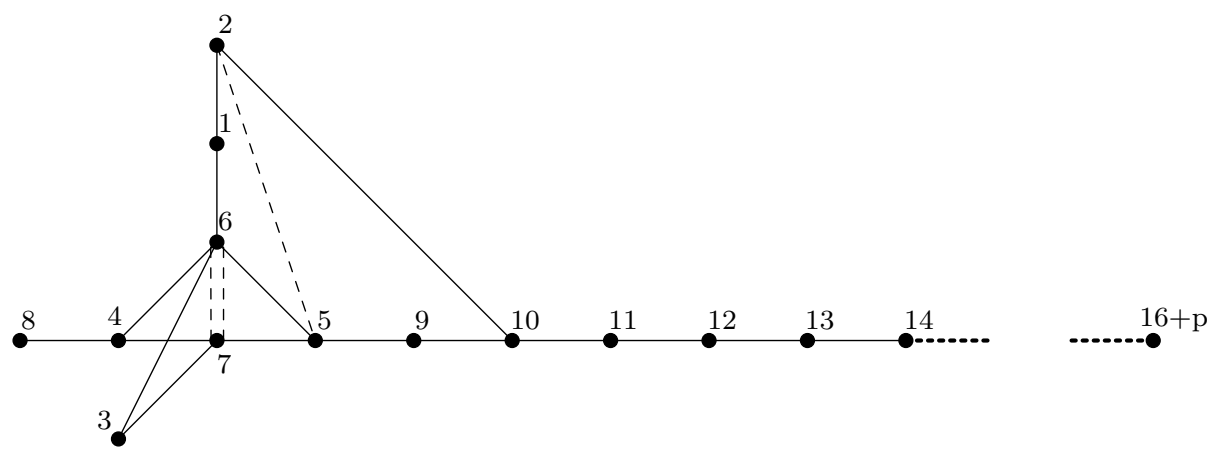

Figure 4. The CDD of a distinguished basis $e_{1}, \ldots, e_{\mu}$ for $E_{3, p}$ from [Eb81, Tabelle 6 \& Abb. 16] 
5.4. The series $E_{3, p}$. Here the monodromy acts on the distinguished basis $e_{1}, \ldots, e_{\mu}$ with the CDD in figure 4 as follows:

$$
\begin{aligned}
e_{1} & \mapsto e_{3}+e_{4}+e_{5}+e_{6}, \\
e_{2} & \mapsto e_{9}+e_{10}, \\
e_{3} & \mapsto-e_{1}-e_{3}-e_{6}+e_{7}, \\
e_{4} & \mapsto-e_{1}-e_{6}+e_{7}+e_{8}, \\
e_{5} & \mapsto-e_{1}-e_{6}+e_{7}+e_{9}, \\
e_{6} & \mapsto 2 e_{1}-e_{2}+e_{3}+e_{4}+e_{5}+3 e_{6}-2 e_{7}, \\
e_{7} & \mapsto e_{1}-e_{2}+e_{3}+e_{4}+e_{5}+2 e_{6}-e_{7}, \\
e_{8} & \mapsto-e_{4}-e_{8}, \\
e_{9} & \mapsto e_{1}+e_{2}+e_{10}, \\
e_{9+i} & \mapsto e_{10+i} \quad \text { for } 1 \leq i \leq 6+p, \\
e_{16+p} & \mapsto-e_{5}-e_{9}-e_{10}-\ldots-e_{16+p} .
\end{aligned}
$$

By table 5.13 the generators of the Orlik blocks $B_{1}$ and $B_{2}$ are $\beta_{1}:=e_{3}$ and $\beta_{2}:=e_{10}$. The monodromy acts on them as follows:

$$
\begin{aligned}
e_{3} & \mapsto-e_{1}-e_{3}-e_{6}+e_{7} \mapsto-e_{4}-e_{5}-e_{6} \\
& \mapsto e_{2}-e_{3}-e_{4}-e_{5}-e_{6}-e_{8}-e_{9} \mapsto-e_{5}-e_{7} \\
& \mapsto e_{2}-e_{3}-e_{4}-e_{5}-e_{6}-e_{9} \mapsto-e_{4}-e_{5}-e_{7}-e_{8} \\
& \mapsto e_{1}+e_{2}-e_{3}-e_{5}-e_{7}-e_{9} \mapsto e_{3}+e_{6}-e_{7} \\
& \mapsto-e_{3}, \\
e_{10} & \mapsto e_{11} \mapsto \ldots \mapsto e_{16+p} \mapsto-e_{5}-\sum_{i=9}^{16+p} e_{i} \\
& \mapsto-e_{2}+e_{5}+e_{6}-e_{7} \mapsto-e_{10} .
\end{aligned}
$$

Thus the characteristic polynomial of $M_{h}$ on $B_{j}$ is $b_{j}$, and the blocks are

$$
\begin{aligned}
& B_{1}=\left\langle e_{1}, e_{3}, e_{4}, e_{8}, e_{6}-e_{7}, e_{5}+e_{6}, e_{2}-e_{9}\right\rangle \\
& B_{2}=\left\langle e_{10}, e_{11}, \ldots, e_{16+p}, e_{5}+e_{9}, e_{2}-e_{6}+e_{7}+e_{9}\right\rangle
\end{aligned}
$$

This shows that $B_{1}$ and $B_{2}$ are primitive sublattices with $B_{1}+B_{2}=$ $B_{1} \oplus B_{2}$. Furthermore $B_{1} \oplus B_{2} \supset\left\{2 e_{2}\right\}$ and $B_{1}+B_{2}+\mathbb{Z} \cdot e_{2}=M l(f)$. This shows $\left[M l(f): B_{1} \oplus B_{2}\right]=2=r_{I}$. 


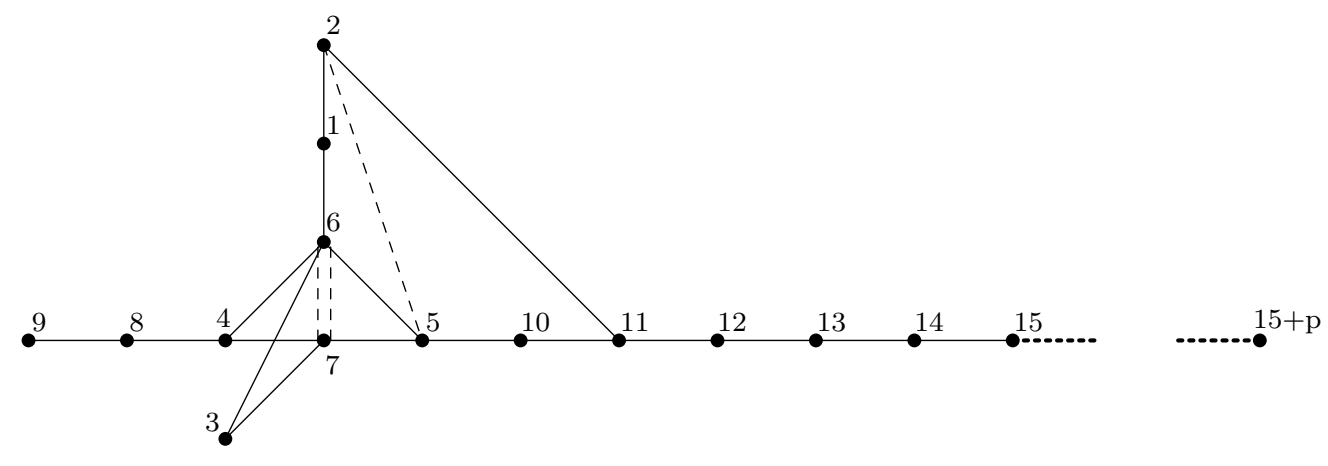

Figure 5. The CDD of a distinguished basis $e_{1}, \ldots, e_{\mu}$ for $Z_{1, p}$ from [Eb81, Tabelle 6 \& Abb. 16]

5.5. The series $Z_{1, p}$. Here the monodromy acts on the distinguished basis $e_{1}, \ldots, e_{\mu}$ with the CDD in figure 5 as follows:

$$
\begin{aligned}
e_{1} & \mapsto e_{3}+e_{4}+e_{5}+e_{6}, \\
e_{2} & \mapsto e_{10}+e_{11}, \\
e_{3} & \mapsto-e_{1}-e_{3}-e_{6}+e_{7}, \\
e_{4} & \mapsto-e_{1}-e_{6}+e_{7}+e_{8}, \\
e_{5} & \mapsto-e_{1}-e_{6}+e_{7}+e_{10}, \\
e_{6} & \mapsto 2 e_{1}-e_{2}+e_{3}+e_{4}+e_{5}+3 e_{6}-2 e_{7}, \\
e_{7} & \mapsto e_{1}-e_{2}+e_{3}+e_{4}+e_{5}+2 e_{6}-e_{7}, \\
e_{8} & \mapsto e_{9}, \\
e_{9} & \mapsto-e_{4}-e_{8}-e_{9}, \\
e_{10} & \mapsto e_{1}+e_{2}+e_{11}, \\
e_{11+i} & \mapsto e_{12+i} \quad \text { for } 1 \leq i \leq 3+p, \\
e_{15+p} & \mapsto-e_{5}-e_{10}-e_{11}-\ldots-e_{15+p} .
\end{aligned}
$$


Here there are three Orlik blocks $B_{1}, B_{2}$ and $B_{3}$. By table 5.13 their generators are $\beta_{1}:=e_{8}, \beta_{2}:=e_{11}$ and $\beta_{3}:=e_{3}+e_{4}-e_{9}$. The monodromy acts on them as follows:

$$
\begin{aligned}
e_{8} & \mapsto e_{9} \mapsto-e_{4}-e_{8}-e_{9} \mapsto e_{1}+e_{4}+e_{6}-e_{7} \\
& \mapsto e_{3}+e_{4}+e_{5}+e_{6}+e_{8} \\
& \mapsto-e_{1}-e_{2}+e_{4}+e_{5}+e_{7}+e_{8}+e_{9}+e_{10} \\
& \mapsto-e_{4}-e_{6}+e_{7} \mapsto-e_{8} \\
e_{11} & \mapsto e_{12} \mapsto \ldots \mapsto e_{15+p} \mapsto-e_{5}-\sum_{i=10}^{15+p} e_{i} \\
& \mapsto-e_{2}+e_{5}+e_{6}-e_{7} \mapsto-e_{11} \\
e_{3}-e_{4}-e_{9} & \mapsto-e_{3}+e_{4}+e_{9} .
\end{aligned}
$$

Thus the characteristic polynomial of $M_{h}$ on $B_{j}$ is $b_{j}$, and the blocks are

$$
\begin{aligned}
B_{1}= & \left\langle e_{8}, e_{9}, e_{4}, e_{1}, e_{6}-e_{7}, e_{3}+e_{5}+e_{6}\right. \\
& \left.-e_{2}+e_{5}+e_{7}+e_{10}\right\rangle \\
B_{2}= & \left\langle e_{11}, e_{12}, \ldots, e_{15+p} ; e_{5}+e_{10},-e_{2}+e_{5}+e_{6}-e_{7}\right\rangle \\
B_{3}= & \left\langle e_{3}-e_{4}-e_{9}\right\rangle
\end{aligned}
$$

This shows that $B_{1}, B_{2}$ and $B_{3}$ are primitive sublattices with $B_{1}+$ $B_{2}+B_{3}=B_{1} \oplus B_{2} \oplus B_{3}$. Furthermore $B_{1} \oplus B_{2} \oplus B_{3} \supset\left\{2 e_{5}\right\}$ and $B_{1}+B_{2}+B_{3}+\mathbb{Z} \cdot e_{5}=M l(f)$. This shows $\left[M l(f): B_{1} \oplus B_{2} \oplus B_{3}\right]=$ $2=r_{I}$. 


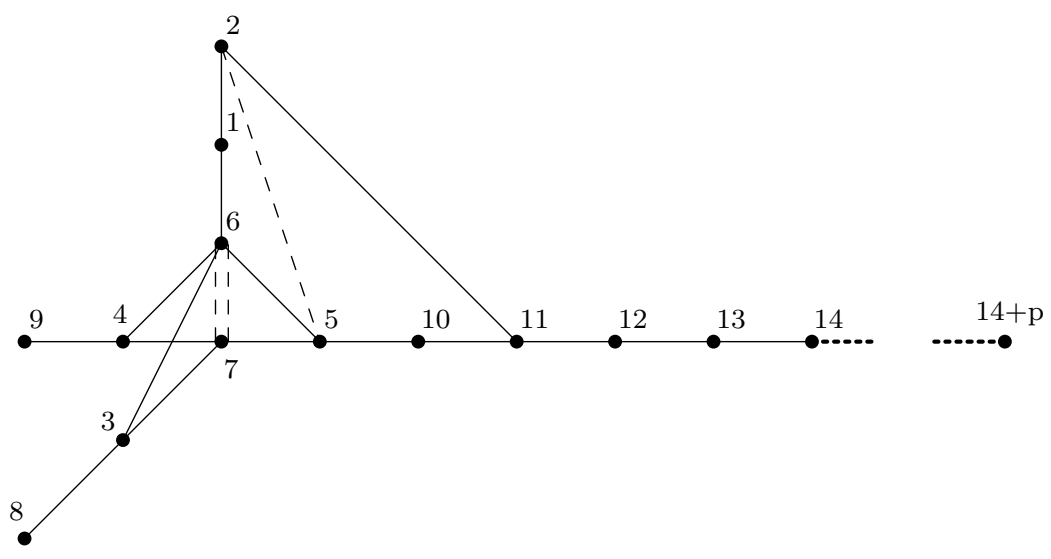

Figure 6. The CDD of a distinguished basis $e_{1}, \ldots, e_{\mu}$ for $Q_{2, p}$ from [Eb81, Tabelle 6 \& Abb. 16]

5.6. The series $Q_{2, p}$. Here the monodromy acts on the distinguished basis $e_{1}, \ldots, e_{\mu}$ with the CDD in figure 6 as follows:

$$
\begin{aligned}
e_{1} & \mapsto e_{3}+e_{4}+e_{5}+e_{6}, \\
e_{2} & \mapsto e_{10}+e_{11}, \\
e_{3} & \mapsto-e_{1}-e_{6}+e_{7}+e_{8}, \\
e_{4} & \mapsto-e_{1}-e_{6}+e_{7}+e_{9}, \\
e_{5} & \mapsto-e_{1}-e_{6}+e_{7}+e_{10}, \\
e_{6} & \mapsto 2 e_{1}-e_{2}+e_{3}+e_{4}+e_{5}+3 e_{6}-2 e_{7}, \\
e_{7} & \mapsto e_{1}-e_{2}+e_{3}+e_{4}+e_{5}+2 e_{6}-e_{7}, \\
e_{8} & \mapsto-e_{3}-e_{8}, \\
e_{9} & \mapsto-e_{4}-e_{9}, \\
e_{10} & \mapsto e_{1}+e_{2}+e_{11}, \\
e_{10+i} & \mapsto e_{11+i} \quad \text { for } 1 \leq i \leq 3+p, \\
e_{14+p} & \mapsto-e_{5}-e_{10}-e_{11}-\ldots-e_{14+p} .
\end{aligned}
$$


By table $(5.13)$ the generators of the Orlik blocks $B_{1}$ and $B_{2}$ are $\beta_{1}:=e_{8}$ and $\beta_{2}:=e_{11}$. The monodromy acts on them as follows:

$$
\begin{aligned}
e_{8} & \mapsto-e_{3}-e_{8} \mapsto e_{1}+e_{3}+e_{6}-e_{7} \mapsto e_{3}+e_{4}+e_{5}+e_{6}+e_{8} \\
& \mapsto-e_{1}-e_{2}+e_{4}+e_{5}+e_{7}+e_{9}+e_{10} \mapsto-e_{4}-e_{6}+e_{7} \\
& \mapsto-e_{9} \mapsto e_{4}+e_{9} \mapsto-e_{1}-e_{4}-e_{6}+e_{7}, \\
e_{11} & \mapsto e_{12} \mapsto \ldots \mapsto e_{14+p} \mapsto-e_{5}-\sum_{i=10}^{14+p} e_{i} \\
& \mapsto-e_{2}+e_{5}+e_{6}-e_{7} \mapsto-e_{11} .
\end{aligned}
$$

Thus the characteristic polynomial of $M_{h}$ on $B_{j}$ is $b_{j}$, and the blocks are

$$
\begin{aligned}
B_{1}= & \left\langle e_{8}, e_{3}, e_{9}, e_{4}, e_{1}, e_{6}-e_{7}\right. \\
& \left.e_{5}+e_{6},-e_{2}+e_{5}+e_{7}+e_{10}\right\rangle \\
B_{2}= & \left\langle e_{11}, e_{12}, \ldots, e_{14+p} ; e_{5}+e_{10},-e_{2}+e_{5}+e_{6}-e_{7}\right\rangle
\end{aligned}
$$

This shows that $B_{1}$ and $B_{2}$ are primitive sublattices with $B_{1}+B_{2}=$ $B_{1} \oplus B_{2}$. Furthermore $B_{1} \oplus B_{2} \supset\left\{2 e_{5}\right\}$ and $B_{1}+B_{2}+\mathbb{Z} \cdot e_{5}=\operatorname{Ml}(f)$. This shows $\left[M l(f): B_{1} \oplus B_{2}\right]=2=r_{I}$.

The proof of (5.4) for $Q_{2, p}$ was postponed to this subsection and has to be given now. Recall the definition (5.37) of $b_{4}$ and recall $b_{4}=\Phi_{4}$ for $Q_{2,4 s}$ and $b_{4}=1$ for the other $Q_{2, p}$. The next aims are:

(i) For $Q_{2,4 s}$ : To show for any $g \in G_{\mathbb{Z}} \cup \operatorname{Aut}\left(B_{1} \oplus B_{2}, L\right)$

$$
g:\left(B_{1}\right)_{b_{4}} \rightarrow\left(B_{1}\right)_{b_{4}} \text { and }\left(B_{2}\right)_{b_{4}} \rightarrow\left(B_{2}\right)_{b_{4}} .
$$

(ii) For all $Q_{2, p}$ : To find an element $\gamma_{4} \in\left(B_{1}\right)_{\Phi_{4}}$ with

$$
\begin{aligned}
B_{1} \oplus B_{2} & =\left\{a \in M l(f) \mid L\left(a, \gamma_{4}\right) \equiv 0(2)\right\} \\
& =\left\{a \in M l(f) \mid L\left(a, M_{h}\left(\gamma_{4}\right)\right) \equiv 0(2)\right\} \\
g\left(\gamma_{4}\right) & \in\left\{ \pm \gamma_{4}, \pm M_{h}\left(\gamma_{4}\right)\right\} \quad \text { for any } g \in G_{\mathbb{Z}}
\end{aligned}
$$

(iii) For all $Q_{2, p}$ : To find an element $\gamma_{5} \in M l(f)$ with

$$
B_{1}+B_{2}+\mathbb{Z} \cdot \gamma_{5}=M l(f)
$$

and $g\left(\gamma_{5}\right) \in M l(f)$ for any $g \in \operatorname{Aut}\left(B_{1} \oplus B_{2}, L\right)$.

For all $Q_{2, p}$ define

$$
\begin{aligned}
\gamma_{1} & :=\frac{b_{1}}{\Phi_{4}}\left(M_{h}\right)\left(\beta_{1}\right)=\left(\Phi_{12} \Phi_{3}\right)\left(M_{h}\right)\left(e_{8}\right) \\
& =\left(t^{6}+t^{5}-t^{3}+t+1\right)\left(M_{h}\right)\left(e_{8}\right) \\
& =-2 e_{3}-2 e_{4}-e_{5}-2 e_{6}+e_{7}-e_{8}-e_{9} .
\end{aligned}
$$


Obviously $M_{h}^{2}\left(\gamma_{1}\right)=-\gamma_{1}$. By remark 2.6 $(\mathrm{v}),\left(B_{1}\right)_{\Phi_{4}}$ is an Orlik block with cyclic generator $\gamma_{1}$, so $\left(B_{1}\right)_{\Phi_{4}}=\mathbb{Z} \cdot \gamma_{1} \oplus \mathbb{Z} \cdot M_{h}\left(\gamma_{1}\right)$. Calculate

$$
M_{h}\left(\gamma_{1}\right)=2 e_{1}+e_{2}-e_{5}+e_{6}-2 e_{7}-e_{8}-e_{9}-e_{10} .
$$

For $Q_{2,4 s}$ define

$$
\begin{aligned}
\gamma_{2} & :=\frac{b_{2}}{\Phi_{4}}\left(M_{h}\right)\left(\beta_{2}\right)=\frac{t^{6+4 s}+1}{t^{2}+1}\left(M_{h}\right)\left(e_{11}\right) \\
& =\left(t^{4+4 s}-t^{2+4 s}+t^{4 s}-\ldots-t^{2}+1\right)\left(M_{h}\right)\left(e_{11}\right) \\
& =-e_{5}-e_{10}+(-1) \sum_{j=1}^{2+2 s} e_{10+2 j}+(-2) \sum_{j=1}^{1+s} e_{9+4 j} .
\end{aligned}
$$

Obviously $M_{h}^{2}\left(\gamma_{2}\right)=-\gamma_{2}$. By remark 2.6 $(\mathrm{v}),\left(B_{2}\right)_{\Phi_{4}}$ is an Orlik block with cyclic generator $\gamma_{2}$, so $\left(B_{2}\right)_{\Phi_{4}}=\mathbb{Z} \cdot \gamma_{2} \oplus \mathbb{Z} \cdot M_{h}\left(\gamma_{2}\right)$. Calculate

$$
M_{h}\left(\gamma_{2}\right)=-e_{2}+e_{5}+e_{6}-e_{7}+\sum_{j=1}^{2+2 s}(-1)^{j+1} e_{10+2 j}
$$

For $Q_{2,4 s}$ define

$$
\gamma_{3}:=\frac{1}{2}\left(\gamma_{1}+M_{h}\left(\gamma_{1}\right)+\gamma_{2}+M_{h}\left(\gamma_{2}\right)\right)
$$

and observe

$$
\begin{aligned}
\gamma_{3} & =e_{1}-\sum_{j \in\{3,4,5,7,8,9,10\}} e_{j}-\sum_{j=1}^{1+s}\left(e_{9+4 j}+e_{10+4 j}\right) \\
& \stackrel{!}{\in} \operatorname{Ml}(f) .
\end{aligned}
$$

Together with $\left[M l(f): B_{1} \oplus B_{2}\right]=2$ this shows (5.120) and that $\gamma_{1}, M_{h}\left(\gamma_{1}\right), \gamma_{3}, M_{h}\left(\gamma_{3}\right)$ is a $\mathbb{Z}$-basis of $M l(f)_{\Phi_{4}}$. We want to calculate the matrices of $L$ with respect to the basis $\gamma_{1}, M_{h}\left(\gamma_{1}\right), \gamma_{2}, M_{h}\left(\gamma_{2}\right)$ of $\left(B_{1} \oplus B_{2}\right)_{\Phi_{4}}$ and the basis $\gamma_{1}, M_{h}\left(\gamma_{1}\right), \gamma_{3}, M_{h}\left(\gamma_{3}\right)$ of $M l(f)_{\Phi_{4}}$. Essentially we need to calculate only the values $L\left(\gamma_{1}, \gamma_{1}\right)$ and $L\left(\gamma_{2}, \gamma_{2}\right)$, because of (5.3) and because of the identities for any $a \in M l(f)_{\Phi_{4}}$,

$$
\begin{aligned}
L\left(a, M_{h}(a)\right) & =L\left(M_{h}(a), M_{h}^{2}(a)\right)=-L\left(M_{h}(a), a\right) \\
& =L(a, a)=L\left(M_{h}(a), M_{h}(a)\right) .
\end{aligned}
$$

Using $M_{h}^{2}\left(\gamma_{j}\right)=-\gamma_{j}$ and calculations similar to (2.17), we find

$$
\begin{aligned}
L\left(\gamma_{1}, \gamma_{1}\right) & =L\left(\frac{b_{1}}{\Phi_{4}}\left(-M_{h}^{-1}\right)\left(\gamma_{1}\right), e_{8}\right)=3 \cdot L\left(M_{h}\left(\gamma_{1}\right), e_{8}\right)=3, \\
L\left(\gamma_{2}, \gamma_{2}\right) & =L\left(\frac{b_{2}}{\Phi_{4}}\left(M_{h}^{-1}\right)\left(\gamma_{2}\right), e_{11}\right) \\
& =(3+2 s) \cdot L\left(\gamma_{2}, e_{11}\right)=3+2 s,
\end{aligned}
$$


thus

$$
L\left(\left(\begin{array}{c}
\gamma_{1} \\
M_{h}\left(\gamma_{1}\right) \\
\gamma_{2} \\
M_{h}\left(\gamma_{2}\right)
\end{array}\right),\left(\begin{array}{c}
\gamma_{1} \\
M_{h}\left(\gamma_{1}\right) \\
\gamma_{2} \\
M_{h}\left(\gamma_{2}\right)
\end{array}\right)^{t}\right)=\left(\begin{array}{cccc}
3 & 3 & 0 & 0 \\
-3 & 3 & 0 & 0 \\
0 & 0 & 3+2 s & 3+2 s \\
0 & 0 & -(3+2 s) & 3+2 s
\end{array}\right)
$$

and

$$
L\left(\left(\begin{array}{c}
\gamma_{1} \\
M_{h}\left(\gamma_{1}\right) \\
\gamma_{3} \\
M_{h}\left(\gamma_{3}\right)
\end{array}\right),\left(\begin{array}{c}
\gamma_{1} \\
M_{h}\left(\gamma_{1}\right) \\
\gamma_{3} \\
M_{h}\left(\gamma_{3}\right)
\end{array}\right)^{t}\right)=\left(\begin{array}{cccc}
3 & 3 & 3 & 0 \\
-3 & 3 & 0 & 3 \\
0 & 3 & 3+s & 3+s \\
-3 & 0 & -(3+s) & 3+s
\end{array}\right)
$$

The quadratic form associated to the last matrix is

$$
\begin{aligned}
\frac{3}{2} \cdot\left[\left(x_{1}+x_{3}\right)^{2}+\left(x_{1}-x_{4}\right)^{2}+\left(x_{2}+x_{3}\right)^{2}\right. & \left.+\left(x_{2}+x_{4}\right)^{2}\right] \\
& +s \cdot\left(x_{3}^{2}+x_{4}^{2}\right) .
\end{aligned}
$$

This shows (first for $Q_{2,4 s}$, but in fact for all $Q_{2, p}$ )

$$
\left\{a \in M l(f)_{\Phi_{4}} \mid L(a, a)=3\right\}=\left\{ \pm \gamma_{1}, \pm M_{h}\left(\gamma_{1}\right)\right\}
$$

and because of $\left(B_{1} \oplus B_{2}\right)_{\Phi_{4}} \subset M l(f)_{\Phi_{4}}$

$$
\left\{a \in\left(B_{1} \oplus B_{2}\right)_{\Phi_{4}} \mid L(a, a)=3\right\}=\left\{ \pm \gamma_{1}, \pm M_{h}\left(\gamma_{1}\right)\right\},
$$

This implies that any $g \in G_{\mathbb{Z}} \cup \operatorname{Aut}\left(B_{1} \oplus B_{2}, L\right)$ maps the set $\left\{ \pm \gamma_{1}, \pm M_{h}\left(\gamma_{1}\right)\right\}$ to itself and thus $\left(B_{1}\right)_{\Phi_{4}}$ to itself and thus the $L$ orthogonal sublattice $\left(B_{2}\right)_{\Phi_{4}}$ to itself. This shows (5.117) and gives (i).

Define for all $Q_{2, p}$

$$
\begin{aligned}
\gamma_{4} & :=\gamma_{1}+M_{h}\left(\gamma_{1}\right) \\
& =2 e_{1}+e_{2}-2 e_{3}-2 e_{4}-2 e_{5}-e_{6}-e_{7}-2 e_{8}-2 e_{9}-e_{10}
\end{aligned}
$$

Observe

$$
\begin{aligned}
M_{h}\left(\gamma_{4}\right) & =-\gamma_{1}+M_{h}\left(\gamma_{1}\right) \\
& =-2 \gamma_{1}+\gamma_{4} .
\end{aligned}
$$

(5.134) and (5.137) imply (5.119) . (5.138) implies the second equality in (5.118). One calculates

$$
L\left(e_{8}, \gamma_{4}\right)=0 .
$$

This shows $L\left(e_{8}, M_{h}\left(\gamma_{4}\right)\right) \equiv 0(2)$ (in fact, it is $=-2$ ). The $M_{h^{-}}$ invariance of $L$ and the fact that $e_{8}$ is a cyclic generator of the Orlik block $B_{1}$ give $B_{1} \subset\left\{a \in M l(f) \mid L\left(a, \gamma_{4}\right) \equiv 0(2)\right\}$. As (5.3) implies $L\left(B_{2}, \gamma_{4}\right)=0$, so $B_{1} \oplus B_{2} \subset\left\{a \in M l(f) \mid L\left(a, \gamma_{4}\right) \equiv 0(2)\right\}$. Now 
$r_{I}=2$ and for example $L\left(e_{2}, \gamma_{4}\right)=-1 \not \equiv 0(2)$ show (5.118) and (ii). (ii) implies $G_{\mathbb{Z}} \subset \operatorname{Aut}\left(B_{1} \oplus B_{2}, L\right)$.

(iii) implies $\operatorname{Aut}\left(B_{1} \oplus B_{2}, L\right) \subset G_{\mathbb{Z}}$, but (iii) has still to be proved.

We continue as in the final part of the proof of part (a) for the other series. (i) holds. Lemma 2.8 can be applied. Therefore (5.42) and (5.43) hold for $Q_{2, p}$. The group $\operatorname{Aut}\left(B_{1} \oplus B_{2}, L\right)$ for $12 \not p$ is generated by $M_{h},-\mathrm{id},\left.M_{h}\right|_{B_{1}} \times\left.\mathrm{id}\right|_{B_{2}}$ and ( $\left.-\mathrm{id}\right)\left.\right|_{B_{1}} \times\left.\mathrm{id}\right|_{B_{2}}$, and analogously for the group in (5.43) if $12 \mid p$.

For $Q_{2,4 s}$ we define $\gamma_{5}:=\gamma_{3}$. It satisfies (5.120). If $12 \mid 4 s$, it is in $\left(B_{1}\right)_{b_{1} / \Phi_{m}}+\left(B_{3}\right)_{b_{2} / \Phi_{m}}$, so we can work with the group in (5.43). If $12 \bigwedge 4 s$, we work with the group in (5.42). In both cases $\gamma_{5}$ satisfies (5.121), because of

$$
\begin{aligned}
\left(\left.M_{h}\right|_{B_{1}} \times\left.\mathrm{id}\right|_{B_{2}}\right)\left(\gamma_{5}\right) & =\gamma_{5}-M_{h}\left(\gamma_{1}\right) \in M l(f) \\
\left(\left.(-\mathrm{id})\right|_{B_{1}} \times\left.\mathrm{id}\right|_{B_{2}}\right)\left(\gamma_{5}\right) & =\gamma_{5}-\left(\gamma_{1}+M_{h}\left(\gamma_{1}\right)\right) \in M l(f) .
\end{aligned}
$$

For other $Q_{2, p}$, we choose a different (rather simple) $\gamma_{5}$,

$$
\begin{aligned}
\gamma_{5}:= & e_{10} \\
= & \frac{1}{2}\left(-e_{2}+e_{6}-e_{7}+e_{10}\right)-\frac{1}{2}\left(-e_{2}+e_{6}-e_{7}-e_{10}\right), \\
\text { with } \quad & -e_{2}+e_{6}-e_{7}+e_{10} \in B_{1}, \quad-e_{2}+e_{6}-e_{7}-e_{10} \in B_{2} .
\end{aligned}
$$

Then 5.120 holds. And

$$
\begin{aligned}
\left(\left.M_{h}\right|_{B_{1}} \times\left.\mathrm{id}\right|_{B_{2}}\right)\left(\gamma_{5}\right) & =e_{1}+e_{2} \in M l(f), \\
\left(\left.(-\mathrm{id})\right|_{B_{1}} \times\left.\mathrm{id}\right|_{B_{2}}\right)\left(\gamma_{5}\right) & =e_{2}-e_{6}+e_{7} \in M l(f) .
\end{aligned}
$$

In any case (5.120) and (5.121) and (iii) hold. Thus Aut $\left(B_{1} \oplus B_{2}, L\right) \subset$ $G_{\mathbb{Z}}$, and $(5.4)$ is proved for $Q_{2, p}$. 


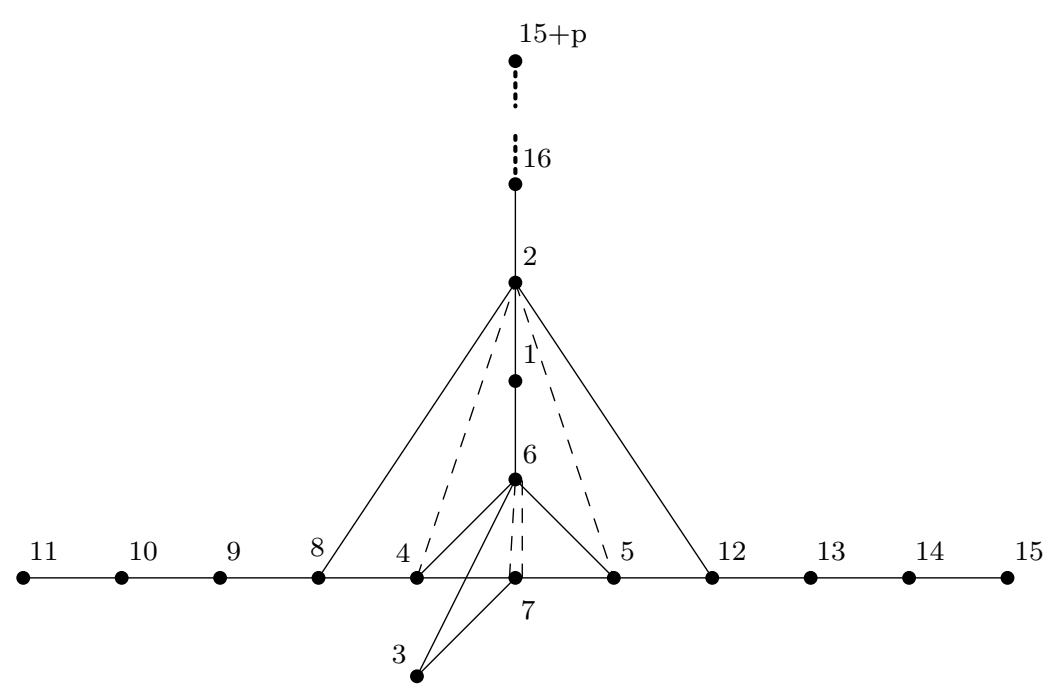

Figure 7. The CDD of a distinguished basis $e_{1}, \ldots, e_{\mu}$ for $W_{1, p}$ from [Eb81, Tabelle $6 \&$ Abb. 16]

5.7. The series $W_{1, p}$. Here the monodromy acts on the distinguished basis $e_{1}, \ldots, e_{\mu}$ with the CDD in figure 7 as follows:

$$
\begin{aligned}
e_{1} & \mapsto-e_{1}-e_{2}+e_{3}+e_{4}+e_{5}+e_{6}, \\
e_{2} & \mapsto 2 e_{1}+2 e_{2}+e_{8}+e_{12}+e_{16}, \\
e_{3} & \mapsto-e_{1}-e_{3}-e_{6}+e_{7}, \\
e_{4} & \mapsto e_{2}-e_{6}+e_{7}+e_{8}, \\
e_{5} & \mapsto e_{2}-e_{6}+e_{7}+e_{12}, \\
e_{6} & \mapsto e_{1}-2 e_{2}+e_{3}+e_{4}+e_{5}+3 e_{6}-2 e_{7}, \\
e_{7} & \mapsto-2 e_{2}+e_{3}+e_{4}+e_{5}+2 e_{6}-e_{7}, \\
e_{8} & \mapsto e_{9}, \\
e_{9} & \mapsto e_{10}, \\
e_{10} & \mapsto e_{11}, \\
e_{11} & \mapsto-e_{4}-e_{8}-e_{9}-e_{10}-e_{11}, \\
e_{12} & \mapsto e_{13}, \\
e_{13} & \mapsto e_{14}, \\
e_{14} & \mapsto e_{15}, \\
e_{15} & \mapsto-e_{5}-e_{12}-e_{13}-e_{14}-e_{15}, \\
e_{15+i} & \mapsto e_{16+i} \quad \text { for } 1 \leq i \leq p-1, \\
e_{15+p} & \mapsto-e_{1}-e_{2}-e_{16}-e_{17}-\ldots-e_{15+p} .
\end{aligned}
$$


By table (5.13) the generators of the Orlik blocks $B_{1}$ and $B_{2}$ are $\beta_{1}:=$ $e_{3}+e_{9}+e_{11}$ and $\beta_{2}:=e_{16}$. The monodromy acts on them as follows:

$$
\begin{aligned}
& e_{3}+e_{9}+e_{11} \mapsto-e_{1}-e_{3}-e_{4}-e_{6}+e_{7}-e_{8}-e_{9}-e_{11} \\
& \mapsto e_{1}-e_{5}-e_{7}+e_{11} \\
& \mapsto-e_{1}-e_{4}-e_{8}-e_{9}-e_{10}-e_{11}-e_{12} \\
& \mapsto e_{1}-e_{3}-e_{5}-e_{7}-e_{13} \\
& \mapsto e_{3}+e_{6}-e_{7}-e_{12}-e_{14} \\
& \mapsto \quad-e_{3}-e_{13}-e_{15} \\
& \mapsto e_{1}+e_{3}+e_{5}+e_{6}-e_{7}+e_{12}+e_{13}+e_{15} \\
& \mapsto-e_{1}+e_{4}+e_{7}-e_{15} \\
& \mapsto e_{1}+e_{5}+e_{8}+e_{12}+e_{13}+e_{14}+e_{15} \\
& \mapsto-e_{1}+e_{3}+e_{4}+e_{7}+e_{9} \\
& \mapsto-e_{3}-e_{6}+e_{7}+e_{8}+e_{10} \\
& \mapsto e_{3}+e_{9}+e_{11} \text {, } \\
& e_{16} \mapsto e_{17} \mapsto \ldots \mapsto e_{14+p} \mapsto e_{15+p} \\
& \mapsto-e_{1}-e_{2}-\sum_{i=16}^{15+p} e_{i} \\
& \mapsto-e_{3}-e_{4}-e_{5}-e_{6}-e_{8}-e_{12} \\
& \mapsto-e_{4}-e_{5}-e_{7}-e_{8}-e_{9}-e_{12}-e_{13} \\
& \mapsto-e_{3}-e_{4}-e_{5}-e_{7}-e_{8}-e_{9}-e_{10}-e_{12}-e_{13}-e_{14} \\
& \mapsto e_{1}-e_{4}-e_{5}+e_{6}-2 e_{7}-\sum_{i=8}^{15} e_{i} \\
& \mapsto \quad-e_{2}+e_{4}+e_{5}+2 e_{6}-2 e_{7} \\
& \mapsto-e_{16} \text {. }
\end{aligned}
$$

Thus the characteristic polynomial of $M_{h}$ on $B_{j}$ is $b_{j}$. Here the blocks $B_{1}$ and $B_{2}$ are generated by the first $\operatorname{deg} b_{1}$ respectively $\operatorname{deg} b_{2}$ of the elements above. Here $B_{1}+B_{2}=B_{1} \oplus B_{2}$ and $\left[M l(f): B_{1} \oplus B_{2}\right]=2=$ $r_{I}$ follow by the calculation of the determinant which expresses these generators of $B_{1}$ and $B_{2}$ in the distinguished basis $e_{1}, \ldots, e_{\mu}$. Then it also follows that $B_{1}$ and $B_{2}$ are primitive sublattices.

The proof of (5.4) for $W_{1,6 s-3}$ was postponed to this subsection and has to be given here. But the majority of the arguments was already given in the proof of part (a). It rests to prove the following two points:

(i) (5.34) holds for $W_{1,3}$. 
(ii) In the case $W_{1,6 s-3}$, any $g \in G_{\mathbb{Z}} \cup \operatorname{Aut}\left(B_{1} \oplus B_{2}, L\right)$ maps $\left(B_{1}\right)_{b_{4}}$ to itself and $\left(B_{2}\right)_{b_{4}}$ to itself. Here $b_{4}=\Phi_{6} \Phi_{2}$.

For the rest of this subsection we restrict to $W_{6 s-3}$. Define for it

$$
\begin{aligned}
\delta_{1} & :=\frac{b_{1}}{\Phi_{6} \Phi_{2}}\left(M_{h}\right)\left(\beta_{1}\right)=\left(\Phi_{12} \Phi_{3}\right)\left(M_{h}\right)\left(e_{3}+e_{9}+e_{11}\right) \\
& =\Phi_{3}\left(M_{h}\right)\left(e_{9}-e_{13}\right)=e_{9}+e_{10}+e_{11}-e_{13}-e_{14}-e_{15} \\
\delta_{2} & :=\frac{b_{2}}{\Phi_{6} \Phi_{2}}\left(M_{h}\right)\left(\beta_{2}\right)=\frac{t^{6+p}+1}{t^{3}+1}\left(M_{h}\right)\left(e_{16}\right) \\
& =\left(t^{3+p}-t^{p}+\ldots-t^{3}+1\right)\left(M_{h}\right)\left(e_{16}\right) \\
& =e_{1}+e_{2}-\sum_{j \in\{3,4,5,7,8,9,10,12,13,14\}} e_{j}+\sum_{j=1}^{p} e_{15+j}+\sum_{j=0}^{p / 3-1}(-1)^{j} e_{16+3 j} .
\end{aligned}
$$

$\delta_{1}$ and $\delta_{2}$ are cyclic generators of the Orlik blocks $\left(B_{1}\right)_{\Phi_{6} \Phi_{2}}$ and $\left(B_{2}\right)_{\Phi_{6} \Phi_{2}}$, see remark 2.6 $(\mathrm{v})$. Thus $\delta_{i}, M_{h}\left(\delta_{i}\right)$ and $M_{h}^{2}\left(\delta_{i}\right)$ are a $\mathbb{Z}$-basis of $\left(B_{i}\right)_{\Phi_{6} \Phi_{2}}$. One calculates

$$
\begin{aligned}
M_{h}\left(\delta_{1}\right) & =-e_{4}-e_{8}-e_{9}+e_{5}+e_{12}+e_{13} \\
M_{h}^{2}\left(\delta_{1}\right) & =-e_{8}-e_{9}-e_{10}+e_{12}+e_{13}+e_{14} \\
M_{h}\left(\delta_{2}\right) & =e_{1}+e_{3}+2 e_{6}-2 e_{7}-\sum_{j=9,10,11,13,14,15} e_{j} \\
& +\sum_{j=0}^{p / 3-1}(-1)^{j} e_{17+3 j} \\
M_{h}^{2}\left(\delta_{2}\right) & =-e_{2}+2 e_{4}+2 e_{5}+2 e_{6}-e_{7}+e_{8}+e_{9}+e_{12}+e_{13} \\
& +\sum_{j=0}^{p / 3-1}(-1)^{j} e_{18+3 j} .
\end{aligned}
$$

We need to calculate the $6 \times 6$ matrix of values of $L$ for the $\mathbb{Z}$ basis $\delta_{1}, M_{h}\left(\delta_{1}\right), M_{h}^{2}\left(\delta_{1}\right), \delta_{2}, M_{h}\left(\delta_{2}\right), M_{h}^{2}\left(\delta_{2}\right)$ of $\left(B_{1} \oplus B_{2}\right)_{\Phi_{6} \Phi_{2}}$. Because of (5.3), it is block diagonal with two $3 \times 3$ blocks. Because $L$ is $M_{h^{-}}$ invariant and because of the identities for any $a \in M l(f)_{\Phi_{6} \Phi_{2}}$,

$$
\begin{gathered}
L\left(M_{h}(a), a\right)=-L(a, a), \quad L\left(M_{h}^{2}(a), a\right)=-L\left(a, M_{h}(a)\right), \\
L\left(a, M_{h}^{2}(a)\right)=L\left(M_{h}(a), M_{h}^{3}(a)\right)=-L\left(M_{h}(a), a\right)=L(a, a),
\end{gathered}
$$


each $3 \times 3$ matrix is determined by two values. The matrices are

$$
\begin{array}{r}
L\left(M_{h}^{i}\left(\delta_{1}\right), M_{h}^{j}\left(\delta_{1}\right)\right)_{i, j=0,1,2}=\left(\begin{array}{ccc}
2 & 2 & 2 \\
-2 & 2 & 2 \\
-2 & -2 & 2
\end{array}\right), \\
L\left(M_{h}^{i}\left(\delta_{2}\right), M_{h}^{j}\left(\delta_{2}\right)\right)_{i, j=0,1,2}=\left(\begin{array}{ccc}
1+2 s & 0 & 1+2 s \\
-1-2 s & 1+2 s & 0 \\
0 & -1-2 s & 1+2 s
\end{array}\right) .
\end{array}
$$

Recall the definition $\widetilde{\gamma}_{2}:=\frac{1}{2}\left(\gamma_{1}+\gamma_{2}\right)$ in (5.21), and recall

$$
M l(f)_{\Phi_{2}}=\mathbb{Z} \gamma_{1} \oplus \mathbb{Z} \widetilde{\gamma}_{2} \stackrel{2: 1}{\supset} \mathbb{Z} \gamma_{1} \oplus \mathbb{Z} \gamma_{2}=\left(B_{1} \oplus B_{2}\right)_{\Phi_{2}} .
$$

Thus also

$$
\begin{aligned}
M l(f)_{\Phi_{6} \Phi_{2}} & =\left\langle\delta_{1}, M_{h}\left(\delta_{1}\right), M_{h}^{2}\left(\delta_{1}\right), \delta_{2}, M_{h}\left(\delta_{2}\right), \widetilde{\gamma}_{2}\right\rangle \\
& \stackrel{2: 1}{\supset}\left(B_{1} \oplus B_{2}\right)_{\Phi_{6} \Phi_{2}},
\end{aligned}
$$

where

$\widetilde{\gamma}_{2}=\frac{1}{2}\left(\gamma_{1}+\gamma_{2}\right)=\frac{1}{2}\left(\delta_{1}-M_{h}\left(\delta_{1}\right)+M_{h}^{2}\left(\delta_{1}\right)+\delta_{2}-M_{h}\left(\delta_{2}\right)+M_{h}^{2}\left(\delta_{2}\right)\right)$.

The matrix of $L$ for the $\mathbb{Z}$-basis $\delta_{1}, M_{h}\left(\delta_{1}\right), M_{h}^{2}\left(\delta_{1}\right), \delta_{2}, M_{h}\left(\delta_{2}\right), \widetilde{\gamma}_{2}$ of $M l(f)_{\Phi_{6} \Phi_{2}}$ is

$$
\left(\begin{array}{cccccc}
2 & 2 & 2 & 0 & 0 & 1 \\
-2 & 2 & 2 & 0 & 0 & -1 \\
-2 & -2 & 2 & 0 & 0 & 1 \\
0 & 0 & 0 & 1+2 s & 0 & 1+2 s \\
0 & 0 & 0 & -1-2 s & 1+2 s & -1-2 s \\
1 & -1 & 1 & 1+2 s & -1-2 s & 3+3 s
\end{array}\right)
$$

The associated quadratic form $\left(x_{1} \ldots x_{6}\right)$ (matrix) $\left(\begin{array}{c}x_{1} \\ \vdots \\ x_{6}\end{array}\right)$ is

$$
\begin{aligned}
& \frac{1}{2}\left[\left(2 x_{1}+x_{6}\right)^{2}+\left(2 x_{2}-x_{6}\right)^{2}+\left(2 x_{3}+x_{6}\right)^{2}\right] \\
+ & \frac{1}{2}(1+2 s)\left[\left(x_{4}-x_{5}+x_{6}\right)^{2}+\left(x_{4}+x_{6}\right)^{2}+\left(x_{5}-x_{6}\right)^{2}\right] .
\end{aligned}
$$

One finds

$$
\left\{a \in M l(f)_{\Phi_{6} \Phi_{2}} \mid L(a, a)=2\right\}=\left\{ \pm M_{h}^{j}\left(\delta_{1}\right) \mid j=0,1,2\right\},
$$

and also

$$
\left\{a \in\left(B_{1} \oplus B_{2}\right)_{\Phi_{6} \Phi_{2}} \mid L(a, a)=2\right\}=\left\{ \pm M_{h}^{j}\left(\delta_{1}\right) \mid j=0,1,2\right\} .
$$


Thus any $g \in G_{\mathbb{Z}} \cup \operatorname{Aut}\left(B_{1} \oplus B_{2}, L\right)$ maps $\delta_{1}$ to an element of $\left\{ \pm M_{h}^{j}\left(\delta_{1}\right) \mid j=0,1,2\right\}$. These are cyclic generators of the Orlik block $\left(B_{1}\right)_{\Phi_{6} \Phi_{2}}$. Thus any $g \in G_{\mathbb{Z}} \cup \operatorname{Aut}\left(B_{1} \oplus B_{2}, L\right)$ maps $\left(B_{1}\right)_{\Phi_{6} \Phi_{2}}$ to itself. As $\left(B_{2}\right)_{\Phi_{6} \Phi_{2}}$ is the $L$-orthogonal sublattice within $M l(f)_{\Phi_{6} \Phi_{2}}$, such a $g$ maps also $\left(B_{2}\right)_{\Phi_{6} \Phi_{2}}$ to itself. This shows (ii) above. Especially such a $g$ maps $\left(B_{1}\right)_{\Phi_{2}}$ to itself and its generator $\gamma_{4}=\gamma_{1}$ to $\pm \gamma_{4}$. This shows (i) above.

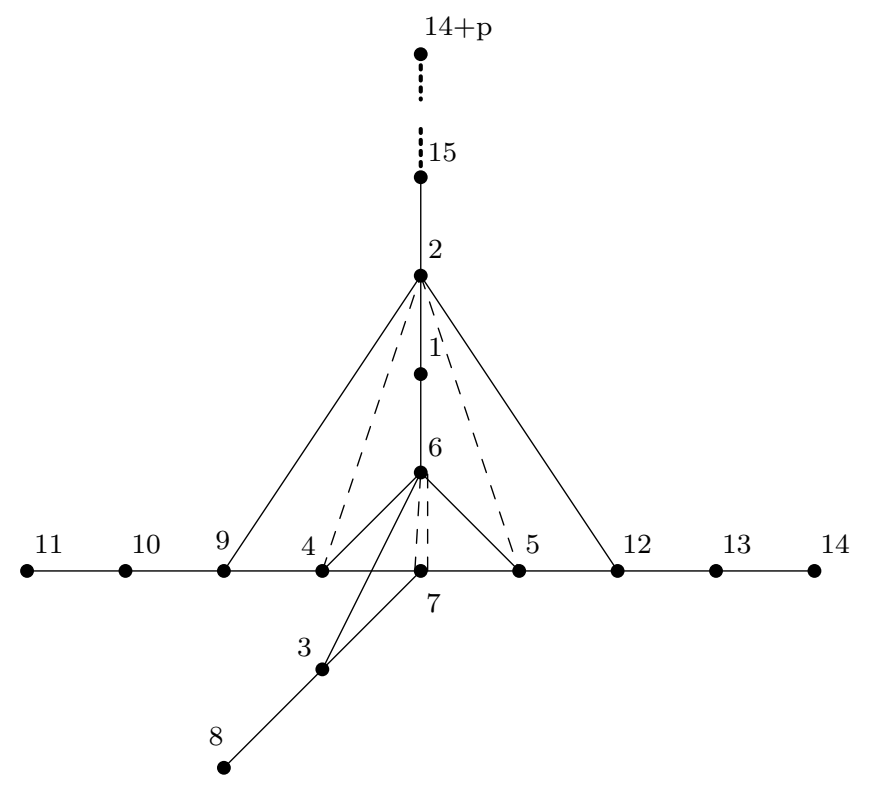

Figure 8. The CDD of a distinguished basis $e_{1}, \ldots, e_{\mu}$ for $S_{1, p}$ from [Eb81, Tabelle 6 \& Abb. 16] 
5.8. The series $S_{1, p}$. Here the monodromy acts on the distinguished basis $e_{1}, \ldots, e_{\mu}$ with the CDD in figure 8 as follows:

$$
\begin{aligned}
& e_{1} \mapsto-e_{1}-e_{2}+e_{3}+e_{4}+e_{5}+e_{6}, \\
& e_{2} \mapsto 2 e_{1}+2 e_{2}+e_{9}+e_{12}+e_{15} \text {, } \\
& e_{3} \mapsto-e_{1}-e_{6}+e_{7}+e_{8} \text {, } \\
& e_{4} \mapsto e_{2}-e_{6}+e_{7}+e_{9} \text {, } \\
& e_{5} \mapsto e_{2}-e_{6}+e_{7}+e_{12} \text {, } \\
& e_{6} \mapsto e_{1}-2 e_{2}+e_{3}+e_{4}+e_{5}+3 e_{6}-2 e_{7} \text {, } \\
& e_{7} \mapsto-2 e_{2}+e_{3}+e_{4}+e_{5}+2 e_{6}-e_{7}, \\
& e_{8} \mapsto-e_{3}-e_{8} \text {, } \\
& e_{9} \mapsto e_{10}, \\
& e_{10} \mapsto e_{11}, \\
& e_{11} \mapsto-e_{4}-e_{9}-e_{10}-e_{11}, \\
& e_{12} \mapsto e_{13}, \\
& e_{13} \mapsto e_{14}, \\
& e_{14} \mapsto-e_{5}-e_{12}-e_{13}-e_{14}, \\
& e_{14+i} \mapsto e_{15+i} \quad \text { for } 1 \leq i \leq p-1 \text {, } \\
& e_{14+p} \mapsto-e_{1}-e_{2}-e_{15}-e_{16}-\ldots-e_{14+p} .
\end{aligned}
$$

By table (5.13) the generators of the Orlik blocks $B_{1}$ and $B_{2}$ are $\beta_{1}:=$ $-e_{8}+e_{13}$ and $\beta_{2}:=e_{15}$. The monodromy acts on them as follows:

$$
\begin{aligned}
-e_{8}+e_{13} & \mapsto e_{3}+e_{8}+e_{14} \\
& \mapsto-e_{1}-e_{3}-e_{5}-e_{6}+e_{7}-e_{12}-e_{13}-e_{14} \\
& \mapsto e_{1}-e_{3}-e_{4}-e_{7}-e_{8} \\
& \mapsto e_{3}+e_{6}-e_{7}-e_{9} \\
& \mapsto e_{8}-e_{10} \\
& \mapsto-e_{3}-e_{8}-e_{11} \\
& \mapsto e_{1}+e_{3}+e_{4}+e_{6}-e_{7}+e_{9}+e_{10}+e_{11} \\
& \mapsto-e_{1}+e_{3}+e_{5}+e_{7}+e_{8} \\
& \mapsto-e_{3}-e_{6}+e_{7}+e_{12} \\
& \mapsto-e_{8}+e_{13}
\end{aligned}
$$




$$
\begin{aligned}
e_{15} & \mapsto e_{16} \mapsto \ldots \mapsto e_{14+p} \mapsto-e_{1}-e_{2}-\sum_{i=15}^{14+p} e_{i} \\
& \mapsto-e_{3}-e_{4}-e_{5}-e_{6}-e_{9}-e_{12} \\
& \mapsto-e_{3}-e_{4}-e_{5}-e_{7}-e_{8}-e_{9}-e_{10}-e_{12}-e_{13} \\
& \mapsto e_{1}-e_{4}-e_{5}+e_{6}-2 e_{7}-\sum_{j \in\{9,10,11,12,13,14\}} e_{j} \\
& \mapsto-e_{2}+e_{4}+e_{5}+2 e_{6}-2 e_{7} \mapsto-e_{15} .
\end{aligned}
$$

Thus the characteristic polynomial of $M_{h}$ on $B_{j}$ is $b_{j}$. Here the blocks $B_{1}$ and $B_{2}$ are generated by the first $\operatorname{deg} b_{1}$ respectively $\operatorname{deg} b_{2}$ of the elements above. Here $B_{1}+B_{2}=B_{1} \oplus B_{2}$ and $\left[M l(f): B_{1} \oplus B_{2}\right]=2=$ $r_{I}$ follow by the calculation of the determinant which expresses these generators of $B_{1}$ and $B_{2}$ in the distinguished basis $e_{1}, \ldots, e_{\mu}$. Then it also follows that $B_{1}$ and $B_{2}$ are primitive sublattices.

The proof of (5.5) for $S_{1,10}$ was postponed to this section and has to be given here. From now on only $S_{1,10}$ is considered. (5.25) shows that $\left(M l(f)_{\Phi_{2}}, L\right)$ is an $A_{2}$-lattice with roots $\left\{ \pm \gamma_{1}, \pm \widetilde{\gamma}_{2}, \pm\left(\widetilde{\gamma}_{2}-\gamma_{1}\right)\right\}$. Here $\gamma_{1}$ generates $\left(B_{1}\right)_{\Phi_{2}}$. We will show that $\left(B_{1}\right)_{\Phi_{10}}$ and $\pm \gamma_{1}$ satisfy the following special relationship:

$$
\begin{aligned}
& {\left[\left(\left(B_{1}\right)_{\Phi_{10}}+\mathbb{Z} \cdot a\right)_{\mathbb{Q}} \cap \operatorname{Ml}(f):\left(\left(B_{1}\right)_{\Phi_{10}}+\mathbb{Z} \cdot a\right)\right] } \\
= & \begin{cases}5 & \text { if } a= \pm \gamma_{1} \\
1 & \text { if } a \in\left\{ \pm \widetilde{\gamma}_{2}, \pm\left(\widetilde{\gamma}_{2}-\gamma_{1}\right)\right\} .\end{cases}
\end{aligned}
$$

If $a= \pm \gamma_{1}$, then

$$
\begin{aligned}
\left(\left(B_{1}\right)_{\Phi_{10}}+\mathbb{Z} \cdot a\right)_{\mathbb{Q}} \cap M l(f) & =\left(B_{1}\right)_{\Phi_{10} \Phi_{2}}=\bigoplus_{j=0}^{4} \mathbb{Z} \cdot\left(t^{j} \Phi_{5}\right)\left(M_{h}\right)\left(\beta_{1}\right), \\
\left(B_{1}\right)_{\Phi_{10}}+\mathbb{Z} \cdot a & =\left(B_{1}\right)_{\Phi_{10}}+\left(B_{1}\right)_{\Phi_{2}} \\
=\bigoplus_{j=0}^{3} \mathbb{Z} \cdot\left(t^{j} \Phi_{2} \Phi_{5}\right)\left(M_{h}\right)\left(\beta_{1}\right) & \oplus \mathbb{Z} \cdot\left(\Phi_{10} \Phi_{5}\right)\left(M_{h}\right)\left(\beta_{1}\right),
\end{aligned}
$$

so the index is

$$
\left[\bigoplus_{j=0}^{4} \mathbb{Z} \cdot t^{j}: \bigoplus_{j=0}^{3} \mathbb{Z} \cdot t^{j} \Phi_{2} \oplus \mathbb{Z} \cdot \Phi_{10}\right]=5 .
$$

Now recall that $\left(B_{1}\right)_{\Phi_{10}}$ is a primitive sublattice of $M l(f)$ and that

$$
B_{1} \subset \bigoplus_{j=1}^{14} \mathbb{Z} \cdot e_{j}, \quad \text { so }\left(B_{1}\right)_{\Phi_{10}} \subset \bigoplus_{j=1}^{14} \mathbb{Z} \cdot e_{j}
$$


Observe that

$$
\widetilde{\gamma}_{2} \equiv \widetilde{\gamma}_{2}-\gamma_{1} \equiv-\sum_{j=15}^{24} e_{j} \bmod \sum_{j=1}^{14} \mathbb{Z} \cdot e_{j} .
$$

Because of the sum $-\sum_{j=15}^{24} e_{j}$ in $\widetilde{\gamma}_{2}$ and in $\widetilde{\gamma}_{2}-\gamma_{1}$, the sublattices $\left(B_{1}\right)_{\Phi_{10}} \oplus \mathbb{Z} \cdot \widetilde{\gamma}_{2}$ and $\left(B_{1}\right)_{\Phi_{10}} \oplus \mathbb{Z} \cdot\left(\widetilde{\gamma}_{2}-\gamma_{1}\right)$ are primitive in $M l(f)$, so the index above is 1 . This shows (5.163).

Now (5.5) is an easy consequence: Consider an element $g \in G_{\mathbb{Z}}$ with $g\left(\left(\bar{B}_{1}\right)_{\Phi_{10}}\right)=\left(B_{1}\right)_{\Phi_{10}}$. It must map $\gamma_{1}$ to some root of the $A_{2^{-}}$ lattice $\left(M l(f)_{\Phi_{10}}, L\right)$. Because of $(5.163)$, the image must be $\pm \gamma_{1}$, so $g\left(\left(B_{1}\right)_{\Phi_{2}}\right)=\left(B_{1}\right)_{\Phi_{2}}$. Therefore $g\left(\left(B_{1}\right)_{\Phi_{10} \Phi_{2}}\right)=\left(B_{1}\right)_{\Phi_{10} \Phi_{2}}$ and by its $L$-orthogonality also $g\left(\left(B_{2}\right)_{\Phi_{10} \Phi_{2}}\right)=\left(B_{2}\right)_{\Phi_{10} \Phi_{2}}$.

For $S_{1,10} b_{1}=\Phi_{10} \Phi_{5} \Phi_{2}$ and $b_{2}=\Phi_{30} \Phi_{10} \Phi_{6} \Phi_{2}$, so the eigenspaces with eigenvalues different from the roots of $\Phi_{10} \Phi_{2}$ are one-dimensional and are either in $\left(B_{1}\right)_{\mathbb{C}}$ or in $\left(B_{2}\right)_{\mathbb{C}}$. This implies $(5.5)$ for $S_{1,10}$.

This finishes the proof of theorem 5.1.

\section{The group $G_{\mathbb{Z}}$ FOR The QuAdrangle Singularities}

The normal forms from [AGV85, §13] for the six families of quadrangle singularities will be listed below in section 10 . The quadrangle singularities can be seen as special 0-th members of the eight bimodal series, with the two series $W_{1, p}^{\sharp}$ and $W_{1, p}$ for $W_{1,0}$ and the two series $S_{1, p}^{\sharp}$ and $S_{1, p}$ for $S_{1,0}$.

The following table specializes the table (5.1) to the case $p=0$. For $W_{1,0}$ and $S_{1,0}$, we have chosen the specialization of the cases $W_{1, p}^{\sharp}$ and $S_{1, p}^{\sharp}$, not $W_{1, p}$ and $S_{1, p}$. The reason is that the Orlik blocks in theorem 5.1 for $W_{1, p}^{\sharp}$ and $S_{1, p}^{\sharp}$ work also for $W_{1,0}$ and $S_{1,0}$, but those for $W_{1, p}$ and $S_{1, p}$ work not for $W_{1,0}$ and $S_{1,0}$. Again $b_{1} b_{2}$ respectively $b_{1} b_{2} b_{3}$ for $Z_{1,0}$ are the characteristic polynomials of the surface singularities.

\begin{tabular}{lllllll} 
family & $\mu$ & $b_{1}$ & $b_{2}$ & $b_{3}$ & $m$ & $r_{I}$ \\
\hline$W_{1,0}$ & 15 & $\Phi_{12}$ & $\Phi_{12} \Phi_{6} \Phi_{4} \Phi_{3} \Phi_{2}$ & - & 12 & 1 \\
$S_{1,0}$ & 14 & $\Phi_{10} \Phi_{2}$ & $\Phi_{10} \Phi_{5} \Phi_{2}$ & - & 10 & 1 \\
$U_{1,0}$ & 14 & $\Phi_{9}$ & $\Phi_{9} \Phi_{3}$ & - & 9 & 1 \\
$E_{3,0}$ & 16 & $\Phi_{18} \Phi_{2}$ & $\Phi_{18} \Phi_{6} \Phi_{2}$ & - & 18 & 2 \\
$Z_{1,0}$ & 15 & $\Phi_{14} \Phi_{2}$ & $\Phi_{14} \Phi_{2}$ & $\Phi_{2}$ & 14 & 2 \\
$Q_{2,0}$ & 14 & $\Phi_{12} \Phi_{4} \Phi_{3}$ & $\Phi_{12} \Phi_{4}$ & - & 12 & 2
\end{tabular}

The following theorem on the group $G_{\mathbb{Z}}$ has a strong similarity with the analogous theorem 5.1 for the eight bimodal series. And luckily, also large parts of the proof of theorem 5.1 apply also to the case $p=0$. 
We do not have (5.4) $G_{\mathbb{Z}}=\operatorname{Aut}\left(\bigoplus_{j \geq 1} B_{j}, L\right)$ for $E_{3,0}, Z_{1,0}, Q_{2,0}$. But we have an analogue of the substitute (5.5) for $S_{1,10}$, the formula (6.4). Contrary to theorem 5.1, we need and give a precise description of the induced Fuchsian group. The proof uses theorem 3.6. A part of the proof (a surjectivity) is postponed to section 10. For each family, denote $\zeta:=e^{2 \pi i / m} \in S^{1} \subset \mathbb{C}$.

Theorem 6.1. For any surface singularity $f$ in any of the six families of quadrangle singularities, the following holds.

(a) (See definition 2.3 for the notion Orlik block) For all families except $Z_{1,0}$, there are Orlik blocks $B_{1}, B_{2} \subset M l(f)$, and for $Z_{1,0}$, there are Orlik blocks $B_{1}, B_{2}, B_{3} \subset M l(f)$ with the following properties. The characteristic polynomial $p_{B_{j}}$ of the monodromy on $B_{j}$ is $b_{j}$. The sum $\sum_{j \geq 1} B_{j}$ is a direct sum $\bigoplus_{j \geq 1} B_{j}$, and it is a sublattice of $M l(f)$ of full rank $\mu$ and of index $r_{I}$. Define

$$
\widetilde{B}_{1}:= \begin{cases}B_{1} & \text { for all series except } Z_{1, p}, \\ B_{1} \oplus B_{3} & \text { for the series } Z_{1, p} .\end{cases}
$$

Then

$$
\begin{aligned}
L\left(\widetilde{B}_{1}, B_{2}\right) & =0=L\left(B_{2}, \widetilde{B}_{1}\right) \\
g \in G_{\mathbb{Z}} \text { with } g\left(\left(B_{1}\right)_{\Phi_{10}}\right)=\left(B_{1}\right)_{\Phi_{10}} & \Rightarrow g\left(B_{j}\right)=B_{j} \text { for } j \geq 1 .(6.4
\end{aligned}
$$

(b) The eigenspace $M l(f)_{\zeta} \subset M l(f)_{\mathbb{C}}$ is 2-dimensional. The hermitian form $h_{\zeta}$ on it from lemma 2.2 (a) with $h_{\zeta}(a, b):=\sqrt{-\zeta} \cdot L(a, \bar{b})$ for $a, b \in M l(f)_{\zeta}$ is nondegenerate and indefinite, so $\mathbb{P}\left(M l(f)_{\zeta}\right) \cong \mathbb{P}^{1}$ contains a half-plane

$$
\mathcal{H}_{\zeta}:=\left\{\mathbb{C} \cdot a \mid a \in M l(f)_{\zeta} \text { with } h_{\zeta}(a, a)<0\right\} \subset \mathbb{P}\left(M l(f)_{\zeta}\right) .
$$

Therefore the group $\operatorname{Aut}\left(M l(f)_{\zeta}, h_{\zeta}\right) / S^{1}$. id is isomorphic to $\operatorname{PSL}(2, \mathbb{R})$. The homomorphism

$$
\Psi: G_{\mathbb{Z}} \rightarrow \operatorname{Aut}\left(M l(f)_{\zeta}, h_{\zeta}\right) / S^{1} \cdot \mathrm{id},\left.\quad g \mapsto g\right|_{M l(f)_{\zeta}} \bmod S^{1} \cdot \mathrm{id},
$$

is well-defined. $\Psi\left(G_{\mathbb{Z}}\right)$ is an infinite Fuchsian group acting on the halfplane $\mathcal{H}_{\zeta}$. It is a triangle group of the same type as in theorem 3.6, so of the following type:

$$
\begin{array}{l|l|l|l|l}
W_{1,0} & S_{1,0} & E_{3,0} \& U_{1,0} & Z_{1,0} & Q_{2,0} \\
(2,12,12) & (2,10,10) & (2,3,18) & (2,3,14) & (2,3,12)
\end{array}
$$

And

$$
\operatorname{ker} \Psi=\left\{ \pm M_{h}^{k} \mid k \in \mathbb{Z}\right\}
$$


Proof: (a) We choose again (as in section 5) for each of the six cases a distinguished basis with the Coxeter-Dynkin diagram in [Eb81, Tabelle 6 and Abb. 16].

The diagrams for $W_{1, p}^{\sharp}$ and $W_{1, p}$ specialize both to the same diagram for $W_{1,0}$. Though the description of the action of the monodromy on the distinguished basis for $W_{1, p}^{\sharp}$ in 5.1 specializes to $W_{1,0}$, but not the description for $W_{1, p}$ in 5.7. In the latter case $e_{2} \mapsto 2 e_{1}+2 e_{2}+e_{8}+$ $e_{12}+e_{16}$, but $e_{16}$ does not exist for $W_{1,0}$. Therefore we work with the specialization to $p=0$ of the formulas for $W_{1, p}^{\sharp}$ in subsection 5.1 .

The same applies to $S_{1,0}$. There we work with the specialization to $p=0$ of the formulas for $S_{1, p}^{\sharp}$ in subsection 5.2 .

The Orlik blocks $B_{1}$ and $B_{2}$ (and $B_{3}$ for $Z_{1,0}$ ) are defined as in the proof of theorem 5.1, there for $p>0$, now for $p=0$. By the same arguments, the sum $\sum_{j \geq 1} B_{j}$ is a direct sum $\bigoplus_{j \geq 1} B_{j}$ and a sublattice of $M l(f)$ of full rank $\mu$ and index $r_{I}$, and (6.3) holds.

With respect to part (a), it rests to show (6.4). In the cases $W_{1,0}$ and $U_{1,0}$, it is trivial as $r_{I}=1$ and $b_{1}=\Phi_{m}$ and $B_{1}$ and $B_{2}$ are $L$-orthogonal.

In the cases $S_{1,0}, E_{3,0}, Z_{1,0}$ and $Q_{2,0}$, the proof will be similar to the proof of (5.5) for $S_{1,10}$ in subsection 5.8. First we treat $S_{1,0}, E_{3,0}$ and $Z_{1,0}$ together, then we come to $Q_{2,0}$.

The following formulas in the proof of part (a) of theorem 5.1 specialize to the cases $S_{1,0}, E_{3,0}$ and $Z_{1,0}$ : (5.10)-(5.26), 5.28, (5.33), (5.35).

The quadratic forms in $(5.26)$ give now the following variants of (5.27) and (5.29):

$$
\begin{aligned}
\left\{a \in M l(f)_{\Phi_{2}} \mid L(a, a)=5\right\}= & \left\{ \pm \gamma_{1}, \pm \gamma_{2}\right\} \quad \text { for } S_{1,0}, \\
\left\{a \in M l(f)_{\Phi_{2}} \mid L(a, a)=6\right\}= & \left\{ \pm \gamma_{1}, \pm \widetilde{\gamma}_{2}, \pm\left(\widetilde{\gamma}_{2}-\gamma_{1}\right)\right\} \quad \text { for } E_{3,0}, \\
\left\{a \in M l(f)_{\Phi_{2}} \mid L(a, a)=5\right\}= & \left\{ \pm\left(\gamma_{1}-3 \gamma_{2}\right),\right. \\
& \left. \pm \widetilde{\gamma}_{2}, \pm\left(\widetilde{\gamma}_{2}-\gamma_{2}\right)\right\} \quad \text { for } Z_{1,0} .
\end{aligned}
$$

The first element (up to sign) of each of these three sets generates in the corresponding case $\left(B_{1}\right)_{\Phi_{2}}$. We claim that $\left(B_{1}\right)_{\Phi_{m}}$ and this first element satisfy the following special relationship. For $a$ in any of these three sets define

\begin{tabular}{|c|c|c|c|c|}
\hline & $S_{1,0}$ & $E_{3,0}$ & & $Z_{1,0}$ \\
\hline$r(a)$ & $\pm \gamma_{1} \quad 5$ & $\pm \gamma_{1}$ & 3 & $\pm\left(\gamma_{1}-2 \gamma_{3}\right)$ \\
\hline$r(a)$ & $\pm \gamma_{2}$ & $\pm \widetilde{\gamma}_{2}, \pm\left(\widetilde{\gamma}_{2}-\gamma_{1}\right)$ & 1 & $\pm \tilde{\gamma}_{2}, \pm\left(\widetilde{\gamma}_{2}-\gamma_{2}\right)$ \\
\hline
\end{tabular}

$$
r(a):=\left[\left(\left(B_{1}\right)_{\Phi_{m}}+\mathbb{Z} \cdot a\right)_{\mathbb{Q}} \cap M l(f):\left(\left(B_{1}\right)_{\Phi_{m}}+\mathbb{Z} \cdot a\right)\right] \in \mathbb{Z}_{\geq 1} \cdot(6
$$

Then we claim: 
The proof is the same as the proof of 5.163 for $S_{1,10}$ in subsection 5.8. We use that for any unitary polynomial $p(t) \in \mathbb{Z}[t]$

$$
\left[\bigoplus_{j=0}^{\operatorname{deg} p} \mathbb{Z} \cdot t^{j}: \bigoplus_{j=0}^{\operatorname{deg} p-1} \mathbb{Z} \cdot t^{j} \Phi_{2} \oplus \mathbb{Z} \cdot p(t)\right]=|p(-1)|
$$

and

$$
\Phi_{10}(-1)=5, \quad \Phi_{18}(-1)=3, \quad \Phi_{14}(-1)=7 .
$$

We also use

$$
B_{1} \subset \sum_{j=1}^{m_{1}} \mathbb{Z} \cdot e_{j} \quad \text { with } m_{1}:=8,9,10 \text { for } S_{1,0}, E_{3,0}, Z_{1,0}
$$

and that the elements in the second line of 6.11 are modulo $\sum_{j=1}^{m_{1}} \mathbb{Z} \cdot e_{j}$

$$
\begin{array}{ll}
S_{1,0}: & \gamma_{2} \equiv e_{9}+e_{11}+e_{12}+e_{14}, \\
E_{3,0}: & \widetilde{\gamma}_{2} \equiv e_{10}+e_{12}+e_{14}+e_{16}, \quad \widetilde{\gamma}_{2}-\gamma_{1} \equiv \widetilde{\gamma}_{2}, \\
Z_{1,0}: & \widetilde{\gamma}_{2} \equiv e_{11}+e_{13}+e_{15}, \quad \widetilde{\gamma}_{2}-\gamma_{2} \equiv-\widetilde{\gamma}_{2} .
\end{array}
$$

Therefore $\left(B_{1}\right)_{\Phi_{m}}+\mathbb{Z} \cdot a$ for these elements $a$ is primitive in $M l(f)$, and thus $r(a)=1$.

The derivation of (6.4) from (6.11) and $(6.9)$ for $S_{1,0}, E_{3,0}$ and $Z_{1,0}$ is almost the same as the derivation of (5.5) from $(5.163)$ for $S_{1,10}$ in subsection 5.8 .

The only additional argument concerns $B_{3}=\mathbb{Z} \cdot \gamma_{3}$ in the case $Z_{1,0}$. Because of (5.28) any $g \in G_{\mathbb{Z}}$ maps $B_{3}$ to itself. Because of $L\left(\gamma_{1}-\right.$ $\left.2 \gamma_{3}, \gamma_{3}\right)=1 \neq 0, B_{3}$ and $\left(B_{1}\right)_{\Phi_{2}}$ are glued together: If $g=\varepsilon \cdot \mathrm{id}$ on $\left(B_{1}\right)_{\Phi_{2}}$ for some $\varepsilon \in\{ \pm 1\}$, then $g=\varepsilon \cdot$ id on $B_{3}$.

Now we come to $Q_{2,0}$. The formulas (5.113)-(5.116), (5.118)-(5.119), (5.122)-(5.133), 5.136-5.139 are also valid for $p=0$ respectively $s=0$. The quadratic form in (5.133) now gives the following variant of (5.134):

$$
\begin{array}{r}
A:=\left\{\gamma_{1}, \gamma_{3}, \gamma_{1}-\gamma_{3}+M_{h}\left(\gamma_{3}\right), \gamma_{1}-M_{h}\left(\gamma_{1}\right)+M_{h}\left(\gamma_{3}\right)\right\} \\
\left\{b \in M l(f)_{\Phi_{4}} \mid L(b, b)=3\right\}=\bigcup_{a \in A}\left\{ \pm a, \pm M_{h}(a)\right\},
\end{array}
$$

so these are 16 elements which come in 4 sets of 4 elements such that each set is $M_{h}$-invariant. Recall that $M_{h}^{2}=-\mathrm{id}$ on $M l(f)_{\Phi_{4}}$. The set $\left\{ \pm \gamma_{1}, \pm M_{h}\left(\gamma_{1}\right)\right\}$ generates $\left(B_{1}\right)_{\Phi_{4}}$. 
We claim that $\left(B_{1}\right)_{\Phi_{12}}$ and this set satisfy the following special relationship. For $a \in A$ define the index

$$
\begin{aligned}
r(a):= & {\left[\left(\left(B_{1}\right)_{\Phi_{12}}+\mathbb{Z} \cdot a+\mathbb{Z} \cdot M_{h}(a)\right)_{\mathbb{Q}} \cap M l(f)\right.} \\
& \left.:\left(\left(B_{1}\right)_{\Phi_{12}}+\mathbb{Z} \cdot a+\mathbb{Z} \cdot M_{h}(a)\right)\right] \in \mathbb{Z}_{\geq 1} .
\end{aligned}
$$

Then we claim:

$$
r(a)= \begin{cases}9 & \text { for } a=\gamma_{1}, \\ 1 & \text { for } a \in\left\{\gamma_{3}, \gamma_{1}-M_{h}\left(\gamma_{1}\right)+M_{h}\left(\gamma_{3}\right)\right\}, \\ 1 \text { or } 2 & \text { for } a=\gamma_{1}-\gamma_{3}+M_{h}\left(\gamma_{3}\right) .\end{cases}
$$

$r\left(\gamma_{1}\right)=9$ holds because of

$$
\begin{aligned}
& \left(\left(B_{1}\right)_{\Phi_{12}}+\mathbb{Z} \cdot \gamma_{1}+\mathbb{Z} \cdot M_{h}\left(\gamma_{1}\right)\right)_{\mathbb{Q}} \cap M l(f) \\
= & \left(B_{1}\right)_{\Phi_{12} \Phi_{4}}=\bigoplus_{j=0}^{5} \mathbb{Z} \cdot\left(t^{j} \Phi_{3}\right)\left(M_{h}\right)\left(\beta_{1}\right), \\
& \left(B_{1}\right)_{\Phi_{12}}+\mathbb{Z} \cdot \gamma_{1}+\mathbb{Z} \cdot M_{h}\left(\gamma_{1}\right) \\
= & \bigoplus_{j=0}^{3} \mathbb{Z} \cdot\left(t^{j} \Phi_{4} \Phi_{3}\right)\left(M_{h}\right)\left(\beta_{1}\right) \oplus \bigoplus_{j=0}^{1} \mathbb{Z} \cdot\left(t^{j} \Phi_{12} \Phi_{3}\right)\left(M_{h}\right)\left(\beta_{1}\right),
\end{aligned}
$$

and thus

$$
r\left(\gamma_{1}\right)=\left[\bigoplus_{j=0}^{5} \mathbb{Z} \cdot t^{j}: \bigoplus_{j=0}^{3} \mathbb{Z} \cdot t^{j} \Phi_{4} \oplus \bigoplus_{j=0}^{1} \mathbb{Z} \cdot t^{j} \Phi_{12}\right]=3 \cdot 3 .
$$

For $a \in A-\left\{\gamma_{1}\right\}, r(a) \in\{1,2\}$ holds because of

$$
B_{1} \subset \sum_{j=1}^{10} \mathbb{Z} \cdot e_{j}
$$

and because the elements $a$ and $M_{h}(a)$ for $a \in A-\left\{\gamma_{1}\right\}$ are modulo $\sum_{j=1}^{10} \mathbb{Z} \cdot e_{j}$

$$
\begin{aligned}
\gamma_{1} & \equiv-e_{13}-e_{14}, \\
M_{h}\left(\gamma_{1}\right) & \equiv e_{12}+e_{13}, \\
\gamma_{1}-\gamma_{3}+M_{h}\left(\gamma_{3}\right) & \equiv e_{12}+2 e_{13}+e_{14}, \\
M_{h}\left(\gamma_{1}-\gamma_{3}+M_{h}\left(\gamma_{3}\right)\right) & \equiv-e_{12}+e_{14}, \\
\gamma_{1}-M_{h}\left(\gamma_{1}\right)+M_{h}\left(\gamma_{3}\right) & \equiv e_{12}+e_{13}, \\
M_{h}\left(\gamma_{1}-M_{h}\left(\gamma_{1}\right)+M_{h}\left(\gamma_{3}\right)\right) & \equiv e_{13}+e_{14} .
\end{aligned}
$$

The derivation of (6.4) for $Q_{2,0}$ from 6.17 and 6.19 is a simple variant of the derivation of (5.5) from (5.163) for $S_{1,10}$ in subsection 5.8. Consider an element $g \in G_{\mathbb{Z}}$ with $g\left(\left(B_{1}\right)_{\Phi_{12}}\right)=\left(B_{1}\right)_{\Phi_{12}}$. Because 
of (6.17), it maps the set $\left\{ \pm \gamma_{1}, \pm M_{h}\left(\gamma_{1}\right)\right\}$ to one of the four sets on the right hand side of (6.17). Because of (6.19), the image must be the set $\left\{ \pm \gamma_{1}, \pm M_{h}\left(\gamma_{1}\right)\right\}$ itself. As this set generates $\left(B_{1}\right)_{\Phi_{4}}, g$ maps $\left(B_{1}\right)_{\Phi_{4}}$ to itself. Then $g$ maps the sets $\left(B_{1}\right)_{\Phi_{12} \Phi_{4}}, B_{1}=\left(B_{1}\right)_{\Phi_{12} \Phi_{4} \Phi_{3}}$ and $B_{2}=\left(B_{2}\right)_{\Phi_{12} \Phi_{4}}$ to themselves. This finishes the proof of part (a).

(b) All the formulas and arguments in the proof of part (c) of theorem 5.1 for the cases $W_{1,12 r}^{\sharp}, S_{1,10 r}^{\sharp}, U_{1,9 r}, E_{3,18 r}, Z_{1,14 r}$ and $Q_{2,12 r}$ are also valid for $r=0$.

In step 3 now (6.4) is used instead of (5.4), just as $(5.5)$ for $S_{1,10}$. Therefore (6.7) holds and $\Psi\left(G_{\mathbb{Z}}\right)$ is an infinite Fuchsian group.

By table (5.72), the remarks 3.5 and theorem 3.6, $\Psi\left(G_{\mathbb{Z}}\right)$ is a subgroup of a triangle group of the same type as in theorem 3.6 , for each case. The proof of theorem 10.1 will show that it is the full triangle group.

\section{Gauss-Manin connection and Brieskorn lattice}

The Gauss-Manin connection of isolated hypersurface singularities had been considered first by Brieskorn in 1970 [Br70]. Since then it had been described by many people in many papers (K. Saito, Greuel, Pham, Varchenko, M. Saito, Hertling, and others). The following presentation will be short on the $\mathcal{D}$-module foundations. But it will make the relations between the different pairings precise (more precise than anywhere in the literature). And it will emphasize the computational aspects. Other versions are in [AGV88, [He93], [He95], Ku98] and [He02.

Throughout most of this section, we consider a fixed isolated hypersurface singularity $f:\left(\mathbb{C}^{n+1}, 0\right) \rightarrow(\mathbb{C}, 0)$, its flat cohomology bundle $\bigcup_{\tau \in \Delta^{*}} H^{n}\left(f^{-1}(\tau), \mathbb{C}\right)$, and the space $H_{\mathbb{C}}^{\infty}$ of global flat multi-valued sections (see section 4 for $H_{\mathbb{C}}^{\infty}$ ).

First we define the elementary sections es $(A, \alpha)$, the spaces $C^{\alpha}$ which they generate, and the $V$-filtration.

Any global flat multi-valued section $A \in H_{\lambda}^{\infty}$ and any choice of $\alpha \in \mathbb{Q}$ with $e^{-2 \pi i \alpha}=\lambda$ leads to a holomorphic univalued section with specific growth condition at $0 \in \Delta$, the elementary section es $(A, \alpha)$ with

$$
e s(A, \alpha)(\tau):=e^{\log \tau\left(\alpha-\frac{N}{2 \pi i}\right)} \cdot A(\log \tau) .
$$

Recall that $N$ is the nilpotent part of the monodromy $M_{h}$. Denote by $C^{\alpha}$ the $\mathbb{C}$-vector space of all elementary sections with fixed $\alpha$ and $\lambda$. The map

$$
\psi_{\alpha}:=e s(., \alpha): H_{\lambda}^{\infty} \rightarrow C^{\alpha}
$$


is an isomorphism. The space $V^{\text {mod }}:=\bigoplus_{\alpha \in(-1,0]} \mathbb{C}\{\tau\}\left[\tau^{-1}\right] \cdot C^{\alpha}$ is the space of all germs at 0 of the sheaf of holomorphic sections on the flat cohomology bundle with moderate growth at 0 . The KashiwaraMalgrange $V$-filtration is given by the subspaces

$$
V^{\alpha}:=\bigoplus_{\beta \in[\alpha, \alpha+1)} \mathbb{C}\{\tau\} \cdot C^{\beta}, \quad V^{>\alpha}:=\bigoplus_{\beta \in(\alpha, \alpha+1]} \mathbb{C}\{\tau\} \cdot C^{\beta} .
$$

It is a decreasing filtration by free $\mathbb{C}\{\tau\}$-modules of rank $\mu$ with $\operatorname{Gr}_{V}^{\alpha}=$ $V^{\alpha} / V^{>\alpha} \cong C^{\alpha}$. And

$$
\begin{aligned}
\tau: C^{\alpha} \rightarrow C^{\alpha+1} \text { bijective, } & \tau \cdot \operatorname{es}(A, \alpha)=e s(A, \alpha+1), \\
\partial_{\tau}: C^{\alpha} \rightarrow C^{\alpha-1} \text { bijective } & \text { if } \alpha \neq 0, \\
\tau \partial_{\tau}-\alpha: C^{\alpha} \rightarrow C^{\alpha} \text { nilpotent, } & \left(\tau \partial_{\tau}-\alpha\right) \operatorname{es}(A, \alpha)=e s\left(\frac{-N}{2 \pi i} A, \alpha\right) .
\end{aligned}
$$

Therefore $\partial_{\tau}^{-1}: V^{>-1} \rightarrow V^{>0}$ is an isomorphism, and $V^{>-1}$ is a free $\mathbb{C}\left\{\left\{\partial_{\tau}^{-1}\right\}\right\}$-module of rank $\mu$.

With the polarizing form $S$ (see $(4.20)$ ), we define a $\partial_{\tau}^{-1}$-sesquilinear pairing $K_{f}$ on $V^{>-1}$. Its restriction to the Brieskorn lattice will be the restriction of K. Saito's higher residue pairings to the Brieskorn lattice (which he defined on an extension of the Brieskorn lattice to a universal unfolding).

Lemma 7.1. A unique pairing

$$
K_{f}: V^{>-1} \times V^{>-1} \rightarrow \mathbb{C}\left\{\left\{\partial_{\tau}^{-1}\right\}\right\}
$$

with the properties in (7.6)-(7.9) exists. In (7.6) and (7.7) $A \in$ $H_{e^{-2 \pi i \alpha}}^{\infty}, B \in H_{e^{-2 \pi i \beta}}^{\infty}$.

$$
\begin{aligned}
K_{f}(e s(A, \alpha), e s(B, \beta))= & \frac{1}{(2 \pi i)^{n}} S(A, B) \cdot \partial_{\tau}^{-1}, \\
& \text { for } \alpha, \beta \in(-1,0), \alpha+\beta=-1, \\
K_{f}(e s(A, \alpha), e s(B, \beta))= & \frac{-1}{(2 \pi i)^{n+1}} S(A, B) \cdot \partial_{\tau}^{-2}, \\
& \text { for } \alpha=\beta=0, \\
K_{f}: C^{\alpha} \times C^{\beta} \rightarrow 0= & \text { for } \alpha, \beta \in \mathbb{R}_{>-1}, \alpha+\beta \notin \mathbb{Z}, \\
\partial_{\tau}^{-1} \cdot K_{f}(a, b)= & K_{f}\left(\partial_{\tau}^{-1} a, b\right)=K_{f}\left(a,-\partial_{\tau}^{-1} b\right) \\
& \text { for } a, b \in V^{>-1} .
\end{aligned}
$$

It satisfies also (for $\alpha, \beta \in \mathbb{R}_{>-1}$ )

$$
\begin{aligned}
K_{f}: C^{\alpha} \times C^{\beta} \rightarrow \mathbb{C} \cdot \partial_{\tau}^{-\alpha-\beta-2} & \text { if } \alpha+\beta \in \mathbb{Z}, \\
K_{f}(\tau a, b)-K_{f}(a, \tau b)=\left[\tau, K_{f}(a, b)\right] & \text { for } a, b \in V^{>-1},
\end{aligned}
$$


where $\left[\tau, \partial_{\tau}^{-k}\right]=k \partial_{\tau}^{-k-1}$. If one writes $K_{f}(a, b)=\sum_{k \geq 1} K_{f}^{(-k)}(a, b) \cdot \partial_{\tau}^{-k}$ with $K_{f}^{(k)}(a, b) \in \mathbb{C}$, then $K_{f}^{(-k)}$ is $(-1)^{k+n+1}$-symmetric.

Proof: It is clear that (7.6)-(7.9) define a unique $\partial_{\tau}^{-1}$-sesquilinear pairing on $V^{>-1}$. Its $\partial_{\tau}^{-1}$-sesquilinearity gives (7.10). One checks (7.11) with $(7.4)$ and the infinitesimal $N$-invariance of $S$. The symmetry of the $K_{f}^{(k)}$ follows from the symmetry of $S$ and the $\partial_{\tau}^{-1}$-sesquilinearity of $K_{f}$.

Remark 7.2. In the sections 9 and 10 , we will prove the global Torelli conjecture for many families of marked bimodal surface singularities. We want to claim that it follows also for all suspensions of these families, and also for the curve singularities, if the surface singularities are themselves suspensions of curve singularities.

The Milnor lattices of $f$ and $f+x_{n+1}^{2}$ are up to a sign uniquely isomorphic. The normalized Seifert form $L^{\text {hnor }}$ and the group $G_{\mathbb{Z}}$ are the same, there is no problem.

But the Brieskorn lattices of $f$ and $f+x_{n+1}^{2}$ are not isomorphic. In He93, the second author had a lemma saying that they are sufficiently similar and vary in the same way in $\mu$-constant families.

Stronger and more elegant is the specialization to $f+x_{n+1}^{2}$ of a ThomSebastiani formula. But that requires to look at a Fourier-Laplace transformation. In the present situation of sections of moderate growth, this can be done in a nice and explicit way. Lemma 7.3, definition 7.4 and theorem 7.5 do a good part of the work. Theorem 7.9 gives a ThomSebastiani formula for a Fourier-Laplace transform of the Brieskorn lattice. Theorem 7.7 states well known properties of the Brieskorn lattice.

The pairing in lemma 7.3 had been considered first by Pham [Ph85], see remark 7.6 (i).

Lemma 7.3. Let $\gamma_{-\pi}: H^{n}\left(f^{-1}(z), \mathbb{C}\right) \rightarrow H^{n}\left(f^{-1}(-z), \mathbb{C}\right)$ (respectively $\gamma_{\pi}$ ) be the isomorphism by flat shift in mathematically negative (respectively positive) direction. Define a pairing

$$
\begin{array}{r}
P: H^{n}\left(f^{-1}(z), \mathbb{C}\right) \times H^{n}\left(f^{-1}(-z), \mathbb{C}\right) \rightarrow \mathbb{C} \text { for } z \neq 0 \\
\text { by } \quad P(a, b):=\frac{1}{(2 \pi i)^{n+1}} \cdot L^{n o r}\left(a, \gamma_{-\pi}(b)\right) .
\end{array}
$$

It is $(-1)^{n+1}$-symmetric and nondegenerate and takes values in $(2 \pi i)^{-(n+1)} \cdot \mathbb{Z}$ on $H^{n}\left(f^{-1}(z), \mathbb{Z}\right) \times H^{n}\left(f^{-1}(-z), \mathbb{Z}\right)$. It is flat, i.e. it has constant values on pairs of flat sections in the cohomology bundle. 
Proof: The only property which might not be immediately obvious, is the $(-1)^{n+1}$-symmetry. It follows from the flatness, from $M_{h} \gamma_{-\pi}=\gamma_{\pi}$ and (4.15):

$$
\begin{aligned}
(2 \pi i)^{n+1} \cdot P(b, a) & =L^{n o r}\left(b, \gamma_{-\pi} a\right)=(-1)^{n+1} L^{n o r}\left(M_{h} \gamma_{-\pi} a, b\right) \\
& =(-1)^{n+1} L^{n o r}\left(\gamma_{\pi} a, b\right)=(-1)^{n+1} L^{n o r}\left(a, \gamma_{-\pi} b\right) \\
& =(2 \pi i)^{n+1} \cdot(-1)^{n+1} \cdot P(a, b) .
\end{aligned}
$$

Definition 7.4. [He02, (7.47)] For each $\alpha \in \mathbb{R}$ define the automorphism

$$
\begin{aligned}
G^{(\alpha)} & : H_{e^{-2 \pi i \alpha}}^{\infty} \rightarrow H_{e^{-2 \pi i \alpha}}^{\infty} \\
G^{(\alpha)} & :=\sum_{k \geq 0} \frac{1}{k !} \Gamma^{(k)}(\alpha) \cdot\left(\frac{-N}{2 \pi i}\right)^{k}={ }^{\prime \prime} \Gamma\left(\alpha \cdot \mathrm{id}+\frac{-N}{2 \pi i}\right) "
\end{aligned}
$$

Define the automorphism

$$
G:=\sum_{\alpha \in(-1,0]} G^{(\alpha)}: H_{\mathbb{C}}^{\infty} \rightarrow H_{\mathbb{C}}^{\infty} .
$$

The following theorem was first formulated in He03, Proposition 7.7]. A detailed proof is in [BH17, Theorem 5.2]. The most difficult part is the proof of 7.21 .

Theorem 7.5. (a) Let $\tau$ and $z$ both be coordinates on $\mathbb{C}$. For $\alpha>0$ and $A \in H_{e^{-2 \pi i \alpha}}^{\infty}$, the Fourier-Laplace transformation $F L$ with

$$
F L(e s(A, \alpha-1)(\tau))(z):=\int_{0}^{\infty \cdot z} e^{-\tau / z} \cdot \operatorname{es}(A, \alpha-1)(\tau) d \tau
$$

is well defined and maps the elementary section es $(A, \alpha-1)(\tau)$ in $\tau$ to the elementary section

$$
F L(e s(A, \alpha-1)(\tau))(z)=e s\left(G^{(\alpha)} A, \alpha\right)(z)
$$

in $z$.

(b) It extends to a well defined isomorphism

$$
F L: \sum_{\alpha \in(-1,0]} \mathbb{C}\left\{\partial_{\tau}^{-1}\right\} \cdot C_{\tau}^{\alpha} \rightarrow V_{z}^{>0}
$$


Here the indices $\tau$ at $C^{\alpha}$ and $z$ at $V^{>0}$ indicate that the coordinate $\tau$ respectively $z$ has to be used. It satisfies for $a, b \in \sum_{\alpha \in(-1,0]} \mathbb{C}\left\{\partial_{\tau}^{-1}\right\} \cdot C_{\tau}^{\alpha}$

$$
\begin{aligned}
F L\left(\partial_{\tau}^{-1} a\right) & =z \cdot F L(a) \\
F L(\tau \cdot a) & =z^{2} \partial_{z} F L(a) \\
P(F L(a), F L(b)) & =\sum_{k \geq 1} c_{k} z^{l} \quad \text { if } K_{f}(a, b)=\sum_{k \geq 1} c_{k} \partial_{\tau}^{-k} .
\end{aligned}
$$

Remarks 7.6. (i) Pham Ph85] defined the pairing $P$ in lemma 7.3 starting with an intersection form for Lefschetz thimbles. In our situation, $H_{n}\left(f^{-1}(z), \mathbb{Z}\right)$ for $z \in \Delta^{*}$ is canonically isomorphic to the $\mathbb{Z}$ module generated by Lefschetz thimbles above the straight path from 0 to $z$. And it is easy to see that the pairing

$$
\begin{array}{r}
(-1)^{n(n+1) / 2} \cdot L^{\text {hnor }}\left(., \gamma_{-\pi}\right): \\
H_{n}\left(f^{-1}(z), \mathbb{Z}\right) \times H_{n}\left(f^{-1}(-z), \mathbb{Z}\right) \rightarrow \mathbb{Z}
\end{array}
$$

for $z \in \Delta^{*}$ is the intersection form for Lefschetz thimbles He05. This formula connects lemma 7.3 with Pham's definition.

(ii) Neither Pham nor K. Saito knew the definition 7.2 of $K_{f}$ with the polarizing form $S$. Pham had the version of (7.21) with K. Saito's higher residue pairings [SaK83] instead of $K_{f}$. He did not consider explicitly the automorphisms $G^{(\alpha)}$ and (7.17).

(iii) Because of $(7.19)$, we have to consider on the left hand side of (7.18) and in 7.19-7.21 the subspace $\sum_{\alpha \in(-1,0]} \mathbb{C}\left\{\partial_{\tau}^{-1}\right\} \cdot C_{\tau}^{\alpha}$ of $V_{\tau}^{>-1}$. The convergence condition is stronger.

Now we come to the Brieskorn lattice. It is a free $\mathbb{C}\{\tau\}$-module $H_{0}^{\prime \prime}(f) \subset V^{>-1}$ of rank $\mu$ which had first been studied by Brieskorn [Br70]. The name Brieskorn lattice is due to [SaM89], the notation $H_{0}^{\prime \prime}(f)$ is from [Br70]. The Brieskorn lattice is generated by germs of sections $s[\omega]$ from holomorphic $(n+1)$-forms $\omega \in \Omega_{X}^{n+1}$ : Integrating the Gelfand-Leray form $\left.\frac{\omega}{d f}\right|_{f^{-1}(\tau)}$ over cycles in $H_{n}\left(f^{-1}(\tau), \mathbb{C}\right)$ gives a holomorphic section $s[\omega]$ in the cohomology bundle, whose germ $s[\omega]_{0}$ at 0 is in fact in $V^{>-1}$ (this was proved first by Malgrange). The following theorem collects well known properties of the Brieskorn lattice. Afterwards we make comments on their proofs. See also [He02]. 
Theorem 7.7. Algebraic properties:

$$
\begin{aligned}
H_{0}^{\prime \prime}(f) & \cong \Omega_{\mathbb{C}^{n+1}, 0}^{n+1} / d f \wedge d \Omega_{\mathbb{C}^{n+1}, 0}^{n-1}, \\
\partial_{\tau}^{-1}: H_{0}^{\prime \prime}(f) & \cong H_{0}^{\prime}(f) \subset H_{0}^{\prime \prime}(f) \\
\text { with } H_{0}^{\prime}(f) & \cong d f \wedge \Omega_{\mathbb{C}^{n+1}, 0}^{n} / d f \wedge d \Omega_{\mathbb{C}^{n+1}, 0}^{n-1}, \\
\text { and } \partial_{\tau}: s[d f \wedge \eta]_{0} & \mapsto s[d \eta]_{0} .
\end{aligned}
$$

Compatibility with $K_{f}: K_{f}$ is the restriction to $H_{0}^{\prime \prime}(f)$ of $K$. Saito's higher residue pairings. It satisfies

$$
K_{f}: H_{0}^{\prime \prime}(f) \times H_{0}^{\prime \prime}(f) \rightarrow \partial_{\tau}^{-n-1} \cdot \mathbb{C}\left\{\left\{\partial_{\tau}^{-1}\right\}\right\} .
$$

The leading part

$$
K_{f}^{(-n-1)}: H_{0}^{\prime \prime}(f) / H_{0}^{\prime}(f) \times H_{0}^{\prime \prime}(f) / H_{0}^{\prime}(f) \rightarrow \mathbb{C}
$$

is symmetric (lemma 7.2) and nondegenerate. It is Grothendieck's residue pairing on $\Omega_{\mathbb{C}^{n+1}, 0}^{n+1} / d f \wedge \Omega_{\mathbb{C}^{n+1}, 0}^{n}$.

Relation to Steenbrink's Hodge filtration $F^{\bullet} H_{\mathbb{C}}^{\infty}:$ For $\lambda=e^{-2 \pi i \alpha}$ with $\alpha \in(-1,0]$,

$$
F_{S t}^{p} H_{\lambda}^{\infty}=\psi_{\alpha}^{-1}\left(\partial_{\tau}^{n-p} \operatorname{Gr}_{V}^{n-p+\alpha} H_{0}^{\prime \prime}(f)\right) .
$$

Define the unordered tuple $\operatorname{Sp}(f)=\sum_{i=1}^{\mu}\left(\alpha_{i}\right)=\sum_{\alpha \in \mathbb{Q}} d(\alpha) \cdot(\alpha) \in$ $\mathbb{Z}_{\geq 0}[\mathbb{Q}]$ of spectral numbers $\alpha_{1}, \ldots, \alpha_{\mu} \in \mathbb{Q}$ by

$$
d(\alpha):=\operatorname{dim} \mathrm{Gr}_{V}^{\alpha} H_{0}^{\prime \prime}-\operatorname{dim} \mathrm{Gr}_{V}^{\alpha} H_{0}^{\prime} .
$$

Number them such that $\alpha_{1} \leq \ldots \leq \alpha_{\mu}$. Then they satisfy the symmetry

$$
\alpha_{i}+\alpha_{\mu+1-i}=n-1
$$

and

$$
\begin{array}{r}
-1<\alpha_{1} \leq \ldots \leq \alpha_{\mu}<n, \\
V^{>-1} \supset H_{0}^{\prime \prime} \supset V^{n-1}, \\
0=F^{n+1} H^{\infty}, \quad F^{0} H_{\neq 1}^{\infty}=H_{\neq 1}^{\infty}, \quad F^{1} H_{1}^{\infty}=H_{1}^{\infty} .
\end{array}
$$

The algebraic properties had been proved by Brieskorn Br70] with some help by Sebastiani. That $K_{f}$ is the restriction to $H_{0}^{\prime \prime}(f)$ of $\mathrm{K}$. Saito's higher residue pairings [SaK83] follows from (7.21) and Pham's identification of $P$ with the Fourier-Laplace transform of K. Saito's higher residue pairings [Ph85]. See $[\mathrm{He} 02$ for an alternative reasoning. Then (7.25) and the properties of (7.26) follow from K. Saito's work.

Steenbrink defined the Hodge filtration $F_{S t}^{\bullet}$ first using resolution of singularities [St77]. Then Varchenko [Va80-1] constructed a closely related Hodge filtration $F_{V a}^{\bullet}$ from the Brieskorn lattice $H_{0}^{\prime \prime}(f)$. Scherk and Steenbrink SS85] (and also M. Saito) modified this construction 
to recover $F_{S t}^{\bullet}$. This is $(7.27)$. Then $(7.29)$ and $(7.30)$ follow from properties of the Hodge filtration. Though $V^{>-1} \supset H_{0}^{\prime \prime}$ was proved before by Malgrange.

Remark 7.8. The Fourier-Laplace transformation $F L$ is defined on any sum of elementary sections with the stronger convergence condition in (7.17). Therefore it is not defined on arbitrary elements of $H_{0}^{\prime \prime}$. But because of (7.30),

$$
H_{0}^{\prime \prime}=\left(H_{0}^{\prime \prime} \cap \bigoplus_{-1<\alpha<n-1} C_{\tau}^{\alpha}\right) \oplus V_{\tau}^{n-1},
$$

and the elements of the first summand are finite sums of elementary sections. Therefore the space

$$
F L\left(H_{0}^{\prime \prime} \cap \bigoplus_{-1<\alpha<n-1} C_{\tau}^{\alpha}\right) \oplus V_{z}^{n}
$$

is a well-defined free $\mathbb{C}\{z\}$-module of rank $\mu$. For simplicity we call it $F L\left(H_{0}^{\prime \prime}\right)$, although that is not completely correct. It satisfies

$$
\begin{aligned}
z^{2} \partial_{z}: F L\left(H_{0}^{\prime \prime}\right) & \rightarrow F L\left(H_{0}^{\prime \prime}\right), \\
\text { and } P: F L\left(H_{0}^{\prime \prime}\right) \times F L\left(H_{0}^{\prime \prime}\right) & \rightarrow z^{n+1} \cdot \mathbb{C}\{z\},
\end{aligned}
$$

and the leading part of $P$ is a symmetric and nondegenerate pairing on $F L\left(H_{0}^{\prime \prime}\right) / z \cdot F L\left(H_{0}^{\prime \prime}\right)$, all of this because of (7.19)-(7.21), (7.24)-(7.26). It thus satisfies all properties of a TERP-structure He02, definition 2.12]. Because of the $\mathbb{Z}$-lattice $H_{\mathbb{Z}}^{\infty}$ and the $\mathbb{Z}$-lattice bundle in the cohomology, we can even call it a TEZP-structure. More precisely, we denote as TEZP structure the following tuple.

$$
T E Z P(f):=\left(H_{\mathbb{Z}}^{\infty}, L^{n o r}, V_{z}^{\text {mod }}, P, F L\left(H_{0}^{\prime \prime}\right)\right)(f) .
$$

Here $V_{z}^{\text {mod }}$ comes equipped with the actions of $z, \partial_{z}^{-1}$ and $z \partial_{z}$. We formulated theorem 7.5 and introduced $F L\left(H_{0}^{\prime \prime}\right)$ because of the following Thom-Sebastiani result.

Theorem 7.9. SS85] BH17, Theorem 6.4] Consider besides $f\left(x_{0}, \ldots, x_{n}\right)$ a second singularity $g\left(x_{n+1}, \ldots, x_{n+m+1}\right)$. Then

$$
T E Z P(f+g) \cong T E Z P(f) \otimes T E Z P(g) .
$$

Remarks 7.10. (i) The isomorphism for the data $\left(H_{\mathbb{Z}}^{\infty}, L^{n o r}\right)$ is the classical Thom-Sebastiani result in (4.8) and (4.10). The isomorphism for $P$ follows from its definition with $L^{\text {nor }}$. The isomorphism for $V_{z}^{\text {mod }}$ is trivial. The isomorphism for $H_{0}^{\prime \prime}$ was essentially proved in [SS85, (8.7) Lemma]. Though Scherk and Steenbrink did not make the compatibility with the topological Thom-Sebastiani isomorphism between the cohomology bundles precise, and they avoided the use of 
the Fourier-Laplace transformation. They obtained a $\partial_{\tau}^{-1}$-linear isomorphism $H_{0}^{\prime \prime}(f+g) \cong H_{0}^{\prime \prime}(f) \otimes H_{0}^{\prime \prime}(g)$.

(ii) This is still fine. But then they mixed $\partial_{\tau}^{-1}$-linearity and $\tau$ linearity and went with this isomorphism directly into the defining formula 7.27 of $F_{S t}^{\bullet}$. This lead them to a wrong Thom-Sebastiani formula for $F_{S t}^{\bullet}$ in [SS85, Theorems (8.2) and (8.11)]. But the true Thom-Sebastiani formula is quite close [BH17, Corollary 6.5]. One has to replace in [SS85, Theorems (8.2) and (8.11)] $F_{S t}^{\bullet}$ by $G\left(F_{S t}^{\bullet}\right)$. This follows immediately from $(7.27)$ and $(7.36)$. Of course, in the case $N=0$, the isomorphism $G$ in definition 7.4 is just a rescaling, and then $G\left(F_{S t}^{\bullet}\right)=F_{S t}^{\bullet}$, so then their Thom-Sebastiani formula is correct.

(iii) As a corollary of theorem 7.9, we obtain for a suspension of $f$

$$
T E Z P\left(f+x_{n+1}^{2}\right) \cong T E Z P(f) \otimes T E Z P\left(x_{n+1}^{2}\right) \text {. }
$$

This allows us to consider in the sections 9 and 10 only the surface singularities. More generally, it implies the corollary 8.14. This corollary is the reason why we introduced $F L\left(H_{0}^{\prime \prime}(f)\right)$. Formula $(7.36)$ and this corollary are more elegant and general than the arguments with which suspensions were treated in [He93], [He95], [He11] and [GH17].

(iv) The Thom-Sebastiani formula for $F_{S t}^{\bullet}$ expresses in the case of a suspension $F_{S t}^{\bullet}\left(f+x_{n+1}^{2}\right)$ in terms of $F_{S t}^{\bullet}(f)$. It is made explicit in BH17, Theorem 4.6]. It can be seen as a square root of a Tate twist, because $F_{S t}^{\bullet}(f)$ and $F_{S t}^{\bullet}\left(f+x_{n+1}^{2}+x_{n+2}^{2}\right)$ are simply related by a Tate twist. $f$ and $f+x_{n+1}^{2}+x_{n+2}^{2}$ have the same polarizing form $S$ by (4.20) and 4.21), because $M_{h}(f)=M_{h}\left(f+x_{n+1}^{2}+x_{n+2}^{2}\right)$. But the polarizing form of $f+x_{n+1}^{2}$ is quite different, because of $M_{h}\left(f+x_{n+1}^{2}\right)=$ $-M_{h}(f)$ and (4.20) and 4.21). The formula in [BH17, Theorem 4.6] which expresses $F_{S t}^{\bullet}\left(f+x_{n+1}^{2}\right)$ in terms of $F_{S t}^{\bullet}(f)$ involves the $G^{(\alpha)}$ from definition 7.4 and is compatible with the isotropy condition (4.18) and (the generalization in the case $N \neq 0$ of) the positivity condition (4.19).

Fix for a moment a reference singularity $f_{0}$. In [He99] a classifying space $D_{P M H S}\left(f_{0}\right)$ and a classifying space $D_{B L}\left(f_{0}\right)$ are constructed. $D_{P M H S}$ is a classifying space for $M_{s}$-invariant Hodge filtrations $F^{\bullet}$ on $H_{\mathbb{C}}^{\infty}\left(f_{0}\right)$ such that $\left(H_{\neq 1}^{\infty}, H_{\neq 1, \mathbb{Z}}^{\infty}, F^{\bullet}, W,-N, S\right)$ and $\left(H_{1}^{\infty}, H_{1, \mathbb{Z}}^{\infty}, F^{\bullet}, W,-N, S\right)$ are polarized mixed Hodge structures of weight $n$ respectively $n+1$ with the same Hodge numbers as $F_{S t}^{\bullet}\left(f_{0}\right)$.

And $D_{B L}$ is a classifying space for subspaces $\mathcal{L}_{0} \subset V_{\tau}^{>-1}$ with the following properties:

$(\alpha) \mathcal{L}_{0}$ is a free $\mathbb{C}\{\tau\}$-module of rank $\mu$.

$(\beta) \mathcal{L}_{0}$ is a free $\mathbb{C}\left\{\left\{\partial_{\tau}^{-1}\right\}\right\}$-module of rank $\mu$. 
$(\gamma)$ The filtration $F^{\bullet}$ in $H_{\mathbb{C}}^{\infty}\left(f_{0}\right)$ which is constructed by formula (7.27) with $\mathcal{L}_{0}$ instead of $H_{0}^{\prime \prime}\left(f_{0}\right)$ is in $D_{P M H S}$.

( $\delta)$ It satisfies $K_{f}\left(\mathcal{L}_{0}, \mathcal{L}_{0}\right) \subset \partial_{\tau}^{-n-1} \cdot \mathbb{C}\left\{\left\{\partial_{\tau}^{-1}\right\}\right\}$.

Theorem 7.11. Fix a reference singularity $f\left(x_{0}, \ldots, x_{n}\right)$.

(a) [He99, ch. 2] $D_{P M H S}\left(f_{0}\right)$ is a real homogeneous space and a complex manifold. It is a locally trivial bundle over a product $D_{P H S}$ of classifying spaces for pure polarized Hodge structures. The fibers carry an affine algebraic structure and are isomorphic to $C^{N_{P M H S}}$ for some $N_{P M H S} \in \mathbb{Z}_{\geq 0}$. The group $G_{\mathbb{Z}}\left(f_{0}\right)$ acts properly discontinuously on $D_{P M H S}$.

(b) [He99, ch. 5] $D_{B L}\left(f_{0}\right)$ is a complex manifold and a locally trivial bundle over $D_{P M H S}$. The fibers have a natural $\mathbb{C}^{*}$-action with negative weights and are affine algebraic manifolds and are isomorphic to $\mathbb{C}^{N_{B L}}$ for some $N_{B L} \in \mathbb{Z}_{\geq 0}$. The group $G_{\mathbb{Z}}\left(f_{0}\right)$ acts properly discontinuously on $D_{B L}$.

(c) $D_{P M H S}\left(f_{0}\right)$ and $D_{P M H S}\left(f_{0}+x_{n+1}^{2}\right)$ are canonically isomorphic. $D_{B L}\left(f_{0}\right)$ and $D_{B L}\left(f_{0}+x_{n+1}^{2}\right)$ are canonically isomorphic.

Part (c) is not formulated in [He99]. The isomorphism $D_{B L}\left(f_{0}\right) \rightarrow$ $D_{B L}\left(f_{0}+x_{n+1}^{2}\right)$ is given by the generalization of $(7.36)$, namely the map

$$
\mathcal{L}_{0} \mapsto F L^{-1}\left(F L\left(\mathcal{L}_{0}\right) \otimes F L\left(H_{0}^{\prime \prime}\left(x_{n+1}^{2}\right)\right)\right) .
$$

The isomorphism $D_{P M H S}\left(f_{0}\right) \rightarrow D_{P M H S}\left(f_{0}+x_{n+1}^{2}\right)$ is obtained by applying $\mathrm{Gr}_{V}^{\bullet}$. It follows also from [BH17, Theorem 4.6].

In the sections 9 and $10, \mu$-constant families of singularities in two parameters will be studied. The following definition and theorem treat a more general situation. It had been considered especially in [Va80-2] [AGV88] SaM91] He93] Ku98].

Definition 7.12. A holomorphic $\mu$-constant family of singularities consists of a number $\mu \in \mathbb{Z}_{\geq 1}$, a complex manifold $T$, an open neighborhood $X \subset \mathbb{C}^{n+1} \times T$ of $\{0\} \times T$ and a holomorphic function $F: X \rightarrow \mathbb{C}$ such that $F_{t}:=\left.F\right|_{X_{t}}$ with $X_{t}:=X \cap \mathbb{C}^{n+1} \times\{t\}$ for any $t \in T$ has an isolated singularity at 0 with Milnor number $\mu$.

Theorem 7.13. Consider a holomorphic $\mu$-constant family as in definition 7.12.

(a) The Milnor lattices $\left(M l\left(F_{t}\right), L\right)$ with Seifert forms for $t \in T$ are locally canonically isomorphic. They glue to a local system $\bigcup_{t \in T} \operatorname{Ml}\left(F_{t}\right)$ of free $\mathbb{Z}$-modules of rank $\mu$. 
(b) Therefore also the spaces $C^{\alpha}\left(F_{t}\right), V_{\tau}^{\bmod }\left(F_{t}\right), V_{\tau}^{\alpha}\left(F_{t}\right)$ are locally canonically isomorphic and glue to local systems.

(c) But the Brieskorn lattices $H_{0}^{\prime \prime}\left(F_{t}\right) \subset V_{\tau}^{>-1}\left(F_{t}\right)$ vary holomorphically. For $\omega \in \Omega_{X / T}^{n+1}, s[\omega]_{0}(t):=s\left[\left.\omega\right|_{X_{t}}\right]_{0} \in H_{0}^{\prime \prime}\left(F_{t}\right)$. Let $\xi$ be a holomorphic vector field on $T$. Its canonical lifts to $\mathbb{C} \times T$ (with coordinate $\tau$ on $\mathbb{C}$ ) and $X$ are also denoted $\xi$. The covariant derivative of $s[\omega]_{0}(t)$ by $\xi$ is

$$
\xi s[\omega]_{0}(t)=s\left[\operatorname{Lie}_{\xi} \omega\right]_{0}(t)+\left(-\partial_{\tau}\right) s[\xi(F) \cdot \omega]_{0}(t) .
$$

(d) All germs $F_{t}$ have the same spectrum.

Remarks 7.14. (i) Part (a) is less trivial than one might expect, as it is not clear whether $\varepsilon(t)$ and $\delta(t)$ in the definition of a Milnor fibration $F_{t}: X(\varepsilon(t), \delta(t)) \rightarrow \Delta_{\delta(t)}$ can be chosen as continuous functions in t. But lemma 2.2 in [LR73] saves the situation. See [Va80-2] He93] [Ku98] He11] for details.

(ii) Part (b) follows from part (a). Formula (7.39) is well known, see e.g. [Va80-2] AGV88] He93] [Ku98]. Part (d) is proved in [Va82].

(iii) The bundle $\bigcup_{t \in T} H_{0}^{\prime \prime}\left(F_{t}\right) \subset \bigcup_{t \in T} V_{\tau}^{>-1}\left(F_{t}\right)$ can be seen as a germ along $\{0\} \times T$ on $(\mathbb{C}, 0) \times T$ of a holomorphic rank $\mu$ bundle.

$s[\omega]_{0}$ for $\omega \in \Omega_{X / T}^{n+1}$ is a holomorphic section in this bundle.

But in theorem 9.6 and theorem 10.6 we will be imprecise and consider $s[\omega]_{0}$ as a possibly multi-valued holomorphic map $s[\omega]_{0}: T \rightarrow$ $V_{\tau}^{>-1}\left(F_{t^{0}}\right)$ for a reference singularity $F_{t^{0}}$.

(iv) $s[\omega]_{0}$ is a sum $s[\omega]_{0}=\sum_{\alpha>-1} s(\omega, \alpha)$ of holomorphic families $s(\omega, \alpha)(t) \in C^{\alpha}\left(F_{t}\right), t \in T$, of elementary sections. For each $t \in T$,

$$
\alpha\left(s[\omega]_{0}(t)\right):=\alpha\left(\left.\omega\right|_{X_{t}}\right):=\min (\alpha \mid s(\omega, \alpha)(t) \neq 0)
$$

is the order of $s[\omega]_{0}(t)$, and $s\left(\omega, \alpha\left(\left.\omega\right|_{X_{t}}\right)\right)(t)$ is its principal part. The order is upper semicontinuous in $t$.

(v) A notation: $\omega_{0}:=d x_{0} \ldots d x_{n}$.

All bimodal series singularities in table 9.1 except $W_{1, p}^{\sharp}$ (see remark 9.5 for $W_{1, p}^{\sharp}$ ) are Newton nondegenerate. All quadrangle singularities in table (10.1) are semiquasihomogeneous. For such singularities there are useful results for the computation of the order $\alpha\left(\left.\omega\right|_{X_{t}}\right)$, which we describe in the following. We start with a definition of Kouchnirenko.

Definition 7.15. Let $f:\left(\mathbb{C}^{n+1}, 0\right) \rightarrow(\mathbb{C}, 0)$ be a singularity. 
(a) Ko76] Write $f=\sum_{i \in \mathbb{Z}_{\geq 0}^{n+1}} a_{i} x^{i}$ and define

$$
\begin{aligned}
\operatorname{supp}(f) & :=\left\{i \in \mathbb{Z}_{\geq 0}^{n+1} \mid a_{i} \neq 0\right\} \\
\Gamma_{+}(f) & :=\left(\text { convex hull of } \bigcup_{i \in \operatorname{supp}(f)}\left(i+\mathbb{R}_{\geq 0}^{n+1}\right)\right) \subset \mathbb{R}^{n+1}, \\
\Gamma_{\text {com }}(f) & :=\left\{\sigma \mid \sigma \text { is a compact face of } \Gamma_{+}(f)\right\}, \\
\Gamma_{\text {com }, n}(f) & :=\left\{\sigma \in \Gamma_{\text {com }}(f) \mid \operatorname{dim} \sigma=n\right\} \\
l_{\sigma} & : \mathbb{R}^{n+1} \rightarrow \mathbb{R} \text { for } \sigma \in \Gamma_{\text {com }, n}(f)
\end{aligned}
$$

as the linear function with $\sigma \subset l_{\sigma}^{-1}(1)$.

(b) SaM88] [KV85] The Newton order $\nu: \mathbb{C}\left\{x_{0}, \ldots, x_{n}\right\} \rightarrow \mathbb{Q}_{\geq 0} \cup$ $\{\infty\}$ is

$$
\nu\left(\sum_{i} b_{i} x^{i}\right):=\min \left(l_{\sigma}(i) \mid \text { all } i \text { with } b_{i} \neq 0, \text { all } \sigma \in \Gamma_{\text {com }, n}(f)\right\} .
$$

The Newton order $\nu: \Omega_{\mathbb{C}^{n+1,0}}^{n+1} \rightarrow \mathbb{Q}_{>0} \cup\{\infty\}$ is

$$
\nu\left(\left(\sum_{i} b_{i} x^{i}\right) \cdot \omega_{0}\right):=\nu\left(\left(\sum_{i} b_{i} x^{i}\right) x_{0} \ldots x_{n}\right) .
$$

The Newton order $\bar{\nu}: H_{0}^{\prime \prime}(f) \rightarrow \mathbb{Q}_{>0} \cup\{\infty\}$ is

$$
\bar{\nu}:=\max \left(\nu(\eta) \mid \eta \equiv \omega \quad \bmod d f \wedge d \Omega_{\mathbb{C}^{n+1}, 0}^{n-1}\right) .
$$

(c) [Ko76] For $\sigma \in \Gamma_{\text {com }}(f)$ define $f_{\sigma}:=\sum_{i \in \sigma} a_{i} x^{i}$. The singularity $f$ is Newton nondegenerate if for each $\sigma \in \Gamma_{\text {com }}(f)$ the Jacobi ideal $J\left(f_{\sigma}\right)$ of $f_{\sigma}$ has no zero in $\left(\mathbb{C}^{*}\right)^{n+1}$. It is convenient if $f$ contains for each index $j \in\{0, \ldots, n\}$ a monomial $x_{j}^{m_{j}}$ for some $m_{j} \geq 2$.

The following theorem was proved in 1983 by M. Saito SaM88. The proof shortly afterwards by Khovanskii and Varchenko KV85] is completely different.

Theorem 7.16. Let $f$ be a Newton nondegenerate and convenient singularity. For any $\omega \in \Omega_{\mathbb{C}^{n+1}, 0}^{n+1}$, its order $\alpha(\omega)$ (defined in remark 7.14 (iv)) is $\alpha(\omega)=\bar{\nu}(\omega)-1$.

The following corollary is an easy consequence. It is proved in [He93, Satz 1.10].

Corollary 7.17. Let $f$ be a Newton nondegenerate and convenient singularity. Define

$$
\begin{aligned}
s(f) & :=\min \left(\nu\left(\frac{\partial f}{\partial x_{j}} \cdot \omega_{0}\right)-1 \mid j \in\{0, \ldots, n\}\right)>0, \\
I(f) & :=\left\{i \in \mathbb{Z}_{\geq 0}^{n+1} \mid \nu\left(x^{i} \omega_{0}\right)-1<s(f)\right\} .
\end{aligned}
$$


Then for $i \in I(f)$

$$
\alpha\left(x^{i} \omega_{0}\right)=\nu\left(x^{i} \omega_{0}\right)-1,
$$

the numbers $\alpha\left(x^{i} \omega_{0}\right), i \in I(f)$, are the spectral numbers in the interval $(-1, s(f))$, and

$$
\alpha\left(\left(\sum_{i} b_{i} x^{i}\right) \cdot \omega_{0}\right)=\left\{\begin{array}{c}
\min \left(\alpha\left(x^{i} \omega_{0}\right) \mid i \in I(f), b_{i} \neq 0\right) \\
\text { if an } i \in I(f) \text { with } b_{i} \neq 0 \text { exists } \\
\geq s(f) \quad \text { else. }
\end{array}\right.
$$

Remarks 7.18. (i) We expect that theorem 7.16 holds also without the condition that $f$ is convenient. This would be desirable as many normal forms of singularities are Newton nondegenerate, but not convenient.

(ii) A singularity is $(\mu+1)$-determined, i.e. $f+g \sim_{\mathcal{R}} f$ for any $g \in \mathbf{m}^{\mu+1}$, where $\mathbf{m}$ is the maximal ideal in $\mathbb{C}\{x\}$ Ma68. If $f$ is Newton nondegenerate, then $f+\sum_{j=0}^{n} c_{j} x_{j}^{m_{j}}$ for arbitrary $m_{j} \geq \mu+1$ and sufficiently generic $c_{j} \in \mathbb{C}^{*}$ is Newton nondegenerate and convenient and right equivalent to $f$.

Furthermore, because of $\mathbf{m}^{\mu} \subset J(f)$ and the Artin approximation theorem, one can choose a coordinate change $\varphi$ with $f+\sum_{j=0}^{n} c_{j} x_{j}^{m_{j}}=$ $f \circ \varphi$ such that all $\varphi_{j}-x_{j} \in \mathbf{m}^{\min \left(m_{k}\right)-\mu}$. Unfortunately, this is not sufficient for a generalization of theorem 7.16 to the case where $f$ is not convenient.

(iii) We claim that the calculations in the proof of theorem 9.6 can be carried out with almost no change (but with additional terms) for $f+\sum_{j=0}^{n} c_{j} x_{j}^{m_{j}}$ with large $m_{j}$ and that they give essentially the same results. With this claim, we justify that we calculate in the proof of theorem 9.6 with the normal forms $f$ in table (9.1) which are almost all not convenient, but that we apply theorem 7.16 and corollary 7.17 .

(iv) Theorem 7.16 holds without the condition that $f$ is convenient if $f$ is semiquasihomogeneous. That is the case when there is only one compact face of dimension $n$.

Definition 7.19. (a) A singularity $f$ is semiquasihomogeneous with weights $w_{0}, \ldots, w_{n} \in \mathbb{Q}_{>0}$ if

$$
f=\sum_{i \in \mathbb{Z}_{\geq 0}^{n+1}} a_{i} x^{i} \text { with } \operatorname{deg}_{w} x^{i} \geq 1 \text { for all } i \text { with } a_{i} \neq 0,
$$

and the quasihomogeneous polynomial

$$
f_{q h}:=\sum_{i: \operatorname{deg}_{w} x^{i}=1} a_{i} x^{i}
$$


has an isolated singularity at 0 .

(b) A singularity $f$ is quasihomogeneous if it is semiquasihomogeneous with $f=f_{q h}$.

A quasihomogeneous singularity $f$ satisfies the Euler equation

$$
f=\sum_{j=0}^{n} w_{j} x_{j} \frac{\partial f}{\partial x_{j}} .
$$

This equation and (7.24) and elementary calculations in Br70] imply part (a) of the following lemma.

Lemma 7.20. (a) Let $f$ be a quasihomogeneous singularity with weights $\left(w_{0}, \ldots, w_{n}\right)$. If $\omega=x^{i} \omega_{0}$ is a monomial differential form then

either $\quad s[\omega]_{0}=0$

$$
\text { or } \quad \alpha(\omega)=\operatorname{deg}_{w}\left(x^{i} x_{0} \ldots x_{n}\right)-1 \text { and } s[\omega]_{0}=s(\omega, \alpha(\omega)) .
$$

(b) Let $f$ be a semiquasihomogeneous singularity with weights $\left(w_{0}, \ldots, w_{n}\right)$ and $f \neq f_{q h}$. The 1-parameter family $f_{q h}+t \cdot\left(f-f_{q h}\right)$ is a $\mu$-constant family. If $\omega=x^{i} \omega_{0}$ is a monomial differential form then

$$
\begin{aligned}
\alpha(\omega) & \geq \operatorname{deg}_{w}\left(x^{i} x_{0} \ldots x_{n}\right)-1, \\
s(\omega & \left., \quad \operatorname{deg}_{w}\left(x^{i} x_{0} \ldots x_{n}\right)-1\right)(t)=s[\omega]_{0}(0), \\
s(\omega, \alpha)(t) & =\sum_{k \geq 0} \frac{1}{k !} \cdot t^{k} \cdot\left(-\partial_{\tau}\right)^{k} s\left(\left(f-f_{q h}\right)^{k} \cdot \omega, \alpha+k\right)(0) .
\end{aligned}
$$

The last expression is polynomial in $t$ because $\alpha\left(\left(f-f_{q h}\right)^{k} \omega\right)>\alpha+k$ for large $k$.

Proof of part (b): In [AGV85, ch. 12] it is shown that $f_{q h}+t(f-$ $\left.f_{q h}\right)$ is a $\mu$-constant family. The other assertions follow with theorem 7.13 (c) and part (a) of lemma 7.20.

8. REVIEW ON MARKED SINGULARITIES, THEIR MODULI SPACES, $\mu$-CONSTANT MONODROMY GROUPS AND TORELLI CONJECTURES

This paper and the paper [GH17] complete the study of the data in the title of this section for the singularities of modality $\leq 2$. These data were introduced in [He11]. Here we review them. We start with the notions marked singularity and strongly marked singularity.

Definition 8.1. Fix one reference singularity $f_{0}$.

(a) Then a strong marking for any singularity $f$ in the $\mu$-homotopy class of $f_{0}$ (i.e. there is a 1-parameter family of singularities with 
constant Milnor number connecting $f$ and $f_{0}$ ) is an isomorphism $\rho$ : $(M l(f), L) \rightarrow\left(M l\left(f_{0}\right), L\right)$.

(b) The pair $(f, \rho)$ is a strongly marked singularity. Two strongly marked singularities $\left(f_{1}, \rho_{1}\right)$ and $\left(f_{2}, \rho_{2}\right)$ are right equivalent (notation: $\left.\sim_{\mathcal{R}}\right)$ if a coordinate change $\varphi:\left(\mathbb{C}^{n+1}, 0\right) \rightarrow\left(\mathbb{C}^{n+1}, 0\right)$ with

$$
f_{1}=f_{2} \circ \varphi \text { and } \rho_{1}=\rho_{2} \circ \varphi_{\text {hom }}
$$

exists, where $\varphi_{\text {hom }}:\left(M l\left(f_{1}\right), L\right) \rightarrow\left(M l\left(f_{2}\right), L\right)$ is the induced isomorphism.

(c) The notion of a marked singularity is slightly weaker. If $f$ and $\rho$ are as above, then the pair $(f, \pm \rho)$ is a marked singularity (writing $\pm \rho$, the set $\{\rho,-\rho\}$ is meant, neither $\rho$ nor $-\rho$ is preferred).

(d) Two marked singularities $\left(f_{1}, \pm \rho_{1}\right)$ and $\left(f_{2}, \pm \rho_{2}\right)$ are right equivalent (notation: $\sim_{\mathcal{R}}$ ) if a coordinate change $\varphi$ with

$$
f_{1}=f_{2} \circ \varphi \quad \text { and } \quad \rho_{1}=\varepsilon \rho_{2} \circ \varphi_{\text {hom }} \quad \text { for some } \varepsilon \in\{ \pm 1\}
$$

exists.

Remarks 8.2. (i) The notion of a marked singularity behaves better than the notion of a strongly marked singularity, because it is not known whether all $\mu$-homotopy families of singularities satisfy one of the following two properties:

$$
\begin{array}{ll}
\text { Assumption (8.1): } & \begin{array}{l}
\text { Any singularity in the } \mu \text {-homotopy } \\
\text { class of } f_{0} \text { has multiplicity } \geq 3 .
\end{array} \\
\text { Assumption (8.2): } & \begin{array}{l}
\text { Any singularity in the } \mu \text {-homotopy } \\
\text { class of } f_{0} \text { has multiplicity } 2 .
\end{array}
\end{array}
$$

We expect that always one of two assumptions holds. For curve singularities and singularities right equivalent to semiquasihomogeneous singularities and all singularities with modality $\leq 2$ this is true, but in general it is not known. In a $\mu$-homotopy family where neither of the two assumptions holds, strong marking behaves badly, see (ii).

(ii) If mult $(f)=2$ then $(f, \rho) \sim_{\mathcal{R}}(f,-\rho)$, which is easy to see. If mult $(f) \geq 3$, then $(f, \rho) \chi_{\mathcal{R}}(f,-\rho)$, whose proof in [He11] is quite intricate. These properties imply that the moduli space for strongly marked singularities discussed below is not Hausdorff in the case of a $\mu$-homotopy class which satisfies neither one of the assumptions (8.1) or 8.2 .

In He02 a moduli space $M_{\mu}\left(f_{0}\right)$ was constructed for the $\mu$-homotopy class of any singularity $f_{0}$. As a set it is simply the set of right equivalence classes of singularities in the $\mu$-homotopy class of $f_{0}$. But in 
He02 it is constructed as an analytic geometric quotient, and it is shown that it is locally isomorphic to the $\mu$-constant stratum of a singularity modulo the action of a finite group. The $\mu$-constant stratum of a singularity is the germ $\left(S_{\mu}, 0\right) \subset(M, 0)$ within the germ of the base space of a universal unfolding $F$ of $f$, such that for a suitable representative

$$
S_{\mu}=\left\{t \in M \mid F_{t} \text { has only one singularity } x_{0} \text { and } F_{t}\left(x_{0}\right)=0\right\} .
$$

It comes equipped with a canonical complex structure, and $M_{\mu}$ inherits a canonical complex structure, see the chapters 12 and 13 in [He02].

In [He11 analogous results for marked singularities were proved. A better property is that $M_{\mu}^{\text {mar }}$ is locally isomorphic to a $\mu$-constant stratum without dividing out a finite group action. Therefore one can consider it as a global $\mu$-constant stratum or as a Teichmüller space for singularities. The following theorem collects results from He11, theorem 4.3].

Theorem 8.3. Fix one reference singularity $f_{0}$. Define the sets

$$
\begin{aligned}
M_{\mu}^{\text {smar }}\left(f_{0}\right):= & \{\text { strongly marked }(f, \rho) \mid \\
& \left.f \text { in the } \mu \text {-homotopy class of } f_{0}\right\} / \sim_{\mathcal{R}}, \\
M_{\mu}^{\text {mar }}\left(f_{0}\right):= & \{\text { marked }(f, \pm \rho) \mid \\
& \left.f \text { in the } \mu \text {-homotopy class of } f_{0}\right\} / \sim_{\mathcal{R}} .
\end{aligned}
$$

(a) $M_{\mu}^{\text {mar }}\left(f_{0}\right)$ carries a natural canonical complex structure. It can be constructed with the underlying reduced complex structure as an analytic geometric quotient (see [He11, theorem 4.3] for details).

(b) The germ $\left(M_{\mu}^{\text {mar }}\left(f_{0}\right),[(f, \pm \rho)]\right)$ with its canonical complex structure is isomorphic to the $\mu$-constant stratum of $f$ with its canonical complex structure (see [He02, chapter 12] for the definition of that).

(c) For any $\psi \in G_{\mathbb{Z}}\left(f_{0}\right)=: G_{\mathbb{Z}}$, the map

$$
\psi_{\text {mar }}: M_{\mu}^{\text {mar }} \rightarrow M_{\mu}^{\text {mar }}, \quad[(f, \pm \rho)] \rightarrow[(f, \pm \psi \circ \rho)]
$$

is an automorphism of $M_{\mu}^{\text {mar }}$. The action

$$
G_{\mathbb{Z}} \times M_{\mu}^{\text {mar }} \rightarrow M_{\mu}^{\text {mar }}, \quad\left(\psi,[(f, \pm \rho)] \mapsto \psi_{\text {mar }}([(f, \pm \rho)])\right.
$$

is a group action from the left.

(d) The action of $G_{\mathbb{Z}}$ on $M_{\mu}^{\text {mar }}$ is properly discontinuous. The quotient $M_{\mu}^{\text {mar }} / G_{\mathbb{Z}}$ is the moduli space $M_{\mu}$ for right equivalence classes in the $\mu$-homotopy class of $f_{0}$, with its canonical complex structure. Especially, $\left[\left(f_{1}, \pm \rho_{1}\right)\right]$ and $\left[\left(f_{2}, \pm \rho_{2}\right)\right]$ are in one $G_{\mathbb{Z}^{-}}$orbit if and only if $f_{1}$ and $f_{2}$ are right equivalent. 
(e) If assumption (8.1) or (8.2) holds then (a) to (d) are also true for $M_{\mu}^{\text {smar }}$ and $\psi_{\text {smar }}$ with $\psi_{\text {smar }}([(f, \rho)]):=[(f, \psi \circ \rho)]$. If neither (8.1) nor (8.2) holds then the natural topology on $M_{\mu}^{\text {smar }}$ is not Hausdorff.

We stick to the situation in theorem 8.3 and define two subgroups of $G_{\mathbb{Z}}\left(f_{0}\right)$. The definitions in [He11, definition 3.1] are different, they use $\mu$-constant families. The following definitions are a part of theorem 4.4 in [He11].

Definition 8.4. Let $\left(M_{\mu}^{\text {mar }}\right)^{0}$ be the topological component of $M_{\mu}^{\text {mar }}$ (with its reduced complex structure) which contains $\left[\left(f_{0}, \pm \mathrm{id}\right)\right]$. Then

$$
G^{\text {mar }}\left(f_{0}\right):=\left\{\psi \in G_{\mathbb{Z}} \mid \psi \text { maps }\left(M_{\mu}^{\text {mar }}\right)^{0} \text { to itself }\right\} \subset G_{\mathbb{Z}}\left(f_{0}\right)(8.6)
$$

If assumption (8.1) or 8.2 holds, $\left(M_{\mu}^{\text {smar }}\right)^{0}$ and $G^{\text {smar }}\left(f_{0}\right) \subset G_{\mathbb{Z}}\left(f_{0}\right)$ are defined analogously.

The following theorem is also proved in [He11].

Theorem 8.5. (a) In the situation above, the map

$$
\begin{aligned}
G_{\mathbb{Z}} / G^{\text {mar }}\left(f_{0}\right) & \left.\rightarrow \text { \{topological components of } M_{\mu}^{\text {mar }}\right\} \\
\psi \cdot G^{\text {mar }}\left(f_{0}\right) & \mapsto \text { the component } \psi_{\text {mar }}\left(\left(M_{\mu}^{\text {mar }}\right)^{0}\right)
\end{aligned}
$$

is a bijection.

(b) If assumption (8.1) or (8.2) holds then (a) is also true for $M_{\mu}^{\text {smar }}$ and $G^{\text {smar }}\left(f_{0}\right)$.

(c) $-\mathrm{id} \in G_{\mathbb{Z}}$ acts trivially on $M_{\mu}^{\text {mar }}\left(f_{0}\right)$. Suppose that assumption (8.2) holds and that $f_{0}=g_{0}\left(x_{0}, \ldots, x_{n-1}\right)+x_{n}^{2}$. Then $-\mathrm{id}$ acts trivially on $M_{\mu}^{\text {smar }}\left(f_{0}\right)$ and

$$
\begin{aligned}
& M_{\mu}^{\text {smar }}\left(f_{0}\right)=M_{\mu}^{\text {mar }}\left(f_{0}\right)=M_{\mu}^{\text {mar }}\left(g_{0}\right), \\
& G^{\text {smar }}\left(f_{0}\right)=G^{\text {mar }}\left(f_{0}\right)=G^{\text {mar }}\left(g_{0}\right) .
\end{aligned}
$$

Suppose additionally that assumption (8.1) holds for $g_{0}$ (instead of $f_{0}$ ). Then $\{ \pm \mathrm{id}\}$ acts freely on $M_{\mu}^{\text {smar }}\left(g_{0}\right)$, and the quotient map

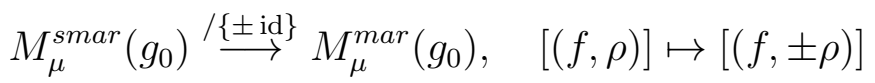

is a double covering.

The following conjecture was formulated as conjecture 3.2 in [He11].

Conjecture 8.6. [He11, Conjecture 3.2] (a) Fix a singularity $f_{0}$. Then $M_{\mu}^{\text {mar }}$ is connected. Equivalently (in view of theorem 8.5 (a)): $G^{\operatorname{mar}}\left(f_{0}\right)=G_{\mathbb{Z}}$.

(b) If the $\mu$-homotopy class of $f_{0}$ satisfies assumption (8.1), then - id $\notin G^{s m a r}\left(f_{0}\right)$. 
The study of the singularities with modality $\leq 2$ in [He11 GH17 and this paper gives: Part (b) is true for all singularities with modality $\leq 2$. Part (a) is true for almost all singularities with modality $\leq 2$, but not for all. The exceptions are the subseries for $p=m \cdot r$ of the eight bimodal series. This is a part of theorem 9.1. Now we expect that part (a) will be wrong for many singularities.

Using the other definition of $G^{\text {mar }}$ in [He11, part (a) says that up to $\pm \mathrm{id}$, any element of $G_{\mathbb{Z}}$ can be realized as transversal monodromy of a $\mu$-constant family with parameter space $S^{1}$. As it is wrong for some singularities and probably for many more, part (a) of conjecture 8.6 has to be replaced now by the question whether the subgroup $G^{\text {mar }}$ of $G_{\mathbb{Z}}$ can be described in a nice conceptual way.

In order to understand the stabilizers $\operatorname{Stab}_{G_{\mathbb{Z}}}([(f, \rho)])$ and $\operatorname{Stab}_{G_{\mathbb{Z}}}([(f, \pm \rho)])$ of points $[(f, \rho)] \in M_{\mu}^{\text {smar }}\left(f_{0}\right)$ and $[(f, \pm \rho)] \in$ $M_{\mu}^{\text {mar }}\left(f_{0}\right)$, we have to look at the symmetries of a single singularity. These had been discussed in [He02, chapter 13.2]. The discussion had been taken up again in [He11].

Definition 8.7. Let $f_{0}=f_{0}\left(x_{0}, \ldots, x_{n}\right)$ be a reference singularity and let $f$ be any singularity in the $\mu$-homotopy class of $f_{0}$. If $\rho$ is a marking, then $G_{\mathbb{Z}}(f)=\rho^{-1} \circ G_{\mathbb{Z}} \circ \rho$.

We define

$$
\begin{aligned}
\mathcal{R} & :=\left\{\varphi:\left(\mathbb{C}^{n+1}, 0\right) \rightarrow\left(\mathbb{C}^{n+1}, 0\right) \text { biholomorphic }\right\}(8.8) \\
\mathcal{R}^{f} & :=\{\varphi \in \mathcal{R} \mid f \circ \varphi=f\}, \\
R_{f} & :=j_{1} \mathcal{R}^{f} /\left(j_{1} \mathcal{R}^{f}\right)^{0}, \\
G_{\mathcal{R}}^{\text {smar }}(f) & :=\left\{\varphi_{\text {hom }} \mid \varphi \in \mathcal{R}^{f}\right\} \subset G_{\mathbb{Z}}(f), \\
G_{\mathcal{R}}^{\text {mar }}(f) & :=\left\{ \pm \psi \mid \psi \in G_{\mathcal{R}}^{\text {smar }}(f)\right\}, \\
G_{\mathcal{R}}^{\text {smar, gen }}\left(f_{0}\right) & :=\bigcap_{[(f, \rho)] \in M_{\mu}^{\text {smar }}} \rho^{-1} \circ G_{\mathcal{R}}^{\text {smar }}(f) \circ \rho \subset G_{\mathbb{Z}} .
\end{aligned}
$$

Again, the definition of $G_{\mathcal{R}}^{\text {smar }}$ is different from the definition in [He11, definition 3.1]. The characterization in 8.11) is [He11, theorem 3.3. (e)]. $R_{f}$ is the finite group of components of the group $j_{1} \mathcal{R}^{f}$ of 1 -jets of coordinate changes which leave $f$ invariant. The following theorem collects results from several theorems in [He11.

Theorem 8.8. Consider the data in definition 8.7.

(a) If mult $(f) \geq 3$ then $j_{1} \mathcal{R}^{f}=R_{f}$.

(b) The homomorphism ()$_{\text {hom }}: \mathcal{R}^{f} \rightarrow G_{\mathbb{Z}}(f)$ factors through $R_{f}$. Its image is $\left(R_{f}\right)_{\text {hom }}=G_{\mathcal{R}}^{\text {smar }}(f) \subset G_{\mathbb{Z}}(f)$.

(c) The homomorphism ()$_{\text {hom }}: R_{f} \rightarrow G_{\mathcal{R}}^{\text {smar }}(f)$ is an isomorphism. 
(d)

$$
-\mathrm{id} \notin G_{\mathcal{R}}^{\text {smar }}(f) \Longleftrightarrow \operatorname{mult} f \geq 3 \text {. }
$$

Equivalently: $G_{\mathcal{R}}^{\text {mar }}(f)=G_{\mathcal{R}}^{\text {smar }}(f)$ if mult $f=2$, and $G_{\mathcal{R}}^{\text {mar }}(f)=$ $G_{\mathcal{R}}^{\text {smar }}(f) \times\{ \pm$ id $\}$ if mult $f \geq 3$.

(e) $G_{\mathcal{R}}^{\operatorname{mar}}(f)=G_{\mathcal{R}}^{\text {mar }}\left(f+x_{n+1}^{2}\right)$.

(f) $M_{h} \in G^{\text {smar }}(f)$. If $f$ is quasihomogeneous then $M_{h} \in G_{\mathcal{R}}^{\text {smar }}(f)$.

(g) For any $[(f, \rho)] \in M_{\mu}^{\text {smar }}$

$$
\begin{aligned}
\operatorname{Stab}_{G_{\mathbb{Z}}}([(f, \rho)]) & =\rho \circ G_{\mathcal{R}}^{\text {smar }}(f) \circ \rho^{-1}, \\
\operatorname{Stab}_{G_{\mathbb{Z}}}([(f, \pm \rho)]) & =\rho \circ G_{\mathcal{R}}^{\text {mar }}(f) \circ \rho^{-1} .
\end{aligned}
$$

( (8.15) does not require assumption (8.1) or (8.2)). As $G_{\mathbb{Z}}$ acts properly discontinuously on $M_{\mu}^{\text {mar }}\left(f_{0}\right), G_{\mathcal{R}}^{\text {smar }}(f)$ and $G_{\mathcal{R}}^{\text {mar }}(f)$ are finite. (But this follows already from the finiteness of $R_{f}$ and (b).)

The group $G_{\mathcal{R}}^{\text {smar,gen }}\left(f_{0}\right)$ in 8.13 had not been considered in [He11. Usually it is very small. It is useful because of the following elementary fact.

Lemma 8.9. Let $T$ be the parameter space of a $\mu$-constant family as in definition 7.12. The transversal monodromy of it is the representation $\pi_{1}\left(T, t^{0}\right) \rightarrow G_{\mathbb{Z}}\left(F_{t^{0}}\right)$ which comes from the local system $\bigcup_{t \in T} \operatorname{Ml}\left(F_{t}\right)$.

If its image is in $G_{\mathcal{R}}^{\text {smar,gen }}\left(F_{t^{0}}\right)$, then there is a natural map $T \rightarrow$ $M_{\mu}^{\text {smar }}\left(F_{t^{0}}\right)$.

Proof: The trivial strong marking + id for $F_{t^{0}}$ induces along any path strong markings of other singularities $F_{t}$. Two paths which meet at a point $t$, might not induce the same strong marking of $F_{t}$, but the two markings differ only by an element of $G_{\mathcal{R}}^{\text {smar }}\left(F_{t}\right)$. Therefore they induce the same right equivalence class of a marked singularity.

Finally, we come to the Brieskorn lattices of marked singularities and Torelli problems. After fixing a reference singularity $f_{0}$, a marked singularity $(f, \pm \rho)$ comes equipped with a marked Brieskorn lattice $B L(f, \pm \rho)$. The classifying space $D_{B L}\left(f_{0}\right)$ in theorem 7.11 is a classifying space for marked Brieskorn lattices. Theorem 7.13 implies part (a) of the following theorem.

Theorem 8.10. Fix one reference singularity $f_{0}$.

(a) There is a natural holomorphic period map

$$
B L: M_{\mu}^{\operatorname{mar}}\left(f_{0}\right) \rightarrow D_{B L}\left(f_{0}\right) .
$$

It is $G_{\mathbb{Z}}$-equivariant.

(b) [He02, theorem 12.8] It is an immersion, here the reduced complex structure on $M_{\mu}^{\text {mar }}\left(f_{0}\right)$ is considered. 
The second author conjectured part (b) of the following global Torelli conjecture in [He93], part (c) in [He02] and part (a) in [He11].

Conjecture 8.11. Fix one reference singularity $f_{0}$.

(a) The period map $B L: M_{\mu}^{\text {mar }} \rightarrow D_{B L}$ is injective.

(b) The period map LBL: $M_{\mu}=M_{\mu}^{\text {mar }} / G_{\mathbb{Z}} \rightarrow D_{B L} / G_{\mathbb{Z}}$ is injective.

(c) For any singularity $f$ in the $\mu$-homotopy class of $f_{0}$ and any marking $\rho$,

$$
\operatorname{Stab}_{G_{\mathbb{Z}}}([(f, \pm \rho)])=\operatorname{Stab}_{G_{\mathbb{Z}}}(B L([(f, \pm \rho)]))
$$

(only $\subset$ and the finiteness of both groups are clear).

The second author has a long-going project on Torelli type conjectures. Already in He93, part (b) was proved for all simple and unimodal singularities and almost all bimodal singularities (all except 3 subseries of the 8 bimodal series). This was possible without the general construction of $M_{\mu}$ and $D_{B L}$, which came later in [He02] and [He99]. In the concrete cases considered in [He93], it is easy to identify a posteriori the spaces $M_{\mu}$ and $D_{B L}$.

The following lemma from He11 clarifies the logic between the parts (a), (b) and (c) of conjecture 8.11.

Lemma 8.12. In conjecture 8.11, (a) $\Longleftrightarrow$ (b) and (c).

Part (a) of conjecture 8.11 was proved in [He11] for the simple and those 22 of the 28 exceptional unimodal and bimodal singularities, where all eigenvalues of the monodromy have multiplicity one. In GH17 part (a) was proved for the remaining unimodal and the remaining exceptional bimodal singularities. In the sections 9 and 10 , part (a) will be proved for the remaining bimodal singularities, namely the bimodal series singularities and the quadrangle singularities.

As part (b) had been proved for almost all singularities with modality $\leq 2$, the main work in GH17] and here is the good control of the group $G_{\mathbb{Z}}$. But that is surprisingly difficult. In the case of the bimodal singularities in this paper, also the control of the Gauss-Manin connection side had to be improved: We provide better information on the transversal monodromy of the studied families than in [He93]. Due to this improvement, also the annoying gap of 3 subseries of the 8 bimodal series, where part (b) was not proved in [He93, could be closed here.

Remark 8.13. In the sections 9 and 10 , we will restrict to consider surface singularities, i.e. singularities in 3 variables. This is justified by the following corollary. It is an application for suspensions of the Thom-Sebastiani formula for the Fourier-Laplace transforms of Brieskorn lattices in theorem 7.9. This is elegant, but the preparations 
in section 7 were heavy. In the earlier papers He93 He11 GH17, we had dealt with this problem in a less conceptual, but leaner way, sometimes with extra calculations for curve singularities.

Corollary 8.14. Consider the $\mu$-homotopy class of a reference singularity $f_{0}\left(x_{0}, \ldots, x_{n}\right)$ which satisfies assumption (8.1) and such that for any $m \geq 1$ the $\mu$-homotopy class of $f_{0}+\sum_{j=n+1}^{n+m} x_{j}^{2}$ satisfies assumption (8.2).

Fix a number $m \geq 1$. The global Torelli conjecture 8.11 (a) holds for $f_{0}$ if any only if it holds for the reference singularity $f_{0}+\sum_{j=n+1}^{n+m} x_{j}^{2}$

Proof: By 8.7), $M_{\mu}^{\text {mar }}\left(f_{0}\right)$ and $M_{\mu}^{\text {mar }}\left(f_{0}+\sum_{j=n+1}^{n+m} x_{j}^{2}\right)$ are canonically isomorphic. By theorem 7.11 (c), the classifying spaces $D_{B L}\left(f_{0}\right)$ and $D_{B L}\left(f_{0}+\sum_{j=n+1}^{n+m} x_{j}^{2}\right)$ are canonically isomorphic. It rests to see that these isomorphisms are compatible with the period maps $B L$ for $f_{0}$ and for $f_{0}+\sum_{j=n+1}^{n+m} x_{j}^{2}$. This is also rather clear from the formula (7.37) for the TEZP-structure of a suspension.

\section{Period maps AND TORElli Results FOR THE BIMOdAL SERIES AND $G_{\mathbb{Z}} \supsetneqq G^{\text {mar }}$ FOR THE SUBSERIES}

In this section we will prove for the bimodal series the strong global Torelli conjecture 8.11 (a), the conjecture 8.6 (b) - id $\notin G^{\text {smar }}$ and for the singularities with $m \wedge p$ the conjecture 8.6 (a) $G_{\mathbb{Z}}=G^{\text {mar }}$. But for the singularities in the subseries with $m \mid p$, we will see $G_{\mathbb{Z}} \supsetneqq G^{\text {mar }}$, $\left|G_{\mathbb{Z}}\right|=\infty,\left|G^{\text {mar }}\right|<\infty$. Theorem 9.1 states these results in more detail.

The singularities in the eight bimodal series $W_{1, p}^{\sharp}, S_{1, p}^{\sharp}, U_{1, p}, E_{3, p}$, $Z_{1, p}, Q_{2, p}, W_{1, p}$ and $S_{1, p}$ have as surface singularities the normal forms in table (9.1) AGV85, 15.1]. Here $p \geq 1$ and $q \geq 1$, and the parameters $\left(t_{1}, t_{2}\right)$ are in $T:=(\mathbb{C}-\{0\}) \times \mathbb{C}$.

$$
\begin{array}{ll}
W_{1,2 q-1}^{\sharp} & \left(x^{2}+y^{3}\right)^{2}+\left(t_{1}+t_{2} y\right) x y^{4+q}+z^{2} \\
W_{1,2 q}^{\sharp} & \left(x^{2}+y^{3}\right)^{2}+\left(t_{1}+t_{2} y\right) x^{2} y^{3+q}+z^{2} \\
S_{1,2 q-1}^{\sharp} & x^{2} z+y^{3} z+y z^{2}+\left(t_{1}+t_{2} y\right) x y^{3+q} \\
S_{1,2 q}^{\sharp} & x^{2} z+y^{3} z+y z^{2}+\left(t_{1}+t_{2} y\right) x^{2} y^{2+q} \\
U_{1,2 q-1} & x^{3}+x z^{2}+x y^{3}+\left(t_{1}+t_{2} y\right) y^{1+q} z^{2} \\
U_{1,2 q} & x^{3}+x z^{2}+x y^{3}+\left(t_{1}+t_{2} y\right) y^{3+q} z \\
E_{3, p} & x^{3}+x^{2} y^{3}+\left(t_{1}+t_{2} y\right) y^{9+p}+z^{2} \\
Z_{1, p} & x^{3} y+x^{2} y^{3}+\left(t_{1}+t_{2} y\right) y^{7+p}+z^{2} \\
Q_{2, p} & x^{3}+y z^{2}+x^{2} y^{2}+\left(t_{1}+t_{2} y\right) y^{6+p} \\
W_{1, p} & x^{4}+x^{2} y^{3}+\left(t_{1}+t_{2} y\right) y^{6+p}+z^{2} \\
S_{1, p} & x^{2} z+y z^{2}+x^{2} y^{2}+\left(t_{1}+t_{2} y\right) y^{5+p}
\end{array}
$$


Recall that table (5.1) lists for these singularities the Milnor number $\mu$, the characteristic polynomials $b_{j}, j \geq 1$, of the monodromy on the Orlik blocks $B_{j}$ in theorem 5.1, the order $m$ of the monodromy on $B_{1}$ and the index $r_{I}=\left[M l(f): \bigoplus_{j \geq 1} B_{j}\right]$. The order of the monodromy on $B_{2}$ is

$$
m+r_{I} \cdot p=: m_{2} .
$$

We will need the space $T^{\text {cov }}:=(\mathbb{C}-\{0\}) \times \mathbb{C}$ and the $m_{2}$-fold covering

$$
c_{T}: T^{\text {cov }} \rightarrow T, \quad\left(\tau_{1}, t_{2}\right) \mapsto\left(\tau_{1}^{m_{2}}, t_{2}\right) .
$$

For each 2-parameter family of singularities in table (9.1), we choose $f_{0}:=f_{(1,0)}$ as reference singularity. In the following, we will write $M_{\mu}^{\text {mar }},\left(M_{\mu}^{\text {mar }}\right)^{0}, G_{\mathbb{Z}}, G^{\text {mar }}, M l, H^{\infty}$ and $C^{\alpha}$ for $M_{\mu}^{\text {mar }}\left(f_{0}\right),\left(M_{\mu}^{\text {mar }}\left(f_{0}\right)\right)^{0}$, $G_{\mathbb{Z}}\left(f_{0}\right), G^{\text {mar }}\left(f_{0}\right), M l\left(f_{0}\right), H^{\infty}\left(f_{0}\right)$ and $C^{\alpha}\left(f_{0}\right)$.

We denote by $M_{T} \in G_{\mathbb{Z}}$ the monodromy of the homology bundle $\bigcup_{\left(t_{1}, t_{2}\right) \in T} \operatorname{Ml}\left(f_{\left(t_{1}, t_{2}\right)}\right) \rightarrow T$ along the cycle $\left\{\left(e^{2 \pi i s}, 0\right) \mid s \in[0,1]\right\}$. We call $M_{T}$ the transversal monodromy. By the other definition of $G^{\text {mar }}$ in [He11], $M_{T} \in G^{\text {mar }}$. As always, $\zeta:=e^{2 \pi i / m}$.

Theorem 9.1. Consider a family of bimodal series singularities in table (9.1)

(a) $M_{T}^{m_{2}}=\mathrm{id}$. Therefore the pull back to $T^{\text {cov }}$ with $c_{T}$ of the family of singularities over $T$ has trivial transversal monodromy. Thus the strong marking + id for $f_{(1,0)}$ induces a well defined strong marking for each singularity of this family over $T^{\text {cov }}$. This gives a map $T^{\text {cov }} \rightarrow\left(M_{\mu}^{\text {smar }}\right)^{0}$ and a map $T^{\operatorname{cov}} \rightarrow\left(M_{\mu}^{\text {mar }}\right)^{0}$.

(b) Both maps are isomorphisms. And - id $\notin G^{\text {smar }}$, where $G^{\text {smar }}$ is the group for the singularities of multiplicity $\geq 3$, namely the curve singularities $W_{1, p}^{\sharp}, E_{3, p}, Z_{1, p}, W_{1, p}$ and the surface singularities $S_{1, p}^{\sharp}, U_{1, p}, Q_{2, p}, S_{1, p}$. So, conjecture 8.6 (b) is true.

(c) The period map $B L: M_{\mu}^{\text {mar }} \rightarrow D_{B L}$ is an embedding. So, the strong global Torelli conjecture 8.11 (a) is true.

(d) If $m$ Xp then $G_{\mathbb{Z}}=G^{m a r}$. So, here conjecture 8.6 (a) is true.

(e) In the case of the subseries with $m \mid p, G_{\mathbb{Z}} \supsetneqq G^{\text {mar }}$. So, here conjecture 8.6 (a) is wrong. More precisely, $G^{\text {mar }}$ and $G_{\mathbb{Z}}$ are as follows. $M_{T}$ has on the 2-dimensional $\mathbb{C}$-vector space $M l_{\zeta}$ the eigenvalues 1 and $\bar{\zeta}$. Let $M l_{\zeta, 1}$ be the 1-dimensional eigenspace of $M_{T}$ on $M l_{\zeta}$ with eigenvalue 1. Then $\left|G_{\mathbb{Z}}\right|=\infty$ and $\left|G^{\text {mar }}\right|<\infty$ and

$$
G^{\text {mar }}=\left\{g \in G_{\mathbb{Z}} \mid g\left(M l_{\zeta, 1}\right)=M l_{\zeta, 1}\right\} .
$$


$\Psi\left(G_{\mathbb{Z}}\right)$ is an infinite Fuchsian group by theorem 5.1 (c). $\Psi\left(G^{\text {mar }}\right)$ is the finite subgroup of elliptic elements which fix the point $\left[M l_{\zeta, 1}\right] \in \mathcal{H}_{\zeta}$ $\left(\mathcal{H}_{\zeta}\right.$ was defined in (5.7)). And $M_{\mu}^{\text {mar }}$ consists of infinitely many copies of $T^{\mathrm{cov}}$.

Theorem 9.1 will be proved in this section in several steps. It builds on two hard results. The first and more difficult one is theorem 5.1 on $G_{\mathbb{Z}}$. The second one is easier, but still rather technical. It is the calculation of the multi-valued period map $T \rightarrow D_{B L}$. The results are fixed in theorem 9.6 .

But we prefer to present the nice geometry before the technical details. Therefore we will now explain everything what can be understood without going into the details of the Gauss-Manin connection and theorem 9.6. Afterwards we will come to the Gauss-Manin connection and theorem 9.6.

Define

$$
\alpha_{1}:=\frac{-1}{m}<\beta_{1}:=\frac{-1}{m_{2}}<0<\alpha_{2}:=\frac{1}{m_{2}}<\beta_{2}:=\frac{1}{m}
$$

and recall that $\psi_{\alpha}: H^{\infty} \rightarrow C^{\alpha}, A \mapsto e s(A, \alpha)$, is an isomorphism. Therefore and because of table (5.1)

$$
\begin{array}{r}
\operatorname{dim} C^{\beta_{1}}=\operatorname{dim} C^{\alpha_{2}}=1, \\
\operatorname{dim} C^{\alpha_{1}}=\operatorname{dim} C^{\beta_{2}}= \begin{cases}1 & \text { if } m \not p \\
2 & \text { if } m \mid p .\end{cases}
\end{array}
$$

For the cases with $m \not p$, define the 2-dimensional space

$$
\begin{aligned}
D_{B L}^{s u b}:= & \left\{\mathbb{C} \cdot\left(v_{1}+v_{2}+v_{4}\right) \mid v_{1} \in C^{\alpha_{1}}-\{0\}, v_{2} \in C^{\beta_{1}}-\{0\}, v_{4} \in C^{\beta_{2}}\right\} \\
= & \left\{\mathbb{C} \cdot\left(v_{1}^{0}+\rho_{1} v_{2}^{0}+\rho_{2} v_{4}^{0}\right) \mid\left(\rho_{1}, \rho_{2}\right) \in(\mathbb{C}-\{0\}) \times \mathbb{C}\right\} \\
& \text { for some generators } v_{1}^{0}, v_{2}^{0}, v_{4}^{0} \text { of } C^{\alpha_{1}}, C^{\beta_{1}}, C^{\beta_{2}} \\
\cong & (\mathbb{C}-\{0\}) \times \mathbb{C} .
\end{aligned}
$$

For the cases with $m \mid p$, the polarizing form $S$ defines an indefinite hermitian form $((a, b) \mapsto S(a, \bar{b}))$ on $H_{\zeta}^{\infty}$. This follows from the corresponding statement for $h_{\zeta}$ on $M l_{\zeta}$ in theorem 5.1, from lemma 2.2 (b) and from the relation between Seifert form $L$ and polarizing form $S$, see 4.20). Thus we get a half-plane

$$
\begin{aligned}
\mathcal{H}\left(C^{\alpha_{1}}\right) & :=\left\{\mathbb{C} \cdot v \mid v \in C^{\alpha_{1}} \text { with } S\left(\psi_{\alpha_{1}}^{-1}(v), \overline{\psi_{\alpha_{1}}^{-1}(v)}\right)<0\right\} \\
& \subset \mathbb{P}\left(C^{\alpha}\right) .
\end{aligned}
$$


Now define for the cases with $m \mid p$ the 3-dimensional space

$$
\begin{aligned}
D_{B L}^{\text {sub }} & :=\left\{\mathbb{C} \cdot\left(v_{1}+v_{2}+v_{4}\right) \mid v_{1} \in C^{\alpha_{1}}-\{0\} \text { with }\left[\mathbb{C} \cdot v_{1}\right] \in \mathcal{H}\left(C^{\alpha_{1}}\right),\right. \\
& \left.v_{2} \in C^{\beta_{1}}-\{0\}, v_{4} \in \mathbb{C} \cdot \psi_{\beta_{2}}\left(\overline{\psi_{\alpha_{1}}^{-1}\left(v_{1}\right)}\right) \subset C^{\beta_{2}}\right\} \\
\cong & \mathcal{H}\left(C^{\alpha_{1}}\right) \times(\mathbb{C}-\{0\}) \times \mathbb{C} .
\end{aligned}
$$

Theorem 9.2. (a) $D_{B L}^{s u b}$ embeds canonically into $D_{B L}$.

(b) For suitable $v_{1}^{0} \in C^{\alpha_{1}}-\{0\}, v_{2}^{0} \in C^{\beta_{1}}-\{0\}$ and for $v_{4}^{0}:=$ $\psi_{\beta_{2}}\left(\overline{\psi_{\alpha_{1}}^{-1}\left(v_{1}^{0}\right)}\right) \in C^{\beta_{2}}-\{0\}$, the multi-valued period map $B L_{T}: T \rightarrow D_{B L}$ has its image in $D_{B L}^{s u b}$ and takes the form

$$
\left(t_{1}, t_{2}\right) \mapsto \mathbb{C} \cdot\left(v_{1}^{0}+t_{1}^{1 / m_{2}} \cdot v_{2}^{0}+\left(\frac{t_{2}}{t_{1}}+r\left(t_{1}\right)\right) v_{4}^{0}\right)
$$

with

$$
r\left(t_{1}\right)= \begin{cases}0 & \text { in the cases }\left(r_{I}=1 \& p \geq 3\right), \\ & \text { the cases }\left(r_{I}=2 \& p \geq 2\right) \\ & \text { and the case } U_{1,2}, \\ c_{T} \cdot t_{1} & \text { in the cases }\left(r_{I}=2 \& p=1\right) \\ & \text { and the cases } W_{1,2}^{\sharp} \text { and } S_{1,2}^{\sharp}, \\ c_{T} \cdot t_{1}^{2} & \text { in the cases }\left(r_{I}=1 \& p=1\right),\end{cases}
$$

for a suitable constant $c_{T} \in \mathbb{C}$. In the cases with $m \mid p$, the transversal monodromy $M_{T}$ has on $C^{\alpha_{1}}$ the eigenvalues 1 and $\bar{\zeta}$, and $\mathbb{C} \cdot v_{1}^{0}$ is the eigenspace with eigenvalue 1 . The class $\left[\mathbb{C} \cdot v_{1}^{0}\right]$ is in $\mathcal{H}\left(C^{\alpha_{1}}\right)$.

(c) The induced period map $B L_{T^{c o v}}: T^{c o v} \rightarrow D_{B L}^{s u b}$ is an isomorphism if $m$ Xp and an isomorphism to the fiber above $\left[\mathbb{C} \cdot v_{1}^{0}\right] \in \mathcal{H}\left(C^{\alpha_{1}}\right)$ of the projection $D_{B L}^{s u b} \rightarrow \mathcal{H}\left(C^{\alpha_{1}}\right)$ if $m \mid p$.

(d) In the case of the subseries $U_{1,9 r}, G^{\text {mar }}$ contains an element $g_{3}$ such that $\Psi\left(g_{3}\right)$ is elliptic of order 18 (for all subseries with $p=m \cdot r$, $\Psi\left(M_{T}\right)$ is elliptic of order $m$, for $\left.U_{1,9 r} m=9\right)$.

(e) $f_{\left(t_{1}, t_{2}\right)}$ and $f_{\left(\widetilde{t}_{1}, \widetilde{t}_{2}\right)}$ are right equivalent

$$
\Longleftrightarrow\left\{\begin{array}{l}
\exists k \in \mathbb{Z} \text { with }\left(\widetilde{t}_{1}, \widetilde{t}_{2}\right)=\left(\zeta^{r_{I} p k} \cdot t_{1}, \zeta^{\left(r_{I} p+2\right) k} \cdot t_{2}\right) \\
\quad \text { for all } 8 \text { series except } U_{1,2 q}, \\
\exists k \in \mathbb{Z} \text { and } \varepsilon \in\{ \pm 1\} \text { with } \\
\quad\left(\widetilde{t_{1}}, \widetilde{t}_{2}\right)=\left(\varepsilon \zeta^{r_{I} p k} \cdot t_{1}, \varepsilon \zeta^{\left(r_{I} p+2\right) k} \cdot t_{2}\right) \text { for } U_{1,2 q}
\end{array}\right.
$$

The parts (a), (b) and (d) of theorem 9.2 will be proved after theorem 9.6 .

Proof of theorem 9.2 (c) and (e):

(c) This follows immediately from 9.10). 
(e) First we prove $\Leftarrow$. We give explicit coordinate changes. A case by case comparison with the normal forms in table (9.1) shows that the following equality (9.13) holds. Here $\left(\delta_{1}, \delta_{2}, \delta_{3}\right)$ are as in table (9.14), and $k \in \mathbb{Z}$.

$$
\begin{aligned}
& f_{\left(t_{1}, t_{2}\right)}\left(x \cdot \zeta^{\delta_{1} \cdot k}, y \cdot \zeta^{\delta_{2} \cdot k}, z \cdot \zeta^{\delta_{3} \cdot k}\right)=f_{\left(t_{1} \cdot \zeta^{r} I^{p k}, t_{2} \cdot \zeta^{\left(r_{I} p+2\right) k}\right)}(x, y, z) . \\
& \begin{array}{lll}
\delta_{1} & \delta_{2} & \delta_{3}
\end{array} \\
& W_{1, p}^{\sharp} \text { and } W_{1, p} \quad 3 \quad 2 \quad 0 \\
& S_{1, p}^{\sharp} \text { and } S_{1, p} \quad 3 \quad 2 \quad 4 \\
& \begin{array}{llll}
U_{1, p} & 3 & 2 & 3
\end{array} \\
& \begin{array}{llll}
E_{3, p} & 6 & 2 & 0
\end{array} \\
& \begin{array}{llll}
Z_{1,0} & 4 & 2 & 0
\end{array} \\
& \begin{array}{llll}
Q_{2, p} & 4 & 2 & 5
\end{array}
\end{aligned}
$$

In the case $U_{1,2 q}$ we have additionally

$$
f_{\left(t_{1}, t_{2}\right)}(x, y,-z)=f_{\left(-t_{1},-t_{2}\right)}(x, y, z) .
$$

This shows $\Leftarrow$.

Now we prove $\Rightarrow$. Let $f_{\left(t_{1}, t_{2}\right)}$ and $f_{\left(\widetilde{t}_{1}, \widetilde{t}_{2}\right)}$ be right equivalent. Then $B L_{T}\left(t_{1}, t_{2}\right)$ and $B L_{T}\left(\widetilde{t}_{1}, \widetilde{t}_{2}\right)$ are isomorphic, so a $g \in G_{\mathbb{Z}}$ with $g\left(B L_{T}\left(t_{1}, t_{2}\right)\right)=B L_{T}\left(\widetilde{t}_{1}, \widetilde{t}_{2}\right)$ exists. We claim that $v_{1}^{0}, v_{2}^{0}$ and $v_{4}^{0}$ are eigenvectors of $g$ with some eigenvalues $\lambda_{1}, \lambda_{2}$ and $\overline{\lambda_{1}}$. For $v_{2}^{0}$ this is trivial as $\operatorname{dim} C^{\beta_{1}}=1$, for $v_{1}^{0}$ in the case $m \not p$ also. In the case $m \mid p$, it follows for $v_{1}^{0}$ from 9.10 . For $v_{4}^{0}$ use $v_{4}^{0}=\psi_{\beta_{2}}\left(\overline{\psi_{\alpha_{1}}^{-1}\left(v_{1}^{0}\right)}\right)$. We claim also

$$
\lambda_{1} \in \operatorname{Eiw}(\zeta), \quad \lambda_{2} \in \operatorname{Eiw}\left(e^{2 \pi i / m_{2}}\right) .
$$

For $\lambda_{2}$ this is a consequence of the following three facts and of theorem 2.9 (a)\&(b).

(i) The 1-dimensional eigenspace $M l_{e^{2 \pi i / m_{2}}}$ is already defined over $\mathbb{Q}\left(e^{2 \pi i / m_{2}}\right)$. Therefore $\lambda_{2} \in \mathbb{Q}\left(e^{2 \pi i / m_{2}}\right)$.

(ii) $\left|\lambda_{2}\right|=1$ because $L$ pairs $M l_{e^{2 \pi i / m_{2}}}$ and $M l_{e^{-2 \pi i / m_{2}}}$.

(iii) $\lambda_{2}$ is an algebraic integer because $g \in G_{\mathbb{Z}}$.

If $m \not p$, the same reasoning applies also to $\lambda_{1}$. Suppose for a moment $m \mid p$.

By part (b), the transversal monodromy $M_{T}$ acts on $C^{\alpha_{1}}$ and on $H_{\zeta}^{\infty}$ with eigenvalues 1 and $\zeta$, and the 1 -dimensional eigenspaces with eigenvalue 1 are $\mathbb{C} \cdot v_{1}^{0}$ and $\mathbb{C} \cdot \psi_{\alpha_{1}}^{-1}\left(v_{1}^{0}\right)$. Therefore $\mathbb{C} \cdot \psi_{\alpha_{1}}^{-1}\left(v_{1}^{0}\right)$ is already defined over $\mathbb{Q}(\zeta)$, i.e. $\mathbb{C} \cdot \psi_{\alpha_{1}}^{-1}\left(v_{1}^{0}\right) \cap H_{\mathbb{Q}(\zeta)}^{\infty}$ is a 1-dimensional $\mathbb{Q}(\zeta)$ vector space. This implies (i) $\lambda_{1} \in \mathbb{Q}(\zeta)$. (ii) $\left|\lambda_{1}\right|=1$ holds because $v_{1}^{0} \in \mathcal{H}\left(C^{\alpha_{1}}\right)$. And (iii) ( $\lambda_{1}$ is an algebraic integer) holds anyway. 
Again with theorem 2.9 (a)\&(b) we conclude $\lambda_{1} \in \operatorname{Eiw}(\zeta)$. Now 9.16 is proved in all cases.

The equality $g\left(B L_{T}\left(t_{1}, t_{2}\right)\right)=B L_{T}\left(\widetilde{t}_{1}, \widetilde{t}_{2}\right)$ becomes

$$
\begin{aligned}
& \mathbb{C} \cdot\left(\lambda_{1} \cdot v_{1}^{0}+\lambda_{2} \cdot t_{1}^{1 / m_{2}} \cdot v_{2}^{0}+\overline{\lambda_{1}}\left(\frac{t_{2}}{t_{1}}+r\left(t_{1}\right)\right) \cdot v_{4}^{0}\right) \\
= & \mathbb{C} \cdot\left(v_{1}^{0}+\widetilde{t}_{1}^{1 / m_{2}} \cdot v_{2}^{0}+\left(\frac{\widetilde{t}_{2}}{\widetilde{t}_{1}}+r\left(\widetilde{t_{1}}\right)\right) \cdot v_{4}^{0}\right), \\
\text { so } \quad & \widetilde{t}_{1}^{1 / m_{2}}=\lambda_{2}{\overline{\lambda_{1}}}^{\prime} \cdot t_{1}^{1 / m_{2}}, \quad \frac{\widetilde{t}_{2}}{\widetilde{t}_{1}}+r\left(\widetilde{t_{1}}\right)={\overline{\lambda_{1}}}^{2}\left(\frac{t_{2}}{t_{1}}+r\left(t_{1}\right)\right), \\
\text { so } \quad & \widetilde{t}_{1}=\lambda_{2}^{m_{2}}{\overline{\lambda_{1}}}^{m_{2}} \cdot t_{1}, \\
\text { and } \quad & \widetilde{t}_{2}={\overline{\lambda_{1}}}^{2} \cdot \frac{\widetilde{t}_{1}}{t_{1}} \cdot t_{2}+\widetilde{t}_{1} \cdot\left({\overline{\lambda_{1}}}^{2} \cdot r\left(t_{1}\right)-r\left(\widetilde{t_{1}}\right)\right) .
\end{aligned}
$$

Because of (9.16), we can write $\lambda_{1}$ and $\lambda_{2}$ as follows, here $k, l \in \mathbb{Z}$ and $\varepsilon_{1}, \varepsilon_{2} \in\{ \pm 1\}$.

$$
\begin{array}{lll} 
& \lambda_{1} & \lambda_{2} \\
\text { All cases with } m \equiv 0(2), m_{2} \equiv 0(2) & \bar{\zeta}^{k} & e^{2 \pi i l / m_{2}} \\
\text { The cases } W_{1,2 q-1}^{\sharp} \text { and } S_{1,2 q-1}^{\sharp} & \varepsilon_{2} \cdot \bar{\zeta}^{k} & \varepsilon_{2} \cdot e^{2 \pi i l / m_{2}} \\
\text { The cases } U_{1,2 q-1} & \varepsilon_{1} \cdot \bar{\zeta}^{k} & e^{2 \pi i l / m_{2}} \\
\text { The cases } U_{1,2 q} & \varepsilon_{1} \cdot \bar{\zeta}^{k} & \varepsilon_{2} \cdot e^{2 \pi i l / m_{2}}
\end{array}
$$

One checks that 9.17 boils down to

$$
\widetilde{t}_{1}=\zeta^{r_{I} p k} \cdot t_{1}, \quad \widetilde{t}_{2}=\zeta^{\left(r_{I} p+2\right) k} \cdot t_{2},
$$

in all cases except $U_{1,2 q}$. In the cases $U_{1,2 q}$, it boils down to

$$
\widetilde{t}_{1}=\varepsilon_{1} \varepsilon_{2} \cdot \zeta^{p k} \cdot t_{1}, \quad \widetilde{t}_{2}=\varepsilon_{1} \varepsilon_{2} \cdot \zeta^{(p+2) k} .
$$

This finishes the proof of $\Rightarrow$ and the proof of theorem 9.2 (e).

The statements in theorem 9.1 on the transversal monodromy $\left(M_{T}^{m_{2}}=\mathrm{id}, M_{T}\right.$ has the eigenvalues 1 and $\zeta$ on $\left.M l_{\zeta}\right)$ will be proved after theorem 9.6. The rest of theorem 9.1 will be proved now.

Proof of theorem 9.1 (without the statements on $M_{T}$ ):
(a) This is clear.
(b) Consider the maps 


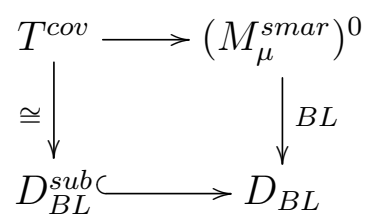

As $T^{\text {cov }} \hookrightarrow D_{B L}^{s u b} \hookrightarrow D_{B L}$ is an embedding, $T^{\text {cov }} \rightarrow\left(M_{\mu}^{\text {smar }}\right)^{0}$ is an embedding.

Both spaces $T^{\text {cov }}$ and $\left(M_{\mu}^{\text {smar }}\right)^{0}$ are locally $\mu$-constant strata of universal unfoldings and are therefore smooth of dimension $2 . D_{B L}^{s u b}$ is almost closed in $D_{B L}$. Its closure consists of itself and the space $\left\{\mathbb{C} \cdot\left(v_{1}+v_{4}\right) \mid v_{1}\right.$ and $v_{4}$ as in (9.7) or $\left.(9.9)\right\}$ (so $\left.v_{2}=0\right)$. No $g \in G_{\mathbb{Z}}$ maps a point of this space to a point of $D_{B L}^{s u b}$. And $T^{\text {cov }}$ contains representatives of any right equivalence class in the $\mu$-homotopy family. Therefore the image of $\left(M_{\mu}^{s m a r}\right)^{0}$ in $D_{B L}$ cannot be bigger than $D_{B L}^{s u b}$. Thus $T^{\text {cov }} \cong\left(M_{\mu}^{\text {smar }}\right)^{0}$.

In the case of singularities of multiplicity $2, M_{\mu}^{s m a r} \cong M_{\mu}^{\text {mar }}$ holds anyway by theorem 8.5 (c), and then also $\left(M_{\mu}^{\text {smar }}\right)^{0} \cong\left(M_{\mu}^{\text {mar }}\right)^{0}$ holds.

Consider the case of singularities of multiplicity $\geq 3$. Then $-\mathrm{id} \in$ $G_{\mathbb{Z}}$ acts nontrivially on $M_{\mu}^{s m a r}$ by theorem 8.5 (c). It acts trivially on $D_{B L}$. The map $\left(M_{\mu}^{s m a r}\right)^{0} \rightarrow D_{B L}$ is an embedding. Therefore - id $\in G_{\mathbb{Z}}$ does not act on $\left(M_{\mu}^{s m a r}\right)^{0}$, therefore - id $\notin G^{\text {smar }}$. Then $\left(M_{\mu}^{\text {smar }}\right)^{0} \rightarrow\left(M_{\mu}^{\text {mar }}\right)^{0}$ is an isomorphism by theorem 8.5 (c).

(c) for $m / p$ and $(\mathrm{d})$ : $\left(M_{\mu}^{\text {mar }}\right)^{0} \stackrel{\cong}{\longrightarrow} T^{\text {cov }} \stackrel{\cong}{\longrightarrow} D_{B L}^{\text {sub }} \hookrightarrow D_{B L}$ is an embedding. $G_{\mathbb{Z}}=G^{\text {mar }}$ would imply $M_{\mu}^{\text {mar }}=\left(M_{\mu}^{\text {mar }}\right)^{0}$. Therefore it is sufficient to prove $G_{\mathbb{Z}}=G^{\text {mar }}$.

Let $g_{1} \in G_{\mathbb{Z}}$. It acts on $D_{B L}^{s u b}$. By the proof of theorem 9.2 (e), the map

$$
\left(M_{\mu}^{\text {mar }}\right)^{0} / G^{\text {mar }} \rightarrow D_{B L}^{s u b} / G_{\mathbb{Z}}
$$

is an isomorphism. Therefore an element $g_{2} \in G^{\text {mar }}$ exists which acts in the same way on $D_{B L}^{s u b}$ as $g_{1}$. Consider $g_{3}:=g_{1} \circ g_{2}^{-1}$. It acts trivially on $D_{B L}^{s u b}$. It has eigenvalues $\lambda_{1}, \lambda_{2}$ and $\overline{\lambda_{1}}$ on $C^{\alpha_{1}}, C^{\beta_{1}}$ and $C^{\beta_{2}}$. Therefore

$$
\begin{aligned}
& \mathbb{C}\left(v_{1}+v_{2}+v_{4}\right)=\mathbb{C}\left(\lambda_{1} \cdot v_{1}+\lambda_{2} \cdot v_{2}+\overline{\lambda_{1}} \cdot v_{4}\right) \\
& \text { for any } \mathbb{C}\left(v_{1}+v_{2}+v_{4}\right) \in D_{B L}^{s u b}, \\
& \text { thus } \lambda_{2} \overline{\lambda_{1}}=1, \quad{\overline{\lambda_{1}}}^{2}=\mathrm{id}, \quad \text { so } \lambda_{1}=\lambda_{2} \in\{ \pm 1\}, \\
& \text { and } g_{3}=\lambda_{1} \cdot \text { id on } M l_{\zeta} \oplus M l_{e^{2 \pi i / m_{2}}} .
\end{aligned}
$$

$G_{\mathbb{Z}}$ was determined in theorem 5.1 (b). It contains very few automorphisms $g_{3}$ with (9.23). Formula (5.6) and table (5.1) show that the 
$\operatorname{group}\left\{g \in G_{\mathbb{Z}} \mid g= \pm \mathrm{id}\right.$ on $\left.M l_{\zeta} \oplus M l_{e^{2 \pi i / m_{2}}}\right\}$ is as follows:

$$
\begin{aligned}
& \{ \pm \mathrm{id}\} \quad \text { in the cases } W_{1,2 q-1}^{\sharp}, S_{1,2 q-1}^{\sharp}, U_{1,2 q}, E_{3, p}, Z_{1, p}, \\
& \left\{ \pm \mathrm{id}, \pm\left(\left.\mathrm{id}\right|_{B_{1}} \times\left.\left(-M_{h}^{m_{2} / 2}\right)\right|_{B_{2}}\right)\right\} \quad \text { in the cases } W_{1,2 q}^{\sharp}, S_{1,2 q}^{\sharp}, U_{1,2 q-1}, \\
& \left\{ \pm \mathrm{id}, \pm\left(\left.\left(-M_{h}^{m / 2}\right)\right|_{B_{1}} \times\left.\mathrm{id}\right|_{B_{2}}\right)\right\} \quad \text { in the cases } Q_{2, p}, W_{1, p}, S_{1, p} .
\end{aligned}
$$

\section{Claim:}

$$
\left\{g \in G_{\mathbb{Z}} \mid g= \pm \text { id on } M l_{\zeta} \oplus M l_{e^{2 \pi i / m_{2}}}\right\}=G_{\mathcal{R}}^{\text {mar }} .
$$

This claim shows $g_{3} \in G_{\mathcal{R}}^{\text {mar }}$ and $g_{1} \in G^{\text {mar }}$, so that $G_{\mathbb{Z}}=G^{\text {mar }}$.

The inclusion $\supset$ in $(9.25)$ holds because of the following: Any element of $G_{\mathcal{R}}^{\text {mar }}=G_{\mathcal{R}}^{\text {mar }}\left(f_{(1,0)}\right)$ acts on $D_{B L}^{\text {sub }}$ with $B L_{T}(1,0)$ as fixed point. The proof of theorem 9.2 (e) shows that it acts then trivially on $D_{B L}^{s u b}$.

The group $G_{\mathcal{R}}^{\text {mar }}$ contains $\pm \mathrm{id}$. In order to prove equality in 9.25 for the cases in the second and third line of (9.24), it is sufficient to show that $G_{\mathcal{R}}^{\text {mar }}$ contains more elements than \pm id. Equivalent is that $G_{\mathcal{R}}^{\text {smar }}(f)$ for a generic singularity $f$ with multiplicity $\geq 3$ contains one other element than $+\mathrm{id}$. The following table lists coordinate changes which give such an element.

$$
\begin{array}{ll}
W_{1,2 q}^{\sharp} & (x, y) \mapsto(-x, y) \\
S_{1,2 q}^{\sharp} & (x, y, z) \mapsto(-x, y, z) \\
U_{1,2 q-1} & (x, y, z) \mapsto(x, y,-z) \\
Q_{2, p} & (x, y, z) \mapsto(x, y,-z) \\
W_{1, p} & (x, y) \mapsto(-x, y) \\
S_{1, p} & (x, y, z) \mapsto(-x, y, z)
\end{array}
$$

This proves the claim and finishes the proof of (c) for $m \not p$ and (d).

(c) for $m \mid p$ and (e): First we prove (9.4).

$\Psi\left(M_{T}\right)$ is an elliptic element with fixed point $\left[M l_{\zeta, 1}\right] \in \mathcal{H}_{\zeta}$ and angle $\frac{2 \pi}{m}=\arg \left(\frac{\zeta}{1}\right)$. All elements of $G^{\text {mar }}$, including $M_{T}$, act on $\mathcal{H}\left(C^{\alpha_{1}}\right)$ as elliptic elements with fixed point $\left[\mathbb{C} \cdot v_{1}^{0}\right]$, because all elements in $G^{\text {mar }}$ act on $\left(M_{\mu}^{\text {mar }}\right)^{0}$ and on its image $B L_{T^{c o v}}\left(\left(M_{\mu}^{\text {mar }}\right)^{0}\right) \subset D_{B L}^{s u b}$. Therefore all elements of $G^{\text {mar }}$ act on $\mathcal{H}_{\zeta}$ as elliptic elements with fixed point $\left[M l_{\zeta, 1}\right]$. This shows $\subset$ in 9.4 .

Now let $g_{1} \in\left\{g \in G_{\mathbb{Z}} \mid g\left(M l_{\zeta, 1}\right)=M l_{\zeta, 1}\right\}$. It has an eigenvalue $\lambda_{1}$ on $M l_{\zeta, 1}$ and an eigenvalue $\lambda_{2}$ on the other eigenspace within $M l_{\zeta}$ (which is the $h_{\zeta^{-}}$orthogonal subspace of $M l_{\zeta}$ ). By (9.16) $\lambda_{1}$ and $\lambda_{2} \in \operatorname{Eiw}(\zeta)$. Therefore $\Psi\left(g_{1}\right)$ is an elliptic element with fixed point $\left[M l_{\zeta, 1}\right] \in \mathcal{H}_{\zeta}$ and angle $\arg \frac{\lambda_{2}}{\lambda_{1}}$. 
In all cases except possibly $U_{1,9 r}$, the product $g_{2}=g_{1} \circ M_{T}^{k}$ for a suitable $k \in \mathbb{Z}$ acts trivially on $\mathcal{H}_{\zeta}$. In the cases $U_{1,9 r}$, the product $g_{2}=g_{1} \circ g_{3}^{k}$ for $g_{3} \in G^{\text {mar }}$ as in theorem 9.2 (d) does the same.

Formula (5.9) in theorem 5.1 (c) applies to $g_{2}$ and shows $g_{2} \in$ $\left\{ \pm M_{h}^{k} \mid k \in \mathbb{Z}\right\}$. Therefore $g_{2} \in G^{\text {mar }}$ and $g_{1} \in G^{\text {mar }}$. This shows $\supset$ in $(9.4)$, so $(9.4)$ is now proved.

Especially, $\Psi\left(G^{\text {mar }}\right)$ and $G^{\text {mar }}$ are finite. By theorem 5.1 (c), $\Psi\left(G_{\mathbb{Z}}\right)$ and $G_{\mathbb{Z}}$ are infinite. Therefore $G_{\mathbb{Z}} \supsetneqq G^{\text {mar }}$.

By theorem 8.5 (a), $M_{\mu}^{\text {mar }}$ consists of infinitely many copies of $\left(M_{\mu}^{\text {mar }}\right)^{0}$.

If two different copies would have intersecting images in $D_{B L}$ under the period map $B L$, the images would coincide, and there would be a copy different from $\left(M_{\mu}^{\text {mar }}\right)^{0}$ with the same image in $D_{B L}$ as $\left(M_{\mu}^{\text {mar }}\right)^{0}$. An element $g_{3} \in G_{\mathbb{Z}}$ which maps $\left(M_{\mu}^{\text {mar }}\right)^{0}$ to this copy would be in $\left\{g \in G_{\mathbb{Z}} \mid g\left(M l_{\zeta, 1}\right)=M l_{\zeta, 1}\right\}-G^{\text {mar }}=\emptyset$, a contradiction. Therefore $B L: M_{\mu}^{\text {mar }} \rightarrow D_{B L}^{s u b}$ is an embedding.

Remarks 9.3. (i) The arithmetic triangle group of type $(2,3,14)$ for $Z_{1,0}$ in theorem 3.6 contains elliptic elements of order 3 although $\arg \zeta=$ $\frac{2 \pi}{14}$ and the matrices defining these elliptic elements are in $G L(2, \mathbb{Z}[\zeta])$. The eigenspaces in $M(2 \times 1, \mathbb{C})$ of these matrices are not defined over $\mathbb{Q}(\zeta)$, but only over $\mathbb{Q}\left(e^{2 \pi i / 3}, \zeta\right)$. This example shows that $(9.16)$ in the case $m \mid p$ and the arguments proving it are nontrivial.

(ii) In 1993, the second author worked on the Torelli conjecture for the unmarked bimodal series singularities. He missed to consider $M_{T}$ carefully and thus was not sure which elliptic elements fix $\left[\mathbb{C} \cdot v_{1}^{0}\right] \in$ $\mathcal{H}\left(C^{\alpha_{1}}\right)$. Therefore he could not prove the Torelli conjecture for the unmarked singularities in the subseries $S_{1,10 r}^{\sharp}, S_{1,10 r}$ and $Z_{1,14 r}$. Now theorem 9.1 gives the marked and unmarked Torelli theorem for all bimodal series singularities.

Now we come to the spectral numbers and the classifying space $D_{B L}$.

Lemma 9.4. Consider a family of bimodal series singulariteis in table (9.1).

(a) The spectral numbers $\alpha_{1}, \ldots, \alpha_{\mu}$ with $\alpha_{1} \leq \ldots \leq \alpha_{\mu}$ satisfy

$$
\begin{array}{r}
\alpha_{1}=\frac{-1}{m}<\alpha_{2}=\frac{1}{m_{2}}<\alpha_{3} \leq \ldots \leq \alpha_{\mu-2} \\
<\alpha_{\mu-1}=1-\frac{1}{m_{2}}<\alpha_{\mu}=1+\frac{1}{m}
\end{array}
$$

and are uniquely determined by this and the characteristic polynomial $\prod_{j \geq 1} b_{j}$ of the monodromy with $b_{j}$ as in table (5.1. 
(b) Recall from (9.5) $\beta_{1}=\frac{-1}{m_{2}}=-\alpha_{2}$ and $\beta_{2}=\frac{1}{m}=-\alpha_{1}$. Then

$$
\operatorname{dim} C^{\alpha_{1}}= \begin{cases}1 & \text { if } m \not p \\ 2 & \text { if } m \mid p\end{cases}
$$

$\operatorname{dim} C^{\beta}= \begin{cases}1 \quad \text { for } \beta \in\left(\alpha_{1}, \beta_{2}\right) \cap \frac{1}{m_{2}}(\mathbb{Z}-\{0\}) \text { if } r_{I}=1, \\ \text { and for } \beta \in\left(\alpha_{1}, \beta_{2}\right) \cap\left(\frac{1}{m_{2}}+\frac{2}{m_{2}} \mathbb{Z}\right) \text { if } r_{I}=2, \\ 0 \quad \text { for other } \beta \in\left(\alpha_{1}, \beta_{2}\right) .\end{cases}$

The following two pictures illustrate this for $2 m<p<3 m$, the first for $r_{I}=1$, the second for $r_{I}=2$.
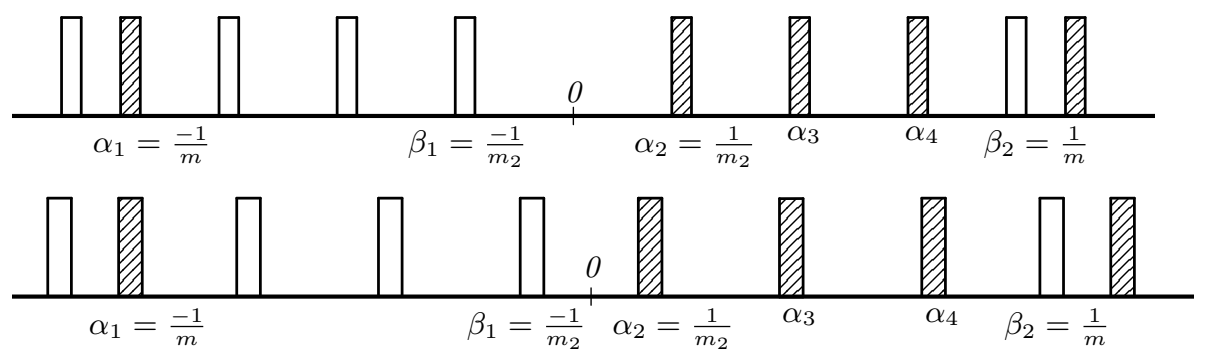

(c) Denote by $(*)$ the condition

$$
\left.(*): \quad \beta \in\left(\alpha_{1}, 0\right) \text { with } C^{\beta} \neq\{0\} \quad \text { (then } \operatorname{dim} C^{\beta}=1\right) \text {. }
$$

If $m$ Xp the classfying space $D_{B L}$ in [He99] is

$$
\begin{aligned}
D_{B L}= & \left\{\mathbb{C} \cdot\left(v_{1}+\sum_{\beta:(*)} v_{(\beta)}+v_{2}\right) \mid\right. \\
& \left.v_{1} \in C^{\alpha_{1}}-\{0\}, v_{(\beta)} \in C^{\beta}, v_{2} \in C^{\beta_{2}}\right\} \\
\cong & \mathbb{C}^{N_{B L}} \quad \text { with } N_{B L}:=|\{\beta:(*)\}|+1 .
\end{aligned}
$$

In (9.8) $\mathcal{H}\left(C^{\alpha_{1}}\right)$ was defined for $m \mid p$. If $m \mid p$ then $D_{B L}$ is

$$
\begin{aligned}
D_{B L}= & \left\{\mathbb{C} \cdot\left(v_{1}+\sum_{\beta:(*)} v_{(\beta)}+v_{2}\right) \mid\right. \\
& v_{1} \in C^{\alpha_{1}}-\{0\} \text { with }\left[\mathbb{C} \cdot v_{1}\right] \in \mathcal{H}\left(C^{\alpha_{1}}\right) \\
& \left.v_{(\beta)} \in C^{\beta}, v_{2} \in \mathbb{C} \cdot \psi_{\beta_{2}}\left(\overline{\psi_{\alpha_{1}}^{-1}\left(v_{1}\right)}\right) \subset C^{\beta_{2}}\right\} \\
\cong & \mathcal{H}\left(C^{\alpha_{1}}\right) \times \mathbb{C}^{N_{B L}} \quad \text { with } N_{B L}:=|\{\beta:(*)\}|+1 .
\end{aligned}
$$

Proof: (a) The spectral numbers are well known AGV88, 13.3.4, p. 389]. They also follow from corollary 7.17 and the proof of theorem 9.6.

(b) 9.28) follows from $\operatorname{dim} C^{\alpha_{1}}=\operatorname{dim} M l_{\zeta}$ and $\Phi_{m} \backslash b_{2} \Longleftrightarrow m \not p$. 9.29 follows from the values of $b_{j}$ in table (5.1). 
(c) The spectral numbers and the numbers $\beta$ with $C^{\beta} \neq\{0\}$ give for each $\mathcal{L}_{0} \in D_{B L}$

$$
\mathcal{L}_{0}=\mathbb{C} \cdot \sigma_{1} \oplus \mathcal{L}_{0} \cap \bigoplus_{\beta: \alpha_{2} \leq \beta \leq \beta_{2}} C^{\beta} \oplus V^{>\beta_{2}}
$$

where

$$
\alpha\left(\sigma_{1}\right)=\alpha_{1}, \quad \sigma_{1} \in C^{\alpha_{1}} \oplus \bigoplus_{\beta:(*)} C^{\beta} \oplus C^{\beta_{2}} .
$$

Here observe that for $\beta$ with $\alpha_{2} \leq \beta<\beta_{2}$ and $C^{\beta} \neq\{0\}$, the space $C^{\beta}$ is one-dimensional and is generated by the principal part of a section in $\mathcal{L}_{0}$.

If $m \wedge p$ then $\operatorname{dim} C^{\beta_{2}}=1$ and $C^{\beta_{2}}$ is not generated by the principal part of a section in $\mathcal{L}_{0}$. If $m \mid p$ then $\operatorname{dim} C^{\beta_{2}}=2$ and the onedimensional subspace $\left\{v \in C^{\beta_{2}} \mid K_{f}^{(-2)}\left(v, s\left(\sigma_{1}, \alpha_{1}\right)\right)=0\right\} \subset C^{\beta_{2}}$ is in $\mathcal{L}_{0}$, because then $\beta_{2}$ is a spectral number with multiplicity 1 . And then the principal part $s\left(\sigma_{1}, \alpha_{1}\right)$ must be compatible with a polarized Hodge structure of weight 2 on $H_{\zeta}^{\infty} \oplus H_{\bar{\zeta}}^{\infty}$. This amounts to $\left[\mathbb{C} \cdot s\left(\sigma_{1}, \alpha_{1}\right)\right] \in \mathcal{H}\left(C^{\alpha_{1}}\right)$. Especially then

$$
C^{\beta_{2}}=\mathbb{C} \cdot \psi_{\beta_{2}}\left(\overline{\psi_{\alpha_{1}}^{-1} s\left(\sigma_{1}, \alpha_{1}\right)}\right) \oplus\left\{v \in C^{\beta_{2}} \mid K_{f}^{(-2)}\left(v, s\left(\sigma_{1}, \alpha_{1}\right)\right)=0\right\}(, 9.34)
$$

and $\sigma_{1}$ can be chosen with

$$
\alpha\left(\sigma_{1}\right)=\alpha_{1}, \quad \sigma_{1} \in C^{\alpha_{1}} \oplus \bigoplus_{\beta:(*)} C^{\beta} \oplus \mathbb{C} \cdot \psi_{\beta_{2}}\left(\overline{\psi_{\alpha_{1}}^{-1} s\left(\sigma_{1}, \alpha_{1}\right)}\right) .
$$

$\sigma_{1}$ is (up to rescaling) uniquely determined by $(9.33)$ if $m \not p$ and by (9.35) if $m \mid p$. And it can be chosen freely with (9.33) respectively with (9.35) and $\left[\mathbb{C} \cdot s\left(\sigma_{1}, \alpha_{1}\right)\right] \in \mathcal{H}\left(C^{\alpha_{1}}\right)$. The condition $(\delta) K_{f}^{(-2)}\left(\mathcal{L}_{0}, \mathcal{L}_{0}\right)=0$ on $D_{B L}$ directly before theorem 7.11 implies that $\mathcal{L}_{0} \cap \bigoplus_{\alpha_{2} \leq \beta \leq \beta_{2}} C^{\beta}$ is uniquely determined by $\sigma_{1}$. Therefore $\mathcal{L}_{0}$ is uniquely determined by $\sigma_{1}$. Therefore $D_{B L}$ is as stated in 9.30 and 9.31 .

Remarks 9.5. (i) All the normal forms in table (9.1) except $W_{1, p}^{\sharp}$ are Newton nondegenerate. But also the normal form $f_{p}(x, y, \widetilde{z})$ for $W_{1, p}^{\sharp}$ in table (9.1) can be made easily Newton nondegenerate with the coordinate change $\widetilde{z}=z+i\left(x^{2}+y^{3}\right)$. Then

$$
\begin{aligned}
f_{p}\left(x, y, z+i\left(x^{2}+y^{3}\right)\right) & =z^{2}+2 i x^{2} z+2 i y^{3} z \\
& + \begin{cases}\left(t_{1}+t_{2} y\right) x y^{4+q} & \text { if } p=2 q-1, \\
\left(t_{1}+t_{2} y\right) x^{2} y^{3+q} & \text { if } p=2 q .\end{cases}
\end{aligned}
$$

(ii) The Newton boundaries of the normal forms in table (9.1) except for $W_{1, p}^{\sharp}$ and of the normal form in 9.36 for $W_{1, p}^{\sharp}$ have each two 
compact $n$-dimensional faces $\sigma_{1}$ and $\sigma_{2}$. The following table lists the corresponding linear forms $l_{\sigma_{j}}$ and the value $s(f)$ from corollary 7.17 . A linear form is encoded by the values $\left(l_{\sigma_{j}}(x), l_{\sigma_{j}}(y), l_{\sigma_{j}}(z)\right)$.

$$
\begin{array}{llll}
W_{1, p}^{\sharp} & \sigma_{1}: \frac{1}{12}(3,2,6) & \sigma_{2}: \frac{1}{12+p}(3,2,6+p) & \frac{5}{12+p} \\
S_{1, p}^{\sharp} & \sigma_{1}: \frac{1}{10}(3,2,4) & \sigma_{2}: \frac{1}{10+p}(3,2,4+p) & \frac{5}{10+p} \\
U_{1, p} & \sigma_{1}: \frac{1}{9}(3,2,3) & \sigma_{2}: \frac{1}{9+p}(3+p, 2,3) & \frac{5}{9+p} \\
E_{3, p} & \sigma_{1}: \frac{1}{18}(6,2,9) & \sigma_{2}: \frac{1}{2(9+p)}(6+p, 2,9+p) & \frac{4}{9} \\
Z_{1, p} & \sigma_{1}: \frac{1}{14}(4,2,7) & \sigma_{2}: \frac{1}{2(7+p)}(4+p, 2,7+p) & \frac{3}{7} \\
Q_{2, p} & \sigma_{1}: \frac{1}{12}(4,2,5) & \sigma_{2}: \frac{1}{2(6+p)}(4+p, 2,5+p) & \frac{1}{2} \\
W_{1, p} & \sigma_{1}: \frac{1}{12}(3,2,6) & \sigma_{2}: \frac{1}{2(6+p)}(3+p, 2,6+p) & \frac{5}{12} \\
S_{1, p} & \sigma_{1}: \frac{1}{10}(3,2,4) & \sigma_{2}: \frac{1}{2(5+p)}(3+p, 2,4+p) & \frac{1}{2}
\end{array}
$$

Theorem 9.6. Consider the normal form in (9.36) for $W_{1, p}^{\sharp}$ and the normal forms in table (9.1) for the other seven series. Recall the notation $\omega_{0}:=d x d y d z$ from remark $7.14(v)$. Define

$$
\begin{aligned}
& b_{1}:=s\left(\omega_{0}, \alpha_{1}\right)(1,0) \in C^{\alpha_{1}}, \\
& b_{2}:=s\left(\omega_{0}, \beta_{1}\right)(1,0) \in C^{\beta_{1}}, \\
& b_{3}:=s\left(y \omega_{0}, \alpha_{2}\right)(1,0) \in C^{\alpha_{2}}, \\
& b_{4}:=s\left(y \omega_{0}, \beta_{2}\right)(1,0) \in C^{\beta_{2}} .
\end{aligned}
$$

If $m \mid p$, choose $b_{5} \in C^{\beta_{2}}$ with $\mathbb{C} \cdot b_{5}=\left\{v \in C^{\beta_{2}} \mid K_{f}^{(-2)}\left(b_{1}, v\right)=0\right\}$.

(a) All $b_{j} \neq 0$. And $K_{f}^{(-2)}\left(b_{1}+b_{2}, b_{3}+b_{4}\right)=0$. If $m \mid p$ then $C^{\beta_{2}}=$ $\mathbb{C} \cdot b_{4} \oplus \mathbb{C} \cdot b_{5}$.

(b) We write $t=\left(t_{1}, t_{2}\right)$. Recall the notation $\alpha\left(s[\omega]_{0}(t)\right)=$ $\min (\alpha \mid s(\omega, \alpha)(t) \neq 0)$ from remark 7.14 (iv).

$$
\begin{aligned}
\alpha\left(s\left[\omega_{0}\right]_{0}(t)\right)= & \alpha_{1}, \\
s\left(\omega_{0}, \alpha_{1}\right)(t)= & b_{1}, \\
s\left(\omega_{0}, \beta\right)(t)= & 0 \quad \text { for } \alpha_{1}<\beta<\beta_{1}, \\
s\left(\omega_{0}, \beta_{1}\right)(t)= & t_{1}^{1 / m_{2}} \cdot b_{2}, \\
s\left(\omega_{0}, \alpha_{2}\right)(t)= & \frac{t_{2}}{t_{1}} \cdot \frac{-1}{m_{2}} \cdot t_{1}^{-1 / m_{2}} \cdot b_{3}+s\left(\omega, \alpha_{2}\right)\left(t_{1}, 0\right), \\
s\left(\omega_{0}, \beta_{2}\right)(t) \quad & \begin{cases}=s\left(\omega_{0}, \beta_{2}\right)\left(t_{1}, 0\right) & \text { if } m \not p, \\
\in s\left(\omega_{0}, \beta_{2}\right)\left(t_{1}, 0\right)+\mathbb{C} \cdot b_{5} & \text { if } m \mid p,\end{cases}
\end{aligned}
$$


with

\begin{tabular}{l|l|l} 
& $s\left(\omega_{0}, \alpha_{2}\right)\left(t_{1}, 0\right)$ & $s\left(\omega_{0}, \beta_{2}\right)\left(t_{1}, 0\right)$ \\
\hline$\left(r_{I}=2 \& p \geq 2\right)$ or & & \\
$\left(r_{I}=1 \& p \geq 3\right)$ or $U_{1,2}$ & 0 & 0 \\
\hline$W_{1,1}^{\sharp}, S_{1,1}^{\sharp}, U_{1,1}$ & $c_{1} \cdot t_{1}^{2-1 / m_{2}} \cdot b_{3}$ & $c_{2} \cdot t_{1}^{2} \cdot b_{4}$ \\
\hline$W_{1,2}^{\sharp}, S_{1,2}^{\sharp}, E_{3,1}$ & & \\
$Z_{1,1}, Q_{2,1}, W_{1,1}, S_{1,1}$ & $c_{1} \cdot t_{1}^{1-1 / m_{2}} \cdot b_{3}$ & $c_{2} \cdot t_{1} \cdot b_{4}$
\end{tabular}

for some values $c_{1}, c_{2} \in \mathbb{C}$.

$$
\begin{aligned}
& \alpha\left(s\left[y \omega_{0}\right]_{0}(t)=\alpha_{2},\right. \\
& s\left(y \omega_{0}, \alpha_{2}\right)(t)=t_{1}^{-1 / m_{2}} \cdot b_{3} \text {, } \\
& s\left(y \omega_{0}, \beta_{2}\right)(t) \quad \begin{cases}=b_{4} & \text { if } m \not p \text { or } t_{2}=0, \\
\in b_{4}+\mathbb{C} \cdot b_{5} & \text { if } m \mid p,\end{cases} \\
& s\left(\sigma, \beta_{2}\right)(t) \begin{cases}=0 & \text { if } m \not p \\
\in \mathbb{C} \cdot b_{5} & \text { if } m \mid p\end{cases}
\end{aligned}
$$

for $\sigma \in H_{0}^{\prime \prime}\left(f_{t}\right)$ with $\alpha(\sigma)>\alpha_{2}$.

(c) In the five series with $r_{I}=2$ (see table (5.1) for $b \in \mathbb{Z}_{\geq 0}$

$$
\begin{aligned}
\alpha\left(s\left[y^{b+1} \omega_{0}\right]_{0}(t)\right) & =\alpha_{2}+\frac{2 b}{m_{2}}=\frac{2 b+1}{m_{2}}, \\
s\left(y^{b+1} \omega_{0}, \frac{2 b+1}{m_{2}}\right)(t) & =t_{1}^{-(2 b+1) / m_{2}} \cdot s\left(y^{b+1} \omega_{0}, \frac{2 b+1}{m_{2}}\right)(1,0) .
\end{aligned}
$$

Especially, if $p=m r$ then $\frac{2 r+1}{m_{2}}=\frac{1}{m}=\beta_{2}, b_{5}$ can be chosen as $b_{5}=$ $s\left(y^{r+1} \omega_{0}, \beta_{2}\right)(1,0)$, and

$$
s\left(y^{r+1} \omega_{0}, \beta_{2}\right)(t)=t_{1}^{-1 / m} \cdot b_{5} .
$$

(d) In the three subseries $W_{1,12 r}^{\sharp}, S_{1,10 r}^{\sharp}, U_{1,9 r}$ (i.e. the subseries with $r_{I}=1$ and $\left.m \mid p\right), b_{5}$ can be chosen such that $b_{5}$ and $\omega$ in the following table 9.54 satisfy

$$
\begin{aligned}
\alpha\left(s[\omega]_{0}(t)\right) & =\beta_{2}=\frac{1}{m}, \\
s\left(\omega, \beta_{2}+1\right)(t) & =t_{1}^{-1 / m} \cdot b_{5} .
\end{aligned}
$$




\begin{tabular}{r|l} 
& $\omega$ \\
\hline$W_{1,12+24 r}^{\sharp}, S_{1,10+20 r}^{\sharp}$ & $x y^{r} \omega_{0}$ \\
$U_{1,9+18 r}$ & $y^{r} z \omega_{0}$ \\
$W_{1,24 r}^{\sharp}, S_{1,20 r}^{\sharp}, U_{1,18 r}$ & $y^{r+1} \omega_{0}$
\end{tabular}

Proof: (a) Observe $\nu\left(\omega_{0}\right)-1=\alpha_{1}<s(f)$ and $\nu\left(y \omega_{0}\right)-1=\alpha_{2}<$ $s(f)$. This, theorem 7.16 and corollary 7.17 show (9.38), 9.45), $b_{1} \neq 0$ and $b_{3} \neq 0 . \quad b_{2} \neq 0$ will be shown below. (9.40) (which will also be shown below) and $K_{f}^{(-2)}\left(H_{0}^{\prime \prime}\left(f_{t}\right), H_{0}^{\prime \prime}\left(f_{t}\right)\right)=0$ give especially

$$
0=K_{f}^{(-2)}\left(s\left[\omega_{0}\right]_{0}(1,0), s\left[y \omega_{0}\right]_{0}(1,0)\right)=K_{f}^{(-2)}\left(b_{1}+b_{2}, b_{3}+b_{4}\right) .
$$

As $K_{f}^{(-2)}\left(b_{2}, b_{3}\right) \neq 0$, also $K_{f}^{(-2)}\left(b_{1}, b_{4}\right) \neq 0$ and $b_{4} \neq 0$ and in the case $m \mid p C^{\beta_{2}}=\mathbb{C} \cdot b_{4} \oplus \mathbb{C} \cdot b_{5}$.

(b)-(d) We restrict to the series $E_{3, p}$. The calculations for the series $Z_{1, p}, Q_{2, p}, W_{1, p}$ and $S_{1, p}$ are very similar. The calculations for the series $W_{1, p}^{\sharp}, S_{1, p}^{\sharp}$ and $U_{1, p}$ are similar, but require more case discussions.

The two compact faces $\sigma_{1}$ and $\sigma_{2}$ (remark 9.5) of the Newton boundary give rise to the following two relations

$$
\begin{aligned}
\frac{1}{3} x f_{x}+\frac{1}{9} y f_{y}+\frac{1}{2} z f_{z}-\frac{p}{9} t_{1} y^{9+p}-\frac{p+1}{9} t_{2} y^{10+p} & =f \\
\frac{6+p}{2(9+p)} x f_{x}+\frac{2}{2(9+p)} y f_{y}+\frac{1}{2} z f_{z} & \\
-\frac{p}{2(9+p)} x^{3}-\frac{1}{9+p} t_{2} y^{10+p} & =f .
\end{aligned}
$$

These relations and (7.24) give the following two values for $\partial_{\tau} \tau s\left[x^{a} y^{b} \omega_{0}\right]_{0}(t)$

$$
\begin{aligned}
& \partial_{\tau} \tau s\left[x^{a} y^{b} \omega_{0}\right]_{0}(t) \\
= & l_{\sigma_{1}}(a+1, b+1,1) \cdot s\left[x^{a} y^{b} \omega_{0}\right]_{0}(t) \\
& -\frac{p}{9} t_{1} \partial_{\tau} s\left[x^{a} y^{b+9+p} \omega_{0}\right]_{0}(t)-\frac{p+1}{9} t_{2} \partial_{\tau} s\left[x^{a} y^{b+10+p} \omega_{0}\right]_{0}(t) \\
= & l_{\sigma_{2}}(a+1, b+1,1) \cdot s\left[x^{a} y^{b} \omega_{0}\right]_{0}(t) \\
& -\frac{p}{2(9+p)} \partial_{\tau} s\left[x^{a+3} y^{b} \omega_{0}\right]_{0}(t)-\frac{1}{9+p} t_{2} \partial_{\tau} s\left[x^{a} y^{b+10+p} \omega_{0}\right]_{0}(t) .
\end{aligned}
$$


This gives for any $\beta$ with $\operatorname{dim} C^{\beta} \neq 0$

$$
\begin{aligned}
& \left(\beta+1-l_{\sigma_{1}}(a+1, b+1,1)\right) s\left(x^{a} y^{b} \omega_{0}, \beta\right)(t) \\
= & -\frac{p}{9} t_{1} \partial_{\tau} s\left(x^{a} y^{b+9+p} \omega_{0}, \beta+1\right)(t) \\
& -\frac{p+1}{9} t_{2} \partial_{\tau} s\left(x^{a} y^{b+10+p} \omega_{0}, \beta+1\right)(t), \\
& \left(\beta+1-l_{\sigma_{2}}(a+1, b+1,1)\right) s\left(x^{a} y^{b} \omega_{0}, \beta\right)(t) \\
= & -\frac{p}{2(9+p)} \partial_{\tau} s\left(x^{a+3} y^{b} \omega_{0}, \beta+1\right)(t \\
& -\frac{1}{9+p} t_{2} \partial_{\tau} s\left(x^{a} y^{b+10+p} \omega_{0}, \beta+1\right)(t) .
\end{aligned}
$$

Furthermore, 7.39 gives

$$
\begin{aligned}
\partial_{t_{1}} s\left[x^{a} y^{b} \omega_{0}\right]_{0}(t) & =\left(-\partial_{\tau}\right) s\left[x^{a} y^{b+9+p} \omega_{0}\right]_{0}(t) \\
\partial_{t_{2}} s\left[x^{a} y^{b} \omega_{0}\right]_{0}(t) & =\left(-\partial_{\tau}\right) s\left[x^{a} y^{b+10+p} \omega_{0}\right]_{0}(t) \\
& =\partial_{t_{1}} s\left[x^{a} y^{b+1} \omega_{0}\right]_{0}(t) .
\end{aligned}
$$

(9.59)-9.62) give

$$
\begin{array}{r}
\left(\frac{p}{9} t_{1} \partial_{t_{1}}+\frac{p+1}{9} t_{2} \partial_{t_{2}}-(\beta+1)+l_{\sigma_{1}}(a+1, b+1,1)\right) \\
s\left(x^{a} y^{b} \omega_{0}, \beta\right)(t)=0 \\
\left(\frac{1}{9+p} t_{2} \partial_{t_{2}}-(\beta+1)+l_{\sigma_{2}}(a+1, b+1,1)\right) s\left(x^{a} y^{b} \omega_{0}, \beta\right)(t) \\
=\frac{p}{2(9+p)} \partial_{\tau} s\left(x^{a+3} y^{b} \omega_{0}, \beta+1\right)(t) .
\end{array}
$$

(9.63) gives for $t_{2}=0$

$$
s\left(x^{a} y^{b} \omega_{0}, \beta\right)\left(t_{1}, 0\right)=t_{1}^{\frac{9}{p}\left(\beta+1-l_{\sigma_{1}}(a+1, b+1,1)\right)} \cdot s\left(x^{a} y^{b} \omega_{0}, \beta\right)(1,0)
$$

The following eight equations are special cases of (9.65).

$$
\begin{aligned}
s\left(\omega_{0}, \alpha_{1}\right)\left(t_{1}, 0\right) & =b_{1}, \\
s\left(\omega_{0}, \beta_{1}\right)\left(t_{1}, 0\right) & =t_{1}^{1 / m_{2}} \cdot b_{2}, \\
s\left(\omega_{0}, \alpha_{2}\right)\left(t_{1}, 0\right) & =t_{1}^{-1 / m_{2}+1 / p} \cdot s\left(\omega_{0}, \alpha_{2}\right)(1,0), \\
s\left(\omega_{0}, \beta_{2}\right)\left(t_{1}, 0\right) & =t_{1}^{1 / p} \cdot s\left(\omega_{0}, \beta_{2}\right)(1,0),
\end{aligned}
$$


TORELLI RESULTS FOR MARKED BIMODAL SINGULARITIES

$$
\begin{aligned}
s\left(y^{b+1} \omega_{0}, \frac{2 b+1}{m_{2}}\right)\left(t_{1}, 0\right) & =t_{1}^{-(2 b+1) / m_{2}} \cdot s\left(y^{b+1} \omega_{0}, \frac{2 b+1}{m_{2}}\right)(1,0), \\
s\left(y \omega_{0}, \alpha_{2}\right)\left(t_{1}, 0\right) & =t_{1}^{-\alpha_{2}} \cdot b_{3}=t_{1}^{-1 / m_{2}} \cdot b_{3}, \\
s\left(y \omega_{0}, \beta_{2}\right)\left(t_{1}, 0\right) & =b_{4}, \\
s\left(y^{r+1} \omega_{0}, \beta_{2}\right)\left(t_{1}, 0\right) & =t_{1}^{-1 / m_{2}} \cdot s\left(y^{r+1} \omega_{0}, \beta_{2}\right)(1,0) \quad \text { if } p=18 r .(9.73)
\end{aligned}
$$

Claim: Fix some $b \in \mathbb{Z}_{\geq 0}$.

(i) $\nu\left(y^{b+1} \omega_{0}\right)=\alpha_{2}+\frac{b}{9+p}=\frac{2 b+1}{m_{2}}$.

(ii) Any $(n+1)$-form $d f \wedge d \eta$ which contains $y^{b+1} \omega_{0}$ as a summand, contains a summand $g \cdot \omega_{0}$ with $g$ a monomial (times a nonzero scalar) with $\nu\left(g \cdot \omega_{0}\right) \leq \nu\left(y^{b+1} \omega_{0}\right)$.

(iii) $\bar{\nu}\left(y^{b+1} \omega_{0}\right)=\frac{2 b+1}{m_{2}}$.

Proof of the claim: (i) Trivial. (iii) follows from (i) and (ii).

(iii) The only monomial differential $(n-1)$-forms $\eta$ such that $d f \wedge d \eta$ contains $f_{y} \cdot y^{c} \cdot \omega_{0}$ are $\eta_{1}=-x y^{c} d z$ and $\eta_{2}=y^{c} z d x$, and

$$
\begin{aligned}
& d f \wedge d \eta_{1}=f_{y} \cdot y^{c} \cdot \omega_{0}-f_{x} \cdot c \cdot x y^{c-1} \cdot \omega_{0}, \\
& d f \wedge d \eta_{2}=f_{y} \cdot y^{c} \cdot \omega_{0}-f_{z} \cdot c \cdot y^{c-1} z \cdot \omega_{0} .
\end{aligned}
$$

These $(n+1)$-forms contain $(3-2 c) x^{2} y^{c+2} \omega_{0}$ respectively $3 x^{2} y^{c+2} \omega_{0}$, and

$$
\nu\left(x^{2} y^{c+2} \omega_{0}\right) \leq \nu\left(y^{c+8+p} \omega_{0}\right)
$$

The claim and theorem 7.16 imply

$$
\begin{aligned}
\alpha\left(s\left[y^{b+1} \omega_{0}\right]_{0}(t)\right) & =\frac{2 b+1}{m_{2}}, \\
s\left(y^{b+1} \omega_{0}, \frac{2 b+1}{m_{2}}\right)(t) & \neq 0 .
\end{aligned}
$$

Especially, $b_{3} \neq 0$, and if $p=18 r$ also $s\left(y^{r+1} \omega_{0}, \beta_{2}\right)(t) \neq 0$. In this case $p=18 r$, the vanishing

$$
K_{f}^{(-2)}\left(s\left[\omega_{0}\right]_{0}(1,0), s\left[y^{r+1} \omega_{0}\right]_{0}(1,0)\right)=0
$$

gives $K_{f}^{(-2)}\left(b_{1}, s\left(y^{r+1} \omega_{0}, \beta_{2}\right)(1,0)\right)=0$. Therefore in this case we can choose $b_{5}=s\left(y^{r+1} \omega_{0}, \beta_{2}\right)(1,0)$.

The elementary sections $s\left(y^{b+1} \omega_{0}, \frac{2 b+1}{m_{2}}\right)(t)$ are independent of $t_{2}$ because 9.62 gives

$$
\partial_{t_{2}} s\left(y^{b+1} \omega_{0}, \frac{2 b+1}{m_{2}}\right)(t)=\partial_{t_{1}} s\left(y^{b+2} \omega_{0}, \frac{2 b+1}{m_{2}}\right)(t)=0 .
$$


Now part (c), i.e. $9.49-(9.51)$, and $(9.46)$ are proved.

(9.62) gives also

$$
\begin{aligned}
\partial_{t_{2}} s\left[\omega_{0}\right]_{0}(t) & =\partial_{t_{1}} s\left[y \omega_{0}\right]_{0}(t), \\
\text { so } s\left(\omega_{0}, \beta\right)(t) & =s\left(\omega_{0}, \beta\right)\left(t_{1}, 0\right) \quad \text { for } \alpha_{1} \leq \beta<\alpha_{2} .
\end{aligned}
$$

With 9.66 and 9.59 and 9.75 we obtain

$$
s\left(\omega_{0}, \beta\right)\left(t_{1}, 0\right)= \begin{cases}b_{1} & \text { if } \beta=\alpha_{1}, \\ \frac{-p}{9\left(\beta-\alpha_{1}\right)} t_{1} \partial_{\tau} s\left(y^{9+p} \omega_{0}, \beta+1\right)\left(t_{1}, 0\right)=0 & \text { if } \alpha_{1}<\beta<\beta_{1}, \\ \frac{-p}{9\left(\beta_{1}-\alpha_{1}\right)} t_{1} \partial_{\tau} s\left(y^{9+p} \omega_{0}, \beta_{1}+1\right)\left(t_{1}, 0\right) \neq 0 & \text { if } \beta=\beta_{1} .\end{cases}
$$

This gives $b_{2} \neq 0$ and (together with (9.66) and (9.67) $(9.39)-(9.41)$.

The argument in the proof of part (a) with $K_{f}^{(-2)}\left(H_{0}^{\prime \prime}\left(f_{t}\right), H_{0}^{\prime \prime}\left(f_{t}\right)\right)=0$ gives $b_{4} \neq 0$ and $(9.47)$ and (9.48).

It rests to show (9.42)-(9.44). From (9.76), (9.46) and (9.47) we obtain

$$
\begin{aligned}
& \partial_{t_{2}} s\left(\omega_{0}, \alpha_{2}\right)(t)=\partial_{t_{1}} s\left(y \omega_{0}, \alpha_{2}\right)(t)=\partial_{t_{1}}\left(t_{1}^{-1 / m_{2}} \cdot b_{3}\right), \\
& \partial_{t_{2}} s\left(\omega_{0}, \beta_{2}\right)(t)=\partial_{t_{1}} s\left(y \omega_{0}, \beta_{2}\right)(t) \begin{cases}=0 & \text { if } m \not p, \\
\in \mathbb{C} \cdot b_{5} & \text { if } m \mid p,\end{cases}
\end{aligned}
$$

which gives $(9.42)$ and $(9.43)$.

For (9.44) observe the following. The sections

$$
\begin{aligned}
& s\left(y \omega_{0}, \alpha_{2}\right)\left(t_{1}, 0\right)=t_{1}^{-1 / m_{2}} \cdot b_{3}, \\
& s\left(y \omega_{0}, \beta_{2}\right)\left(t_{1}, 0\right)=b_{4},
\end{aligned}
$$

and in the case $m \mid p \quad s\left(y^{r+1} \omega_{0}, \beta_{2}\right)\left(t_{1}, 0\right)=t_{1}^{-1 / m} \cdot b_{5}$

are univalued nowhere vanishing sections in the bundles $\bigcup_{t_{1} \in T} C^{\alpha_{2}}\left(t_{1}, 0\right)$ and $\bigcup_{t_{1} \in T} C^{\beta_{2}}\left(t_{1}, 0\right)$, and they generate these bundles. Also $s\left(\omega_{0}, \alpha_{2}\right)\left(t_{1}, 0\right)$ and $s\left(\omega_{0}, \beta_{2}\right)\left(t_{1}, 0\right)$ are univalued sections in these bundles. (9.68) and (9.69) show for $p \geq 2$ that they are everywhere vanishing. For $p=1$ they give the statement for $E_{3,1}$ in the last line of table (9.44). This finishes the proof of the parts (b) and (c) for the series $E_{3, p}$.

Proof of $M_{T}^{m_{2}}=\mathrm{id}$ :

By theorem 9.6, the following sections in the bundles $\bigcup_{t_{1} \in T} C^{\beta}\left(t_{1}, 0\right)$ for $\beta$ as in table (9.77) are univalued nowhere vanishing sections and 
generate these bundles (in the case $\beta=\alpha_{1}$ only if $m \not p$ ).

\begin{tabular}{l|l|l|l|l|l} 
section & $b_{1}$ & $t_{1}^{1 / m_{2}} \cdot b_{2}$ & $t_{1}^{-1 / m_{2}} \cdot b_{3}$ & $b_{4}$ & $t_{1}^{-1 / m} \cdot b_{5}$ if $m \mid p$ \\
\hline$\beta$ & $\alpha_{1}$ & $\beta_{1}$ & $\alpha_{2}$ & $\beta_{2}$ & $\beta_{2}$ \\
\hline eigenvalue of & 1 & $e^{-2 \pi i / m_{2}}$ & $e^{2 \pi i / m_{2}}$ & 1 & $e^{2 \pi i / m}$ \\
$M_{T}$ on $\mathbb{C} \cdot b_{j}$ & & & &
\end{tabular}

Therefore $b_{1}$ and $b_{4}$ are univalued, and $b_{2}$ and $b_{3}$ (and $b_{5}$ if $m \mid p$ ) are multivalued flat sections with eigenvalues of $M_{T}$ as in the table. Thus $M_{T}^{m_{2}}$ is on $C^{\alpha_{1}}, C^{\beta_{1}}, C^{\alpha_{2}}, C^{\beta_{2}}, M l_{\zeta}$ and $M l_{e^{2 \pi i / m_{2}}}$ the identity. We will show that it is the identity on all of $M l$.

Consider firstly the case $m \not p$. Then by (9.24) $M_{T}^{m_{2}}$ is in

$\{\mathrm{id}\} \quad$ in the cases $W_{1,2 q-1}^{\sharp}, S_{1,2 q-1}^{\sharp}, U_{1,2 q}, E_{3, p}, Z_{1, p}$,

$\left\{\mathrm{id},\left.\mathrm{id}\right|_{B_{1}} \times\left.\left(-M_{h}^{m_{2} / 2}\right)\right|_{B_{2}}\right\} \quad$ in the cases $W_{1,2 q}^{\sharp}, S_{1,2 q}^{\sharp}, U_{1,2 q-1}$,

$\left\{\mathrm{id},\left.\left(-M_{h}^{m / 2}\right)\right|_{B_{1}} \times\left.\mathrm{id}\right|_{B_{2}}\right\} \quad$ in the cases $Q_{2, p}, W_{1, p}, S_{1, p}$.

On the other hand, in the cases in the second and third line of (9.78), $m_{2}=m+r_{I} p$ is even, and $M_{T}$ itself is in $G_{\mathbb{Z}}$ which is given by (5.6) in theorem 5.1. Thus $M_{T}^{m_{2}}=\mathrm{id}$ also in the second and third line of (9.78).

Consider secondly the case $m \mid p$, so $p=m r$. By (5.9) in theorem 5.1, $M_{T}^{m_{2}}=\varepsilon \cdot M_{h}^{k}$ for some $\varepsilon \in\{ \pm 1\}$ and some $k \in \mathbb{Z}$. Then $\varepsilon \cdot \zeta^{k}=1$ and $\varepsilon \cdot e^{2 \pi i k / m_{2}}=1$. If $\varepsilon=1$, then the two conditions boil down to $m \mid k$ and $m_{2} \mid k$, so to $m_{2} \mid k$. Then $M_{T}^{m_{2}}=\mathrm{id}$. If $\varepsilon=-1$, we will come below to a contradiction. Then the two conditions require $m$ even and $m_{2}$ even.

For each eigenvalue $\lambda$ of $M_{h}$ on $M l$ with $\operatorname{dim} M l_{\lambda}=1$, an eigenvector in $M l_{\lambda, \mathbb{Z}[\lambda]}$ exists. Then $M_{T}$ has an eigenvalue in $\operatorname{Eiw}(\lambda)$ on this eigenvector, and $M_{T}^{m_{2}}$ has the eigenvalue 1 on this eigenvector. Here $m_{2}$ even is used. Therefore $M_{T}^{m_{2}}=\mathrm{id}$ on $M l_{\lambda}$ for each

$$
\lambda \in\left\{\zeta, e^{2 \pi i / m_{2}}\right\} \cup\left\{\widetilde{\lambda} \mid \operatorname{dim} M l_{\tilde{\lambda}}=1\right\} .
$$

Comparison with table (5.1) shows that no $k \in \mathbb{Z}$ with $-\lambda^{k}=1$ for all these $\lambda$ exists. This gives a contradiction. The case $\varepsilon=-1$ is impossible. $M_{T}^{m_{2}}=\mathrm{id}$ is proved in all cases.

Proof that $M_{T}$ has the eigenvalues 1 and $\bar{\zeta}$ on $M l_{\zeta}$ and on $C^{\alpha_{1}}$ :

By table $(9.77), M_{T}$ has on $C^{\beta_{2}}$ and on $H_{e^{-2 \pi i \beta_{2}}}^{\infty}=H_{\bar{\zeta}}^{\infty}$ the eigenvalues 1 and $\zeta$. As $M l_{\zeta}$ is dual to $H_{\bar{\zeta}}^{\infty}$ and $H_{\zeta}^{\infty}$ is complex conjugate to $H_{\bar{\zeta}}^{\infty}, M_{t}$ has on $M l_{\zeta}, H_{\zeta}^{\infty}=H_{e^{-2 \pi i \alpha_{1}}}^{\infty}$ and $C^{\alpha_{1}}$ the eigenvalues 1 and $\bar{\zeta}$.

Proof of theorem 9.2 (a)+(b)+(d): 
(a) This follows immediately from (9.7), (9.9) and lemma (9.4) (c).

(b) All of this follows by carefully putting together the results in theorem 9.6. Here $v_{1}^{0}=b_{1}, v_{2}^{0}=b_{2}, v_{4}^{0} \in \mathbb{C}^{*} \cdot b_{4}$ suitable, and the section in the brackets on the right hand side of $(9.10)$ is

$$
\begin{array}{r}
s\left[\omega_{0}\right]_{0}(t)+\left(\frac{1}{m} \frac{t_{2}}{t_{1}}+\left\{\begin{array}{c}
0 \\
-c_{1} \cdot t_{1}^{2} \\
-c_{1} \cdot t_{1}
\end{array}\right\}\right) \cdot s\left[y \omega_{0}\right]_{0}(t) \\
\bmod \bigoplus_{\alpha_{2}<\beta<\beta_{2}} C^{\beta} \oplus \mathbb{C} \cdot b_{5} \oplus V^{>\beta_{2}} .
\end{array}
$$

The three cases in $\{\ldots\}$ correspond to the three lines in 9.44 . The linear combination is chosen such that it has no part in $C^{\alpha_{2}}$. This section and the fact $K_{f}^{(-2)}\left(H_{0}^{\prime \prime}\left(f_{t}\right), H_{0}^{\prime \prime}\left(f_{t}\right)\right)=0$ determine $H_{0}^{\prime \prime}\left(f_{t}\right)$. By table (9.77), $M_{T}$ has on $v_{1}^{0}=b_{4}$ the eigenvalue 1 .

(c) Consider the coordinate change

$$
\varphi:\left(\mathbb{C}^{3}, 0\right) \rightarrow\left(\mathbb{C}^{3}, 0\right), \quad(x, y, z) \mapsto(x, y,-z)
$$

We treat the cases $U_{1,9+18 r}$ and $U_{1,18 r}$ separately.

The case $U_{1,9+18 r}$ : Then $\varphi \in G_{\mathcal{R}}^{\text {smar,gen }} \subset G^{\text {smar }}$, and

$$
\varphi^{*}\left(\omega_{0}\right)=-\omega_{0}, \quad \varphi^{*}\left(y^{r} z \omega_{0}\right)=y^{r} z \omega_{0} .
$$

Now compare (9.39) and (9.54) $\varphi \varphi$ induces an automorphism $(\varphi)_{c o h}$ on $C^{\alpha_{1}}$ and $C^{\beta_{2}}$ with

$$
(\varphi)_{c o h}\left(b_{1}\right)=-b_{1}, \quad(\varphi)_{c o h}\left(b_{4}\right)=-b_{4}, \quad(\varphi)_{c o h}\left(b_{5}\right)=b_{5}
$$

One can choose $g_{3}=-M_{T} \circ(\varphi)_{h o m} \in G^{\text {mar }}$.

The case $U_{1,18 r}$ : Because of (9.54) and (9.77), instead of (9.81) the identities

$$
\varphi^{*}\left(\omega_{0}\right)=-\omega_{0}, \quad \varphi^{*}\left(y^{r+1} \omega_{0}\right)=-y^{r+1} \omega_{0}
$$

are relevant. Now $(\varphi)_{\text {coh }}$ is because of $(9.15)$ an isomorphism

$$
H_{0}^{\prime \prime}\left(f_{\left(t_{1}, 0\right)}\right) \rightarrow H_{0}^{\prime \prime}\left(f_{\left(-t_{1}, 0\right)}\right), \quad C^{\beta_{2}}\left(t_{1}, 0\right) \rightarrow C^{\beta_{2}}\left(-t_{1}, 0\right) .
$$

The composition

(- id) $\circ$ (math. pos. flat shift from $C^{\beta_{2}}\left(-t_{1}, 0\right)$ to $\left.C^{\beta_{2}}\left(t_{1}, 0\right)\right) \circ(\varphi)_{c o h}$ 
acts on $C^{\beta_{2}}\left(t_{1}, 0\right)$ and has because of $(9.76)$ the eigenvectors $b_{4}$ and $b_{5}$ with the eigenvalues 1 and $e^{\pi i / 9}$ :

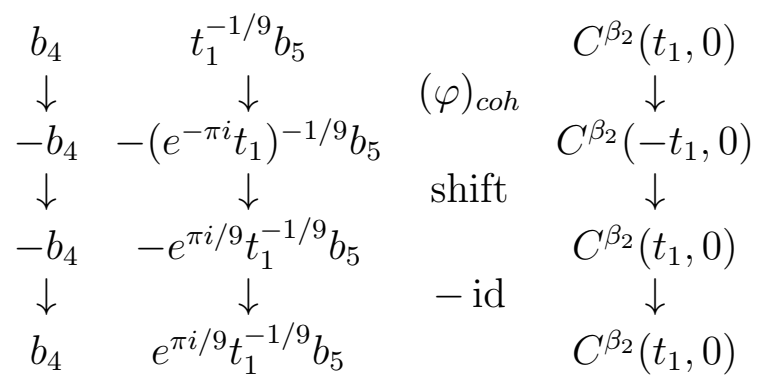

The corresponding composition

(-id) $\circ$ (math. pos. flat shift from $M l\left(f_{\left(-t_{1}, 0\right)}\right)$ to $\left.M l\left(f_{\left(t_{1}, 0\right)}\right)\right) \circ(\varphi)_{h o m}$

is in $G^{m a r}$ and can be chosen as $g_{3}$.

\section{Period maps And Torelli Results For THE QUADRANGle SINGULARITIES}

In this section we will prove for the quadrangle singularities the strong global Torelli conjecture 8.11 (a), the conjectures 8.6 (b) - id $\notin G^{\text {smar }}$ and (a) $G_{\mathbb{Z}}=G^{\text {mar }}$. The Torelli conjecture for the unmarked singularities had been proved in He93 (and the proof had been sketched in [He95]). The main new ingredient for the Torelli result for marked singularities is a much stronger control of the group $G_{\mathbb{Z}}$, in theorem 6.1. But we will also recall the old ingredients from [He93], the space $D_{B L}$ and a period map for which we need calculations of the Gauss-Manin connection.

The six bimodal families of quadrangle singularities have as surface singularities the normal forms $f_{\left(t_{1}, t_{2}\right)}$ in table (10.1). These are not the normal forms in [AGV85, 15.1]. We will justify the normal forms and explain their properties after theorem 10.1 . The parameters $\left(t_{1}, t_{2}\right)$ are in $T^{(5)}:=(\mathbb{C}-\{0,1\}) \times \mathbb{C}$. Table $(10.1)$ lists additionally weights $\left(w_{x}, w_{y}, w_{z}\right)$ such that $f_{\left(t_{1}, 0\right)}$ is quasihomogeneous of weighted degree 1 and two numbers $m_{0}$ and $m_{\infty}$ We set $m_{1}:=m_{0}$. Observe $w_{y}=\frac{2}{m}<$ $w_{x} \leq w_{z}$. 


\begin{tabular}{lllll} 
& & $\left(w_{x}, w_{y}, w_{z}\right)$ & $m_{0}$ & $m_{\infty}$ \\
\hline$W_{1,0}$ & $x^{4}+\left(4 t_{1}-2\right) x^{2} y^{3}+y^{6}+t_{2} x^{2} y^{4}+z^{2}$ & $\left(\frac{1}{4}, \frac{1}{6}, \frac{1}{2}\right)$ & 12 & 6 \\
$S_{1,0}$ & $x^{2} z+y^{3} z+y z^{2}+t_{1} x^{2} y^{2}+t_{2} x^{2} y^{3}$ & $\left(\frac{3}{10}, \frac{2}{10}, \frac{4}{10}\right)$ & 10 & 5 \\
$U_{1,0}$ & $x z(x-z)+y^{3}\left(x-t_{1} z\right)+t_{2} y^{4} z$ & $\left(\frac{1}{3}, \frac{2}{9}, \frac{1}{3}\right)$ & 9 & 9 \\
$E_{3,0}$ & $x\left(x-y^{3}\right)\left(x-t_{1} y^{3}\right)+t_{2} x^{2} y^{4}+z^{2}$ & $\left(\frac{1}{3}, \frac{1}{9}, \frac{1}{2}\right)$ & 9 & 9 \\
$Z_{1,0}$ & $x y\left(x-y^{2}\right)\left(x-t_{1} y^{2}\right)+t_{2} x^{2} y^{4}+z^{2}$ & $\left(\frac{2}{7}, \frac{1}{7}, \frac{1}{2}\right)$ & 7 & 7 \\
$Q_{2,0}$ & $x\left(x-y^{2}\right)\left(x-t_{1} y^{2}\right)+y z^{2}+t_{2} x z^{2}$ & $\left(\frac{1}{3}, \frac{1}{6}, \frac{5}{12}\right)$ & 6 & 6
\end{tabular}

Recall that table (6.1) lists for these singularities the Milnor number $\mu$, the characteristic polynomials $b_{j}, j \geq 1$, of the monodromy on the Orlik blocks $B_{j}$ in theorem 5.1 , the order $m$ of the monodromy and the index $r_{I}$.

For each 2-parameter family in table 10.1), we choose $f_{0}:=f_{(i, 0)}$ as reference singularity. And as in section $9, M_{\mu}^{\text {mar }},\left(M_{\mu}^{\text {mar }}\right)^{0}, G_{\mathbb{Z}}, G^{\text {mar }}$, $M l, H^{\infty}$ and $C^{\alpha}$ mean the objects for $f_{0}$. As always, $\zeta:=e^{2 \pi i / m}$.

We will construct branched coverings $c^{(2)}$ and $c^{(6)}$ and unbranched coverings $c^{(1)}$ and $c^{(5)}$ as follows.

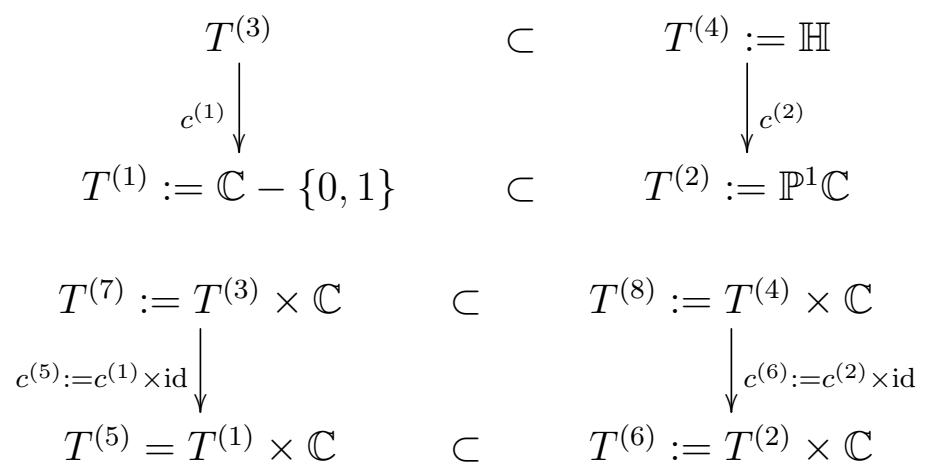

Let $\Gamma \subset P G L(2, \mathbb{R})$ be a triangle group of type $\left(\frac{1}{m_{0}}, \frac{1}{m_{1}}, \frac{1}{m_{\infty}}\right)$. The quotient $\mathbb{H} / \Gamma$ is an orbifold with three orbifold points of orders $m_{0}, m_{1}$ and $m_{\infty}$. They are the images of the elliptic fixed points of $\Gamma$ on $T^{(4)}=\mathbb{H}$ of orders $m_{0}, m_{1}$ and $m_{\infty}$. As a manifold $\mathbb{H} / \Gamma \cong \mathbb{P}^{1} \mathbb{C}$. Choose coordinates on $\mathbb{H} / \Gamma$ such that 0 and 1 are orbifold points of order $m_{0}=m_{1}$ and $\infty$ is an orbifold point of order $m_{\infty}$. Denote by

$$
c^{(2)}: T^{(4)}=\mathbb{H} \rightarrow T^{(2)}=\mathbb{P}^{1} \mathbb{C}
$$

the quotient map. It is a branched covering. Denote

$$
\begin{aligned}
T^{(3)} & :=T^{(4)}-\left(c^{(2)}\right)^{-1}(\{0,1, \infty\}), \\
\text { and } \quad c^{(1)} & :=\left.c^{(2)}\right|_{T^{(4)}}: T^{(3)} \rightarrow T^{(1)} .
\end{aligned}
$$

It is a covering. 
Theorem 10.1. Consider a bimodal family of quadrangle surface singularities in table (10.1).

(a) There are canonical isomorphisms

$$
T^{(7)} \rightarrow\left(M_{\mu}^{\text {smar }}\right)^{0} \rightarrow\left(M_{\mu}^{\text {mar }}\right)^{0} .
$$

(b) - id $\notin G^{\text {smar }}$, where $G^{\text {smar }}$ is the group for the singularities of multiplicity $\geq 3$, namely the curve singularities $W_{1,0}, E_{3,0}, Z_{1,0}$ and the surface singularities $S_{1,0}, U_{1,0}, Q_{2,0}$. So, conjecture 8.6 (b) is true.

(c) $G_{\mathbb{Z}}=G^{\text {mar }}$. So, $M_{\mu}^{\text {mar }}=\left(M_{\mu}^{\text {mar }}\right)^{0}$, and conjecture 8.6 (a) is true.

(d) The period map BL: $M_{\mu}^{\text {mar }} \rightarrow D_{B L}$ is an embedding. So, the strong global Torelli conjecture 8.11 (a) is true.

The Torelli result for unmarked singularities (the period map $M_{\mu}^{\text {mar }} / G_{\mathbb{Z}} \rightarrow D_{B L} / G_{\mathbb{Z}}$ is an embedding) was proved already in [He93], and also that there is a well defined period map $T^{(7)} \rightarrow D_{B L}$ and that it is an embedding. But we prefer to give an independent account and recover these results. The hardest part is in any case new. It is the precise control of $G_{\mathbb{Z}}$ in theorem 6.1.

First we discuss the normal forms in table (10.1) and the right equivalence classes in them.

Each bimodal family of quadrangle surface singularities contains a 1-parameter subfamily of quasihomogeneous singularities. The exceptional set of the minimal good resolution of such a singularity consists of 5 smooth rational curves. One, the central curve, intersects each of the other 4, the branches, in one point. The right equivalence class of one quasihomogeneous surface singularity is determined by the central curve with the 4 intersection points and the self intersection numbers of the 4 branches. Table (10.6) lists these self intersection numbers.

$$
\begin{array}{llllll}
W_{1,0} & S_{1,0} & U_{1,0} & E_{3,0} & Z_{1,0} & Q_{2,0} \\
(2,2,3,3) & (2,2,3,4) & (2,3,3,3) & (2,2,2,3) & (2,2,2,4) & (2,2,2,5)
\end{array}
$$

In table (10.1), the singularities with $t_{2}=0$ are quasihomogeneous. Their normal forms are not taken from [AGV85, 15.1], but from [Bi92, Anhang A2, p. 191]. They are chosen such that the cross ratio of the 4 intersection points on the central curve has $j$-invariant $j=\frac{4}{27} \frac{\left(t_{1}^{2}-t_{1}+1\right)^{3}}{t_{1}^{2}\left(1-t_{1}\right)^{2}}$. This fact implies that the families in table (10.1) contain representatives of all right equivalence classes in one $\mu$-homotopy class.

From the weights (or the spectral numbers, see below theorem 10.6 one deduces easily that any monomial basis of the Jacobi algebra of one 
quasihomogeneous surface singularity $f_{t_{1}, 0}$ contains precisely one monomial $p_{>1}$ of weighted degree $>1$ and that $\operatorname{deg}_{w} p_{>1}=1+\frac{2}{m}=1+w_{y}$. AGV85, 12.6 Theorem] says here that any semiquasihomogeneous singularity with quasihomogeneous part $f_{t_{1}, 0}$ is right equivalent to $f_{t_{1}, 0}+t_{2} \cdot p_{>1}$ for some $t_{2} \in \mathbb{C}$. In table (10.1) we have chosen the monomial $p_{>1}$ such that it is part of a monomial basis of the Jacobi algebra of $f_{t_{1}, 0}$ for any $t_{1} \in T^{(1)}$.

Remarks 10.2. It is nontrivial (and slightly surprising) that such a monomial $p_{>1}$ exists simultaneously for all $t_{1} \in T^{(1)}$. In [He93] He95] the second author had overlooked this problem and had chosen in the four cases $S_{1,0}, E_{3,0}, Z_{1,0}, Q_{2,0}$ a monomial which does not work for special parameters $t_{1} \in T^{(1)}$. The following table 10.8 lists for all 6 families all monomials $\widetilde{p}$ of weighted degree $1+\frac{2}{m}$ and for each of them the function $q\left(t_{1}\right)$ with

$$
\widetilde{p} \equiv q\left(t_{1}\right) \cdot p_{>1} \quad \bmod \left(\text { Jacobi ideal of } f_{t_{1}, 0}\right) \text {, }
$$

where $p_{>1}=\frac{\partial f_{\left(t_{1}, t_{2}\right)}}{\partial t_{2}}$ is the monomial chosen in table 10.1 .

$$
\begin{array}{lllll} 
& p_{>1} & \widetilde{p}: q\left(t_{1}\right) & \widetilde{p}: q\left(t_{1}\right) & \widetilde{p}: q\left(t_{1}\right) \\
W_{1,0} & x^{2} y^{4} & x^{4} y: 1-2 t_{1} & y^{7}: 1-2 t_{1} & x^{2} y z: 0 \\
& & y^{4} z: 0 & y z^{2}: 0 & \\
S_{1,0} & x^{2} y^{3} & x^{2} y z:-t_{1} & y^{4} z:-t_{1} & y^{2} z^{2}: t_{1} \\
& & y^{6}: 2 t_{1}-1 & x^{4}: 2 t_{1}-1 & z^{3}: t_{1}\left(2 t_{1}-3\right) \\
U_{1,0} & y^{4} z & x^{2} y z:-t_{1} & x y z^{2}:-t_{1} & x y^{4}: t_{1} \\
& & x^{3} y: t_{1}\left(t_{1}-2\right) & y z^{3}: 1-2 t_{1} & \\
E_{3,0} & x^{2} y^{4} & x^{3} y: \frac{t_{1}+1}{2} & x y^{7}: \frac{t_{1}+1}{2 t_{1}} & y^{10}: \frac{t_{1}^{2}-t_{1}+1}{t_{1}^{2}} \\
& & y z^{2}: 0 & & \\
Z_{1,0} & x^{2} y^{4} & x^{3} y^{2}: \frac{t_{1}+1}{2} & x y^{6}: \frac{t_{1}+1}{2 t_{1}} & y^{8}: \frac{t_{1}^{2}-t_{1}+1}{t_{1}^{2}} \\
& & x^{4}: \frac{3}{2} t_{1}^{2}-2 t_{1}+\frac{3}{2} & y z^{2}: 0 & \\
Q_{2,0} & x z^{2} & x^{2} y^{3}: \frac{1}{\left(1-t_{1}\right)^{2}} & x^{3} y: \frac{t_{1}+1}{2\left(1-t_{1}\right)^{2}} & x y^{5}: \frac{t_{1}+1}{2 t_{1}\left(1-t_{1}\right)^{2}} \\
& & y^{7}: \frac{t_{1}^{2}-t_{1}+1}{t_{1}^{2}\left(1-t_{1}\right)^{2}} & y^{2} z^{2}: 0 &
\end{array}
$$

Thus $p_{>1}$ could be replaced in the normal form in table 10.1 by any of the following monomials:

$$
\begin{array}{llllll}
W_{1,0} & S_{1,0} & U_{1,0} & E_{3,0} & Z_{1,0} & Q_{2,0} \\
- & x^{2} y z, y^{4} z, y^{2} z^{2} & x^{2} y z, x y z^{2}, x y^{4} & - & - & x^{2} y^{3}
\end{array}
$$

We denote by $G_{3}$ and $G_{2} \subset G_{3}$ the groups of automorphisms of $T^{(2)}=\mathbb{P}^{1} \mathbb{C}$

$$
\begin{array}{ll}
G_{3}:=\left\{t_{1} \mapsto t_{1}, 1-t_{1}, \frac{1}{t_{1}}, \frac{t_{1}}{t_{1}-1}, \frac{1}{1-t_{1}}, \frac{t_{1}-1}{t_{1}}\right\} & \cong S_{3} \text { as a group, } \\
G_{2}:=\left\{t_{1} \mapsto t_{1}, 1-t_{1}\right\} \subset G_{3} & \cong S_{2} \text { as a group. }
\end{array}
$$


They act also on $T^{(1)}=\mathbb{C}-\{0,1\}$.

Theorem 10.3. Consider a bimodal family of quadrangle surface singularities in table (10.1). A function

$$
\begin{aligned}
& \kappa: G_{2} \times T^{(1)} \rightarrow \mathbb{C}^{*} \quad \text { for } W_{1,0}, S_{1,0}, \\
& \kappa: G_{3} \times T^{(1)} \rightarrow \mathbb{C}^{*}
\end{aligned}
$$

with the following properties exists.

$$
\begin{aligned}
f_{\left(t_{1}, t_{2}\right)} \sim_{\mathcal{R}} f_{\left(\widetilde{t}_{1}, \widetilde{t}_{2}\right)} & \Longleftrightarrow \quad \exists g \in \begin{cases}G_{2} & \text { for } W_{1,0}, S_{1,0}, \\
G_{3} & \text { for } U_{1,0}, E_{3,0}, Z_{1,0}, Q_{2,0},\end{cases} \\
& \text { with } \widetilde{t}_{1}=g\left(t_{1}\right), \widetilde{t}_{2}^{m_{\infty}}=\kappa\left(g, t_{1}\right) \cdot t_{2}^{m_{\infty}},(10.12) \\
\kappa\left(\mathrm{id}, t_{1}\right)= & 1, \\
\kappa\left(g_{2} g_{1}, t_{1}\right)= & \kappa\left(g_{1}, t_{1}\right) \cdot \kappa\left(g_{2}, g_{1}\left(t_{1}\right)\right) .
\end{aligned}
$$

Table 10.15 lists $\kappa\left(g, t_{1}\right)$ for generators $g$ of the group.

$$
\begin{array}{lllllll} 
& W_{1,0} & S_{1,0} & U_{1,0} & E_{3,0} & Z_{1,0} & Q_{2,0} \\
t_{1} \mapsto 1-t_{1} & 1 & -1 & 1 & \left(\frac{1-t_{1}}{t_{1}}\right)^{18} & \left(\frac{1-t_{1}}{t_{1}}\right)^{14} & -1 \\
t_{1} \mapsto t_{1}^{-1} & - & - & -t_{1}^{-3} & t_{1}^{-12} & t_{1}^{-10} & t_{1}^{3}
\end{array}
$$

Proof: 10.13 - 10.15 are consistent (to check this is nontrivial only for $E_{3,0}$ and $\left.Z_{1,0}\right)$ and define a unique function $\kappa$ as in 10.11. We will show now that it satisfies $\Leftarrow$ in $(10.12$. We postpone the proof of $\Rightarrow$ in 10.12 to the end of this section.

The equality

$$
f_{\left(t_{1}, t_{2}\right)}\left(x \cdot e^{2 \pi i w_{x}}, y \cdot e^{2 \pi i w_{y}}, z \cdot e^{2 \pi i w_{z}}\right)=f_{\left(t_{1}, t_{2} \cdot e^{2 \pi i 2 / m}\right)}
$$

gives $\Leftarrow$ in 10.12 for $g=\mathrm{id}$ and $\kappa\left(\mathrm{id}, t_{1}\right)=1$ (for $U_{1,0} m=m_{\infty}=9$, in the other cases $\left.m_{\infty}=\frac{m}{2}\right)$. We list now coordinate changes $(x, y, z) \mapsto$ $\varphi^{(1)}(x, y, z)$ and $(x, y, z) \mapsto \varphi^{(2)}(x, y, z)$ with

$$
\begin{aligned}
f_{\left(t_{1}, t_{2}\right)}\left(\varphi^{(1)}(x, y, z)\right)= & f_{\left(1-t_{1}, 0\right)}+t_{2} \cdot p^{(1)}\left(t_{1}, x, y, z\right) \\
& \text { for all } 6 \text { cases } \\
f_{\left(t_{1}, t_{2}\right)}\left(\varphi^{(2)}(x, y, z)\right)= & f_{\left(t_{1}^{-1}, 0\right)}(x, y, z)+t_{2} \cdot p^{(2)}\left(t_{1}, x, y, z\right) \\
& \text { for } U_{1,0}, E_{3,0}, Z_{1,0}, Q_{2,0}
\end{aligned}
$$


for certain quasihomogeneous polynomials $p^{(1)}$ and $p^{(2)}$ in $x, y, z$ with $\operatorname{deg}_{w} p^{(1)}=\operatorname{deg}_{w} p^{(2)}=1+\frac{2}{m}$.

$$
\begin{array}{lll} 
& \varphi^{(1)}(x, y, z) & \varphi^{(2)}(x, y, z) \\
W_{1,0} & (x,-y, z) & - \\
S_{1,0} & \left(i x, y,-z-y^{2}\right) & - \\
U_{1,0} & (-x+z,-y, z) & \left(-z, t_{1}^{-1 / 3} y,-x\right) \\
E_{3,0} & \left(x-y^{3},-y, z\right) & \left(x, t_{1}^{-1 / 3} y, z\right) \\
Z_{1,0} & \left(e^{-2 \pi i / 14}\left(x-y^{2}\right), i \cdot e^{-2 \pi i / 28} y, z\right) & \left(t_{1}^{1 / 7} x, t_{1}^{-3 / 7} y, z\right) \\
Q_{2,0} & \left(x-y^{2}, i y, e^{-2 \pi i / 8} z\right) & \left(x, t_{1}^{-1 / 2} y, t_{1}^{1 / 4} z\right)
\end{array}
$$

One can calculate $p^{(1)}$ and $p^{(2)}$ easily. The proof of AGV85, 12.6 Lemma] implies here

$$
\begin{aligned}
& f_{\left(\widetilde{t}_{1}, 0\right)}+t_{2} \cdot \widetilde{p} \sim_{\mathcal{R}} f_{\left(\widetilde{t}_{1}, \widetilde{t}_{2}\right)} \\
& \text { where } t_{2} \cdot \widetilde{p} \equiv \widetilde{t}_{2} \cdot p_{>1} \quad \bmod \left(\text { Jacobi ideal of } f_{\left(\widetilde{t}_{1}, 0\right)}\right) .
\end{aligned}
$$

With table 10.8 one finds $\widetilde{t}_{2}$ with 10.20 for $\widetilde{p}=p^{(1)}$ and for $\widetilde{p}=p^{(2)}$. Then one verifies table 10.15.

Remarks 10.4. (i) For the quasihomogeneous singularities, (10.12 becomes

$$
f_{\left(t_{1}, 0\right)} \sim_{\mathcal{R}} f_{\left(\widetilde{t}_{1}, 0\right)} \Longleftrightarrow \exists g \in G_{2} \text { resp. } G_{3} \text { with } \widetilde{t}_{1}=g\left(t_{1}\right) .
$$

This is proved in [Bi92, Satz 1.5.2] using the minimal good resolution. Our proof of $\Rightarrow$ in 10.12 for all singularities at the end of this section will be different.

(ii) The right equivalence classes in $T^{(5)}$ are the orbits of a group action on $T^{(5)}$ in the cases $W_{1,0}$ and $S_{1,0}$. There the group is a central extension of $G_{2}$ by a cyclic group of order $m_{\infty}=\frac{m}{2}$,

$$
1 \rightarrow\left(\begin{array}{l}
\text { cyclic group } \\
\text { of order } m
\end{array}\right) \rightarrow\left(\text { group acting on } T^{(5)}\right) \rightarrow G_{2} \rightarrow 1 .
$$

In the other cases $U_{1,0}, E_{3,0}, Z_{1,0}$ and $Q_{2,0}$, an $m$-th root of $\kappa\left(t_{1} \rightarrow\right.$ $\left.t_{1}^{-1},.\right): T^{(1)} \rightarrow \mathbb{C}^{*}$ is not uni-valued, but multi-valued. There one has only a groupoid acting on $T^{(5)}$, whose orbits are the right equivalence classes in $T^{(5)}$.

(iii) In any case, the space $M_{\mu}^{\text {mar }}=\left(M_{\mu}^{\text {mar }}\right)^{0} \cong T^{(7)}$ (by theorem 10.1 will be more canonical than $T^{(5)}$, and there the right equivalence classes are the orbits of the action of the group $G_{\mathbb{Z}}=G^{\text {mar }}$.

Now we come to the spectral numbers and the classifying space $D_{B L}$. 
Lemma 10.5. Consider a bimodal family of quadrangle surface singularities in table (10.1). Denote $\omega_{0}:=d x d y d z$.

(a) The spectral numbers $\alpha_{1}, \ldots, \alpha_{\mu}$ with $\alpha_{1} \leq \ldots \leq \alpha_{\mu}$ satisfy

$$
\begin{array}{r}
\alpha_{1}=\frac{-1}{m}<\alpha_{2}=\frac{1}{m}<\alpha_{3} \leq \ldots \leq \alpha_{\mu-2} \\
<\alpha_{\mu-1}=1-\frac{1}{m}<\alpha_{\mu}=1+\frac{1}{m} \\
\operatorname{dim} C^{\alpha_{1}}=\operatorname{dim} C^{\alpha_{2}}=2 .
\end{array}
$$

The following picture illustrates this.

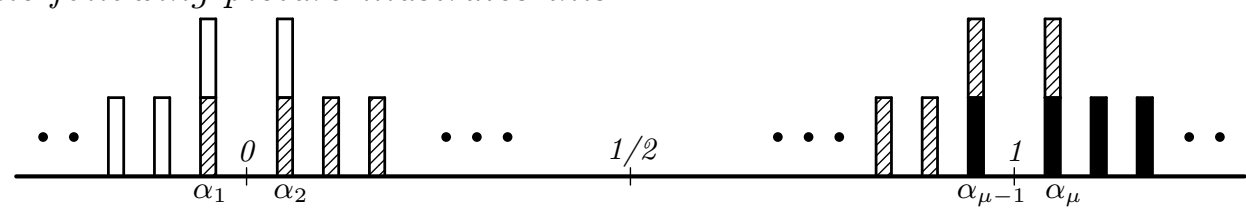

We also have

$$
\begin{aligned}
V^{\alpha_{1}}\left(f_{\left(t_{1}, t_{2}\right)}\right) & \supset H_{0}^{\prime \prime}\left(f_{\left(t_{1}, t_{2}\right)} \supset V^{>\alpha_{2}}\left(f_{\left(t_{1}, t_{2}\right)}\right),\right. \\
H_{0}^{\prime \prime}\left(f_{\left(t_{1}, t_{2}\right)}\right) & =\mathbb{C} \cdot\left(s\left(\omega_{0}, \alpha_{1}\right)\left(t_{1}, t_{2}\right)+s\left(\omega_{0}, \alpha_{2}\right)\left(t_{1}, t_{2}\right)\right) \\
& +\mathbb{C} \cdot s\left(y \omega_{0}, \alpha_{2}\right)\left(t_{1}, t_{2}\right)+V^{>\alpha_{2}}\left(f_{\left(t_{1}, t_{2}\right)}\right) .
\end{aligned}
$$

(b) The polarizing form $S$ defines an indefinite form $((a, b) \mapsto$ $S(a, \bar{b}))$ on $H_{\zeta}^{\infty}$. We get a half-plane

$$
\begin{aligned}
\mathcal{H}\left(C^{\alpha_{1}}\right) & :=\left\{\mathbb{C} \cdot v \mid v \in C^{\alpha_{1}} \text { with } S\left(\psi_{\alpha_{1}}^{-1}(v), \overline{\psi_{\alpha_{1}}^{-1}(v)}\right)<0\right\}(10.25) \\
& \subset \mathbb{P}^{1}\left(C^{\alpha_{1}}\right) .
\end{aligned}
$$

$$
\begin{aligned}
D_{B L} & =\left\{\mathbb{C} \cdot\left(v_{1}+v_{2}\right) \mid v_{1} \in C^{\alpha_{1}}-\{0\} \text { with }\left[\mathbb{C} \cdot v_{1}\right] \in \mathcal{H}\left(C^{\alpha_{1}}\right),\right. \\
& \left.\cong v_{2} \in \mathbb{C} \cdot \psi_{\alpha_{2}}\left(\overline{\psi_{\alpha_{1}}^{-1}\left(v_{1}\right)}\right) \subset C^{\alpha_{2}}\right\} \\
& \cong \mathcal{H}\left(C^{\alpha_{1}}\right) \times \mathbb{C} .
\end{aligned}
$$

Proof: (a) The spectral numbers are well known AGV88, 13.3.4, p. 389] and can be calculated in the semiquasihomogeneous cases for example with the generating series (here $m=2,\left(w_{0}, w_{1}, w_{2}\right)=$ $\left.\left(w_{x}, w_{y}, w_{z}\right)\right)$

$$
\prod_{j=0}^{m} \frac{t-t^{w_{j}}}{t^{w_{j}}-1}=\sum_{i=1}^{\mu} t^{\alpha_{i}+1} .
$$

10.22 and 10.23 are obvious. 10.24 follows from lemma 7.20 and $\operatorname{deg}_{w}\left(\omega_{0}\right)=\alpha_{1}+1, \operatorname{deg}_{w}\left(y \omega_{0}\right)=\alpha_{2}+1$ and $\operatorname{deg}_{w}\left(x^{i} y^{j} z^{k} \omega_{0}\right)>\alpha_{2}+1$ for any other monomial $x^{i} y^{j} z^{k}$, because $w_{y}<w_{x} \leq w_{z}$. 
(b) This follows as in section 9 before theorem 9.2. It follows also from the fact that $\operatorname{Gr}_{V}^{\bullet} H_{0}^{\prime \prime}\left(f_{\left(t_{1}, t_{2}\right)}\right)$ and $S$ induce as in (7.27) a polarized Hodge structure of weight 2 on $H^{\infty}\left(f_{\left(t_{1}, t_{2}\right)}\right)$. Especially,

$$
\begin{aligned}
& a_{1}\left(t_{1}, t_{2}\right):=\psi_{\alpha_{1}}^{-1} s\left(\omega_{0}, \alpha_{1}\right)\left(t_{1}, t_{2}\right) \in H^{\infty}\left(f_{\left(t_{1}, t_{2}\right)}\right)_{\zeta}, \\
& a_{2}\left(t_{1}, t_{2}\right):=\psi_{\alpha_{2}}^{-1} s\left(y \omega_{0}, \alpha_{2}\right)\left(t_{1}, t_{2}\right) \in H^{\infty}\left(f_{\left(t_{1}, t_{2}\right)}\right)_{\bar{\zeta}}
\end{aligned}
$$

satisfy

$$
\begin{array}{cl}
\text { on } H^{\infty}\left(f_{\left(t_{1}, t_{2}\right)}\right)_{\zeta}: & \mathbb{C} \cdot a_{1}=H^{2,0}=F^{2} \subset H^{\infty}\left(f_{\left(t_{1}, t_{2}\right)}\right)_{\zeta} \\
& =F^{1}=H^{2,0} \oplus H^{1,1}=\mathbb{C} \cdot a_{1} \oplus \mathbb{C} \cdot \overline{a_{2}}, \\
\text { on } H^{\infty}\left(f_{\left(t_{1}, t_{2}\right)}\right)_{\bar{\zeta}}: & \mathbb{C} \cdot a_{2}=H^{1,1}=F^{1} \subset H^{\infty}\left(f_{\left(t_{1}, t_{2}\right)}\right)_{\bar{\zeta}} \\
& =F^{0}=H^{1,1} \oplus H^{0,2}=\mathbb{C} \cdot a_{2} \oplus \mathbb{C} \cdot \overline{a_{1}}, \\
0<i^{2-0} S\left(a_{1}, \overline{a_{1}}\right), & 0<i^{1-1} S\left(\overline{a_{2}}, a_{2}\right), 0=S\left(a_{1}, a_{2}\right) .
\end{array}
$$

(c) This follows as in lemma 9.4 (c) in the case $m \mid p$.

The multi-valued period map $B L_{T^{(5)}}: T^{(5)} \rightarrow D_{B L}$ had been calculated in [He93]. We recall the result and sketch the proof. In part (e) of theorem 10.6 we add a formula for the case $S_{1,0}$ which will be useful for the determination of a transversal monodromy in theorem 10.7 .

Theorem 10.6. Consider a bimodal family of quadrangle surface singularities in table (10.1).

(a) $s\left(\omega_{0}, \alpha_{1}\right)\left(t_{1}, t_{2}\right)=s\left(\omega_{0}, \alpha_{1}\right)\left(t_{1}, 0\right)=s\left[\omega_{0}\right]\left(t_{1}, 0\right)$ is independent of $t_{2}$ and satisfies the hypergeometric differential equation

$$
0=\left(t_{1}\left(1-t_{1}\right) \partial_{t_{1}}^{2}+\left(c-(a+b+1) t_{1}\right) \partial_{t_{1}}-a b\right) s\left[\omega_{0}\right]\left(t_{1}, 0\right)
$$

with $(1-c, c-a-b, a-b)=\left(\frac{1}{m_{0}}, \frac{1}{m_{1}}, \frac{1}{m_{\infty}}\right)$.

(b) The multi-valued period map

$$
B L_{T^{(1)}}: T^{(1)} \rightarrow \mathcal{H}\left(C^{\alpha_{1}}\right), \quad t_{1} \mapsto \mathbb{C} \cdot s\left[\omega_{0}\right]\left(t_{1}, 0\right),
$$

lifts to a uni-valued period map

$$
B L_{T^{(3)}}: T^{(3)} \rightarrow \mathcal{H}\left(C^{\alpha_{1}}\right)
$$

which is an open embedding and extends to an isomorphism

$$
B L_{T^{(4)}}: T^{(4)} \rightarrow \mathcal{H}\left(C^{\alpha_{1}}\right) .
$$

(c)

$$
\begin{array}{r}
s\left(\omega_{0}, \alpha_{2}\right)\left(t_{1}, t_{2}\right)=t_{2} \cdot\left(-\partial_{\tau}\right) s\left[p_{>1} \omega_{0}\right]\left(t_{1}, 0\right), \\
C^{\alpha_{2}}=\mathbb{C} \cdot s\left[y \omega_{0}\right]\left(t_{1}, 0\right) \oplus \mathbb{C} \cdot \partial_{\tau} s\left[p_{>1} \omega_{0}\right]\left(t_{1}, 0\right) .
\end{array}
$$


(d) The multi-valued period map

$$
B L_{T^{(5)}}: T^{(5)} \rightarrow D_{B L}
$$

is locally in $T^{(1)}$ and $\mathcal{H}\left(C^{\alpha_{1}}\right)$ an isomorphism of line bundles and lifts to an open embedding of line bundles

$$
B L_{T^{(7)}}: T^{(7)} \rightarrow D_{B L}
$$

(We do not know whether this extends to an isomorphism of line bundles $T^{(8)} \rightarrow D_{B L}$, but we do not expect it.)

(e) In the case of $S_{1,0}$

$$
\partial_{t_{1}} s\left[x \omega_{0}\right]\left(t_{1}, 0\right)=\frac{2 t_{1}-1}{5 t_{1}\left(1-t_{1}\right)} \cdot s\left[x \omega_{0}\right]\left(t_{1}, 0\right) .
$$

Proof: (a) We just sketch the ansatz for the calculations which prove 10.32). $f_{\left(t_{1}, 0\right)}$ and $\partial_{t_{1}} f_{\left(t_{1}, 0\right)}$ are quasihomogeneous of weighted degree 1. List all monomials $d_{1}, \ldots, d_{l}$ in $x, y, z$ which turn up in $f_{\left(t_{1}, 0\right)}^{2}, f_{\left(t_{1}, 0\right)}$. $\partial_{t_{1}} f_{\left(t_{1}, 0\right)}$ and $\left(\partial_{t_{1}} f_{\left(t_{1}, 0\right)}\right)^{2}$, find $l-2$ independent linear combinations of $d_{1} \omega_{0}, \ldots, d_{l} \omega_{0}$ in $d f_{\left(t_{1}, 0\right)} \wedge d \Omega_{\mathbb{C}^{3}}^{1}$, and determine an equation

$$
\begin{aligned}
& p_{1} \cdot\left(\partial_{t_{1}} f_{\left(t_{1}, 0\right)}\right)^{2} \cdot \omega_{0}+p_{2} \cdot f_{\left(t_{1}, 0\right)} \cdot \partial_{t_{1}} f_{\left(t_{1}, 0\right)} \cdot \omega_{0}+p_{3} \cdot f_{\left(t_{1}, 0\right)}^{2} \cdot \omega_{0} \\
& \equiv 0 \quad \bmod d f_{\left(t_{1}, 0\right)} \wedge d \Omega_{\mathbb{C}^{3}}^{1}
\end{aligned}
$$

with $p_{1}, p_{2}, p_{3} \in \mathbb{Q}\left[t_{1}\right]$. Then

$$
\left(p_{1} \partial_{t_{1}}^{2}-\left(\alpha_{1}+2\right) p_{2} \partial_{t_{1}}+\left(\alpha_{1}+2\right)\left(\alpha_{1}+1\right) p_{3}\right) s\left[\omega_{0}\right]\left(t_{1}, 0\right) .
$$

Because of corollary 8.14 one can work in the cases $W_{1,0}, E_{3,0}, Z_{1,0}$ with the curve singularities. There the number $l$ of monomials is $l=5$. In the other cases, the surfaces singularities $S_{1,0}, U_{1,0}, Q_{2,0}$, it is $l=9$.

(b) The period map $B L_{T^{(1)}}$ is not constant because $s\left[\omega_{0}\right]\left(t_{1}, 0\right)$ and $\partial_{t_{1}} s\left[\omega_{0}\right]\left(t_{1}, 0\right)=\left(-\partial_{\tau}\right) s\left[\partial_{t_{1}} f_{\left(t_{1}, 0\right)} \cdot \omega_{0}\right]\left(t_{1}, 0\right)$ are linearly independent because $\partial_{t_{1}} f_{\left(t_{1}, 0\right)}$ is not in the Jacobi ideal. Therefore the multi-valued coefficient functions $f_{1}\left(t_{1}\right)$ and $f_{2}\left(t_{1}\right)$ with

$$
s\left[\omega_{0}\right]\left(t_{1}, 0\right)=f_{1}\left(t_{1}\right) \cdot v_{1}^{0}+f_{2}\left(t_{1}\right) \cdot v_{2}^{0}
$$

for an arbitrary basis $v_{1}^{0}, v_{2}^{0}$ of $C^{\alpha_{1}}$ are linearly independent scalar solutions of the same hypergeometric differential equation. Their quotient $\left(t_{1} \mapsto \frac{f_{1}\left(t_{1}\right)}{f_{2}\left(t_{1}\right)}\right)$ is a Schwarzian function [Fo51, sec. 113+114], which maps the closure of the upper half-plane to a hyperbolic triangle with angles $\frac{\pi}{m_{0}}, \frac{\pi}{m_{1}}, \frac{\pi}{m_{\infty}}$. The vertices are the images of $0,1, \infty$. Therefore the multi-valued map $B L_{T^{(1)}}: T^{(1)} \rightarrow \mathcal{H}\left(C^{\alpha_{1}}\right)$ is an inverse of the quotient map $c^{(1)}: T^{(3)} \rightarrow T^{(1)}$. This shows 10.34 and 10.35. 
(c) $s\left(\omega_{0}, \alpha_{2}\right)\left(t_{1}, 0\right)=0$ because of formula 7.52 in lemma 7.20 (a).

$$
\begin{aligned}
\partial_{t_{2}} s\left(\omega_{0}, \alpha_{2}\right)\left(t_{1}, t_{2}\right) & =\left(-\partial_{\tau}\right) s\left(p_{>1} \omega_{0}, \alpha_{2}+1\right)\left(t_{1}, t_{2}\right) \\
& =\left(-\partial_{\tau}\right) s\left[p_{>1} \omega_{0}\right]\left(t_{1}, 0\right) \\
\text { thus } s\left(\omega_{0}, \alpha_{2}\right)\left(t_{1}, t_{2}\right) & =t_{2} \cdot\left(-\partial_{\tau}\right) s\left[p_{>1} \omega_{0}\right]\left(t_{1}, 0\right) \\
& \equiv t_{2} \cdot v_{2} \bmod \mathbb{C} \cdot s\left[y \omega_{0}\right]\left(t_{1}, 0\right) \\
\text { with a suitable } v_{2} & \in \psi_{\alpha_{2}}^{-1}\left(\overline{\psi_{\alpha_{1}}\left(s\left[\omega_{0}\right]\left(t_{1}, 0\right)\right)}\right)-\{0\} .
\end{aligned}
$$

Here $v_{2} \neq 0$ follows from (10.37) which is a consequence of the fact that $p_{>1}$ is not in the Jacobi ideal of $f_{\left(t_{1}, 0\right)}$.

(d) This follows from 10.34 and part (c).

(e) The proof is similar to the calculations which prove part (a), but simpler.

$$
\begin{aligned}
& \partial_{t_{1}} s\left[x \omega_{0}\right]\left(t_{1}, 0\right) \\
= & \left(-\partial_{\tau}\right) s\left[\partial_{t_{1}} f_{\left(t_{1}, 0\right)} \cdot x \omega_{0}\right]\left(t_{1}, 0\right)=\left(-\partial_{\tau}\right) s\left[x^{3} y^{2} \omega_{0}\right]\left(t_{1}, 0\right) \\
\stackrel{(*)}{=} & \frac{2 t_{1}-1}{6 t_{1}\left(t_{1}-1\right)}\left(-\partial_{\tau}\right) s\left[f_{\left(t_{1}, 0\right)} \cdot x \omega_{0}\right]\left(t_{1}, 0\right) \\
= & \frac{2 t_{1}-1}{6 t_{1}\left(t_{1}-1\right)}\left(-\partial_{\tau} \tau\right) s\left[x \omega_{0}\right]\left(t_{1}, 0\right)=\frac{2 t_{1}-1}{6 t_{1}\left(t_{1}-1\right)}\left(-\frac{6}{5}\right) s\left[x \omega_{0}\right]\left(t_{1}, 0\right) \\
= & \frac{2 t_{1}-1}{5 t_{1}\left(1-t_{1}\right)} s\left[x \omega_{0}\right]\left(t_{1}, 0\right) .
\end{aligned}
$$

For $\stackrel{(*)}{=}$ one has to find 3 relations in $d f_{\left(t_{1}, 0\right)} \wedge d \Omega_{\mathbb{C}^{3}}^{1}$ between the monomial differential forms $x^{3} y^{2} \omega_{0}, x y^{3} z \omega_{0}, x y z^{2} \omega_{0}$ and $x^{3} z \omega_{0}$ in $f_{\left(t_{1}, 0\right)} \cdot x \omega_{0}$ and $x^{3} y^{2} \omega_{0}$.

The last step before the proof of theorem 10.1 is the following result on a transversal monodromy group. Its proof uses formula (6.8) in theorem 6.1 .

Theorem 10.7. Consider a bimodal family of quadrangle surface singularities in table $(10.1)$. The pull back to $T^{(3)}$ with $c^{(1)}$ of the homology group $\bigcup_{t_{1} \in T^{(1)}} M l\left(f_{\left(t_{1}, 0\right)}\right) \rightarrow T^{(1)}$ comes equipped with a monodromy representation $\pi^{(3)}: \pi_{1}\left(T^{(3)}, \tau^{(3)}\right) \rightarrow G_{\mathbb{Z}}$ (with $c^{(1)}\left(\tau^{(3)}\right)=i$ ) which is called transversal monodromy group.

(a) The following table lists the local monodromies around elliptic fixed points in $\left(c^{(2)}\right)^{-1}(0),\left(c^{(2)}\right)^{-1}(1)$ and $\left(c^{(2)}\right)^{-1}(\infty)$.

$$
\begin{array}{lllllll} 
& W_{1,0} & S_{1,0} & U_{1,0} & E_{3,0} & Z_{1,0} & Q_{2,0} \\
\left(c^{(2)}\right)^{-1}(\{0,1\}) & \text { id } & \text { id } & \text { id } & \text { id } & \text { id } & \text { id } \\
\left(c^{(2)}\right)^{-1}(\infty) & \text { id } & M_{h}^{5} & \text { id } & \text { id } & \text { id } & M_{h}^{6}
\end{array}
$$


Therefore $\operatorname{Im}\left(\pi^{(3)}\right)=\{\mathrm{id}\}$ for $W_{1,0}, U_{1,0}, E_{3,0}, Z_{1,0}$, and $\operatorname{Im}\left(\pi^{(3)}\right)=$ $\left\{\mathrm{id}, M_{h}^{m_{\infty}}\right\}$ for $S_{1,0}$ and $Q_{2,0}$.

(b)

$$
\begin{aligned}
& \left\{g \in G_{\mathbb{Z}} \mid g \text { acts trivially on } D_{B L}\right\} \\
= & \left\{g \in G_{\mathbb{Z}} \mid g= \pm \mathrm{id} \text { on } M l_{\zeta}\right\} \\
= & \left\{ \pm \mathrm{id}, \pm M_{h}^{m_{\infty}}\right\} \\
= & \begin{cases}\{ \pm \mathrm{id}\} & \text { for } U_{1,0}, E_{3,0}, Z_{1,0} \\
\left\{ \pm \mathrm{id}, \pm M_{h}^{m_{\infty}}\right\} & \text { for } W_{1,0}, S_{1,0}, Q_{2,0} .\end{cases}
\end{aligned}
$$

(c) $G_{\mathcal{R}}^{\text {smar,gen }}$ is here the group in 8.13 for the singularities of multiplicity $\geq 3$, namely the curve singularities $W_{1,0}, E_{3,0}, Z_{1,0}$ and the surface singularities $S_{1,0}, U_{1,0}, Q_{2,0}$.

$$
G_{\mathcal{R}}^{\text {smar,gen }}= \begin{cases}\{\mathrm{id}\} & \text { for } U_{1,0}, E_{3,0}, Z_{1,0} \\ \left\{\mathrm{id}, M_{h}^{m_{\infty}}\right\} & \text { for } W_{1,0}, S_{1,0}, Q_{2,0}\end{cases}
$$

Proof: We start with part (b). Suppose that $g \in G_{\mathbb{Z}}$ acts trivially on $D_{B L}$. Then it acts trivially on $\mathcal{H}\left(C^{\alpha_{1}}\right)$, so $g=\lambda$. id on $M l_{\zeta}$ for some $\lambda \in \mathbb{C}^{*}$. And $\mathbb{C} \cdot\left(v_{1}+v_{2}\right)=\mathbb{C} \cdot\left(\lambda v_{1}+\bar{\lambda} v_{2}\right)$, so $\lambda=\bar{\lambda} \in\{ \pm 1\}$. This together with formula 6.8 and the set of eigenvalues of $M_{h}$ gives (10.46).

(a) The Papperitz-Riemann symbol

$$
\left\{\begin{array}{cccc}
0 & 1 & \infty \\
0 & 0 & a & z \\
1-c & c-a-b & b
\end{array}\right\}
$$

encodes the local behaviour near 0,1 and $\infty$ of scalar solutions of the hypergeometric equation. Locally suitable solutions have the following form (h.o.t. = higher order terms):

$$
\begin{aligned}
& \text { near } 0 \text { : } \quad t_{1}^{0}+\text { h.o.t. } \quad \text { and } t_{1}^{1-c}+\text { h.o.t., } \\
& \text { near } 1 \text { : } \quad\left(t_{1}-1\right)^{0}+\text { h.o.t. } \quad \text { and } \quad\left(t_{1}-1\right)^{c-a-b}+\text { h.o.t., } \\
& \text { near } \infty \text { : } t_{1}^{-a}+\text { h.o.t. } \quad \text { and } t_{1}^{-b}+\text { h.o.t. }
\end{aligned}
$$

Especially, the local monodromy of the space of solutions has the eigenvalues

$$
\begin{aligned}
& \text { around } 0: 1 \text { and } e^{2 \pi i(1-c)} \text {, } \\
& \text { around 1: } 1 \text { and } e^{2 \pi i(c-a-b)} \text {, } \\
& \text { around } \infty \text { : } e^{-2 \pi i a} \text { and } e^{-2 \pi i b} \text {. }
\end{aligned}
$$


In our situation $(1-c, c-a-b, a-b)=\left(\frac{1}{m_{0}}, \frac{1}{m_{1}}, \frac{1}{m_{\infty}}\right)$,

$$
\begin{array}{lllllll} 
& W_{1,0} & S_{1,0} & U_{1,0} & E_{3,0} & Z_{1,0} & Q_{2,0} \\
a & \frac{1}{2} & \frac{1}{2} & \frac{4}{9} & \frac{4}{9} & \frac{3}{7} & \frac{5}{12} \\
b & \frac{1}{3} & \frac{3}{10} & \frac{1}{3} & \frac{1}{3} & \frac{2}{7} & \frac{1}{4} \\
c & \frac{11}{12} & \frac{9}{10} & \frac{8}{9} & \frac{8}{9} & \frac{6}{7} & \frac{5}{6}
\end{array}
$$

The branched covering $c^{(2)}: T^{(4)} \rightarrow T^{(2)}$ has at elliptic fixed points the orders $m_{0}, m_{1}, m_{\infty}$. Therefore the local monodromies of the pull back to $T^{(3)}$ of the solutions on $T^{(1)}=\mathbb{C}-\{0,1\} \subset T^{(2)}=\mathbb{P}^{1} \mathbb{C}$ become + id except around the elliptic fixed points in $\left(c^{(2)}\right)^{-1}(\infty)$ in the cases $S_{1,0}$ and $Q_{2,0}$ where they become - id.

The same holds for the restrictions to $M l_{\zeta}$ of the local monodromies in $\pi^{(3)}$.

With 6.8 we obtain (10.45) for $U_{1,0}, E_{3,0}, Z_{1,0}$ and the following approximation of 10.45 for $W_{1,0}, S_{1,0}, Q_{2,0}$.

$$
\begin{array}{llll} 
& W_{1,0} & S_{1,0} & Q_{2,0} \\
\left(c^{(2)}\right)^{-1}(\{0,1\}) & \text { id or }-M_{h}^{6} & \text { id or }-M_{h}^{5} & \text { id or }-M_{h}^{6} \\
\left(c^{(2)}\right)^{-1}(\infty) & \text { id or }-M_{h}^{6} & - \text { id or } M_{h}^{5} & - \text { id or } M_{h}^{6}
\end{array}
$$

The case $W_{1,0}$ : The sublattice $M l_{-1, \mathbb{Z}}$ has rank 1 . Therefore the local transversal monodromies of the homology bundle on $T^{(1)}$ around 0,1 and $\infty$ have on $M l_{-1, \mathbb{Z}}$ eigenvalues in $\{ \pm 1\}$. The branched covering $T^{(4)} \rightarrow T^{(2)}$ is at the elliptic fixed points of even order. Thus $\pi^{(3)}$ restricts to the trivial monodromy on $M l_{-1, \mathbb{Z}}$. This excludes $-M_{h}^{6}$ in (10.52).

The case $S_{1,0}$ : The local transversal monodromies of the homology bundle on $T^{(1)}$ around 0,1 and $\infty$ have on $M l_{e^{-2 \pi i / 5}}$ eigenvalues in $\operatorname{Eiw}(\zeta)$. The branched covering is at the elliptic fixed points in $\left(c^{(2)}\right)^{-1}(\{0,1\})$ of order 10 . Thus the local monodromies of $\pi^{(3)}$ around points in $\left(c^{(2)}\right)^{-1}(\{0,1\})$ are trivial on $M l_{e^{-2 \pi i / 5}}$. This excludes $-M_{h}^{5}$ in the first line of $(10.52)$. The branched covering is at the elliptic fixed points in $\left(c^{(2)}\right)^{-1}(\infty)$ of order 5 . Formula 10.40 in theorem 10.6 gives near $\infty$

$$
s\left[x \omega_{0}\right]\left(t_{1}, 0\right)=\left(t_{1}^{-2 / 5}+\text { h.o.t. }\right) \cdot(\text { a flat multi-valued section }) .(10.53)
$$

Therefore also the local monodromy of $\pi^{(3)}$ around points in $\left(c^{(2)}\right)^{-1}(\infty)$ is trivial. This excludes - id in the second line of $(10.52)$.

The case $Q_{2,0}$ : The local transversal monodromies of the homology bundle on $T^{(1)}$ around 0,1 and $\infty$ have on $M l_{e^{-2 \pi i / 3}}$ eigenvalues in $\operatorname{Eiw}\left(e^{2 \pi i / 6}\right)$. The branched covering $T^{(4)} \rightarrow T^{(2)}$ is at the elliptic fixed points of order 6 . Thus $\pi^{(3)}$ restricts to the trivial monodromy on 
$M l_{e^{-2 \pi i / 3}}$. This excludes $-M_{h}^{6}$ in the first line and $-\mathrm{id}$ in the second line of 10.52 .

(c) - id $\notin G_{\mathcal{R}}^{\text {smar,gen }}$ by theorem $8.8(\mathrm{~d}) . G_{\mathcal{R}}^{\text {smar,gen }}$ fixes $B L(f, \pm \rho)$ for any $(f, \pm \rho) \in M_{\mu}^{\text {mar }}$. Because $T^{(7)} \rightarrow D_{B L}$ is an open embedding, $G_{\mathcal{R}}^{\text {smar,gen }}$ fixes $D_{B L}$. By part (b) $G_{\mathcal{R}}^{\text {smar,gen }}=\{$ id $\}$ for $U_{1,0}, E_{3,0}, Z_{1,0}$, and $G_{\mathcal{R}}^{\text {smar,gen }}=\{\mathrm{id}\}$ or $\left\{\mathrm{id}, M_{h}^{m_{\infty}}\right\}$ or $\left\{\mathrm{id},-M_{h}^{m_{\infty}}\right\}$ for $W_{1,0}, S_{1,0}, Q_{2,0}$. The coordinate changes $\varphi$ of the curve singularities $W_{1,0}$ and the surface singularities $S_{1,0}$ and $Q_{2,0}$ in the following table give a nontrivial element of $G_{\mathcal{R}}^{\text {smar,gen }}$.

$$
\begin{array}{lll}
W_{1,0} & S_{1,0} & Q_{2,0} \\
(x, y) \mapsto(-x, y) & (x, y, z) \mapsto(-x, y, z) & (x, y, z) \mapsto(x, y,-z)
\end{array}
$$

The coordinate change $\varphi$ maps $\omega_{0}$ to $-\omega_{0}$ and $s\left[\omega_{0}\right]\left(t_{1}, 0\right)$ to $-s\left[\omega_{0}\right]\left(t_{1}, 0\right)$. Therefore $\left.(\varphi)_{h o m}\right|_{M l_{\zeta}}=-\mathrm{id}$ and $(\varphi)_{h o m}=M_{h}^{m_{\infty}}$ (and not $\left.-M_{h}^{m_{\infty}}\right)$. This shows (10.46) for $W_{1,0}, S_{1,0}, Q_{2,0}$.

Finally we come to the proof of theorem 10.1. Within this proof, we will also finish the proof of theorem 6.1. After it, we will finish the proof of theorem 10.3 .

Proof of theorem 10.1: By theorem 10.7 (a)+(c), the transversal monodromy representation $\pi^{(7)}$ of the pull back to $T^{(7)}$ with $c^{(5)}$ of the homology bundle $\bigcup_{\left(t_{1}, t_{2}\right) \in T^{(5)}} M l\left(f_{\left(t_{1}, t_{2}\right)}\right) \rightarrow T^{(5)}$ is trivial in the cases $W_{1,0}, U_{1,0}, E_{3,0}, Z_{1,0}$ and has image in $G_{\mathcal{R}}^{\text {smar,gen }}=\left\{\mathrm{id}, M_{h}^{m_{\infty}}\right\}$ in the cases $S_{1,0}$ and $Q_{2,0}$. Thus the strong marking + id on $f_{(i, 0)}$ induces for each $f_{\left(t_{1}, t_{2}\right)}$ two strong markings in the same right equivalence class in the cases $S_{1,0}$ and $Q_{2,0}$ and one strong marking in the other cases. In any case, this gives a map $T^{(7)} \rightarrow\left(M_{\mu}^{\text {smar }}\right)^{0}$.

The composition $T^{(7)} \rightarrow\left(M_{\mu}^{\text {smar }}\right)^{0} \rightarrow D_{B L}$ is an open embedding by theorem 10.6. Also recall that $\left(M_{\mu}^{\text {smar }}\right)^{0} \rightarrow D_{B L}$ is an immersion and that all three spaces are 2-dimensional manifolds. Therefore $T^{(7)} \rightarrow$ $\left(M_{\mu}^{\text {smar }}\right)^{0}$ and $\left(M_{\mu}^{\text {smar }}\right)^{0} \rightarrow D_{B L}$ are open embeddings. We postpone the proof that the map $T^{(7)} \rightarrow\left(M_{\mu}^{\text {smar }}\right)^{0}$ is an isomorphism.

Part (b) follows now easily: Consider the case of singularities of multiplicity $\geq 3$. $-\mathrm{id} \in G_{\mathbb{Z}}$ acts trivially on $D_{B L}$. It acts nontrivially on $M_{\mu}^{\text {smar }}$ by theorem 8.5 (c). The map $\left(M_{\mu}^{\text {smar }}\right)^{0} \rightarrow D_{B L}$ is an embedding. Therefore $-\mathrm{id} \in G_{\mathbb{Z}}$ does not act on $\left(M_{\mu}^{\text {smar }}\right)^{0}$. Therefore - id $\notin G^{s m a r}$. This shows part (b). In this case $\left(M_{\mu}^{s m a r}\right)^{0} \cong\left(M_{\mu}^{\text {mar }}\right)^{0}$ by theorem 8.5 (c).

In the case of singularities of multiplicity $2, M_{\mu}^{s m a r}=M_{\mu}^{\text {mar }}$ and $\left(M_{\mu}^{\text {smar }}\right)^{0}=\left(M_{\mu}^{\text {mar }}\right)^{0}$ hold anyway. 
$c^{(2)}: T^{(4)}=\mathbb{H} \rightarrow T^{(2)}=\mathbb{P}^{1} \mathbb{C}$ is the branched covering from an action of a triangle group $\Gamma$ of type $\left(\frac{1}{m_{0}}, \frac{1}{m_{1}}, \frac{1}{m_{\infty}}\right)$ on $\mathbb{H}$. The group $\Gamma$ is a normal subgroup of index 2 respectively 6 of a triangle group $\Gamma^{q h}$ of type $(2,2 m, 2 m)$ for $W_{1,0}$ and $S_{1,0}$ and of type $(2,3,2 m)$ for $U_{1,0}, E_{3,0}, Z_{1,0}$ and $Q_{2,0}$ such that $\Gamma^{q h} / \Gamma=\left(G_{2}\right.$ respectively $\left.G_{3}\right)$. The following pictures show hyperbolic triangles associated to $\Gamma$ and $\Gamma^{q h}$. The symbols $[0],[1],[\infty],\left[\frac{1}{2}\right],[2],[-1],\left[e^{2 \pi i / 6}\right]$ at special points indicate the images of these points under $c^{(2)}$.
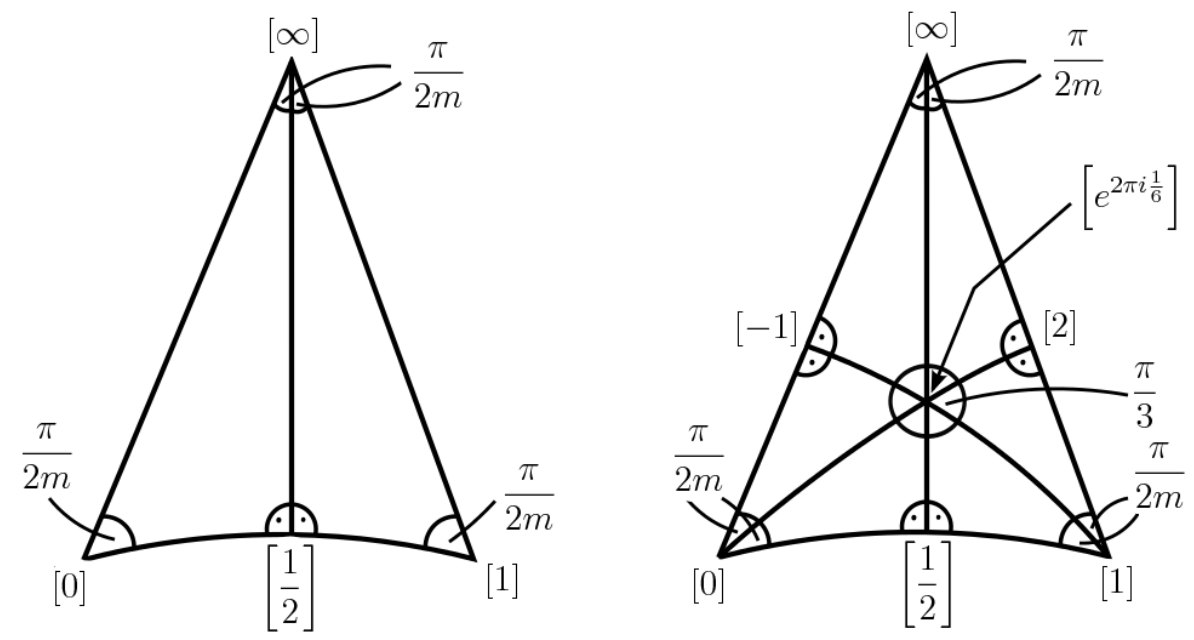

The group $\Gamma^{q h}$ maps the set of elliptic fixed points $\left(c^{(2)}\right)^{-1}(\{0,1, \infty\})=$ $T^{(4)}-T^{(3)}$ of $\Gamma$ to itself, so it acts on $T^{(3)}$.

By the proved implication $\Leftarrow$ in $\sqrt{10.12}$ in theorem 10.3 , the orbits of $\Gamma^{q h}$ in $T^{(3)}$ are contained in the right equivalence classes of quasihomogeneous singularities. By the embedding $T^{(3)} \rightarrow \mathcal{H}\left(C^{\alpha_{1}}\right)$ in theorem 10.6. $\Gamma^{q h}$ acts also on $\mathcal{H}\left(C^{\alpha_{1}}\right)$, and the orbits are contained in the orbits of $\Psi\left(G^{\text {mar }}\right)$, because the orbits of $G^{\text {mar }}$ on $\left(M_{\mu}^{\text {mar }}\right)^{0}$ are the right equivalence classes in $\left(M_{\mu}^{\text {mar }}\right)^{0}$.

Now compare the actions of $\Gamma^{q h}$ and $\Psi\left(G^{\text {mar }}\right)$ on $\mathcal{H}\left(C^{\alpha_{1}}\right) . \Gamma^{q h}$ acts as a triangle group of type $(2,2 m, 2 m)$ respectively $(2,3,2 m)$, and $\Psi\left(G^{\text {mar }}\right)$ acts by theorem 6.1 (b) as a subgroup of a triangle group of the same type. And the orbits of $\Gamma^{q h}$ are contained in the orbits of $\Psi\left(G^{\text {mar }}\right)$. Therefore the actions coincide, and $\Psi\left(G^{\text {mar }}\right)=\Psi\left(G_{\mathbb{Z}}\right)$ is a triangle group of the claimed type in 6.7$)$. This gives the surjectivity in theorem 6.1 and finishes the proof of theorem 6.1.

It also shows that $G^{\text {mar }}$ acts on $T^{(3)}$. Because $T^{(3)}$ contains representatives of the right equivalence classes of all quasihomogeneous 
singularities in the given $\mu$-homotopy family, the marked quasihomogeneous singularities in $\left(M_{\mu}^{\text {mar }}\right)^{0}$ must all be in $T^{(3)}$. This proves that the open embedding $T^{(7)} \rightarrow\left(M_{\mu}^{\text {mar }}\right)^{0}$ is an isomorphism.

Next we will prove $G_{\mathbb{Z}}=G^{\text {mar }}$. Consider an element $g_{1} \in G_{\mathbb{Z}}$. Because of $\Psi\left(G^{\text {mar }}\right)=\Psi\left(G_{\mathbb{Z}}\right)$, we can multiply it with an element $g_{2} \in G^{\text {mar }}$ such that $g_{3}=g_{1} g_{2}$ satisfies $\Psi\left(g_{3}\right)=$ id. By formula (6.8) in theorem 6.1 $g_{3} \in\left\{ \pm M_{h}^{k} \mid k \in \mathbb{Z}\right\} \subset G^{\text {mar }}$. This proves $G_{\mathbb{Z}}=G^{\text {mar }}$.

Now $M_{\mu}^{\text {mar }}=\left(M_{\mu}^{\text {mar }}\right)^{0}$ holds. Because $B L:\left(M_{\mu}^{\text {mar }}\right)^{0} \rightarrow D_{B L}$ is an embedding, $B L: M_{\mu}^{\text {mar }} \rightarrow D_{B L}$ is an embedding. This finishes the proof of theorem 10.1 .

Proof of $\Rightarrow$ in 10.12 in theorem 10.3: $G_{\mathbb{Z}}$ acts as $\Gamma^{q h}$ on $\mathcal{H}\left(C^{\alpha_{1}}\right)$ and thus as $G_{2}$ respectively $G_{3}$ on $T^{(1)}$. This shows $\Rightarrow$ in 10.12 for the quasihomogeneous singularities.

An element $g \in G_{\mathbb{Z}}$ which acts trivially on $T^{(3)}$ is in $\left\{ \pm M_{h}^{k} \mid k \in \mathbb{Z}\right\}$ and restricts to $\lambda \cdot$ id on $M l_{\zeta}$ for some $\lambda \in \operatorname{Eiw}(\zeta)$. Because of

$$
g: \mathbb{C} \cdot\left(v_{1}+v_{2}\right) \mapsto \mathbb{C}\left(\lambda \cdot v_{1}+\bar{\lambda} \cdot v_{2}\right)=\mathbb{C} \cdot\left(v_{1}+\bar{\lambda}^{2} \cdot v_{2}\right)
$$

it acts on the fibers of the projection $D_{B L} \rightarrow \mathcal{H}\left(C^{\alpha_{1}}\right)$ by multiplication with $\bar{\lambda}^{2}$, and it acts in the same way on the fibers of the projection $T^{(7)} \rightarrow T^{(3)}$. But $\left(\bar{\lambda}^{2}\right)^{m_{\infty}}=1$. This shows $\Rightarrow$ in 10.12 for all singularities.

\section{REFERENCES}

[AGV85] V.I. Arnold, S.M. Gusein-Zade, A.N. Varchenko: Singularities of differentiable maps, volume I. Birkhäuser, Boston, Basel, Stuttgart, 1985.

[AGV88] V.I. Arnold, S.M. Gusein-Zade, A.N. Varchenko: Singularities of differentiable maps, volume II. Birkhäuser, Boston 1988.

[BH17] S. Balnojan, C. Hertling: Real Seifert forms and polarizing forms of Steenbrink mixed Hodge structures. Manuscript, September 2017, 38 pages.

[Bi92] F.J. Bilitewski: Deformationen von Dreiecks- und Viereckssingularitäten der Einbettungsdimension drei. Doctoral thesis. Bonner Math. Schriften 237, Bonn 1992.

[BS66] Z.I. Borevich, I.R. Shafarevich: Number theory. Academic Press, New York and London, 1966.

[Br70] E. Brieskorn: Die Monodromie der isolierten Singularitäten von Hyperflächen. Manuscripta Math. 2 (1970), 103-161.

[Eb81] W. Ebeling: Quadratische Formen und Monodromiegruppen von Singularitäten. Math. Ann. 255 (1981), 463-498.

[Eb07] W. Ebeling: Functions of several complex variables and their singularities. Graduate Studies in Mathematics 83, AMS, 2007.

[Fo51] L.R. Ford: Automorphic functions. Chelsea Publishing Company, New York, 1951. 
[GH17] F. Gauss, C. Hertling: $\quad \mu$-constant monodromy groups and Torelli results for marked singularities, for the unimodal and some bimodal singularities. In: Singularities and Computer Algebra, Festschrift for Gert-Martin Greuel on the Occasion of his 70th Birthday (W. Decker, G. Pfister, M. Schulze, eds.). Springer International Publishing, 2017, 109-146.

[He93] C. Hertling: Analytische Invarianten bei den unimodularen und bimodularen Hyperflächensingularitäten. Doctoral thesis. Bonner Math. Schriften 250, Bonn 1993.

[He95] C. Hertling: Ein Torellisatz für die unimodalen und bimodularen Hyperflächensingularitäten. Math. Ann. 302 (1995), 359-394.

[He98] C. Hertling: Brieskorn lattices and Torelli type theorems for cubics in $\mathbb{P}^{3}$ and for Brieskorn-Pham singularities with coprime exponents. In: Singularities, the Brieskorn anniversary volume. Progress in Mathematics 162. Birkhäuser Verlag, Basel-Boston-Berlin 1998, 167-194.

[He99] C. Hertling: Classifying spaces and moduli spaces for polarized mixed Hodge structures and for Brieskorn lattices. Compositio Math. 116 (1999), $1-37$.

[He02] C. Hertling: Frobenius manifolds and moduli spaces for singularities. Cambridge Tracts in Mathematics 151, Cambridge University Press, 2002.

[He03] C. Hertling: $\quad t t^{*}$ geometry, Frobenius manifolds, their connections, and the construction for singularities. J. reine angew. Math 555 (2003), 77-161.

[He05] C. Hertling: Formes bilinéaires et hermitiennes pour des singularités: un aperçu. In: Singularités (ed. D. Barlet), Institut Élie Cartan Nancy 18, 2005, $1-17$.

[He11] C. Hertling: $\quad \mu$-constant monodromy groups and marked singularities. Ann. Inst. Fourier, Grenoble 61.7 (2011), 2643-2680.

[KV85] A.G. Khovanskii, A.N. Varchenko: Asymptotics of integrals over vanishing cycles and the Newton polyhedron. Sov. Math. Dokl. 32.1 (1985), 122-127.

[Ko76] A.G. Kouchnirenko: Polyèdres de Newton et nombres de Milnor. Invent. Math. 32 (1976), 1-31.

[Ku98] Va.S. Kulikov: Mixed Hodge structures and singularities. Cambridge tracts in mathematics 132, Cambridge University Press, 1998.

[LR73] Lê Dũng Tráng, C.P. Ramanujam: The invariance of Milnor's number implies the invariance of the topological type. Amer. J. of Math. 98 (1973), $67-78$.

[Le64] J. Lehner: Discontinuous groups and automorphic functions. Mathematical Surveys and Monographs 8, AMS, 1964.

[Ma68] J. Mather: Stability of $C^{\infty}$-maps III. Finitely determined map germs. Publ. Math. I.H.E.S. 35 (1968), 127-156.

[MW86] F. Michel, C. Weber: Sur le rôle de la monodromie entière dans la topologie des singularités. Ann. Inst. Fourier Grenoble 36 (1986), 183-218.

[Mi68] J. Milnor: Singular points of complex hypersurfaces. Ann. Math. Stud. vol. 61, Princeton University Press 1968.

[Or72] P. Orlik: On the homology of weighted homogeneous polynomials. In: Lecture Notes in Math. 298, Springer, Berlin, 1972.

[Ph85] F. Pham: La descente des cols par les onglets de Lefschetz, avec vues sur Gauss-Manin. In: Systémes differentiels et singularités. Asterisque 130 (1985), $11-47$. 
[SaK83] K. Saito: The higher residue pairings $K_{F}^{(k)}$ for a family of hypersurface singular points. In: Singularities. Proc. of symp. in pure math. 40.2 (1983), 441-463.

[SaM88] M. Saito: Exponents and Newton polyhedra of isolated hypersurface singularities. Math. Ann. 281 (1988), 411-417.

[SaM89] M. Saito: On the structure of Brieskorn lattices. Ann. Inst. Fourier Grenoble 39 (1989), 27-72.

[SaM91] M. Saito: Period mapping via Brieskorn modules. Bull. Soc. math. France 119 (1991), 141-171.

[SS85] J. Scherk, J.H.M. Steenbrink: On the mixed Hodge structure on the cohomology of the Milnor fibre. Math. Ann. 271 (1985), 641-665.

[Sch06] W.A. Schmid: On the set of integral solutions of the Pell equation in number fields. Aequationes Math. 71 (2006), 109-114.

[Sh71] G. Shimura: Introduction to the arithmetic theory of automorphic functions. Iwami Shoten, Publishers, and Princeton University Press, 1971.

[St77] J.H.M. Steenbrink: Mixed Hodge structure on the vanishing cohomology. In: Real and complex singularities, Oslo 1976, P. Holm (ed.). Alphen aan den Rijn: Sijthoff and Noordhoff 1977, 525-562.

[Ta75] K. Takeuchi: A characterization of arithmetic Fuchsian groups. Journal Math. Soc. Japan 27.4 (1975), 600-612.

[Ta77] K. Takeuchi: $\quad$ Arithmetic triangle groups. Journal Math. Soc. Japan 29.1 (1977), 91-106.

[Va80-1] A.N. Varchenko: The asymptotics of holomorphic forms determine a mixed Hodge structure. Sov. Math. Dokl. 22 (1980), 772-775.

[Va80-2] A.N. Varchenko: The Gauss-Manin connection of isolated singular point and Bernstein polynomial. Bull. des sciences math. 2e ser. 104 (1980), 205-223.

[Va82] A.N. Varchenko: The complex singular index does not change along the stratum $\mu=$ constant. Functional Anal. Appl. 16 (1982), 1-9.

[Wa97] L.C. Washington: Introduction to cyclotomic fields. Second edition. Graduate texts in mathematics 83, Springer, 1997.

Falko Gauss, Universität Mannheim, Lehrstuhl für Mathematik VI, Seminargebäude A 5, 6, 68131 Mannheim, Germany

E-mail address: gauss@math.uni-mannheim.de

Claus Hertling, Universität Mannheim, Lehrstuhl für Mathematik Vi, Seminargebäude A 5, 6, 68131 Mannheim, Germany

E-mail address: hertling@math.uni-mannheim.de 